\title{
Towards Unavoidable Minors of Binary 4-Connected Matroids
}

\author{
Susan Jowett
}

\section{VICTORIA UNIVERSITY OF WELLINGTON \\ Te Whare Wānanga o te Ūpoko o te Ika a Māui}

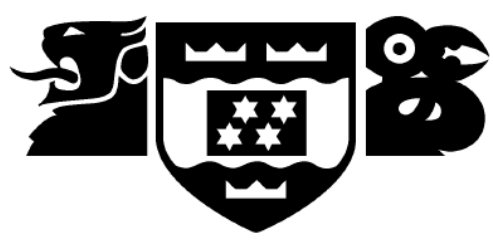

School of Mathematics and Statistics

Te Kura Mātai Tatauranga, Rangahau Pūnaha

\author{
A thesis \\ submitted to the Victoria University of Wellington \\ in fulfilment of the requirements for the degree of \\ Doctor of Philosophy \\ in Mathematics.
}

Victoria University of Wellington 



\begin{abstract}
We show that for every $n \geq 3$ there is some number $m$ such that every 4-connected binary matroid with an $M\left(K_{3, m}\right)$-minor or an $M^{*}\left(K_{3, m}\right)$-minor and no rank- $n$ minor isomorphic to $M^{*}\left(K_{3, n}\right)$ blocked in a path-like way, has a minor isomorphic to one of the following: $M\left(K_{4, n}\right), M^{*}\left(K_{4, n}\right)$, the cycle matroid of an $n$-spoke double wheel, the cycle matroid of a rank- $n$ circular ladder, the cycle matroid of a rank- $n$ Möbius ladder, a matroid obtained by adding an element in the span of the petals of $M\left(K_{3, n}\right)$ but not in the span of any subset of these petals and contracting this element, or a rank- $n$ matroid closely related to the cycle matroid of a double wheel, which we call a non graphic double wheel. We also show that for all $n$ there exists $m$ such that the following holds. If $M$ is a 4-connected binary matroid with a sufficiently large spanning restriction that has a certain structure of order $m$ that generalises a swirl-like flower, then $M$ has one of the following as a minor: a rank- $n$ spike, $M\left(K_{4, n}\right), M^{*}\left(K_{4, n}\right)$, the cycle matroid of an $n$-spoke double wheel, the cycle matroid of a rank- $n$ circular ladder, the cycle matroid of a rank- $n$ Möbius ladder, a matroid obtained by adding an element in the span of the petals of $M\left(K_{3, n}\right)$ but not in the span of any subset of these petals and contracting this element, a rank- $n$ non graphic double wheel, $M^{*}\left(K_{3, n}\right)$ blocked in a path-like way or a highly structured 3-connected matroid of rank $n$ that we call a clam.
\end{abstract}





\section{Acknowledgements}

I would like to thank my wonderfully kind and patient supervisor Geoff for his manipulation without which this thesis would never have been possible. I would also like to thank my amazing pseudo-supervisor Jim for all his help.

Thanks also to my friends, particularly those who made my procrastination so much fun, and my parents and Matt for their never ending love and support. 



\section{Contents}

1 Introduction 1

\begin{tabular}{|lll}
\hline 2 & Background Material & 7
\end{tabular}

$2.1 \quad$ Basic Matroid Theory $\ldots \ldots \ldots$. . . . . . . . . . . 7

2.2 Introduction to Blocking . . . . . . . . . . . . 12

2.3 Introduction to Bridging Sequences $\ldots \ldots \ldots$

2.4 Some Ramsey-Type Results . . . . . . . . . . . . . . . . 15

2.5 A Note on Notation . . . . . . . . . . . . . . . . . . . . 19

3 Flowers and Pseudo-Flowers 21

3.1 Paddles . . . . . . . . . . . . . . . . . . . . . . 24

3.2 Pseudo-flowers . . . . . . . . . . . . . . . . 25

3.3 Swirl-like Pseudo-flowers . . . . . . . . . . . . . . . . . . 28

4 Unavoidable Minors of Binary 3- and 4-connected matroids 41

4.1 Unavoidable Minors of Binary 3-Connected Matroids . . . . . . . 41

4.2 Unavoidable Minors of Binary 4-Connected Matroids . . . . . . . 47

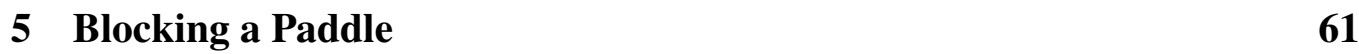

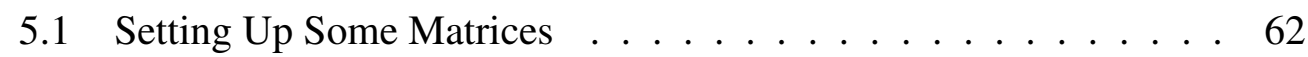

$5.2 \quad$ Blocking $M\left(K_{3, n}^{+}\right) \ldots \ldots \ldots \ldots \ldots \ldots$

5.3 Blocking a Paddle . . . . . . . . . . . . . . . . . 83 
5.4 Proof of Theorem 5.0.1 f . . . . . . . . . . . . . 104

\begin{tabular}{lll}
\hline 6 & Blocking $M^{*}\left(K_{3, n}\right)$ & 107
\end{tabular}

$6.1 \quad$ Crossing Graphs . . . . . . . . . . . . . . . . . . . . . . 109

6.2 Complete Graph . . . . . . . . . . . . . . . . . . . . . 112

6.3 Stars . . . . . . . . . . . . . . . . . . 125

6.4 Paths . . . . . . . . . . . . . . . . . . . . . 139

6.5 Proof of Theorem $6.0 .1 \ldots \ldots$. . . . . . . . . . . . . 139

$\begin{array}{lll}7 & \text { Bridging } M\left(K_{3, n}\right) \text { and } M^{*}\left(K_{3, n}\right) & 141\end{array}$

8 Useful Lemmas For Blocking Swirl-Like Pseudo-Flowers 145

8.1 Crossing Graphs for Swirl-Like Pseudo-Flowers . . . . . . . . . 145

8.2 Structuring the Crossing Elements . . . . . . . . . . . . 152

8.3 Useful Lemmas For Reducing Petals Containing Representatives of Two Blocking Elements . . . . . . . . . . . . . 155

8.4 Useful Lemmas For Reducing Petals Containing Representatives of a Lot of Blocking Elements . . . . . . . . . . . . . 165

\begin{tabular}{|ll|}
\hline 9 & Blocking Swirl-Like Pseudo-Flowers \\
\hline
\end{tabular}

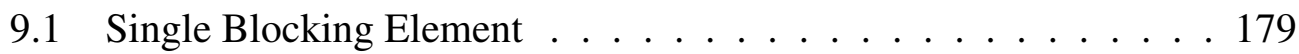

9.2 Stars $\ldots \ldots \ldots \ldots \ldots \ldots \ldots$

9.3 Complete Graphs $\ldots \ldots$. . . . . . . . . . . . . . . . . 196

9.4 Paths . . . . . . . . . . . . . . . . . . . 211

9.5 Proof of Theorem $9.0 .1 \ldots \ldots$. . . . . . . . . . . . 227

10 Summing Up and Future Work 229 


\section{Chapter 1}

\section{Introduction}

In this section we give an overview of the current literature on unavoidable minors and explain they layout of this thesis.

In [7], (1996) Ding, Oporowski, Oxley and Vertigan proved the following theorem.

Theorem 1.0.1. There is a function $f 1.0 .1$ such that the following holds. Suppose that $M$ is a binary 3-connected matroid of rank at least $f_{1.0 .1}(n)$, then $M$ has a minor isomorphic to one of the following:
i) $M\left(K_{3, n}\right)$,
ii) $M^{*}\left(K_{3, n}\right)$,
iii) a rank-n wheel,
iv) a rank-n spike.

We say that these matroids are the unavoidable minors for the class of 3-connected binary matroids.

The notation used for the function in the statement of this theorem is used so that we are able to refer to the function easily later on. A similar notation is used throughout the thesis.

The goal of this thesis is to extend this result to finding the unavoidable minors of binary 4-connected matroids. Unfortunately, due to time constraints, we have not been able to completely resolve this problem but we have made significant 
progress in many of the cases. We want the unavoidable minors of binary 4connected matroids to be close to 4-connected (formally, we want them to be internally 4-connected). In general we are interested in unavoidable minors of matroids with large internally 4-connected sets.

Theorem 1.0.1 is an instance of a series of results that have been obtained for unavoidable minors of graphs and matroids. The simplest example is the following well-known result for graphs (see, for example, [6].

Theorem 1.0.2. For all $n$ there exists an $m$ such that a simple connected graph with at least $m$ edges has either a path of length $n$ or a star on $n$ vertices as a minor.

There is no analogy for matroids, since a star and a path have the same cycle matroid.

The next theorem is also well known and can be extended to matroids.

Theorem 1.0.3. For every $n$ there exists an $m$ such that if $G$ is a loopless 2connected graph on at least $m$ vertices, then $G$ has a cycle with at least $n$ edges or a bond with least $n$ edges.

This result was generalised to matroids by Lovász, Schrijver and Seymour and can be found in [13]. That is, they proved the following.

Theorem 1.0.4. If $M$ is a connected matroid on at least $4^{n}$ elements, then $M$ contains a circuit or a cocircuit with at least $n$ elements.

These results were extended to 3 and 4-connected graphs by Oporowski, Oxley and Thomas [12] (1993); the latter theorem is also proved indirectly by Geelen and Joeris [9] (2008).

Theorem 1.0.5. For every integer $n$ greater than 2 there is an integer $m$ such that every 3-connected graph with more than $m$ vertices contains a minor isomorphic to an $n$-spoke wheel or $K_{3, n}$.

Theorem 1.0.6. For every integer $n$ greater than 2 there is an integer $m$ such that every 4-connected graph with more than $m$ vertices contains a minor isomorphic to $K_{4 . n}$, an n-rung circular ladder, an n-rung Möbius ladder or a double wheel on $n$ vertices. 
In his thesis [16] (2016), Shantanam gave the set of unavoidable minors of large 5-connected graphs, and in 1997 Ding, Oporowski, Oxley, and Vertigan extended Theorem 1.0.1 to non-binary matroids [8]. In general, for a fixed $k$ with $k \geq 2$, we are interested in the question of finding unavoidable minors of large $k$-connected matroids but as connectivity increases the set of unavoidable minors increases and the results increase in difficulty.

A technique for finding unavoidable minors for binary 4-connected matroids is to observe that a 4-connected matroid is also 3-connected and therefore has a minor isomorphic to one of the following:

i) $M\left(K_{3, n}\right)$,

ii) $M^{*}\left(K_{3, n}\right)$,

iii) a rank- $n$ wheel,

iv) a rank- $n$ spike.

Since $M$ is 4-connected, there is a collection of bridging sequences of a 3connected minor of $M$ that gives a 4-connected matroid. Therefore the problem of finding unavoidable minors of binary 4-connected matroids splits into cases, namely, the problem of finding the unavoidable minors when we bridge the 3 separations in $M\left(K_{3, n}\right)$, when we bridge the 3-separations in $M^{*}\left(K_{3, n}\right)$, when we bridge the 3-separations in a rank- $n$ wheel, and when we bridge the 3 -separations in a rank- $n$ spike. Unfortunately due to time constraints we were not able to consider all these problems. We originally believed that had solved the problem of finding the structure when we bridge the 3-separations in $M\left(K_{3, n}\right)$ or in $M^{*}\left(K_{3, n}\right)$ under the assumption that the original matroid did not have a spike minor. However, on closer inspection it turns out that we have instead found the structure when we bridge the 3-separations in $M\left(K_{3, n}\right)$ or in $M^{*}\left(K_{3, n}\right)$ under the assumption that $M^{*}\left(K_{3, n}\right)$ is not blocked in a path-like way. We are close to completing the analysis for bridging a wheel. We have not considered the spike case but do not expect the analysis to be too hard.

The two main results in this thesis are the following theorems. In the statement of the theorems we give matroids that have not yet been defined. The definitions of these matroids are given in Chapter 4. In the second theorem we talk about 
swirl-like pseudo-flowers. These are generalisations of flowers and are studied in Chapter 3.

Theorem. For every $n$, there exists an $m$ such that if $M$ is a 4-connected binary matroid of rank $m$ with an $M\left(K_{3, m}\right)$ or $M^{*}\left(K_{3, m}\right)$ minor, and no minor that isomorphic to $M^{*}\left(K_{3, n}\right)$ blocked in a path-like way, then $M$ must have a minor isomorphic to one of:

i) $N\left(K_{3, n}\right)$,

ii) $M\left(K_{4, n}\right)$,

iii) $\left(N\left(K_{3, n}\right)\right)^{*}$,

iv) $M^{*}\left(K_{4, n}\right)$,

v) the cycle matroid of an n-rung circular ladder,

vi) the cycle matroid of an n-rung Möbius ladder,

vii) the cycle matroid of an n-spoke double wheel,

viii) a rank-n non-graphic double wheel.

The term path-like relates to a crossing graph described in Chapter 6.

Theorem. For every $n$ there is an $m$ such that the following holds. If $M$ is a binary 4-connected matroid with coindependent set $X$ such that $M \backslash X$ has a swirl-like pseudo-flower of order $m$, then $M$ has a minor isomorphic to one of the following:

i) $N\left(K_{3, n}\right)$,

ii) $M\left(K_{4, n}\right)$,

iii) $\left(N\left(K_{3, n}\right)\right)^{*}$,

iv) $M^{*}\left(K_{4, n}\right)$,

v) the cycle matroid of an n-rung circular ladder,

vi) the cycle matroid of an n-rung Möbius ladder,

vii) the cycle matroid of an n-spoke double wheel, 
viii) a rank-n non-graphic double wheel

ix) a rank-n spike.

x) $M^{*}\left(K_{3, n}\right)$ blocked in a path-like way

xi) a rank-n clam.

This theorem is interesting as we believe that when we bridge a wheel we either obtain the cycle matroid of a wheel extended by elements in triangles with the spokes (this is a "clam") or a matroid $M$ with coindependent set $X$ such that $M \backslash X$ has a swirl-like pseudo-flower, $F$. of order $n$ and every 3-separation of $M \backslash X$ displayed by $F$ is blocked by an element of $X$.

The material in this thesis is divided as follows. Chapter 2 gives some basic results on matroids and some Ramsey-type theorems. These results are not new but will be useful in later sections. In Chapter 3 we look at flowers and pseudoflowers. Flowers were first defined by Oxley, Semple and Whittle in [14] (2004) for 3-separations in 3-connected matroids and the results were extended by Aikin and Oxley in [1] (2008) for separations of order $k$ for any $k \geq 2$. In this thesis we use structures called "pseudo-flowers" that are extensions of flowers that allow petals to be both 2-separating and 3-separating. As far as we know these results are new but it is likely that many already exist as folklore. More investigation into pseudo-flowers would have been nice as they are useful and interesting structures. Regrettably we have not had time to do this so the results in this section cover only information required in later sections. In Chapter 4 we give a survey of the matroids that appear as unavoidable minors of binary 3- and 4-connected matroids. We describe and give some natural representations of and state facts about these matroids that will be useful for identifying them later in the thesis. From Chapter 5 onward we finally get into the real content of the thesis and the main results in the remaining chapters are all new. Chapter 5 is dedicated to blocking paddles, Chapter 6 to blocking copaddles and Chapter 7 is a very short chapter that brings the results from Chapters 5 and 6 together to find the unavoidable minors of binary 4-connected matroids with an $M\left(K_{3, n}\right)$ or an $M^{*}\left(K_{3, n}\right)$-minor under the assumption that $M^{*}\left(K_{3, n}\right)$ is not blocked in a path-like way. Chapters 8 and 9 of the thesis relate to blocking swirl-like pseudo-flowers. Chapter 8 sets up tools for blocking swirl-like pseudo-flowers that will be useful in Chapter 9, and Chapter 9 looks at blocking swirl-like pseudo-flowers in detail. The final chapter sums up 
what we have proved in the previous chapters and gives details of future work on this project.

Due to time constraints some of the more obvious proofs in the thesis have been omitted, especially in the later chapters. 


\section{Chapter 2}

\section{Background Material}

In this chapter we give results that will be useful throughout the thesis. The reader is assumed to have a basic knowledge of matroid theory as set forth in [13]. Notation and terminology follow [13].

\subsection{Basic Matroid Theory}

All the results in this section are almost certainly well known and will have proofs in multiple papers.

\subsubsection{Binary Matroids}

A matroid is binary if it is representable over $G F(2)$ The next lemma was proved by Tutte and can be found in [13]

Lemma 2.1.1. A matroid is binary if, and only if, it has no $U_{2,4}$ minor.

It is well known that a simple rank- $n$ binary matroid can be viewed as a restriction of $P G(n-1,2)$. We can at times gain additional information when we consider points of the binary projective space that are not in $M$. For example if $M$ is a matroid with 2-separation $(A, B)$ there is some element of the binary projective space in the span of $A$ and the span of $B$. This element may or may not be in $E(M)$. Matroids allow parallel points and loops but projective spaces do not allow for these. An extended binary projective space of rank $n$ is $P G(n-1,2)$ with as 
many points as needed added in parallel with elements of the projective geometry and loops added as needed. This fits with the fact that binary matroids can be represented by matrices over $G F(2)$, since, in our matrices, we may add repeated columns and zero columns to our heart's desire. If $M$ is a binary matroid with $A \subseteq E(M)$, then $\langle A\rangle$ is the collection of all elements contained in the span of the extended binary projective space of $\operatorname{rank} r(M)$.

\section{Connectivity}

Connectivity plays a huge role in structural matroid theory so what follows is a brief rundown on important facts about connectivity in matroids.

Definition 2.1.2. The connectivity function of a matroid $M$ is a function, $\lambda_{M}$, that maps subsets of $E(M)$ to non-negative integers. We define $\lambda_{M}$ by $\lambda_{M}(X)=$ $r_{M}(X)+r_{M}(E-X)-r(M)$ for any $X \subseteq E(M)$.

Sometimes it is useful to regard $\lambda_{M}$ as being a function on a partition of $E(M)$. We may then refer to $\lambda_{M}(X, Y)$, where $X, Y$ is a partition of $E(M)$, and this is defined by $\lambda_{M}(X, Y)=\lambda_{M}(X)=\lambda_{M}(Y)$. We also abandon the subscript and refer to $\lambda$ instead of $\lambda_{M}$ when the context is clear.

Another useful kind of connectivity function for matroids which will be used later in the thesis is given below.

Definition 2.1.3. Let $M$ be a matroid and $X$ and $Y$ be disjoint subsets of $E(M)$. We define $\kappa_{M}(X, Y)$ by $\kappa_{M}(X, Y)=\min \left\{\lambda_{M}(S): X \subseteq S \subseteq E(M)-Y\right\}$.

Definition 2.1.4. Consider a matroid $M$ and let $X \subseteq E(M)$.

i) We say that $X$ is $k$-separating if $\lambda_{M}(X)<k$.

ii) The partition $(X, E(M)-X)$ is a k-separation if $\lambda(X)<k$ and $|X|, \mid E(M)-$ $X \mid \geq k$.

iii) The partition $(X, E(M)-X)$ is a vertical $k$-separation if $\lambda(X) \leq k$ and $\min \{r(X), r(E(M)-X)\} \geq k$.

iv) The matroid $M$ is $k$-connected if it has no $(k-1)$-separations.

v) The matroid $M$ is vertically $k$-connected if it has no vertical $(k-1)$ separations. 
vi) A $k$-separation $(X, Y)$ is minimal if $\min \{|X|,|Y|\}=k$.

vii) A matroid is internally $(k+1)$-connected if it has no non-minimal $k$ separations.

viii) A $k$-separation $(X, E(M)-X)$ is exact if $\lambda(X)=k-1$.

ix) A matroid is connected if it is 2-connected.

The following lemma gives two well-known facts about connectivity functions that will be used freely throughout this thesis. We say that a set function $f$ is normalised if $f(\emptyset)=0$.

Lemma 2.1.5. Let $M$ be a matroid with connectivity function $\lambda$.

i) The connectivity function of $M$ is normalised, symmetric and submodular.

ii) $\lambda_{M}=\lambda_{M^{*}}$.

iii) If $N$ is a minor of $M$, then $\lambda_{M}(X) \geq \lambda_{N}(X)$ for any $X \subseteq E(N)$.

The next result is a trivial corollary of the submodularity of the connectivity functions.

Lemma 2.1.6. Let $M$ be a matroid and let $X, Y \subseteq E(M)$ such that $\lambda(X), \lambda(Y) \leq$ 2. If $\lambda(X \cup Y) \geq 2$, then $\lambda(X \cap Y) \leq 2$. In particular, if $M$ is 3-connected and $\lambda(X)=\lambda(Y)=2$ then

i) if $|X \cap Y| \geq 2$ then $X \cup Y$ is 3-separating, and

ii) if $|E(M)-(X \cup Y)| \geq 2$, then $X \cap Y$ is 3-separating.

An application of Lemma 2.1.6 will be referred to as an application of uncrossing.

We often want to be able to keep connectivity in a minor of a matroid. This makes the following lemma of Tutte [17] very useful.

Lemma 2.1.7. Let $M$ be a connected matroid and $e \in E(M)$. Then either $M \backslash e$ or $M / e$ is connected. 
Recall that a parallel pair is a 2-element circuit and a series pair is a 2-element cocircuit. We say that a matroid $M$ is 3-connected up to parallel classes if the simplification of $M$ is 3-connected and we say that $M$ is 3-connected up to series classes if the co-simplification of $M$ is 3-connected. The following lemma can be found in [2].

Lemma 2.1.8 (Bixby's Lemma). Let e be an element of a 3-connected matroid M. Either $M \backslash e$ or $M /$ e has no non-trivial 2-separation. Moreover, in the first case the cosimplification of $M \backslash e$ is 3-connected, while in the second case the simplification of $M / e$ is 3-connected.

\section{Tutte's Linking Theorem}

Let $M$ be a matroid and $X$ and $Y$ disjoint subsets of $E(M)$. Recall $\kappa_{M}(X, Y)=$ $\min \{\lambda(A): X \subseteq A \subseteq E(M)-Y\}$. The following theorem is a generalisation of Menger's Theorem for matroids.

Theorem 2.1.9 (Tutte's Linking Theorem). Let $M$ be a matroid and let $X$ and $Y$ be disjoint subsets of $E(M)$ and suppose $\lambda_{M}(X)=\lambda_{M}(Y)=\kappa_{M}(X, Y)$. There exists a minor $N$ on $X \cup Y$ such that $\lambda_{N}(X)=\kappa_{M}(X, Y)$.

Now consider Tutte's Linking Theorem together with the lemma below.

Lemma 2.1.10. Let $N$ be a minor of a matroid $M$ and let $X \subseteq E(N)$. If $\lambda_{M}(X)=$ $\lambda_{N}(X)$, then $M|X=N| X$.

This gives the following result, which we use frequently throughout this section. For ease of reference and since this follows almost immediately from Tutte's Linking Theorem we shall often say "by Tutte's Linking Theorem" as opposed by "by Lemma 2.1.11,.

Theorem 2.1.11. Let $M$ be a matroid and let $X$ and $Y$ be disjoint subsets of $E(M)$ and suppose $\lambda_{M}(X)=\lambda_{M}(Y)=\kappa_{M}(X, Y)$. Then there exists a minor $N$ on $X \cup Y$ such that $\lambda_{N}(X)=\kappa_{M}(X, Y)$. Moreover, $N|X=M| X$ and $N|Y=M| Y$. 


\section{Local Connectivity}

Definition 2.1.12. Let $M$ be a matroid with ground set $E$. The local connectivity between two disjoint sets $X, Y \subseteq E$, denoted $\sqcap_{M}(X, Y)$, is defined by $\sqcap_{M}(X, Y)=r(X)+r(Y)-r(X \cup Y)$.

As usual we abandon the subscript where context allows. It is a trivial observation that when $X$ and $Y$ are disjoint $\sqcap_{M}(X, Y)=\lambda_{M \mid(X \cup Y)}(X)$.

Lemma 2.1.13. Let $M$ be a matroid and $X_{1}, X_{2}, Y_{1}, Y_{2} \subseteq E(M)$. If $X_{1} \subseteq X_{2}$ and $Y_{1} \subseteq Y_{2}$ then $\sqcap\left(X_{1}, Y_{1}\right) \leq \sqcap\left(X_{2}, Y_{2}\right)$.

When $M$ is represented over a field, $\sqcap(X, Y)$ is the rank of the intersection of the span of $X$ and the span of $Y$ in the underlying projective space over that field.

We use $\sqcap_{M}^{*}(X, Y)$ to denote $\sqcap_{M^{*}}(X, Y)$.

\section{Guts and Coguts Elements}

Let $M$ be a matroid on groundset $E$. Let $X \subseteq E$ and $e \notin E$. If $e \notin X$, it is easy to see that $e \in \operatorname{cl}_{M}(X)$ if and only if $e \notin \operatorname{cl}_{M^{*}}(E-(X \cup e))$.

Lemma 2.1.14. Let $M$ be a matroid, let $X \subseteq E(M)$, and let $e \in E(M)-X$. Then $\lambda_{M / e}(X)<\lambda_{M}(X)$ if, and only if, $e \in c_{M}(X)$ and e is not a loop.

When it is clear from the context we abbreviate $\operatorname{cl}_{M^{*}}(X)$ to $\mathrm{cl}^{*}(X)$.

Definition 2.1.15. Let $(X, Y)$ be an exact $k$-separation of a matroid $M$. An element $e$ is in the guts of $(X, Y)$ if $e \in \operatorname{cl}_{M}(X-e)$ and $e \in \operatorname{cl}_{M}(Y-e)$. Dually, $e$ is in the coguts of $(X, Y)$ if $e \in \mathrm{cl}_{M}^{*}(X-e)$ and $e \in \mathrm{cl}_{M}^{*}(Y-e)$.

\section{$M\left(K_{4}\right)$ Minors}

The following lemma from [15] is exceedingly useful and will be used frequently throughout the thesis.

Lemma 2.1.16. Let $\{a, b, c\}$ be elements of a 3-connected binary matroid $M$ with $r(M) \geq 3$. Then $M$ has an $M\left(K_{4}\right)$-minor using $\{a, b, c\}$. 


\subsection{Introduction to Blocking}

Definition 2.2.1. Let $M$ be a matroid and $x \in E(M)$. Let $(A, B)$ be a $k$-separation of $M \backslash x$. We say that $(A, B)$ is blocked by $x$ in $M$, or $x$ blocks $(A, B)$ in $M$, if $\left.\lambda_{M}(A \cup\{x\}), B\right)=\lambda_{M}(A, B \cup\{x\}) \neq \lambda_{M \backslash x}(A, B)$.

Let $M$ be a matroid, $X \subseteq E(M)$ and $N=M \backslash X$. For $X^{\prime} \subset X$ we may denote the matroid $M \backslash\left(X-X^{\prime}\right)$ by $N+X^{\prime}$. We say $x$ blocks the separation $(A, B)$ of $N$ if $(A, B)$ is blocked by $x$ in $N+x$. If $(A, B)$ is not a $k$-separation in $M$ but is in $M \backslash x$ then we say that deleting $x$ unblocks the $k$-separation $(A, B)$.

Lemma 2.2.2. Let $M$ be a matroid and $N$ a minor of $M$. Let $(A, B)$ be a $k$ separation in $N$. An element $x \in(E(M)-E(N))$ blocks $(A, B)$ if, and only if, $x$ is not a coloop in $N+x$ and $x \notin \mathrm{cl}_{N+x}(A)$ and $x \notin \mathrm{cl}_{N+x}(B)$.

Proof. Say $x$ blocks $(A, B)$. Then

$$
\lambda_{N+x}(A \cup x, B)=\lambda_{N+x}(A, B \cup x)=\lambda_{N}(A, B)+1 .
$$

Therefore

$$
r_{N+x}(A \cup x)+r_{N+x}(B)-r(N+x)=r_{N}(A)+r_{N}(B)-r(N)+1 .
$$

As $x$ is a blocking element $r_{N+x}(B)=r_{N}(B)$ and so, as the rank function of a matroid is integral, either $r(N+x)<r(N)$, a contradiction, or

$$
r_{N+x}(A \cup x)=r_{N}(A)+1=r_{N+x}(A)+1 .
$$

Similarly

$$
r_{N+x}(B \cup x)=r_{N}(B)+1=r_{N+x}(B)+1 .
$$

This means that $x \notin \mathrm{cl}_{N+x}(A)$ and $x \notin \mathrm{cl}_{N+x}(B)$.

Finally a simple rank argument shows that if $x$ were a coloop in $N+x$ we would have $\lambda_{N+x}(A \cup x)=\lambda_{N}(A)$. Therefore $x$ is not a coloop in $N+x$.

The other direction is relatively similar and is left to the reader. 


\subsection{Introduction to Bridging Sequences}

Some background on bridging sequences can be found in [10] but all necessary definitions and results can also be found below.

Definition 2.3.1. Let $M$ be a matroid. Consider an exact $k$ separation $(X, Y)$ in the matroid $N=M \backslash D / C$. We say that $(X, Y)$ is bridged in $M$ if $\kappa_{M}(X, Y) \geq k$.

Let $N=M \backslash D / C$ and suppose $X \subseteq C \cup D$. We shall use $N[X]$ to denote the matroid $M \backslash(D-X) /(C-X)$.

Definition 2.3.2. Let $V=v_{1}, \ldots, v_{n}$ be an ordered collection of elements of $E(M)-E(N)$ and let $(X, Y)$ be a $k$-separation of $N$ that is bridged in $M$. Let $S=\left\{v_{i}: i\right.$ odd and $\left.i \in\{1, \ldots, n\}\right\}$ and $T=\left\{v_{i}: i\right.$ even and $\left.i \in\{1, \ldots, n\}\right\}$. Then $V$ is a bridging sequence for the $k$-separation $(X, Y)$ if the following hold:

i) There are sets $C, D$ such that $\{C, D\}=\{S, T\}$ such that $D$ is an independent set and $C$ is a coindependent set and $N=M \backslash D / C$,

ii) if $i \in\{1, . ., n\}$, then $\lambda_{M}\left(X \cup\left\{v_{1}, \ldots, v_{i}\right\}, Y \cup\left\{v_{i+1} \ldots, v_{n}\right\}\right)=k$,

iii) if $v_{i} \in D$, then $\lambda_{M \backslash v_{i}}\left(X \cup\left\{v_{1}, \ldots, v_{i-1}\right\}, Y \cup\left\{v_{i+1} \ldots, v_{n}\right\}\right)=k-1$, and

iv) if $v_{i} \in C$, then $\lambda_{M / v_{i}}\left(X \cup\left\{v_{1}, \ldots, v_{i-1}\right\}, Y \cup\left\{v_{i+1} \ldots, v_{n}\right\}\right)=k-1$.

We call $D$ the delete set for $V$ and $C$ the contract set for $V$.

Definition 2.3.3. If a $k$-separation $(X, Y)$ of $N$ is bridged in a matroid $M$, then we say that $M$ is a bridging matroid for $(X, Y)$. If no proper minor of $M$ exists in which $(X, Y)$ is bridged, then $M$ is a minimal bridging matroid for $(X, Y)$. If $V$ is a bridging sequence for $(X, Y)$ that is contained in a minimal bridging matroid then we call $V$ a minimal bridging sequence.

The next few lemmas can be found in [10].

Lemma 2.3.4. Let $M$ be a $k$-connected matroid that contains a $k$-separation $(A, B)$ and let $N$ be a minor of $M$ containing a $k$-separation. Then there is a minor of $M$ that contains a minimal bridging sequence for $(A, B)$ in $N$. 
Lemma 2.3.5. Let $V$ be a minimal bridging sequence with delete set $D$ and contract set $C$ for the $k$-separation $(X, Y)$ in $N$. Let $M$ be a minimal bridging matroid for $N$. If $x \in D$, then $M / x$ is not $k$-connected and if $x \in C$ then $M \backslash x$ is not $k$ connected.

Lemma 2.3.6. Let $V=\left(v_{0}, \ldots, v_{n}\right)$ be a bridging sequence for the $k$-separation $(X, Y)$ in $N$.

i) If $v_{i}$ is a delete element of $V$, then $v_{i} \notin \mathrm{cl}_{N\left[v_{0}, \ldots, v_{i}\right]}(Y)$.

ii) If $v_{i}$ is a contract element of $V$, then $v_{i} \notin \mathrm{cl}_{N\left[v_{0}, \ldots, v_{i}\right]}^{*}(Y)$.

Lemma 2.3.7. Let $V=\left\{v_{0}, \ldots, v_{n}\right\}$ be a bridging sequence for the $k$-separation $(X, Y)$ in $N$. Let $i<n$. Then, in $N\left[v_{0}, \ldots, v_{i}\right]$, we have $v_{i} \in \operatorname{cl}\left(X \cup\left\{v_{0}, \ldots, v_{i-1}\right\}\right)$ and $v_{i} \in \mathrm{cl}^{*}\left(X \cup\left\{v_{0}, \ldots, v_{i-1}\right\}\right)$.

Lemma 2.3.8. Let $(A, B)$ be a k-separation in matroid $N$ that is bridged by a bridging sequence $\left\{v_{0}, \ldots, v_{n}\right\}$ that starts and finishes with a delete element. Then $\left(A \cup\left\{v_{0}, \ldots v_{n-1}\right\}, B\right)$ is a k-separation in $N\left[v_{0}, \ldots, v_{n-1}\right]$ that is blocked by $v_{n}$. Moreover, $v_{n-1}$ is in the coguts of $(A, B)$ in $N\left[v_{0}, \ldots, v_{n-1}\right]$.

Proof. The first part of the lemma is obvious. To show $v_{n-1}$ is in the coguts of $(A, B)$ in $N\left[v_{0}, \ldots, v_{n-1}\right]$ observe that, since $\lambda_{N}(A)=r_{N}(A)+r_{N}^{*}(A)-|A|=$ $\lambda_{N}(B)=\lambda_{N}\left[v_{n-1}\right]\left(A \cup v_{n-1}\right)=r\left(A \cup\left\{v_{n-1}\right\}\right)+r^{*}\left(A \cup v_{n-1}\right)-|A|-1$, the element $v_{n-1}$ is either in the closure or the coclosure of $A$. Similarly, $v_{n-1}$ is either in the closure or the coclosure of $B$. Suppose that $v_{n-1} \in \operatorname{cl}(B)$. We know that $v_{n} \in \operatorname{cl}(B \cup$ $\left.\left\{v_{n-1}\right\}\right)$ so this means $v_{n} \in \operatorname{cl}(B)$, a contradiction in $N\left[v_{n-1}, v_{n}\right]$. Suppose that $v_{n-1} \in \operatorname{cl}(A)$. If this happens then $\lambda_{N\left[v_{n-1}\right]}(A) \neq \lambda_{N\left[v_{n-1}\right]}(B)$, a contradiction.

Note that if an element $x$ is in the coguts of a $k$-separation $(A, B)$, then $(A, B)$ is a $k-1$-separation in $M \backslash x$.

Lemma 2.3.9. Let $(A \cup\{x\}, B)$ be a 3-separation of matroid $N$ that is blocked by a single extension element $b$. Suppose that $x$ is in the coguts of $(A, B)$ and suppose that $A$ is a 3-separating triad, $\left\{t_{1}, t_{2}, t_{3}\right\}$. There is a minor $N^{\prime}$ of $N$ with groundset $B \cup\left\{t_{i}, t_{j}, x\right\}$ so that $N^{\prime}|B=N| B$, and $\left\{t_{i}, t_{j}, x\right\}$ is a 3-separating triad that is blocked by $b$ for some $i, j \in\{1,2,3\}$. 
Proof. Suppose that when we contract $t_{1}$ the element $t_{2}$ is in the closure of $B$, then when we contract $t_{2}$ we have $t_{1} \in \operatorname{clN} / t_{2}(B)$. Now consider contracting $t_{3}$. Say $t_{i} \in \mathrm{cl}_{N / t_{3}}(B)$, for $i \in\{1,2\}$. Then $r_{N}\left(B \cup\left\{t_{1}, t_{2}, t_{3}\right\}\right)=r_{N}\left(B \cup\left\{t_{3}\right\}\right)$ so $\lambda_{N \backslash x}\left(\left\{t_{1}, t_{2}, t_{3}\right\} \cup B\right)=2$, a contradiction.

We now show that if, when we contract $t_{3}$, neither of $t_{1}$ or $t_{2}$ is in the closure of $B$, then $\left\{t_{1}, t_{2}, x\right\}$ is a 3 -separating triad in $N / t_{3}$ that is blocked by $b$. First $r_{N / t_{3}}(B)=r_{N}\left(B \cup t_{3}\right)-1$ and $r\left(N / t_{3}\right)=r\left(B \cup\left\{t_{1}, t_{2}, t_{3}, x\right\}\right)-1=r\left(B \cup t_{3}\right)-1$ (since $x$ is not a coloop). Therefore, $B$ is a hyperplane in $N / t_{3}$ and so $\left\{t_{1}, t_{2}, x\right\}$ is a triad. It is clear that $\left\{t_{1}, t_{2}, x\right\}$ is 3 -separating and $\left(B,\left\{t_{1}, t_{2}, x\right\}\right)$ is a 3 -separation in $N^{\prime}$ blocked by $b$.

\subsection{Some Ramsey-Type Results}

Ramsey's Theorem tells us that any sufficiently large graph either has a clique or an independent set of size $n$ as a minor. In general, Ramsey-theoretic results are of the following form: Let $S$ be a substructure of interesting form $A$, then any sufficiently large structure has a substructure of $|S|$ with form $A$. In this section we give some Ramsey theoretic results that will be useful throughout the thesis.

The following lemma can be found in [13].

Lemma 2.4.1. There is a function $\int 2.4 .1$ such that a connected matroid $M$ with rank at least $f_{2.4 .1}(n)$ contains a circuit on n elements or cocircuit on n elements.

The next lemma can be found in Chapter 9 of [6].

Lemma 2.4.2. There is a function $\int_{[2.4 .2}$ such that if $G$ is a simple connected graph with at least $\int 2.4 .2(n)$ vertices, then $G$ contains, as an induced subgraph, a graph isomorphic to $K_{n}, K_{1, n}$, or a path of length $n$.

We assume the reader has a basic knowledge of hypergraphs.

Definition 2.4.3. A hypergraph $H$ is connected if there is a walk between every pair of distinct vertices in $H$. A matching in a hypergraph is a set of pairwise disjoint nonempty hyperedges.

The proof of the following well-known theorem is routine and is omitted. 
Lemma 2.4.4. There is a function $\sqrt{2.4 .4}$ such that the following holds. Let $H$ be a connected hypergraph with at least $\int 2.4 .4(n, m, k)$ elements. If every edge of $H$ has size at most $k$, then either $H$ has a vertex of degree greater than $m$ or $H$ has a matching using $n$ edges.

We now take a brief detour to define "block decompositions" of matrices. These will also be used in later chapters of the thesis.

Definition 2.4.5. Let $A$ be a matrix with a set $R=\left\{r_{1}, \ldots, r_{m}\right\}$ of rows and $C=$ $\left\{c_{1}, \ldots, c_{n}\right\}$ of columns. A block decomposition of $A$ is a partition, $\widetilde{A}$, of $A$ into submatrices such that the following hold.

1. If $B$ is a submatrix of $A$ in $\widetilde{A}$ then all rows of $B$ are consecutive in $A$ and all columns of $B$ are consecutive in $A$.

2. If $B$ is in $\widetilde{A}$ and the rows of $B$ are labelled by $r_{i}, \ldots, r_{k}$ then for any $C \in \widetilde{A}$ with a row labelled by an element of $\left\{r_{i}, \ldots, r_{k}\right\}, C$ contains exactly rows labelled by $r_{i}, \ldots, r_{k}$.

3. If $B$ is in $\widetilde{A}$ and the columns of $B$ are labelled by $c_{i}, \ldots, c_{k}$ then for any $C \in \widetilde{A}$ with column labelled by an element of $\left\{c_{i}, \ldots, c_{k}\right\}, C$ contains exactly rows labelled by $r_{i}, \ldots, r_{k}$.

Definition 2.4.6. We say that a matrix $A$ is almost diagonal if $A$ has a block decomposition so that the only non-zero blocks are the diagonal blocks and the diagonal blocks are of the form $(1,1, \ldots, 1)^{T}$. We say that $A$ is $n$-block almost diagonal if $A$ is almost diagonal and the block decomposition has $n$ diagonal blocks.

We assume the following theorem is well known. Regardless the proof is straightforward and is omitted

Lemma 2.4.7. There is a function $\sqrt{2.4 .7}$ such that the following holds. Let $M$ be a binary matrix containing at most $k$ ones in a column and exactly one 1 in a row. Suppose $M$ has at least $\int[2.4 .7(n)$ columns. Then there is a submatrix of $M$ obtained by deleting columns and resulting zero rows that has $m$ consecutive rows that form a column-permuted n-block almost diagonal matrix.

The next lemma follows easily. 
Lemma 2.4.8. There is a function $\sqrt{2.4 .8}$ such that if $M$ is a matrix with at least $\int 2.4 .8(n, k)$ columns and exactly one 1 in each column, then there is either a submatrix of $M$ obtained by deleting columns and zero rows and permuting columns that is an almost-diagonal matrix with at least $n$ elements, or there is a row containing at least $k$ elements.

Proof. This follows by letting $\int \frac{12.4 .8}{(n, k)}=\int_{2.4 .4}\left(\int_{22.4 .7}(n), k\right)$.

Lemma 2.4.9. There is a function $\sqrt{2.4 .9}$ with the following property. Suppose $n$ is an integer greater than 2 and $M$ is a matrix over $G F(2)$ with at least $\sqrt{2.4 .9}(n)$ columns. Suppose that every column of $M$ contains at least two ones and no two columns are identical. By permuting columns, deleting rows and deleting columns we can find a submatrix with at least $n$ rows of one of the following forms:

$$
\left(\begin{array}{l}
1 \\
1 \\
1 \\
1 \\
1 \\
1 \\
\vdots \\
1
\end{array}\right),\left(\begin{array}{ccccccc}
1 & 1 & 1 & 1 & 1 & \ldots & 1 \\
1 & 0 & 0 & 0 & 0 & \ldots & 0 \\
0 & 1 & 0 & 0 & 0 & \ldots & 0 \\
0 & 0 & 1 & 0 & 0 & \ldots & 0 \\
0 & 0 & 0 & 1 & 0 & \ldots & 0 \\
0 & 0 & 0 & 0 & 1 & \ldots & 0 \\
\vdots & \vdots & \vdots & \vdots & \vdots & \ldots & \vdots \\
0 & 0 & 0 & 0 & 0 & \ldots & 1
\end{array}\right),\left(\begin{array}{cccccc}
1 & 0 & 0 & 0 & \ldots & 0 \\
1 & 1 & 0 & 0 & \ldots & 0 \\
0 & 1 & 1 & 0 & \ldots & 0 \\
0 & 0 & 1 & 1 & \ldots & 0 \\
0 & 0 & 0 & 1 & \ldots & 0 \\
\vdots & \vdots & \vdots & \vdots & \ldots & \vdots \\
0 & 0 & 0 & 0 & \ldots & 1 \\
0 & 0 & 0 & 0 & \ldots & 1
\end{array}\right)
$$

where $A_{i}$ denotes an $a_{i} \times b_{i}$ matrix with a 1 in every row and every column.

Proof. First we obtain a hypergraph $H$ from any matrix $M$. To do this let the rows be the vertices of the graph and, if column $c_{i}$ had a 1 in rows $r_{j_{1}}, \ldots, r_{j_{k}}$ then there is an edge in $H$ incident with $r_{j_{1}}, \ldots, r_{j_{k}}$. We may assume that the hypergraph has 
at most $n$ connected components otherwise we are in the case where the matrix is of the following form.

$$
\left(\begin{array}{ccccc}
A_{1} & 0 & 0 & \ldots & 0 \\
0 & A_{2} & 0 & \ldots & 0 \\
0 & 0 & A_{3} & \ldots & 0 \\
\vdots & \vdots & \vdots & \ddots & \vdots \\
0 & 0 & 0 & \ldots & A_{n}
\end{array}\right)
$$

This means that at least one component, $H_{1}$ has at least $\frac{|V(H)|}{n}$ vertices.

If there is an edge incident with at least $n$ vertices then there is a submatrix of $M$ that is a column of $1^{\prime} s$, and if there is a vertex that meets at least $k \geq \sqrt{2.4 .8}(n, n)$ edges then, by Lemma 2.4.8, we get a submatrix of $M$ of the following form.

$$
\left(\begin{array}{ccccccc}
1 & 1 & 1 & 1 & 1 & \ldots & 1 \\
1 & 0 & 0 & 0 & 0 & \ldots & 0 \\
0 & 1 & 0 & 0 & 0 & \ldots & 0 \\
0 & 0 & 1 & 0 & 0 & \ldots & 0 \\
0 & 0 & 0 & 1 & 0 & \ldots & 0 \\
0 & 0 & 0 & 0 & 1 & \ldots & 0 \\
\vdots & \vdots & \vdots & \vdots & \vdots & \ldots & \vdots \\
0 & 0 & 0 & 0 & 0 & \ldots & 1
\end{array}\right)
$$

Assume that every vertex of $H_{1}$ has degree less than $k$, and let $v$ be a vertex of $H_{1}$. We call this vertex layer 0 . Let layer 1 be the set of all edges incident with $v$ that meet $v$ and all vertices incident with these edges that are not in layer 0 . Note that layer 1 has size at most $k n$. Let layer $i$ be the set of all edges incident with a vertex in layer $i-1$ that are not in layer $i-1$ and all vertices that meet an edge of layer $i$ that are not in layer $i-1$. This has at most $\left|L_{i-1}\right| k n$ vertices. If $f_{? ?}(n) \geq(k n)^{n}$ then there are at least $n$ layers in $H$. Take a vertex $v_{i}$ from layer $L_{i}$ that is incident with an edge $e_{i+1}$. Consider an edge $e_{i} \neq e_{i+1}$ that is incident with $v$ and consider some element of $L_{i-1}$ that meets $e_{i}$. The collection of vertices and edges obtained in this way forms a path.

Lemma 2.4.10. Suppose $A$ is a $\sqrt{2.4 .10}(n) \times \sqrt{2.4 .10}(m)$ matrix with at least one 1 in every row and every column, and no column containing more than $k$ ones. Then there is a large submatrix of A that is either a column of $k$ ones or a permutation 
of $I_{l}$ where $l=\min \{m, n\}$.

Proof. This follows immediately from Lemma 2.3 of [7].

The next result is trivial.

Lemma 2.4.11. There is some function $\int[2.4 .11(n, k)$ such that if $S$ is a sequence of length $f_{[2.4 .11}(n, k)$ in which every entry is taken from the set $\{1, \ldots, k\}$ then there is a subsequence of $S$ of size at least $n$ in which all elements take the same value.

\subsection{A Note on Notation}

For a set $A$, we use $\mathscr{P}(A)$ to denote the powerset of $A$. If $A$ is a set of sets, $S_{1}, \ldots, S_{n}$ then $\cup A$ denotes $S_{1} \cup \ldots \cup S_{n}$ (see, for example [11][pg 12], and $A-S-i$ denotes $\left\{S_{1}, \ldots, S_{i-1}, S_{i+1}, \ldots, S_{n}\right\}$. If $A$ is an ordered set such that $A=\left(S_{1}, \ldots, S_{n}\right)$ then $A-S_{i}$ denotes $\left(S_{1}, \ldots, S_{i-1}, S_{i+1}, \ldots, S_{n}\right)$.

If $F$ is a set, then an $F$-matrix is a matrix that takes its entries from $F$. If $A$ and $B$ are two matrices with the same number of rows, then we use $A \frown B$ to denote the matrix obtained by augmenting the matrix $A$ by the matrix $B$. When we represent matroids we will frequently use a reduced standard representation (see [13] (pg 78)).

When we are considering a matroid $M$ with a fixed basis $B$ we use $F_{B}(x)$ to denote the fundamental circuit of $x$ with respect to $B$. When the basis in question is clear we may abbreviate this to $F(x)$. We refer to the elements of $F(x)$ as the representatives of the element $x$.

A lot of this thesis relies on pivoting on matrices. There will often be a sequence of many pivots where at each stage the matrix we pivot on changes due to previous pivots. It becomes extremely annoying to have to name each matrix in the sequence. Therefore, when the matrix we are discussing is clear, we use $M_{i, j}$ to denote the $(i, j)^{t h}$ entry of of the matrix in question. This notation is subideal but seems better than having to name hundreds of individual matrices.

Let $S=a_{1}, a_{2}, \ldots, a_{n}, a_{1}$ be a cyclic order in which one starts from $a_{1}$ and, moving clockwise, next comes to $a_{2}$ then $a_{3}$ and so on. We use $\left[x_{1}, x_{2}, \ldots, x_{i}\right]_{a_{j}}$ to denote the fact that, if we start from $a_{j}$ and move around $S$ in a clockwise direction, we first see $x_{1}$ then $x_{2}$ and so on. Note that $x_{i}$ and $x_{i+1}$ need not be consecutive in 
the cyclic order. Again this is not notation that I am particularly happy with, but cyclic orders seem particularly nasty to talk about!

The matroids in this thesis are generally binary. To reduce the number of lines we need and make our matroid drawings looks a little less daunting, when we give a drawing of a matroid we may sometimes omit dependencies forced by the fact the matroid is binary if we have enough information from the rest of the picture to fully determine the matroid.

We make the global assumption here that, unless otherwise stated, $t \in \mathbb{Z}_{\geq 4}$. 


\section{Chapter 3}

\section{Flowers and Pseudo-Flowers}

Definition 3.0.1. Let $M$ be a matroid and $F$ be a partition of $E(M)$ into $\left(P_{1}, \ldots, P_{n}\right)$. Then, for $k \in \mathbb{Z}_{\geq 2} F$ is a $(k+1)$-flower of $M$ if the following hold.

1. If $n>1$ then $\lambda\left(P_{i}\right)=k$ for all $i \in\{1, \ldots, n\}$,

2. If $n>2$, then $\lambda\left(P_{i} \cup P_{i+1}\right)=k$ for $i \in\{1, \ldots, n\}$ and addition of subscripts is modulo $n$,

3. if $(X, Y)$ is a 2-separation of $M$, then for some petal $P_{i}$, either $X$ or $Y$ is a subset of $P_{i}$ A flower is a $k$-flower for some $k \geq 2$

We call the elements of $F$ the petals of $F$. We say that a petal $P_{i}$ of $F$ is proper if $P_{i} \subseteq \operatorname{cl}\left(E(M)-P_{i}\right)$. A set $S$ of petals of $F$ is consecutive if, for petals $P_{i}, P_{j} \in S$ either $P_{k} \in S$ for all $k$ such that $[i, k, j]_{i}$, or $P_{k} \in S$ for all $k$ such that $[j, k, i]_{j}$.

Since $F$ is an ordered set we can talk about subsets of $F$. We use $F^{\prime} \subseteq F$ to denote a flower $F^{\prime}$ where the petals of $F^{\prime}$ are a subset of $F$ and the order in which the petals occur in $F$ is preserved in $F^{\prime}$.

The class of $(k+1)$-flowers splits into two subclasses: anemones, and daisies.

Definition 3.0.2. Let $F$ be a $(k+1)$-flower. We call $F$ an anemone if $\cup_{i \in I} P_{i}$ is exactly $k$-separating for any $I \subsetneq\{1, \ldots, n\}$. We call $F$ a daisy if $\cup_{i \in I} P_{i}$ is exactly $k$-separating if, and only if, non-empty $I \subsetneq\{1, \ldots, n\}$, and the members of $I$ form a consecutive set modulo $n$. 
Theorem 1.1 of [1] proves that all flowers are either anemones or daisies. An alternative way of viewing these classes is in terms of the local connectivity between petals. Recall that the local connectivity of two disjoint sets, $X$ and $Y$ is defined by $\sqcap(X, Y)=r(X)+r(Y)-r(X \cup Y)$. If we want a definition of an anemone or daisy in terms of local connectivity to make sense we need the following lemma which can be found in [1].

Lemma 3.0.3. Let $\left(P_{1}, \ldots, P_{n}\right)$ be a flower in a matroid $M$ with at least five petals. If $\sqcap\left(P_{1}, P_{2}\right)=k$ then $\sqcap\left(P_{i}, P_{i+1}\right)=k$ for any $i \in\{1, \ldots, n\}$. Moreover, if $\sqcap\left(P_{1}, P_{3}\right)=$ $k^{\prime}$ then $\sqcap\left(P_{i}, P_{j}\right)=k^{\prime}$ for all $i, j \in\{1, \ldots, n\}$ where $i \neq j+1$ and $j \neq i+1$.

In Lemma ?? we introduced the condition that $M$ has at least five petals. This is because we want to remove the possibility of Vámos-like flowers. These have four petals and, since they are non-binary, are of no interest to us. Recall that a connectivity function is self-dual. It is then easy to see that if $F$ is a flower in $M$, then $F$ is also a flower in $M^{*}$. Therefore Lemma ?? holds when we replace $\sqcap$ by $\Pi^{*}$.

We will describe a flower in terms of three parameters.

Definition 3.0.4. Let $M$ be a matroid. A $(k+1)$-flower, $\left(P_{1}, \ldots, P_{n}\right)$, of $M$ with at least 5 petals is a $(\mu, v, \xi)$-flower in $M$ if $\sqcap\left(P_{1}, P_{3}\right)=\mu$, if $\sqcap^{*}\left(P_{1}, P_{3}\right)=v$ and if $\sqcap\left(P_{1}, P_{2}\right)-\sqcap\left(P_{1}, P_{3}\right)=\xi$.

We will shortly prove that once we know any three of $k, \mu, v, \xi$, the fourth is fixed.

Lemma 3.0.5. Let $F$ be a $(k+1)$-flower that is a $(\mu, v, \xi)$-flower of a matroid $M$ and suppose $F$ has at least 5 petals. Then $F$ is a $(v, \mu, \xi)$-flower of $M^{*}$.

Proof. It is trivial to see that $F$ is a flower in $M^{*}$ and that $F^{*}$ is a $(v, \mu, \sigma)$ flower for some $\sigma$. What remains to prove is that $\sigma=\xi$, that is that $\sqcap\left(P_{1}, P_{2}\right)-\sqcap\left(P_{1}, P_{3}\right)=\Pi^{*}\left(P_{1}, P_{2}\right)-\Pi^{*}\left(P_{1}, P_{3}\right)$. To do this notice that $\sqcap(A, B)=$ $\lambda_{M \backslash E-\{A \cup B\}}(A, B)$ so $\Pi^{*}(A, B)=\lambda_{M / E-\{A \cup B\}}(A, B)$.

Claim 3.0.6. Let $F=\left(P_{1}, P_{2}, P_{3}, P_{4}, P_{5}\right)$ be a $(\mathrm{k}+1)$-flower with five petals such that $\sqcap\left(P_{1}, P_{3}\right)=\sqcap\left(P_{2}, P_{4}\right)$. Then $\sqcap\left(P_{1}, P_{2}\right)-\sqcap\left(P_{1}, P_{3}\right)=\Pi^{*}\left(P_{1}, P_{2}\right)-$ $\sqcap^{*}\left(P_{1}, P_{3}\right)$. 
Proof. Consider $\Pi^{*}\left(P_{1}, P_{3}\right)=\lambda_{M / P_{2} \cup P_{4} \cup P_{5}}\left(P_{1}, P_{3}\right)$. We have

$$
\begin{aligned}
\lambda_{M / P_{2} \cup P_{4} \cup P_{5}}\left(P_{1}, P_{3}\right)= & r_{M / P_{2} \cup P_{4} \cup P_{5}}\left(P_{1}\right)+r_{M / P_{2} \cup P_{4} \cup P_{5}}\left(P_{3}\right)-r\left(M /\left\{P_{2} \cup P_{4} \cup P_{5}\right\}\right) \\
= & r\left(P_{1} \cup P_{2} \cup P_{4}\right)+r\left(P_{2} \cup P_{3} \cup P_{4}\right)-r(M)-r\left(P_{2} \cup P_{4}\right) \\
= & r(M)+k-r\left(P_{3}\right)+r(M)+k-r\left(P_{1}\right)-r(M)-r\left(P_{2} \cup P_{4}\right) \\
= & r(M)+2 k-r\left(P_{1}\right)-r\left(P_{3}\right)-r\left(P_{2}\right)-r\left(P_{4}\right)+\sqcap\left(P_{1}, P_{3}\right) \\
= & r(M)+2 k-r\left(P_{1} \cup P_{2}\right)-r\left(P_{3} \cup P_{4}\right) \\
& -\sqcap\left(P_{1}, P_{2}\right)-\sqcap\left(P_{3}, P_{4}\right)+\sqcap\left(P_{1}, P_{3}\right) \\
= & k-2 \sqcap\left(P_{1}, P_{2}\right)+\sqcap\left(P_{1}, P_{3}\right)
\end{aligned}
$$

where (3) follows from (2) by definition of connectivity function and noting that the connectivity of a single petal is $k$, (4) follows from (3) since $\sqcap\left(P_{1}, P_{2}\right)=\sqcap\left(P_{2}, P_{4}\right)$, and (6) follows from (5) by definition of the connectivity function, definition of flower and the hypotheses of the claim. Thus $\Pi^{*}\left(P_{1}, P_{3}\right)=$ $k-2 \sqcap\left(P_{1}, P_{2}\right)+\sqcap\left(P_{1}, P_{3}\right)$. The result follows by noting that $k-\sqcap\left(P_{1}, P_{2}\right)=$ $\Pi^{*}\left(P_{1}, P_{2}\right)$.

If $F=\left(P_{1}, P_{2}, P_{3}, P_{4} \ldots, P_{n}\right)$ is a $(k+1)$-flower then so is $F^{\prime}=\left(P_{1}^{\prime}, P_{2}^{\prime}, P_{3}^{\prime}, P_{4}^{\prime}\right)=$ $\left(P_{1}, P_{2}, P_{3}, P_{4} \cup \ldots \cup P_{n}\right)$ and $\sqcap_{F}\left(P_{i}, P_{j}\right)=\sqcap_{F^{\prime}}\left(P_{i}^{\prime}, P_{j}^{\prime}\right)$ for $i, j \in 1, \ldots, 4$. By the claim $\sqcap_{F^{\prime}}\left(P_{1}, P_{2}\right)-\sqcap_{F^{\prime}}\left(P_{1}, P_{3}\right)=\sqcap_{F^{\prime}}^{*}\left(P_{1}, P_{3}\right)-\sqcap_{F^{\prime}}^{*}\left(P_{1}, P_{2}\right)$ and so $\sqcap_{F}\left(P_{1}, P_{2}\right)-$ $\sqcap_{F}\left(P_{1}, P_{3}\right)=\sqcap_{F}^{*}\left(P_{1}, P_{3}\right)-\sqcap_{F}^{*}\left(P_{1}, P_{2}\right)$. Therefore $\xi=\sigma$.

The following lemma can be found in [13].

Lemma 3.0.7. Let $X$ and $Y$ be disjoint subsets of the groundset of a matroid $M$. Then $\sqcap(X, Y)+\Pi^{*}(X, Y)=\lambda(X)+\lambda(Y)-\lambda(X \cup Y)$.

Lemma 3.0.8. Let $F$ be a $(k+1)$-flower with at least 5 petals described by $(\mu, v, \xi)$, then $\mu+v+2 \xi=k$.

Proof. By Lemma 3.0.7 we see that $\sqcap\left(P_{1}, P_{2}\right)+\Pi^{*}\left(P_{1}, P_{2}\right)=k$. The result follows from this and the previous lemma.

There are two types of 2-flowers, a (1,0,0)-flower and a (0,1,0)-flower. This can easily be seen by the lemmas above but also follows from [5].

We now list the different types of 3-flowers. 
Definition 3.0.9. Suppose $F=P_{1}, \ldots, P_{n}$, where $n \geq 5$, is a 3 -flower.

i) If $F$ is a $(2,0,0)$-flower then $F$ is a paddle.

ii) If $F$ is a $(0,2,0)$-flower then $F$ is a copaddle.

iii) If $F$ is a $(1,1,0)$-flower then $F$ is spike-like.

iv) If $F$ is a $(0,0,1)$-flower then $F$ is swirl-like.

Clearly any 3-flowers with at least 5 petals is either a paddle, copaddle, spike-like or swirl-like.

Definition 3.0.10. A maximal flower, $F$, of a matroid, $M$, is a flower of $M$ such that if $F=\left(P_{1}, \ldots, P_{n}\right)$ then there is no $F^{\prime}=\left(P_{1}, \ldots, P_{i-1}, P_{i}^{\prime}, P_{i}^{\prime \prime}, P_{i+1}, \ldots, P_{n}\right)$ that is a flower of $M$ when $P_{i}^{\prime} \cup P_{i}^{\prime \prime}=P_{i}$. A maximal paddle (copaddle, spike-like flower, swirl-like flower) is a maximal flower that is a paddle (copaddle, spike-like flower, swirl-like flower respectively).

\subsection{Paddles}

Paddles are important because one of the structures we want to bridge is $M\left(K_{3, n}\right)$ which is a paddle. When we bridge $M\left(K_{3, n}\right)$, we are able reduce this to blocking a paddle. This means that general binary paddles are useful objects in this thesis. We therefore take a detour here to look at paddles.

Definition 3.1.1. If $F$ is a paddle in $M$ and $P$ is a petal of $F$ then $P$ is proper in $M$ if $P \notin \operatorname{cl}(E(M)-P)$. If $P \in \operatorname{cl}\left(P_{i}\right)$ for all $P_{i} \in F-P$ then $P$ is a guts petal.

Lemma 3.1.2. Let $F$ be a 3-flower of a binary matroid $M$ that has a maximal paddle with $n$ proper petals. Then $M$ has a minor $M^{\prime}$ that has a maximal paddle, $F=\left(P_{1}, \ldots, P_{n}, G\right)$, that can be represented by the following matrix:

$$
A=\left(\begin{array}{ccccc}
P_{1}^{\prime} & 0 & 0 & \ldots & 0 \\
0 & P_{2}^{\prime} & 0 & \ldots & 0 \\
0 & 0 & P_{3}^{\prime} & \ldots & 0 \\
\vdots & \vdots & \vdots & \ddots & \vdots \\
0 & 0 & 0 & \ldots & P_{n}^{\prime} \\
G_{1} & G_{2} & G_{3} & \ldots & G_{n}
\end{array}\right)
$$


where each $G_{i}$ contains at least one non-zero entry in every row and the $G_{i}^{\prime}$ s have 2 rows. Moreover, a petal, $P_{i}$, of $F$ contains the elements labelling the columns of $P_{i}^{\prime}$ and the rows labelling $P_{i}^{\prime}$, and $G$ contains the elements labelling the rows of the submatrices $G_{1}, \ldots, G_{n}$.

Proof. The matroid $M$ has a paddle $P=\left(P_{1}, \ldots, P_{n}, G\right)$ with $P_{1}, \ldots, P_{n}$ proper petals and $G$ a guts petal. As $P$ is a paddle $\sqcap\left(P_{i}, P_{j}\right)=2$ which means that there must be at least two basis element in the span of $P_{i}$ and $P_{j}$. This means that, for $i \in\{1, \ldots, n\}$ the submatrix $G_{i}$ contains at least two rows and for $j \neq i$ the submatrices $G_{i}$ and $G_{j}$ must contain at least two rows where both $G_{i}$ and $G_{j}$ contain a 1 . Consider $P_{i}, P_{j}$ and $P_{k}$. We know both $P_{j}$ and $P_{k}$ each must contain 2 rows where there are non zero entries in both the columns marked by $P_{j}, P_{k}$ and the columns marked by $P_{i}$. Suppose that these rows are not the same for both $P_{j}$ and $P_{k}$. This means that $\lambda\left(P_{i}\right)>2$, a contradiction.

Lemma 3.1.3. Every 3-connected binary matroid $M$ that has a paddle partition with at least $n \geq 5$ proper petals has an $M\left(K_{3, n}\right)$-minor.

Proof. This follows from Lemma 2.1.16.

\subsection{Pseudo-flowers}

Pseudo-flowers are a generalisation of flowers. The results in this section will almost certainly generalise to $k$-separations for any $k \geq 3$, but for this section we restrict out attention to the case when separations have order 1 or 2 .

Recall that $\kappa_{M}(X, Y)=\min \left\{\lambda_{M}(S): X \subseteq S \subseteq E(M)-Y\right\}$.

Definition 3.2.1. Let $M$ be a matroid. A pseudo-flower is an ordered partition $P_{1}, P_{2}, \ldots, P_{n}$ of $E(M)$ such that:

i) for any consecutive subset $P_{i}, \ldots, P_{j}$, we have $\lambda\left(P_{i} \cup \cdots \cup P_{j}\right) \in\{1,2\}$, and

ii) $\kappa\left(P_{i} \cup \cdots \cup P_{j}, P_{k} \cup \cdots \cup P_{l}\right)=\min \left\{\lambda\left(P_{i} \cup \cdots \cup P_{j}\right), \lambda\left(P_{k} \cup \cdots \cup P_{l}\right)\right\}$ for $i, j, k, l \in\{1, \ldots, n\}$ such that $\left[P_{i}, P_{j}, P_{k}, P_{l}\right]_{i}$.

The elements of $F$ are called petals. 
A pseudo-flower $F=\left(P_{1}, \ldots, P_{n}\right)$ is maximal if there is no $P_{i} \in\left(P_{1}, \ldots, P_{n}\right)$ such that $\left(P_{1}, \ldots, P_{i-1}, P_{i}^{\prime}, P_{i}^{\prime \prime}, P_{i+1}, \ldots, P_{n}\right)$ is a pseudo-flower. A petal $P_{i}$ in a pseudo-flower $F=\left(P_{1}, \ldots, P_{n}\right)$ is minimal if there is no partition of $P_{i}$ into $P_{i}^{\prime}$ and $P_{i}^{\prime \prime}$ such that $\left(P_{1}, \ldots, P_{i-1}, P_{i}^{\prime}, P_{i}^{\prime \prime}, P_{i+1}, \ldots, P_{n}\right)$ is a pseudo-flower. A consecutive subset of petals of $F$ is a subset, $S=\left\{P_{i}, \ldots, P_{j}\right\}$ where $\left[P_{1}, P_{i}, P_{j}\right]_{P-j}$, of petals of $F$ such that if $P_{k} \in S$ and $k \neq i, j$, then $P_{k+1}$ and $P_{k-1}$ are in $S$, and both $P_{i+1}$ and $P_{j-1}$ are in $S$.

Lemma 3.2.2. Let $\left(P_{1}, \ldots, P_{n}\right)$ be a pseudo-flower of a matroid $M$. If $\lambda\left(P_{i} \cup \cdots \cup\right.$ $\left.P_{j}\right)=1$, then either the connectivity of the union of any consecutive subset of $\left(P_{i}, \ldots, P_{j}\right)$ is 1 , or the connectivity of the union of any consecutive subset of the complement, $\left(P_{j+1}, \ldots, P_{i-1}\right)$, is 1 .

Proof. Suppose the theorem does not hold and consider a consecutive subset $P_{a}, \ldots, P_{b}$ of $\left(P_{i}, \ldots, P_{j}\right)$ and a consecutive subset $\left(P_{c}, \ldots, P_{d}\right)$ of $P_{j+1}, \ldots, P_{i-1}$. Suppose $\lambda\left(P_{a} \cup \cdots \cup P_{b}\right)=2=\lambda\left(P_{c} \cup \cdots \cup P_{d}\right)$. Then, since $\left(P_{1}, \ldots, P_{n}\right)$ is a pseudo-flower, $\kappa\left(P_{a} \cup \cdots \cup P_{b}, P_{c} \cup \cdots \cup P_{d}\right)=2$. However $P_{a} \cup \cdots \cup P_{b} \subseteq$ $P_{i} \cup \cdots \cup P_{j} \subseteq\left(E(M)-\left(P_{j+1} \cup \cdots \cup P_{i-1}\right)\right)$, and $\lambda\left(P_{i} \cup \cdots \cup P_{j}\right)=1$. By definition of $\kappa$ it follows that $\kappa\left(P_{a} \cup \cdots \cup P_{b}, P_{c} \cup \cdots \cup P_{d}\right)=1$, a contradiction.

Definition 3.2.3. A displayed 3-separation in pseudo-flower $F$ is a partition of the petals of $F$ into sets $A$ and $B$ such that $\lambda(\cup A)=2$.

Definition 3.2.4. Let $P_{i}$ be a petal of pseudo-flower $F$ of $M$. If $\lambda\left(P_{i}\right)=2$, then we call $P_{i}$ a 3-petal. If $\lambda\left(P_{i}\right)=1$ then we call $P_{i}$ a 2-petal.

Definition 3.2.5. Let $F=\left(P_{1}, \ldots, P_{n}\right)$ be a pseudo-flower of a matroid $M$.

1. A union of the elements of a set, $S$, of petals of $F$ is a concatenation of $S$ if the following holds. If $P_{i}$ and $P_{j}$ are in $S$ and $[i, j]_{1}$ then for all $k$ such that $[i, k, j]_{i}$ or $[j, k, i]_{j}$, the petal $P_{k}$ is in $S$.

A concatenation of $F$ is a collection of concatenations of disjoint subsets $S_{1}, \ldots, S_{m}$ of petals of $F$ so that every petal in $F$ is contained in some $S_{i}$ for $i \in\{1, \ldots, m\}$.

2. A concatenation of $F,\left(Q_{1}, \ldots, Q_{m}\right)$, is a flower concatenation of $F$ in $M$ if $\left(Q_{1}, \ldots, Q_{m}\right)$ is a flower in $M$. 
3. We say that a pseudo-flower is swirl-like if there is some concatenation of $\left(P_{1}, \ldots, P_{n}\right)$ that is a swirl-like flower; is a paddle if there is some concatenation of $\left(P_{1}, \ldots, P_{n}\right)$ that is a paddle; is a copaddle if there is some concatenation of $P_{1}, \ldots, P_{n}$ that is a copaddle; and is spike-like if there is some concatenation of $\left(P_{1}, \ldots, P_{n}\right)$ that is a spike-like flower.

4. The order of a pseudo-flower $F$ is the number of petals in a flower concatenation of $F$ with a maximal number of petals.

Note that the order defined above is different to the order in a flower as defined in [14] and [1].

The next lemma follows immediately from the definitions.

Lemma 3.2.6. If $F$ is a pseudo-flower then any concatenation of petals of $F$ is a pseudo-flower.

Lemma 3.2.7. Let $F$ be a pseudo-flower of a matroid $M$, and let $F^{\prime}$ be a flower concatenation of $F$ with at least five petals.

i) If $F^{\prime}$ is swirl-like then any flower concatenation of $F$ with at least five petals is swirl-like.

ii) If $F^{\prime}$ is spike-like then any flower concatenation of $F$ with at least five petals is spike-like.

iii) If $F^{\prime}$ is a paddle then any flower concatenation of $F$ with at least five petals is a paddle.

iv) If $F^{\prime}$ is copaddle then any flower concatenation of $F$ with at least five petals is a copaddle.

Proof. Let $\left(P_{a}^{\prime}, \ldots P_{m}^{\prime}\right)$ be the flower concatenation $F^{\prime}$ of $F$ in $M$, where $P_{k}^{\prime}=P_{1} \cup$ $\cdots \cup P_{k}-\left(\cup_{i<k} P_{i}^{\prime}\right.$ for $k \in\{1, \ldots, m\}$. Clearly a concatenation of $F^{\prime}$ with at least five petals is a flower of the same type as $F^{\prime}$. Consider splitting the petals of $F^{\prime}$ to give a new flower concatenation of $F$ in $M$. Consider two consecutive petals $P_{i}^{\prime}$ and $P_{j}^{\prime}$ of $F^{\prime}$. Let $\left(P_{1}, \ldots, P_{i}\right)$ be the petals of $F$ that make up $P_{i}^{\prime}$ and $\left(P_{i+1}, \ldots, P_{j}\right)$ be the petals of $F$ that make up $P_{j}^{\prime}$. Consider $\left(P_{1}^{\prime}, \ldots, P_{i}^{\prime}, P_{i+1}, \ldots, P_{k}\right)$ for some $k<j$. If this is a flower it is of the same type as $F$ since $\sqcap\left(P_{a}^{\prime}, P_{b}^{\prime}\right)$ is the same in both flowers if $\{a, b\} \nsubseteq\{i, j\}$. 


\section{Blocking and Separations in Flowers and Pseudo-Flowers}

Definition 3.2.8. Let $M$ be a matroid with pseudo-flower $F$. A displayed 3separation of $M$ by $F$ is a 3-separation $(A, B)$ of $M$ such that $A$ is a union of petals of $F$ and $B$ is a union of petals of $F$. We say that a flower $F$ is blocked in $M$ by $X$ if $M+X$ has no displayed 3-separations. We say that a petal $P_{i}$ of $F$ is blocked by an element $x \in X$ if $P_{i}$ is not 3-separating in $M+x$.

When it is clear from context that we are considering a pseudo-flower $F$ as a pseudo-flower of $M$ we may talk about an element $x$ blocking a petal $P$ of $F$ to mean $x$ blocks $P$ in $M$. We may also use a similar abbreviation for sets of elements.

\subsection{Swirl-like Pseudo-flowers}

We look at swirl-like pseudo-flowers in some detail here since we believe that the problem of bridging a wheel reduces to the problem of blocking a swirl-like pseudo-flower. Throughout this section we work under the following hypotheses.

- $M$ is a binary matroid, and

- $F=\left(P_{1}, \ldots, P_{n}\right)$ is a swirl-like pseudo-flower of $M$ of order at least five.

Recall that if $M$ is a matroid and $A \subseteq E(M)$ we use $\langle A\rangle$ to denote the elements from the ambient extended projective space that are in the span of the elements of A.

\section{Definition 3.3.1.}

i) A clump in $F$ is a consecutive subset $\left(P_{i}, P_{i+1}, \ldots, P_{j}\right)$ of petals such that the following holds. For all $i$ such that $\left[i, i^{\prime}, j^{\prime}, j\right]_{i}$ we have $\lambda\left(P_{i^{\prime}} \cup \cdots \cup P_{j^{\prime}}\right)=1$.

ii) A clump is maximal if it is maximal with respect to this property.

iii) A concatenation of a consecutive set, $S=\left\{P_{i}, \ldots, P_{j}\right\}$, of petals is weak if $\left(P_{i}, \ldots, P_{j}\right)$ is a clump.

The following lemma is trivial. 
Lemma 3.3.2. If $\left(P_{1}, \ldots, P_{i}\right)$ is a clump in $F$ with at least two proper petals then $\left(P_{1}, \ldots, P_{i}, P_{i+1} \cup \cdots \cup P_{n}\right)$ is a 2 -flower.

Recall that there are two types of 2-flower, a (1,0,0)-flower or a $(0,1,0)$-flower. The next lemma shows that the petals on either side of a clump "see" this clump in the same way.

Lemma 3.3.3. Let $F=\left(Q_{1}, C, Q_{2}, Q_{3}, Q_{4}, Q_{5}\right)$ be a swirl-like pseudo-flower such that $\left(Q_{1} \cup C, Q_{2}, Q_{3}, Q_{4}, Q_{5}\right)$ is a flower concatenation of $F$ in $M$, where $C$ is a clump and $\lambda\left(Q_{i}\right)=2$ for $i \in\{1,2,3,4,5\}$. Then either:

i) $\sqcap\left(Q_{1}, C\right)=\sqcap\left(Q_{2}, C\right)=1$, or

ii) $\sqcap\left(Q_{1}, C\right)=\sqcap\left(Q_{2}, C\right)=0$.

Proof. Since $\lambda(C)=1$ it follows that $\sqcap\left(Q_{i}, C\right) \leq 1$ for $i \in\{1,2\}$. Suppose $\sqcap\left(Q_{1}, C\right)=1$. By Lemma 3.2.7 $\left(Q_{1} \cup C, Q_{2}, Q_{3}, Q_{4}, Q_{5}\right)$ is a swirl-like flower, and hence $\sqcap\left(Q_{1} \cup C, Q_{2}\right)=1$. Therefore,

$$
\begin{aligned}
r\left(Q_{1} \cup C \cup Q_{2}\right) & =r\left(Q_{1} \cup C\right)+r\left(Q_{2}\right)-1 \\
& =r\left(Q_{1}\right)+r(C)+r\left(Q_{2}\right)-2 .
\end{aligned}
$$

Suppose $\sqcap\left(Q_{2}, C\right)=0$. Then

$$
\begin{aligned}
r\left(Q_{1} \cup C \cup Q_{2}\right) & =r\left(Q_{1}\right)+r\left(Q_{2} \cup C\right)-1 \\
& =r\left(Q_{1}\right)+r(C)+r\left(Q_{2}\right)-1 .
\end{aligned}
$$

Together these equations give a contradiction.

Definition 3.3.4. Let $F=\left(P_{1}, \ldots, P_{n}\right)$ be a swirl-like pseudo-flower of a matroid $M$. Let $C=\left(P_{i}, \ldots, P_{j}\right)$ be a clump of $F$ in $M$. We say that $C$ is joint-based if for some concatenation $\left(Q_{1}, C, Q_{2}, Q_{3}, Q_{4}, Q_{5}\right)$ of $F$ with $\lambda\left(Q_{i}\right)=2$ for $i \in$ $\{1,2,3,4,5\}$, we have $\sqcap\left(Q_{1}, C\right)=1$. We say that $C$ is rim-based if for some concatenation $\left(Q_{1}, C, Q_{2}, Q_{3}, Q_{4}, Q_{5}\right)$ of $F$ with $\lambda\left(Q_{i}\right)=2$ for $i \in\{1,2,3,4,5\}$, we have $\sqcap\left(Q_{1}, C\right)=0$.

We show shortly that a clump will either be joint-based or rim-based (and not be both). 
Lemma 3.3.5. Let $F$ be a swirl-like pseudo-flower of $M$ and $C$ be a clump of $F$. There are no clumps $A, B$ that are subsets of $C$ and are such that $A$ is joint-based and $B$ is rim-based.

Proof. Let $\left(Q_{1}, C, Q_{2}, Q_{3}, Q_{4}, Q_{5}\right)$ be a concatenation of $F$ with $\lambda\left(Q_{i}\right)=2$ for $i \in\{1,2,3,4,5\}$. Suppose there is a subset $A$ of $C$ that is a joint-based clump. Then there is some $e$ such that $e \in\left(\langle A\rangle \cap\left\langle Q_{1}\right\rangle\right)$. Suppose there is some clump $B$ contained in $C$ that is rim-based, there is some $f \in\left(\langle B\rangle-\left\langle Q_{1}\right\rangle\right)$ with the property that $f \in\left\langle E(M)-\cup_{i \in B} P_{i}\right\rangle$. Since $e$ and $f$ are not equal or parallel then $\lambda(C) \geq 2$, a contradiction.

This means that if a clump $C=P_{i}, \ldots, P_{k}$ is joint-based (respectively rim-based) then any subset of $C$ is also joint-based (respectively rim-based).

The next lemma shows that different concatenations "see" a clump in the same way.

Lemma 3.3.6. Let $F$ be a swirl-like pseudo-flower of a matroid $M$. Let $\left(Q_{1}, C, Q_{2}, Q_{3}, Q_{4}, Q_{5}\right)$ and $\left(Q_{1}^{\prime}, C, Q_{2}^{\prime}, Q_{3}^{\prime}, Q_{4}^{\prime}, Q_{5}^{\prime}\right)$ be concatenations of $F$ where $C$ is weak and $\lambda\left(Q_{i}\right)=\lambda\left(Q_{i}^{\prime}\right)=2$ for $i \in\{1,2,3,4,5\}$. Then for $j \in\{0,1\}$ we have $\sqcap\left(Q_{1}, C\right)=\sqcap\left(Q_{2}, C\right)=j$ if, and only if, $\sqcap\left(Q_{1}^{\prime}, C\right)=\sqcap\left(Q_{2}^{\prime}, C\right)=j$.

It is clear that if $P_{1}, \ldots, P_{k}$ is a clump then $\left(P_{1}, . ., P_{k}, P_{k+1} \cup \cdots \cup P_{n}\right)$ is a $(1,0,0)$ flower or a $(0,1,0)$-flower. The next lemma follows immediately from this.

Lemma 3.3.7. Let $M$ be a matroid with swirl-like pseudo-flower F. Suppose $C=\left(P_{1}, \ldots, P_{j}\right)$ is a maximal clump of $F$ in $M$ containing at least two petals. If $\left(P_{1}, \ldots, P_{j}\right)$ is joint-based then $\left(P_{1}, . ., P_{j}, P_{j+1} \cup \cdots \cup P_{n}\right)$ is a $(1,0,0)$-flower, and if $\left(P_{1}, \ldots, P_{j}\right)$ is rim-based then $\left(P_{1}, . ., P_{j}, P_{j+1} \cup \cdots \cup P_{n}\right)$ is a $(0,1,0)$-flower.

The following lemma then follows easily.

Lemma 3.3.8. Let $\left(P_{1}, \ldots, P_{n}\right)$ be a swirl-like pseudo-flower of a matroid $M$. If there is a consecutive set of petals $S=\left(P_{1}, \ldots, P_{t}\right)$ such that $S$ is a clump, then $\left(P_{1}^{\prime}, \ldots, P_{t}^{\prime}, P_{t+1} \cup \ldots \cup P_{n}\right)$ is a swirl-like pseudo-flower of $M$ where all members of $\left\{P_{1}^{\prime}, \ldots, P_{t}^{\prime}\right\}$ are distinct and $P_{i}^{\prime} \in\left\{P_{1}, \ldots, P_{t}\right\}$ for $i \in\{1, \ldots, t\}$.

This tells us that the petals in a clump can be reordered and we still have a swirllike pseudo-flower. 
Definition 3.3.9. Let $\left(P_{1}, \ldots, P_{n}\right)$ be a swirl-like pseudo-flower of a matroid $M$. A concatenation $Q$ of petals $S$ is strong if $\lambda(Q)=2$ and whenever $\left\{P_{1}, \ldots, P_{i}\right\}$ is a maximal clump either $\left\{P_{1} \cup \cdots \cup P_{i}\right\} \subseteq Q$ or $\left\{P_{1} \cup \cdots \cup P_{i}\right\} \cap Q=\emptyset$. Let $\left(Q_{1}, Q_{2}, Q_{3}, Q_{4}, Q_{5}\right)$ be a concatenation of $F$. Then $\left\{Q_{1}, Q_{2}\right\}$ is a strong pair if both $Q_{1}$ and $Q_{2}$ are strong and $\lambda\left(Q_{3} \cup Q_{4} \cup Q_{5}\right)=2$.

If $\left\{Q_{1}, Q_{2}\right\}$ is a strong pair, then there is be some element in $\left\langle Q_{1}\right\rangle \cap\left\langle Q_{2}\right\rangle$. We say that two strong pairs are equivalent if this element is the same for both pairs. We formalise this below.

Definition 3.3.10. Suppose $\left(Q_{1}, Q_{2}\right)$ and $\left(Q_{1}^{\prime}, Q_{2}^{\prime}\right)$ are two strong pairs of $F$ in $M$. We say $\left(Q_{1}, Q_{2}\right) \sim\left(Q_{1}^{\prime}, Q_{2}^{\prime}\right)$ if $\left\langle Q_{1}\right\rangle \cap\left\langle Q_{2}\right\rangle=\left\langle Q_{1}^{\prime}\right\rangle \cap\left\langle Q_{2}^{\prime}\right\rangle$.

Observe that " " is an equivalence relation.

The next lemma shows that we can shift (some) maximal clumps into and out of concatenations in strong pairs.

Lemma 3.3.11. Suppose $\left(Q_{1}, Q_{2}\right)$ and $\left(Q_{1}^{\prime}, Q_{2}^{\prime}\right)$ are strong pairs in $F$.

i) If $Q_{1} \subseteq Q_{1}^{\prime}$ and $Q_{2}=Q_{2}^{\prime}$ then $\left(Q_{1}, Q_{2}\right) \sim\left(Q_{1}^{\prime}, Q_{2}^{\prime}\right)$.

ii) If there is some $C=P_{i} \cup \cdots \cup P_{j}$ where $\left(P_{i}, \ldots, P_{j}\right)$ is a maximal joint-based clump, and $\left(Q_{1}, Q_{2}\right)=\left(Q_{1}^{\prime}-C, Q_{2}^{\prime} \cup C\right)$, then $\left(Q_{1}, Q_{2}\right) \sim\left(Q_{1}^{\prime}, Q_{2}^{\prime}\right)$.

Proof. i) is clear. For ii) suppose $\left(Q_{1}, Q_{2}\right)=\left(Q_{1}^{\prime}-C, Q_{2}^{\prime} \cup C\right)$. Consider $a \in$ $\left\langle Q_{1}\right\rangle \cap\left\langle Q_{2}\right\rangle$. This means that $a \in\left\langle Q_{1}^{\prime}-C\right\rangle$ and $a \in\left\langle Q_{2}^{\prime} \cup C\right\rangle$. Assume $a \in\left\langle Q_{1}^{\prime}\right\rangle$ and $a \notin\left\langle Q_{2}^{\prime}\right\rangle$. There is some $b \in\left\langle Q_{2}^{\prime}\right\rangle \cap\langle C\rangle$ and if $b \neq a$ then $\lambda(C) \geq 2$ contradicting the fact that $C$ is a clump. This shows that $\left\langle Q_{1}\right\rangle \cap\left\langle Q_{2}\right\rangle \subseteq\left\langle Q_{1}^{\prime}\right\rangle \cap\left\langle Q_{2}^{\prime}\right\rangle$. A similar argument shows that $\left\langle Q_{1}^{\prime}\right\rangle \cap\left\langle Q_{2}^{\prime}\right\rangle \subseteq\left\langle Q_{1}\right\rangle \cap\left\langle Q_{2}\right\rangle$

A way of visualising a swirl-like pseudo-flower is given below: 


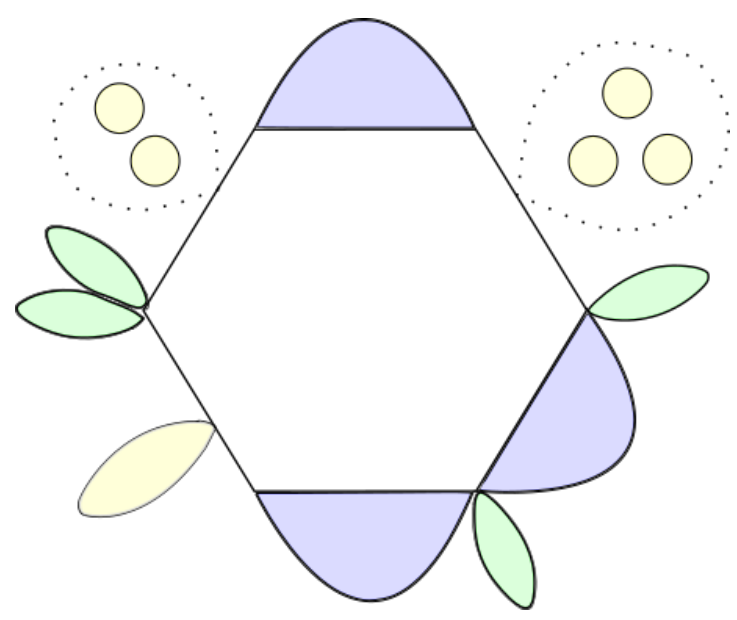

where the purple areas represent 3-petals, yellow areas represent rim-based 2petals and green areas represent joint-based 2-petals. We want to be able to add a point everywhere the span of two petals intersects. We call these elements "joints", and they turn out to be rather annoying to define.

Definition 3.3.12. Consider the set $S$ of equivalence classes of the strong pairs of $F$. Let $T$ be a set containing one strong pair from each part of $S$. Let the elements of $T$ be $\left(Q_{1}, R_{1}\right),\left(Q_{2}, R_{2}\right), \ldots,\left(Q_{k}, R_{k}\right)$. For each $i \in\{1, \ldots, k\}$ we define $j_{i}$ to be an element of $\left\langle Q_{i}\right\rangle \cap\left\langle R_{i}\right\rangle$ that is not in $E(M)$. We say that $j_{i}$ is a joint of $F$ and that $J_{F}=\left\{j_{1}, \ldots, j_{k}\right\}$ is the set of joints of $F$.

From now on we use $M^{+}$to refer to the matroid obtained by extending $M$ by the set of joints of $M$.

We define a partition $F^{+}=\left(Q_{1}, \ldots, Q_{m}\right)$ of $E\left(M^{+}\right)$so that every for every $P_{i}$ in $F$ the petal $P_{1} \in F^{+}$and every element $j$ of $E\left(M^{+}\right)-E(M)$ is in an equivalence class that no element of $E\left(M^{+}\right)-j$. In other words, the classes in the partition of $M^{+}$ are the classes in the partition $F$ of $M$ along with one class for each element of $J$. The pseudo-flower $F$ is swirl-like so has a natural ordering on the petals (that is on the equivalence classes of $F$ ). We introduce an ordering on the equivalence classes of $F^{+}$. If $\left(Q_{i}, R_{i}\right)$ is a strong pair in $F$ and $\left(Q_{i}, R_{i}\right)=\left(P_{1} \cup \cdots \cup P_{b}, P_{b+1} \cup \cdots \cup P_{c}\right)$ then in $F^{+}$let the ordering of the equivalence classes be $\left[P_{b}, j_{i}, P_{b+1}\right]_{P_{b}}$ where there is no $P_{c}$ such that $\left[P_{b}, P_{c}, j_{i}\right]_{P_{b}}$ or $\left[j_{i}, P_{c}, P_{b+1}\right]_{P_{b}}$. After possible relabelling we may assume that the joints appear in consecutive order in the same direction as the petals of $F$.

Observe the following. 
Lemma 3.3.13. If $\left(Q_{i}, R_{i}\right)$ and $\left(Q_{i}^{\prime}, R_{i}^{\prime}\right)$ are strong pairs in $F$ and $\left(Q_{i}^{\prime}, R_{i}^{\prime}\right) \sim$ $\left(Q_{i}, R_{i}\right)$, then $j_{i} \in \operatorname{cl}\left(Q_{i}^{\prime}\right) \cap \operatorname{cl}\left(R_{i}^{\prime}\right)$.

We want to show that $F^{+}$is a swirl-like pseudo-flower but first we need a couple of lemmas. These can be found in [10].

Lemma 3.3.14. Let $P$ and $Q$ be sets in a matroid $N$ with $\lambda(P)=\lambda(Q)=\lambda(P \cap$ $Q)=\lambda(P \cup Q)=t$. Then $P$ and $Q$ are a modular pair.

As a corollary of this we get

Corollary 3.3.15. Suppose $P$ and $Q$ are petals in a swirl-like pseudo-flower and $\lambda(P)=\lambda(Q)=\lambda(P \cap Q)=\lambda(P \cup Q)=2$. Then $P$ and $Q$ are a modular pair.

Lemma 3.3.16. Let $N$ be a matroid and $z \in E(N)$. Let $X$ and $Y$ be a modular pair in the matroid $N \backslash z$. If $z \in \operatorname{cl}(X)$ and $z \in \operatorname{cl}(Y)$, then $z \in \operatorname{cl}(X \cap Y)$.

Lemma 3.3.17. The partition $\mathrm{F}^{+}$of $\mathrm{M}^{+}$is a pseudo-flower.

Proof. Suppose $F$ has $n$ joints and for $i \in\{1, \ldots, n\}$ let $M_{i}=M^{+} \mid((E(M) \cup$ $\left.\left\{j_{1}, \ldots, j_{i}\right\}\right)$ for joints $j_{1}, \ldots, j_{i}$ of $F$. Let $F_{i}$ be the partition of $E\left(M_{i}\right)$ obtained by restricting $F^{+}$to $E\left(M_{i}\right)$. The result follows immediately from the following claim.

Claim 3.3.18. For $i \in\{1, \ldots, n\} F_{i}$ is a swirl-like pseudo-flower of $M_{i}$ and $j_{i+1}, \ldots, j_{n}$ are joints of $F_{i}$.

Proof. We proceed by induction. For the base case notice that $F$ is a swirl-like pseudo-flower of $M$ and $\left\{j_{1}, \ldots, j_{n}\right\}$ are joints of $F$. Assume that $F_{i-1}$ is a swirllike pseudo-flower and that $\left\{j_{i}, \ldots, j_{n}\right\}$ are joints of $F_{i-1}$. Consider extending $F_{i-1}$ by $j_{i}$. This means that $F_{i}=\left(P_{1}, \ldots, P_{i},\left\{j_{i}\right\}, P_{i+1}, \ldots, P_{n}\right)$. Consider some concatenation $\left(P_{1}, \ldots, P_{a-1}, P_{a} \cup \cdots \cup P_{i} \cup\left\{j_{i}\right\} \cup P_{i+1} \cup \cdots \cup P_{b}, P_{b+1}, \ldots, P_{n}\right)=$ $\left(P_{1}, \ldots, P_{a-1}, Y \cup\left\{j_{i}\right\} \cup Z, P_{b+1}, \ldots, P_{n}\right)$ of $F_{i}$. To show that for any $a, b$ such that $[a, b]_{1}$ we have that $F_{i}$ is a swirl-like pseudo-flower, it is enough to show that $\lambda_{M_{i}}\left(Y \cup\left\{j_{i}\right\} \cup Z\right) \leq 2$.

Suppose $\lambda_{M_{i-1}}(Y)=2$, then $j_{i} \in \operatorname{cl}_{M_{i}}(Y \cup Z)$ and so $\lambda_{M_{i}}\left(Y \cup\left\{j_{i}\right\} \cup Z\right)=2$. The argument is similar when $\lambda_{M_{i-1}}(Z)=2$, so assume that $\lambda_{M_{i-1}}(Y) \leq 1$ and $\lambda_{M_{i-1}}(Z) \leq 1$. We may also assume that both $Y$ and $Z$ are non-empty as otherwise the result follows. Therefore we may assume that $\lambda_{M_{i-1}}(Y)=1$ and $\lambda_{M_{i-1}}(Z)=1$. 
By the definition of a joint we know that $Y$ and $Z$ are not subsets of the same clump. Therefore, $\lambda_{M_{i-1}}(Y \cup Z) \geq 2$.

Let $\left(Q_{1}, Q_{2}, Y \cup Z, Q_{3}\right)$ be a concatenation of $F_{i-1}$ with $\lambda\left(Q_{1}\right)=\lambda\left(Q_{2}\right)=\lambda\left(Q_{3}\right)=$ 2. We know that $j_{i} \in \operatorname{cl}_{M_{i}}\left(Q_{2} \cup Y \cup Z\right)$ and $j_{i} \in \mathrm{cl}_{M_{i}}\left(Q_{3} \cup Y \cup Z\right)$. By Corollary 3.3.15 $\left\{Q_{2} \cup Y \cup Z, Q_{3} \cup Y \cup Z\right\}$ is a modular pair in $M_{i-1}$. By Lemma 3.3.16 $j_{i} \in \operatorname{cl}_{M_{i}}(Y \cup Z)$. Thus $\lambda_{M_{i}}\left(Y \cup Z \cup\left\{j_{i}\right\}\right)=2$, and so $F_{i}$ is a swirl-like pseudoflower.

We must show that $j_{i+1}, \ldots, j_{n}$ are joints of $F_{i}$. Consider $k \in\{i+1, \ldots, n\}$. There is a strong pair $\left(P_{k}, Q_{k}\right)$ with $j_{k} \in \operatorname{cl}\left(P_{k}, Q_{k}\right)$. Let $P_{k}^{\prime}=\operatorname{cl}_{M_{i}}\left(P_{k}\right) \cap\left(P_{k} \cup\left\{j_{1}, \ldots, j_{i}\right\}\right)$ and $Q_{k}^{\prime}=\operatorname{cl}_{M_{i}}\left(Q_{k}\right) \cap\left(Q_{k} \cup\left\{j_{1}, \ldots, j_{i}\right\}\right)$. We see that $\left(P_{k}^{\prime}, Q_{k}^{\prime}\right)$ is a strong pair of $F_{i}$ and thus $j_{k}$ is a joint of $F_{i}$.

Lemma 3.3.19. There is a bijection between the set of joints of $F$ and the maximal joint-based clumps of $\mathrm{F}^{+}$. Moreover, each maximal joint-based clump of $\mathrm{F}^{+}$is either:

i) a joint of $F$, or

ii) the union of a joint of $F$ and a maximal jointt-based clump of $F$

Proof. Assume that $\left(C_{1}, \ldots, C_{j}\right)$ is a maximal joint-based clump of $F$ and let $C=$ $C_{1} \cup \cdots \cup C_{j}$. Then there is a concatenation $\left(Q_{1}, C, Q_{2}, Q_{3}\right)$ of $F$ such that $\lambda\left(Q_{i}\right)=$ 2 for $i \in\{1,2,3\}$ and $\sqcap\left(Q_{1}, Q_{2}\right)=\sqcap\left(Q_{1}, C \cup Q_{2}\right)=\sqcap\left(Q_{1} \cup C, Q_{2}\right)=1$. Moreover, $\left(Q_{1}, C \cup Q_{2}\right) \sim\left(Q_{1} \cup C, Q_{2}\right)$ so there is a joint $j$ of $F$ so that $j \in \operatorname{cl}_{M^{+}}\left(Q_{1}\right) \cap$ $\mathrm{cl}_{M^{+}}\left(Q_{2}\right)$. It follows that $\{j\} \cup\left\{C_{1}, \ldots, C_{j}\right\}$ is a maximal clump of $F^{+}$.

Now suppose that $j$ is a joint of $F$. Then $j$ is a member of a maximal joint-based clump of $F^{+}$. Either that clump is $\{j\}$ and there is no corresponding clump in $F$, or that clump is some collection of sets, $C$. Then $C-\{j\}$ is a clump in $F$.

Lemma 3.3.20. Let $S$ be a minimal set of petals such that the concatenation, $P$, of $S$ is a strong concatenation of petals of $F$ and $S$ is minimal with respect to this property, and let $\left(Q_{1}, P, Q_{2}, Q_{3}, Q_{4}\right)$ be a concatenation of $F$ such that $\lambda\left(Q_{1}\right)=$ $\lambda\left(Q_{2}\right)=\lambda\left(Q_{3}\right)=\lambda\left(Q_{4}\right)=2$. Then $\mathrm{cl}_{M^{+}}(P)$ contains exactly two joints, $j_{1}$ and $j_{2}$ where $j_{1} \in \operatorname{cl}\left(Q_{1}\right)$ and $j_{2} \in \operatorname{cl}\left(Q_{2}\right)$. 
Proof. Since $\left(Q_{1}, P\right)$ and $\left(P, Q_{2}\right)$ are both strong pairs there exist distinct joints $j_{1} \in \mathrm{cl}_{M^{+}}\left(Q_{1}\right) \cap \mathrm{cl}_{M^{+}}(P)$ and $j_{2} \in \mathrm{cl}_{M^{+}}\left(Q_{2}\right) \cap \mathrm{cl}_{M^{+}}(P)$. Suppose there were some other $j \in \mathrm{cl}_{M^{+}}(P)$. Then there would have to be a strong pair $(A, B)$ with $j$ in the closure of both sides and $(A, B) \nsim\left(Q_{1}, P\right)$ and $(A, B) \nsim\left(P, Q_{2}\right)$. This strong pair would have be such that one of $A, B$ contains some strict subset of $P$, which is a contradiction to the minimality of $P$.

It immediately follows that a 3-petal of a swirl-like pseudo-flower contains exactly two joints in its closure.

The following lemma is clear

Lemma 3.3.21. If $P$ is a joint-based 2-petal of $F$ then there is exactly one joint, $j$ such that $j \in\langle P\rangle$.

We also want the following lemma.

Lemma 3.3.22. If $P_{1}$ is a rim-based 2-petal of $F$ then there is a unique element in $\left\langle P_{1}\right\rangle \cap\left\langle\cup\left(F-P_{1}\right)\right\rangle$ and this element forms a triangle with exactly two joints of $F$.

Proof. Since $\lambda\left(P_{1}\right)=1$, there is a unique element $r$ such that $r \in\left\langle P_{1}\right\rangle \cap\langle\cup(F-$ $P)\rangle$. Let $F^{\prime}=\left(P_{1}, Q_{2}, \ldots, Q_{m}\right)$ be a concatenation with $m \geq 4$ and with the same set of joints as $F$ and such that $\lambda\left(Q_{2}\right)=\lambda\left(Q_{m}\right)=2$. Since $\sqcap\left(Q_{i}, P_{1}\right)=0$ for $i \in\{2, \ldots, n\}$ it follows that $r$ is not parallel to any joint of $F^{\prime}$ or $F$. Suppose that $r$ does not form a circuit with elements contained in $\left\langle Q_{m} \cup Q_{2}\right\rangle$. We know that $\lambda\left(Q_{n} \cup Q_{2} \cup r\right)=2$, so from this it follows that $\lambda\left(P_{1} \cup Q_{2}\right) \leq 2$ - a contradiction. Therefore $P_{1}$ is in a circuit with elements contained in $\left\langle Q_{m} \cup Q_{1}\right\rangle$. To see that these elements must be $j_{1}$ and $j_{m}$ - the joints of $Q_{m}$ and $Q_{1}$ - say that $r \in\left\langle Q_{m} \cup Q_{1}\right\rangle$ and $r \notin\left\langle\left\{j_{m}, j_{1}\right\}\right\rangle$, Since $\lambda\left(Q_{m}, P_{1}\right)=2$ we have $j_{0} \in\left\langle P_{1} \cup Q_{m}\right\rangle$. Similarly $j_{1} \in$ $\left\langle P_{1} \cup Q_{2}\right\rangle$. Since $\lambda\left(Q_{m} \cup P_{1}\right)=2$ the result follows.

Definition 3.3.23. If $P_{i}$ is a joint-based 2-petal or a 3 petal of $F$ then the joints of $P$, denoted $J(P)$, are the joints of $F$ that are in $\langle P\rangle \cap\langle\cup(F-P)\rangle$. If $P$ is a 3-petal then the rim element of $P$ is the unique element contained in $\langle J(P)\rangle$ and not in $E(M)$.

If $P$ is a rim-based 2-petal of $F$ then the rim element, $r$, of $P$ is the unique element in $\langle P\rangle \cap\langle\cup(F-P)\rangle$ that is not in $E(M)$. The joints of $P$ are the minimal set of joints of $F$ containing $r$ in their closure. 
If $P$ is a petal of $F$, then the basepoints of $P$, denoted $B(P)$ are the set of joint and rim-elements of $P$.

Throughout the remainder of this chapter we let $J$ denote the set of joints of $F$.

Lemma 3.3.24. $J$ is an independent set.

Proof. Consider $F^{+}$and let $j$ be a joint of $F$. Let $Q_{1}$ and $Q_{2}$ be to petals in a concatenation of $F^{+}$with the property that $\lambda\left(Q_{1}\right)=\lambda\left(Q_{2}\right)=2$ and $Q_{1}$ and $Q_{2}$ are minimal with respect to this property. Suppose that $F^{+}$displays $\left(Q_{1},\{j\}, Q_{2}, Q_{3}\right)$. Since $Q_{1}$ and $Q_{2}$ are minimal there is no joint in $\left\langle Q_{1} \cup Q_{2}\right\rangle-\left\langle E(M)-\left(Q_{1} \cup Q_{2}\right)\right\rangle$, so all members of $J-\{j\}$ are contained in $\mathrm{cl}_{M^{+}}\left(Q_{3}\right)$. It follows from elementary results about flowers (see, for example, [14]) that if $j \in \operatorname{cl}\left(Q_{3}\right)$ then $F^{+}$would be spike-like. Therefore, $j \notin \operatorname{cl}\left(Q_{3}\right)$, and so $j$ is not in the closure of $J-\{j\}$ and thus the set of joints of $F$ is an independent set.

Definition 3.3.25. A maximal swirl-like pseudo-flower of $M$ is a swirl-like pseudo-flower of $M$ in which no petals can be partitioned to give a swirl-like pseudo-flower of $M$ with more petals.

We want to show that if a swirl-like pseudo-flower is maximal in $M$ then it has no petal containing certain types of 2 -separations.

Lemma 3.3.26. Let $(A, B)$ be a 3-separation of $M$ such that $A=\left(P^{\prime} \cup P_{2} \cup \cdots \cup P_{k}\right)$ and $B=\left(P_{k+1} \cup \cdots \cup P_{n} \cup P^{\prime \prime}\right)$ where $P^{\prime} \cup P^{\prime \prime}=P_{1}$ and $P^{\prime} \neq \emptyset$ and $P^{\prime \prime} \neq \emptyset$. Suppose $\lambda\left(P_{2}, \ldots, P_{k}\right)$ and $\lambda\left(P_{k+1} \cup \cdots \cup P_{n}\right)=2$. Then $\left(P^{\prime}, P_{2}, \ldots, P_{n}, P^{\prime \prime}\right)$ is a swirl-like pseudo-flower.

Proof. Without loss of generality we may assume that $P^{\prime} \cup P_{2} \cup \cdots \cup P_{j} \subseteq P_{1} \cup$ $P_{2} \cup \cdots \cup P_{k}$ for $[1, j, k]_{1}$. Consider the sets $P_{1} \cup P_{2} \cup \cdots \cup P_{j}$ and $P^{\prime} \cup P_{2} \cup \cdots \cup P_{k}$. Since $\lambda\left(P_{k+1} \cup \cdots \cup P_{n}\right)=2$ it must be that $\left|P_{k+1} \cup \cdots \cup P_{n}\right| \geq 2$. Therefore, by uncrossing, $\lambda\left(P^{\prime} \cup P_{2} \cup \cdots \cup P_{j}\right) \leq 2$. The result follows easily for all other subsets

The proof of condition $i$ ) of the definition of swirl-like pseudo-flowers follows if we can show that $\lambda\left(P^{\prime} \cup P_{2} \cup \cdots \cup P_{j}\right)=1$ implies that either $\lambda\left(P^{\prime} \cup P_{2} \cup \cdots \cup P_{i}\right)=$ 1 or $\lambda\left(P_{l} \cup \cdots \cup P_{i}\right)=1$ for all $l, i$ such that $[1, l, i, j]_{1}$. By $\left.i i\right)$ of the definition of pseudo-flower condition $i i)$ holds on sets of the form $\left(P_{l} \cup \cdots \cup P_{i}\right)$. Now consider $\lambda\left(P_{1} \cup \cdots \cup P_{i}\right)$, and $\lambda\left(P^{\prime} \cup P_{2} \cup \cdots \cup P_{i}\right)$. By submodularity of the connectivity 
function $\lambda\left(P_{1} \cup \cdots \cup P_{i}\right)+\lambda\left(P^{\prime} \cup P_{2} \cup \cdots \cup P_{j}\right) \geq \lambda\left(P_{1} \cup P_{2} \cup \cdots \cup P_{i}\right)+\lambda\left(P^{\prime} \cup P_{2} \cup\right.$ $\left.\cdots \cup P_{i}\right)$. Since $F$ has at least four joints $\lambda\left(P_{1} \cup P_{2} \cup \cdots \cup P_{i}\right) \geq \lambda\left(P_{1} \cup \cdots \cup P_{j}\right)$. Therefore $\lambda\left(P^{\prime} \cup P_{2} \cup \cdots \cup P_{i}\right) \leq \lambda\left(P^{\prime} \cup P_{2} \cup \cdots \cup P_{j}\right)$.

Lemma 3.3.27. Suppose $F$ is a maximal swirl-like pseudo-flower. Then there is no petal $P_{i}$ of $F$ such that $M \mid P_{i}$ contains a 2-separation $(A, B)$ with the following property,

1. If $P_{i}$ is a joint-based 2-petal then the joint, $j_{1}$, of $P$ is in $\operatorname{cl}(A) \cap \operatorname{cl}(B)$.

2. If $P$ is a rim-based 2-petal or a 3-petal with joints $j_{1}$ and $j_{2}$, then $j_{1} \in \operatorname{cl}(A)$ and $j_{2} \in \operatorname{cl}(B)$.

Proof. Suppose $P_{i}$ has a single joint, $j_{1}$. Let $X_{1}$ and $X_{2}$ be two disjoint sets of petals of $F-P_{i}$ with the property that $X_{i}$ is a consecutive set and $X_{2}$ is a consecutive set. We show that if $j_{1} \in \operatorname{cl}\left(A \cup X_{1}\right)$ then $\lambda\left(A \cup X_{1}\right)=2$ and thus $F$ is not maximal. Without loss of generality let $j_{1} \in \operatorname{cl}\left(X_{1}\right)$

$$
\begin{aligned}
\lambda\left(A \cup X_{1}\right) & =r\left(A \cup X_{1}\right)+r\left(B \cup X_{2}\right)-r(M) \\
& =r(A)+r\left(X_{1}\right)-1+r(B)+r\left(X_{2}\right)-1-r(M) \\
& =r(P)+1+r\left(X_{1} \cup X_{2}\right)+1-2-r(M) \\
& =r(M)+2-r(M) \\
& =2
\end{aligned}
$$

Where (2) follows from (1) since $j_{1} \in \operatorname{cl}\left(A \cap X_{1}\right)$ and $j_{1} \in \operatorname{cl}\left(B \cap X_{2}\right)$, and (3) follows from (2) since $r\left(P_{i}\right)=r(A)+r(B)-1$ and $r\left(X_{1} \cup X_{2}\right)=r\left(X_{1}\right)+r\left(X_{2}\right)-1$. The case where $P_{i}$ had two joints is similar and is left to the reader.

The proof of the following lemma is straightforward.

Lemma 3.3.28. Let $F$ be a maximal pseudo-flower in $M$.

I) Suppose $P_{i}$ is a 3-petal $F$ in $M$, and let the joints of $P_{i}$ be $j_{1}$ and $j_{2}$. There is a minor $M^{\prime}$ of $M$ such that the following holds.

i) $M^{\prime}=M \backslash A_{1} / A_{2}$ for some $A_{1}, A_{2} \subseteq P_{i}$,

ii) $M^{\prime} \backslash X$ has a maximal swirl-like pseudo-flower $F^{\prime}$, 
iii) $F^{\prime}=\left(P_{1}, \ldots, P_{i-1}, P_{i}^{\prime}, P_{i+1}, \ldots, P_{n}\right)$

iv) every 3-separation of $M^{\prime}$ displayed by $F^{\prime}$ is blocked by some $x \in X$,

v) $P_{i}^{\prime}$ is a triangle $\{a, b, c\}$ with a parallel to $j_{1}$ and $b$ parallel to $j_{2}$ in $\left(M^{\prime}\right)^{+}$.

II) Suppose $P_{i}$ is a joint-based 2-petal $F$ in $M$, and let the joint of $P_{i}$ be $j$. There is a minor $M^{\prime}$ of $M$ such that the following holds.

i) $M^{\prime}=M \backslash A_{1} / A_{2}$ for some $A_{1}, A_{2} \subseteq P_{i}$,

ii) $M^{\prime} \backslash X$ has a maximal swirl-like pseudo-flower $F^{\prime}$,

iii) $F^{\prime}=\left(P_{1}, \ldots, P_{i-1}, P_{i}^{\prime}, P_{i+1}, \ldots, P_{n}\right)$

iv) every 3-separation of $M^{\prime}$ displayed by $F^{\prime}$ is blocked by some $x \in X$,

v) $P_{i}^{\prime}$ is a single element a with a parallel to $j$ in $\left(M^{\prime}\right)^{+}$.

III) Suppose $P_{i}$ is a rim-based 2-petal of $F$ in $M$, and let the joints of $P_{i}$ be $j_{1}$ and $j_{2}$.

i) $M^{\prime}=M \backslash A_{1} / A_{2}$ for some $A_{1}, A_{2} \subseteq P_{i}$,

ii) For some $X \subseteq E\left(M^{\prime}\right)$ the matroid $M^{\prime} \backslash X$ has a maximal swirl-like pseudo-flower $F^{\prime}$,

iii) $F^{\prime}=\left(P_{1}, \ldots, P_{i-1}, P_{i}^{\prime}, P_{i+1}, \ldots, P_{n}\right)$

iv) every 3-separation of $M^{\prime}$ displayed by $F^{\prime}$ is blocked by some $x \in X$,

v) $P_{i}^{\prime}$ is a single element a with a in a triangle with $j_{1}$ and $j_{2}$ in $\left(M^{\prime}\right)^{+}$.

It then easily follows that

Corollary 3.3.29. If $F$ is a maximal swirl-like pseudo-flower of $M$ of order $n$ then $M$ has a wheel minor with $n$ joints.

Lemma 3.3.28 also leads naturally to the following definition:

\section{Definition 3.3.30.}

i) Let $P$ be a 3-petal of $F$ in $M$. The removal of $P$ from $F$ is the matroid obtained by replacing $P$ by a triangle with elements in parallel with the joints of $P$ and contracting the element of this triangle that is not parallel with the joints of $P$. 
ii) If $P$ is a joint-based 2-petal of $F$ in $M$ then the removal of $P$ from $F$ is the matroid obtained by replacing $P$ by an element of $P$ parallel to the joint of $P$. This results in a new swirl-like pseudo-flower whose petals are a subset of the petals of $F$. We denote this flower by $F-P$

iii) If $P$ is a rim-based 2-petal of $F$ in $M$ then the removal of $P$ from $F$ is the matroid obtained by replacing $P$ by an element $e$ of $P$ in a triangle with the joints $j_{1}, j_{2}$ of $P$ and, if $P$ is the only petal with joints $j_{1}$ and $j_{2}$ contracting $e$.

Clearly the removal of $P$ from $F$ gives a minor of $M^{\prime}$ and $F-P$ is a swirl-like pseudo-flower. 


\section{Chapter 4}

\section{Unavoidable Minors of Binary 3- and 4-connected matroids}

In the introduction we stated the unavoidable minors of binary 3- and 4-connected matroids. Now that we know what flowers are it is fairly clear that all these structures are flowers. Obviously it will be useful to us to be able to identify when we have one of these matroids as a minor of another matroid so this section focuses on giving various matrix representations and certificates for these structures.

\subsection{Unavoidable Minors of Binary 3-Connected Matroids}

In [7] it is proved that there is a function $f 1.0 .1$ such that if $M$ is a 3-connected binary matroid with rank at least $f[1.0 .1(n)$ elements, then $M$ has a minor isomorphic to one of $M\left(K_{3, n}\right), M^{*}\left(K_{3, n}\right)$, a rank- $n$ wheel, or a rank- $n$ spike. The next few pages are dedicated to an investigation of these structures since they are also unavoidable minors of binary 4-connected matroids.

\subsection{1 $M\left(K_{3, n}\right)$}

The graph denoted $K_{3, n}$ is the complete bipartite graph with 3 vertices in one part and $n$ in the other. The cycle matroid of $K_{3, n}$ is denoted by $M\left(K_{3, n}\right)$. It is convenient to be able to easily distinguish between the part containing exactly 
three vertices and the other part in writing. Thus we define the top part of $K_{3, n}$ to be the partition containing exactly three vertices and the bottom part to be the other. A 3-connected minor of $K_{3, n}$ is the following graph, which has 3 vertices in the top part and n-2 in the bottom.

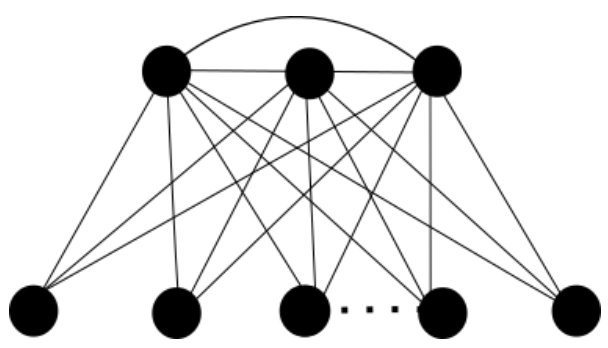

Figure 4.1: $K_{3, n-2}^{+}$

Clearly this graph has a $K_{3, n-2}$ minor. This turns out to be an easier graph to work with leading to the following definition. We call a graph of the form given in Figure 4.1.1 $K_{3, n-2}^{+}$.

Definition 4.1.1. Let $G \cong K_{3, n}^{+}$and $\left\{a_{1}, b_{1}, c_{1}, a_{2}, b_{2}, c_{2}, \ldots, a_{n}, b_{n}, c_{n}\right\}$ be the edges of the $K_{3, n}$ restriction of $G$ and suppose for $i \in\{1, \ldots, n\}$ the edges $a_{i}, b_{i}, c_{i}$ are incident with a single vertex in the bottom part, and $\left\{g_{1}, g_{2}, g_{3}\right\}$ be the edges of $K_{3, n}^{+}$that are not in $K_{3, n}$. A standard representation of $M\left(K_{3, n}^{+}\right)$is a matrix representation of $M\left(K_{3, n}^{+}\right)$of the following form:

\begin{tabular}{|c|c|c|c|c|c|c|c|c|c|}
\hline$a_{1}$ & $c_{1}$ & $a_{2}$ & $c_{2}$ & $a_{3}$ & $c_{3}$ & $\ldots$ & $a_{n}$ & $c_{n}$ & $g_{3}$ \\
\hline$b_{1} / 1$ & 1 & 0 & 0 & 0 & 0 & $\ldots$ & 0 & 0 & 0 \\
\hline 0 & 0 & 1 & 1 & 0 & 0 & $\cdots$ & 0 & 0 & 0 \\
\hline 0 & 0 & 0 & 0 & 1 & 1 & $\ldots$ & 0 & 0 & 0 \\
\hline$\vdots$ & $\vdots$ & $\vdots$ & $\vdots$ & $\vdots$ & $\vdots$ & $\vdots$ & $\vdots$ & $\vdots$ & $\vdots$ \\
\hline 0 & 0 & 0 & 0 & 0 & 0 &. & 1 & 1 & 0 \\
\hline 1 & 0 & 1 & 0 & 1 & 0 & $\vdots$ & 1 & 0 & 1 \\
\hline 0 & 1 & 0 & 1 & 0 & 1 & $\vdots$ & 0 & 1 & 1 \\
\hline
\end{tabular}

A standard basis for $M\left(K_{3, n}^{+}\right)$is a basis for $M\left(K_{3, n}^{+}\right)$that gives a standard representation of $M\left(K_{3, n}^{+}\right)$.

Notice that $M\left(K_{3, n}\right)$ has a flower $F$ where $F=\left(P_{1}, \ldots, P_{n}\right)$ and for $i \in\{1, \ldots, n\}$ the petal $P_{i}=\left\{a_{i}, b_{i}, c_{i}\right\}$, and this flower is a paddle. $M\left(K_{3, n}^{+}\right)$also has a paddle 
and $F=\left(P_{1}, \ldots, P_{n}, P_{n+1}\right)$ where $P_{i}=\left\{a_{i}, b_{i}, c_{i}\right\}$ for $i \in\{1, \ldots, n\}$ and $P_{n+1}=$ $\left\{g_{1}, g_{2}, g_{3}\right\}$. This is the canonical flower of $M\left(K_{3, n}^{+}\right)$. It is worth noting that $\left\{g_{1}, g_{2}, g_{3}\right\} \subseteq \operatorname{cl}\left(P_{i}\right) \cap \operatorname{cl}\left(\left\{\left(P_{1} \cup \cdots \cup P_{n}\right)-P_{i}\right\}\right)$ for any $i \in\{1, \ldots, n\}$.

\subsection{2 $M^{*}\left(K_{3, n}\right)$}

This is the matroid that is the dual of $M\left(K_{3, n}\right)$. Since $K_{3, n}$ is non-planar (for any $n \geq 3)$, the dual of $M\left(K_{3, n}\right)$ is non graphic. Of course $M^{*}\left(K_{3, n}\right)$ is binary and can be represented by a matrix $A$ where

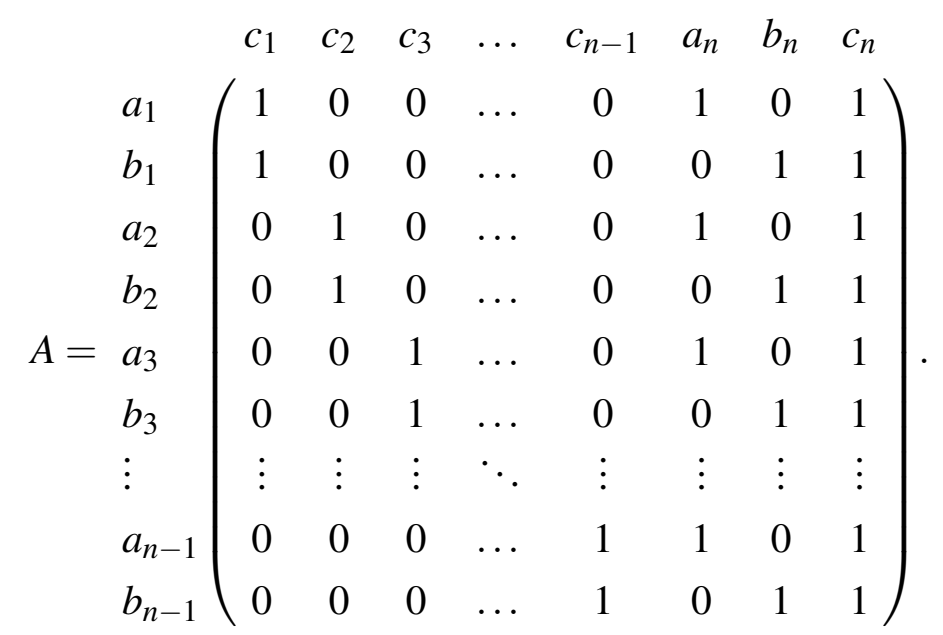

This is the representation we shall be working with most of the time when we are talking about $M^{*}\left(K_{3, n}\right)$ and we shall call this a standard representation of $M^{*}\left(K_{3, n}\right)$. The matroid $M^{*}\left(K_{3, n}\right)$ has a 3-flower $F=\left(P_{1}, P_{2}, P_{3}, \ldots, P_{n}\right)$ where $P_{i}=\left\{a_{i}, b_{i} . c_{i}\right\}$ for $i \in\{1, \ldots, n\}$. Since we know that this flower is a paddle in $M\left(K_{3, n}\right)$, this flower is clearly a copaddle in $M^{*}\left(K_{3, n}\right)$. This is the canonical flower of $M^{*}\left(K_{3, n}\right)$. We call $P_{1}, \ldots, P_{n-1}$ the standard petals of $F$ with respect to $M$ and $P_{n}$ the special petal of $F$ with respect to $M$. Clearly there is really nothing special about the special petal, any petal can be made special by performing a change of basis. However, for a fixed representation, it is useful to be able to distinguish between the petals in this way.

The following lemma gives a useful way of recognising when a matroid is isomorphic to $M^{*}\left(K_{3, n}\right)$.

Lemma 4.1.2. Let $M$ be a matroid and suppose that $E(M)$ can be partitioned into three sets $A, B, C$ such that the following hold. 
1. $|A|=|B|=|C|=n$,

2. $A$ and $B$ are disjoint circuits,

3. $C$ is a set of elements such that every element of $C$ is contained in a triangle with exactly one element of $A$ and exactly one element of $B$ and there is a matching between $A$ and B formed in this way (in other words every element of $A$ and every element of $B$ is contained in exactly one such triangle.)

Then $M \cong M^{*}\left(K_{3, n}\right)$.

Proof. Let $A=\left\{a_{1}, \ldots, a_{n}\right\}, B=\left\{b_{1}, \ldots, b_{n}\right\}$ and $C=\left\{c_{1}, \ldots, c_{n}\right\}$. By relabelling we may assume that $a_{i}, b_{i}, c_{i}$ is a triangle for $i \in\{1, \ldots, n\}$. Clearly $\left(A \backslash a_{n}\right) \cup(B \backslash$ $\left.b_{n}\right)$ is a basis for $M$. It is then clear that $M$ can be represented by :

$$
\begin{aligned}
& \begin{array}{llllllll}
c_{1} & c_{2} & c_{3} & \ldots & c_{n-1} & a_{n} & b_{n} & c_{n}
\end{array}
\end{aligned}
$$

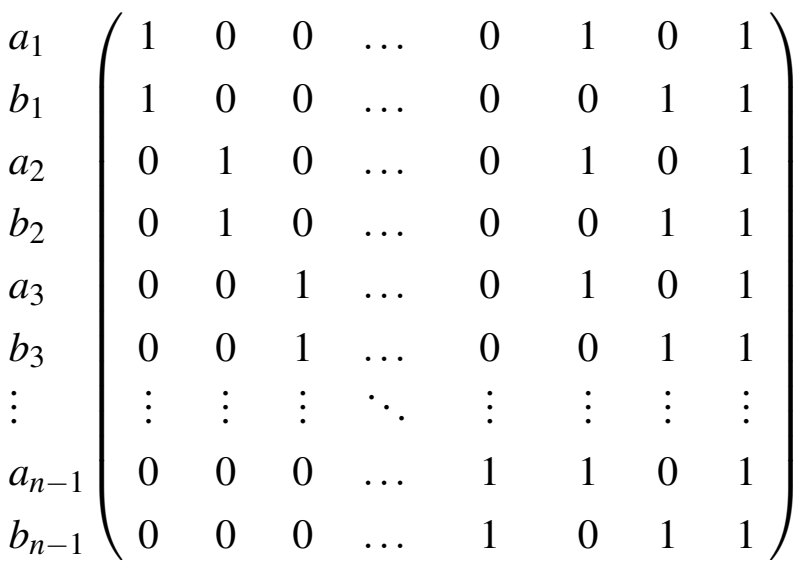

which is a representation for $M^{*}\left(K_{3, n}\right)$.

Clearly the converse of Lemma 4.1.2 is also true.

\subsubsection{Spikes}

For $n \geq 2$, a rank- $n$ spike is a collection of $n$ lines, which we call legs, with exactly two elements on each such that any collection of $n-1$ lines the $n^{\text {th }}$ line is in the span of the other $n-1$. Additionally every two legs form a circuit. These results can be found in [13]. For a rank- $n$ spike with legs $\left\{x_{1}, y_{1}\right\}, \ldots,\left\{x_{n}, y_{n}\right\}$ it is often helpful to draw and visualise the spike as below. 


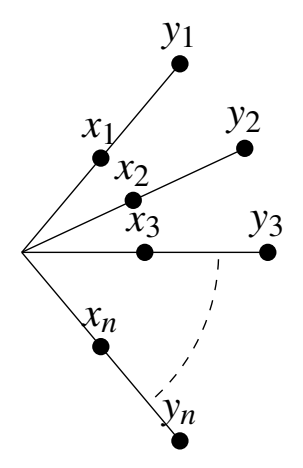

We can choose to add a point in the intersection of the span of all the legs of a spike. If this point is added it is called the tip of the spike. In general for a fixed rank $n$ there are many rank- $n$ spikes. However for any $n \geq 2$ there is a unique binary spike [13](12.2.20).

Definition 4.1.3. A rank- $n$ binary spike is a matroid represented by a $n \times 2 n$ matrix, $A$, of the following form:

$$
\left(\begin{array}{cccccccccc}
1 & 0 & 0 & \ldots & 0 & 0 & 1 & 1 & \ldots & 1 \\
0 & 1 & 0 & \ldots & 0 & 1 & 0 & 1 & \ldots & 1 \\
0 & 0 & 1 & \ldots & 0 & 1 & 1 & 0 & \ldots & 1 \\
\vdots & \vdots & \vdots & \ddots & \vdots & \vdots & \vdots & \vdots & \ddots & \vdots \\
0 & 0 & 0 & \ldots & 1 & 1 & 1 & 1 & \ldots & 0
\end{array}\right)
$$

A rank- $n$ binary spike with tip is a matroid represented by $A \frown[1, \ldots, 1]^{T}$.

The routine proof of the following well-known lemma is left to the reader.

Lemma 4.1.4. If $M$ is a circuit with elements $a, a_{1}, \ldots, a_{n}$ and we extend $M$ by a set $X=\left\{x_{1}, \ldots, x_{n}\right\}$ such that (after possible relabelling) for any $x_{i}$ we have $x_{i} \in \operatorname{cl}\left(\left\{a, a_{i}\right\}\right)$, then $M$ extended by $X$ is a rank-n spike with tip.

\subsubsection{Wheels}

An $n$-spoke wheel is a graph of the following form: 


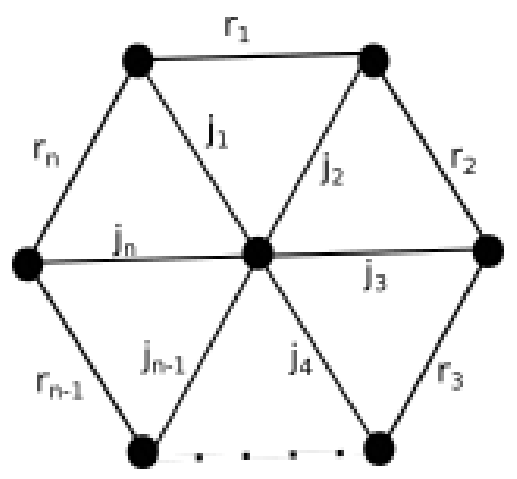

When it is clear from the context that the structure we are discussing is a matroid we shall refer to the cycle matroid of an $n$-spoke wheel as a rank- $n$ wheel. We also sometimes use $M\left(\mathscr{W}_{n}\right)$ to denote the cycle matroid of an $n$-spoke wheel.

A representation of a wheel is given below:

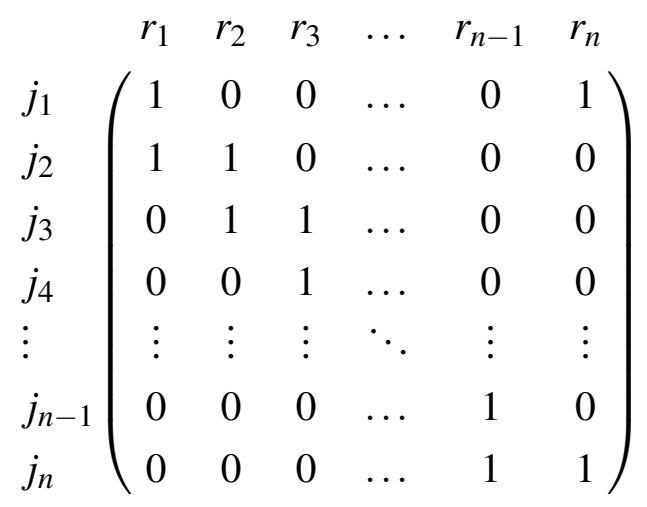

Geometrically, we visualise a wheel in the following way, where $\left\{j_{1}, \ldots, j_{n}\right\}$ is an independent set and $\left\{r_{1}, \ldots, r_{n}\right\}$ is dependent.

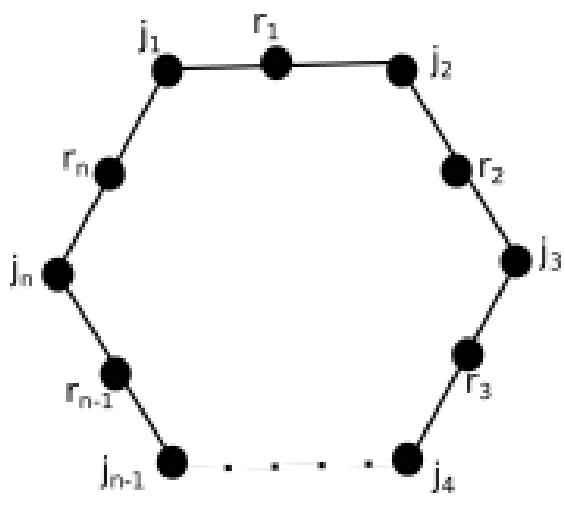

This matroid has flower $\left(P_{1}, \ldots, P_{n}\right)$ with $P_{i}=\left\{j_{i}, r_{i}\right\}$ and this is the canonical flower of a wheel. 


\subsection{Unavoidable Minors of Binary 4-Connected Matroids}

In this section we give details of the matroids that arise as unavoidable minors of 4-connected binary matroids.

\subsubsection{Clams}

We start this section with the most annoying minor we found. These are "clams". A clam is the cycle matroid of the following graph.

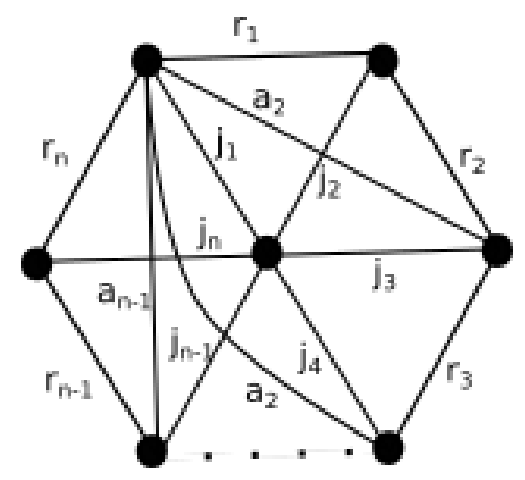

A clam can be represented by the following binary matrix.

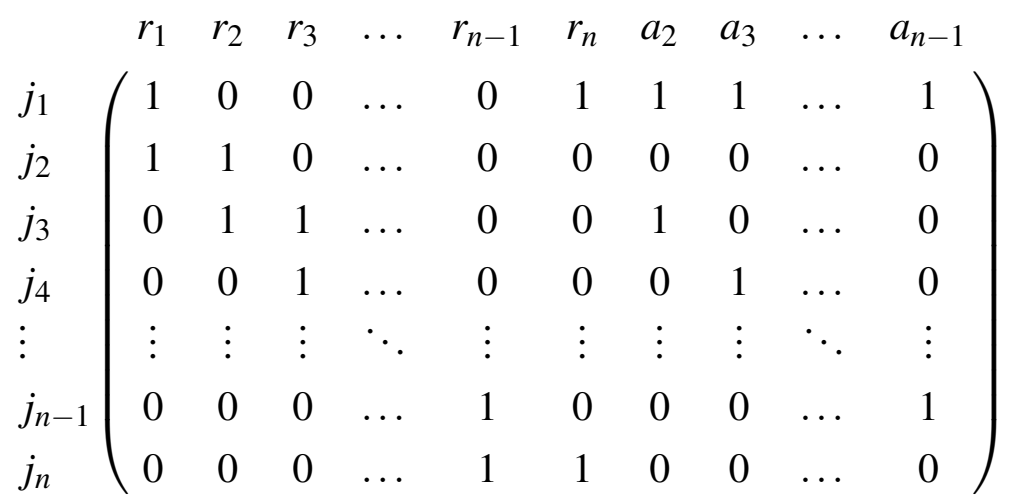

Matroidally we can view a clam in the following way, where $\left\{j_{1}, \ldots, j_{n}\right\}$ is a basis and $\left\{r_{1}, \ldots, r_{n}\right\}$ is a circuit. 


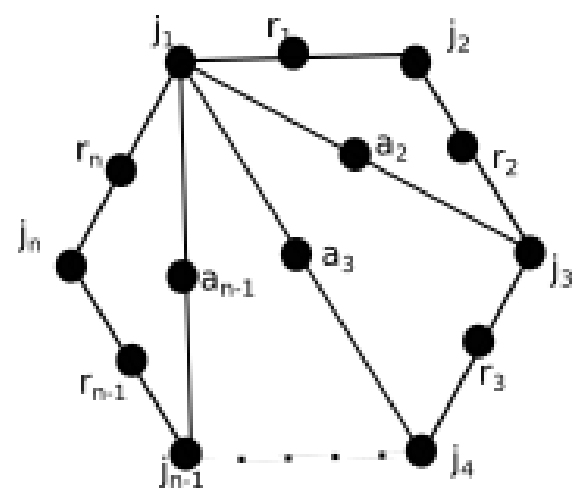

Note that this figure does not include all dependencies, more are forced by the fact the matroid is binary. We shall later see that we get clams as outcomes when we block a wheel in a path-like way. Clams have no induced swirl-like pseudoflowers but, unfortunately, they have many 3-separations. Clams are therefore outcomes that will need to be analysed more thoroughly at a later stage.

\subsubsection{Circular Ladders}

An $n$-rung circular ladder is a graph of the following form:

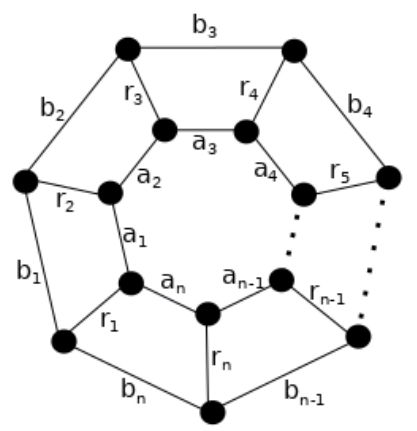

When it is clear from the context that the structure we are discussing is a matroid we shall refer to the cycle matroid of an $n$-rung circular ladder as an $n$-rung circular ladder. Two representations of the matroid of an $n$-rung circular ladder that will 
be useful later are:

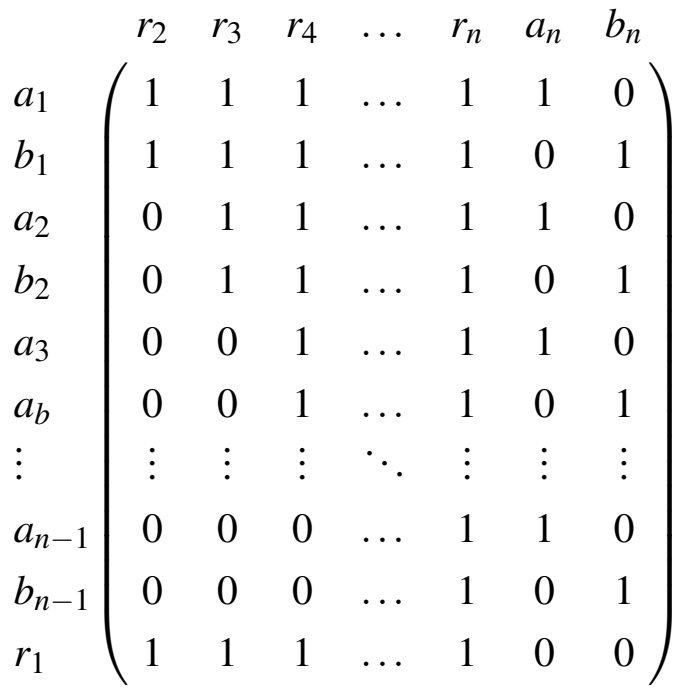

and

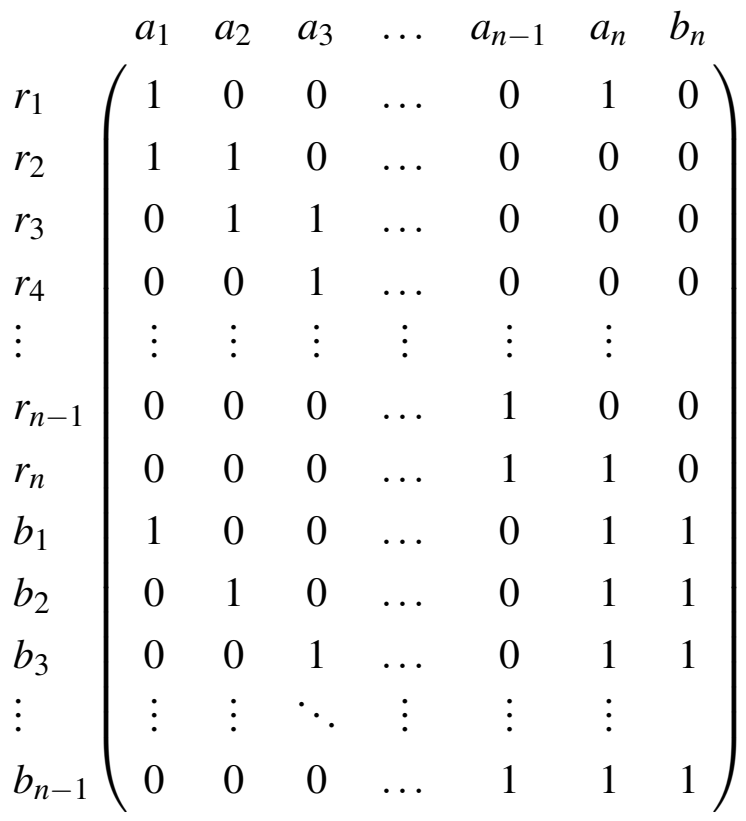

A way of visualising a cycle matroid of a circular ladder is given below. 


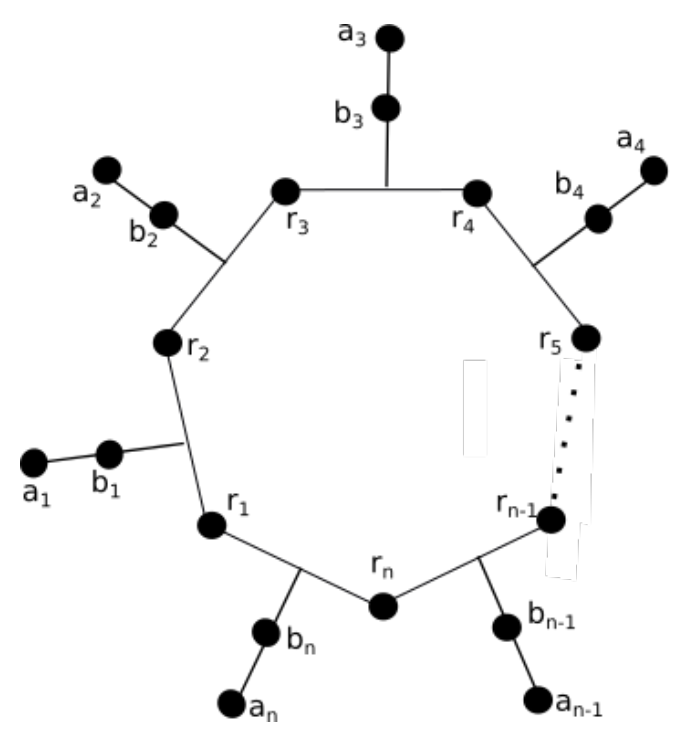

Clearly this is not a geometric representation but it may be helpful in giving some intuition for the matroidal structure.

The proof of the following lemma is clear.

Lemma 4.2.1. An n-rung circular ladder is a 4-flower $\left(P_{1}, \ldots, P_{n}\right)$ where $P_{i}=$ $\left\{r_{i}, a_{i}, b_{i}\right\}$.

An unavoidable minor of an $n$-rung circular ladder is a triangular circular ladder which, in turn, has a circular ladder as a minor. A triangular ladder is a graph of the following form:

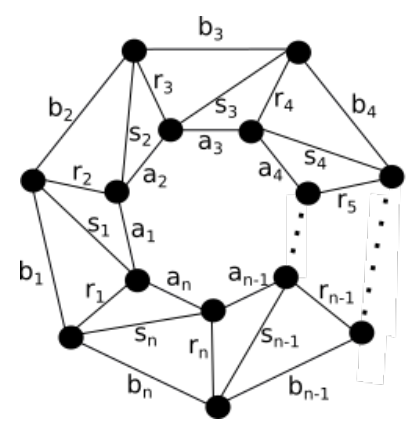


The cycle matroid of a triangular ladder is given below.

$$
\left.\begin{array}{lccccccccc}
a_{1} & b_{1} & a_{2} & \ldots & a_{n-1} & b_{n-1} & a_{n} & b_{n} & s_{n} \\
r_{1} \\
s_{1} \\
r_{2} & 1 & 0 & 0 & \ldots & 0 & 0 & 1 & 0 & 1 \\
s_{2} & 1 & 0 & \ldots & 0 & 0 & 1 & 1 & 1 \\
\vdots & 0 & 1 & 1 & \ldots & 0 & 0 & 1 & 1 & 1 \\
s_{n-1} & 0 & 0 & 1 & \ldots & 0 & 0 & 1 & 1 & 1 \\
r_{n} & \vdots & \vdots & \vdots & \ddots & \vdots & \vdots & \vdots & \vdots & \vdots \\
0 & 0 & 0 & \ldots & 1 & 1 & 1 & 1 & 1 \\
0 & 0 & 0 & \ldots & 0 & 1 & 0 & 1 & 1
\end{array}\right)
$$

This next lemma is helpful in identifying when a matroid is a triangular ladder or has a triangular and hence circular ladder as a minor.

Lemma 4.2.2. Let $M$ be a simple matroid and let $A \subseteq E(M)$ be a circuit. If there is an ordering on the elements of $A$ and the elements of $E(M)-A=B$ such that $a_{i}, b_{i}, a_{i+1}$ is a triangle for all $i \in\{1, \ldots, n-1\}$ and $a_{n}, b_{n}, a_{1}$ is a triangle, then $M$ has a circular ladder with rank $r(M)$ as a minor.

Proof. This follows by letting $A=\left\{r_{1}, s_{1}, \ldots, r_{n}, s_{n}\right\}$, and noticing this gives a triangular circular ladder.

\subsubsection{Möbius Ladders}

An $n$-rung Möbius ladder is a graph of the following form:

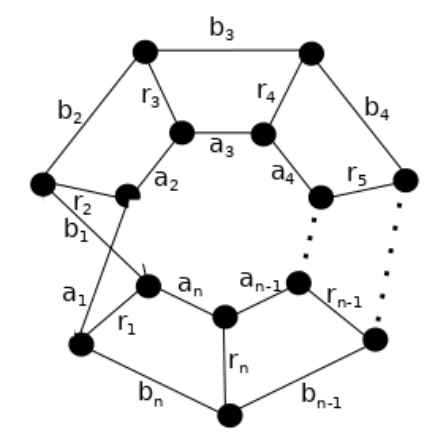

When it is clear from the context that the structure we are discussing is a matroid 
we shall refer to the cycle matroid of an $n$-rung Möbius ladder as an $n$-rung Möbius ladder. Two representations of a Möbius ladder are:

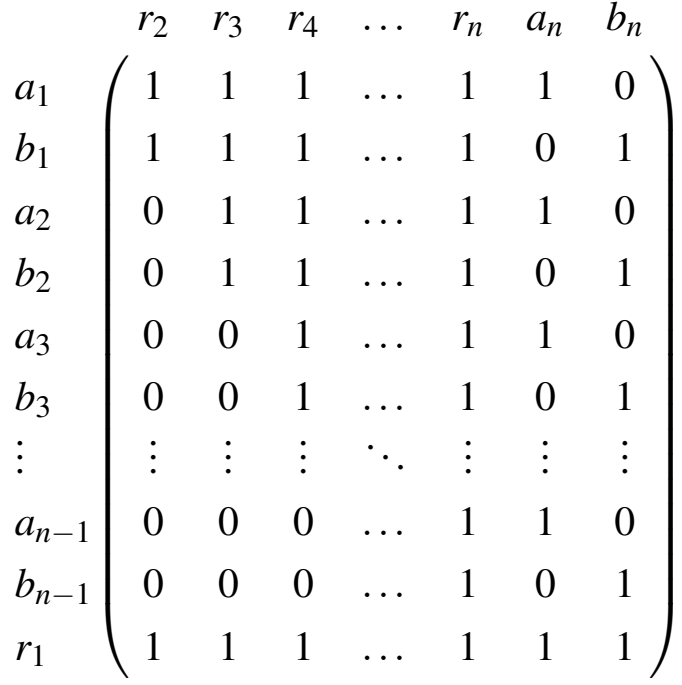

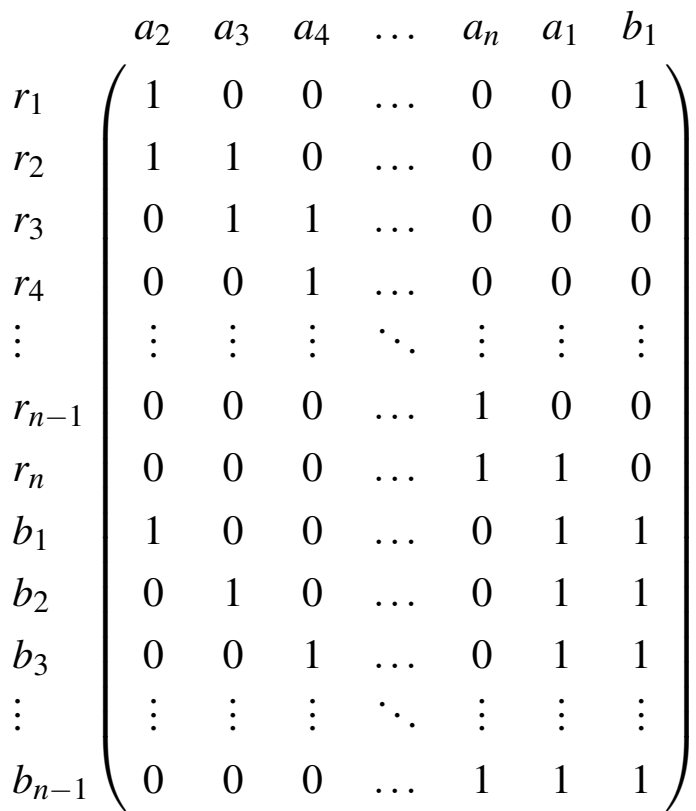

Another drawing of a Möbius ladder graph is: 


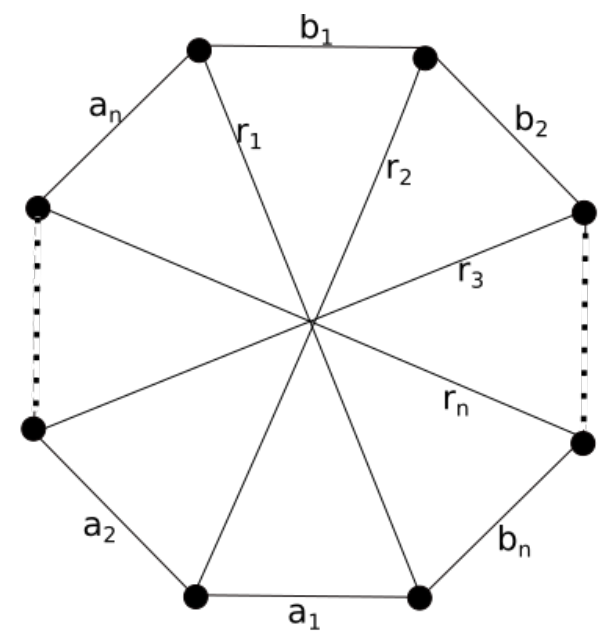

From this it is easy to see that another representation of a cycle matroid of a Möbius ladder is:

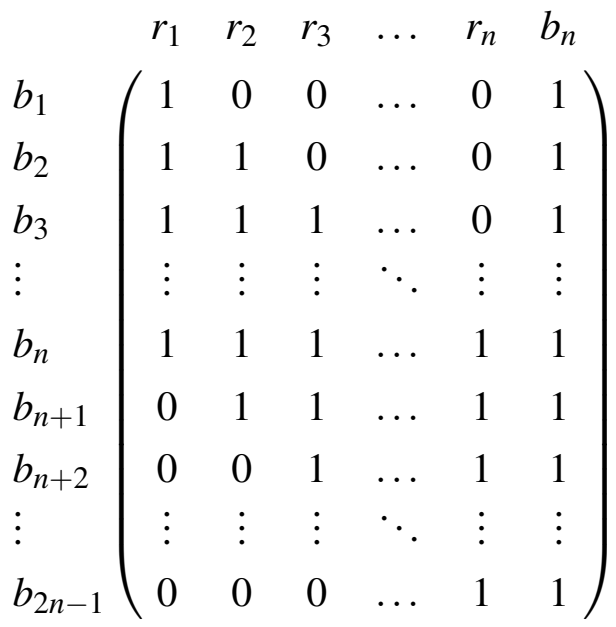

Lemma 4.2.3. An n-rung Möbius ladder is a 4-flower with petals $\left(P_{1}, \ldots, P_{n}\right)$ with $P_{i}=\left\{r_{i}, b_{i}, b_{i+1}\right\}$ where elements are labelled as by the matrix directly above.

\subsubsection{Double Wheels}

A double wheel is a graph of the following form: 


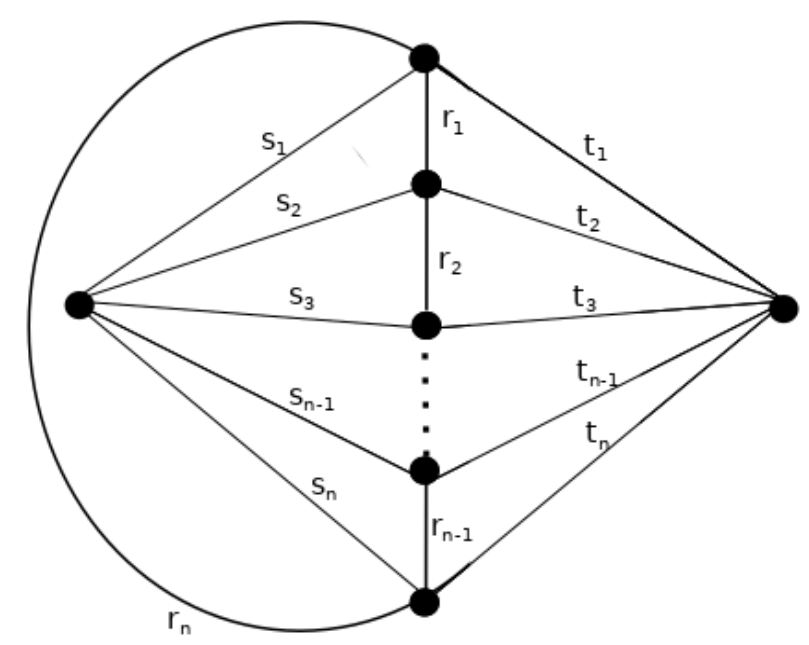

From this we can see that a double wheel is the dual of a circular ladder.

When it is clear from the context that the structure we are discussing is a matroid we shall refer to the cycle matroid of a double wheel as a double wheel. A double wheel can be represented by

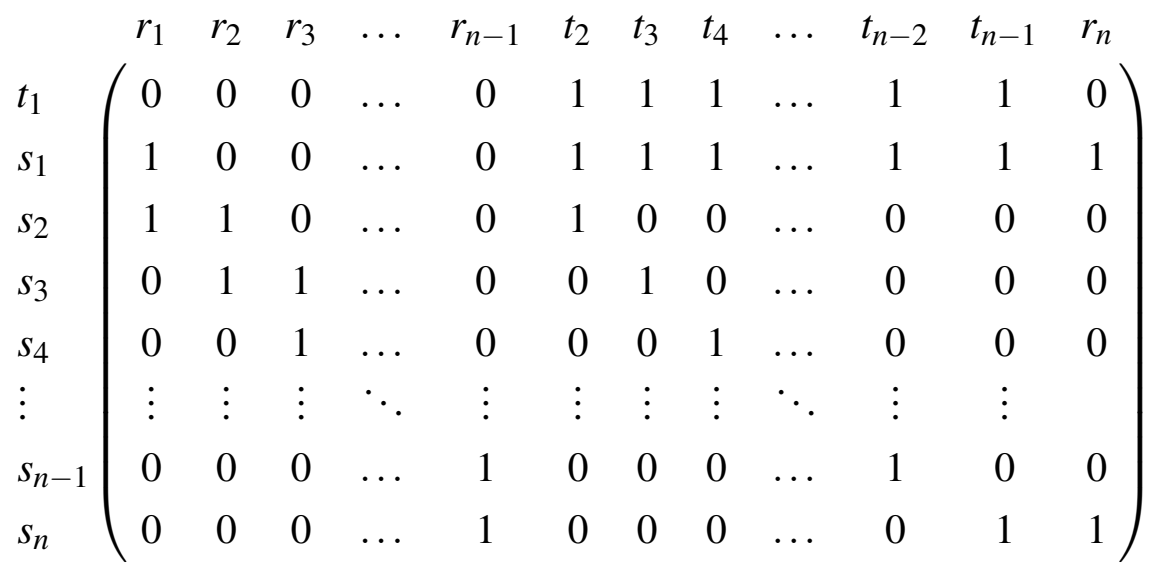

Matroidally we can view a double wheel in the following way: 


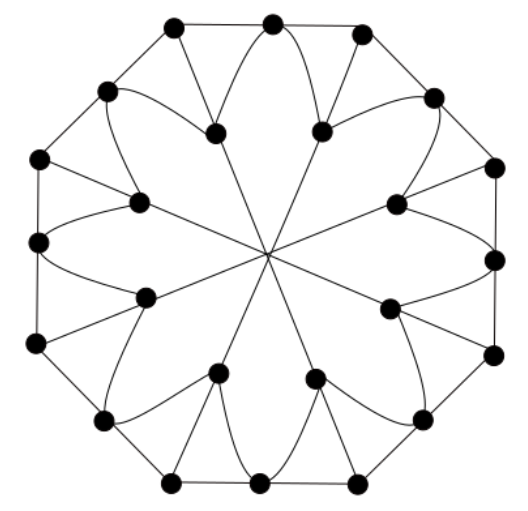

Since this is drawn in rank 3 clearly this is not a traditional matroid drawing. However, it can be helpful in seeing triangles in the matroid.

Lemma 4.2.4. Let $M$ be a double wheel. Then $M$ has a flower $F=P_{1}, \ldots, P_{n}$ with $P_{i}=\left\{r_{i}, s_{i}, t_{i}\right\}$ where the elements in $M$ are labelled as in the matrix above.

\subsubsection{Non-Graphic Double Wheel}

A double wheel is the dual of a circular ladder. Since a double wheel is planar this dual is graphic. However we can also consider duals of Möbius ladders. These structures are very similar to double wheels and we call them non graphic double wheels. A non graphic double wheel can therefore be represented by the following reduced standard representation matrix:

$$
\left(\begin{array}{ccccccccc}
0 & 0 & \ldots & 0 & 1 & 1 & 1 & \ldots & 1 \\
1 & 0 & \ldots & 0 & 0 & 1 & 1 & \ldots & 1 \\
1 & 1 & \ldots & 0 & 0 & 1 & 1 & \ldots & 1 \\
0 & 1 & \ldots & 0 & 0 & 0 & 1 & \ldots & 0 \\
\vdots & \vdots & \ddots & \vdots & \vdots & \vdots & \vdots & \ddots & \vdots \\
0 & 0 & \ldots & 1 & 0 & 0 & 0 & \ldots & 0 \\
0 & 0 & \ldots & 1 & 1 & 0 & 0 & \ldots & 1
\end{array}\right)
$$

or, equlvalently 


$$
\left(\begin{array}{cccccccccc}
0 & 0 & 0 & \ldots & 1 & 1 & 1 & \ldots & 1 & 1 \\
1 & 0 & 0 & \ldots & 1 & 1 & 1 & \ldots & 1 & 0 \\
1 & 1 & 0 & \ldots & 0 & 1 & 0 & \ldots & 0 & 0 \\
0 & 1 & 1 & \ldots & 0 & 0 & 1 & \ldots & 0 & 0 \\
\vdots & \vdots & \vdots & \ddots & \vdots & \vdots & \vdots & \ddots & \vdots & \vdots \\
0 & 0 & 0 & \ldots & 1 & 0 & 0 & \ldots & 1 & 1
\end{array}\right)
$$

The following picture gives a way of visualising a non graphic double wheel.

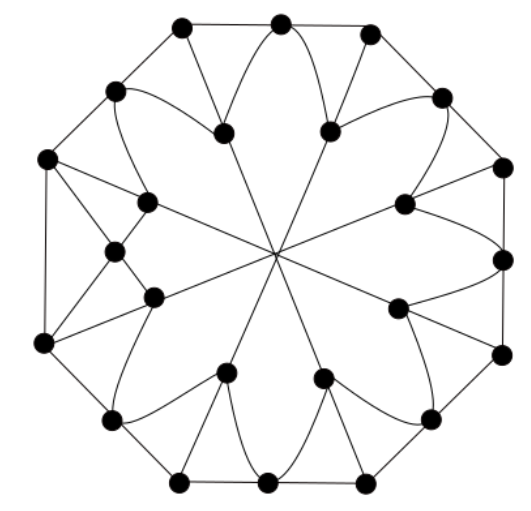

Again, this is not a geometric representation of the matroid, but does show many of the triangles.

\subsection{6 $M\left(K_{4, n}\right)$}

$K_{4, n}$ is the complete bipartite graph with four vertices in one part of the partition and $n$ vertices in the other. As with $K_{3, m}$ we can find a minor of $K_{4, n}$ of the following form:

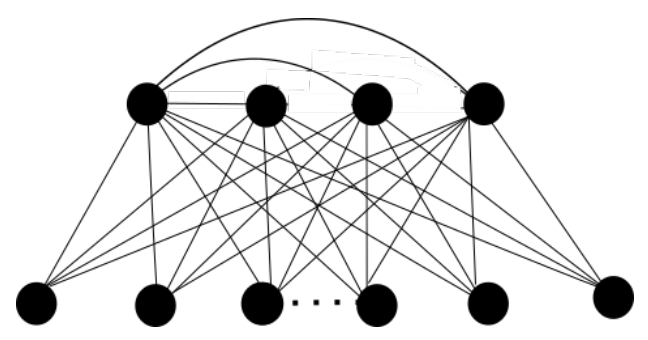

When there are $n$ vertices in the bottom part this has $K_{4, n}$ as a minor. The matroid of this graph can be represented by the reduced standard representation matrix 
below.

$$
\left(\begin{array}{cccccccccc}
1 & 1 & 1 & 0 & 0 & 0 & \ldots & 0 & 0 & 0 \\
0 & 0 & 0 & 1 & 1 & 1 & \ldots & 0 & 0 & 0 \\
\vdots & \vdots & \vdots & \vdots & \vdots & \vdots & \ddots & \vdots & \vdots & \vdots \\
0 & 0 & 0 & 0 & 0 & 0 & \ldots & 1 & 1 & 1 \\
1 & 0 & 0 & 1 & 0 & 0 & \ldots & 1 & 0 & 0 \\
0 & 1 & 0 & 0 & 1 & 0 & \ldots & 0 & 1 & 0 \\
0 & 0 & 1 & 0 & 0 & 1 & \ldots & 0 & 0 & 1
\end{array}\right)
$$

\subsection{7 $M^{*}\left(K_{4, n}\right)$}

Of course the dual of $M\left(K_{4, n}\right)$ is also an unavoidable minor of binary 4-connected matroids. The matroid $M^{*}\left(K_{4, n}\right)$ can be represented by the following reduced standard representation matrix:

$$
\left(\begin{array}{cccccccc}
1 & 0 & 0 & \ldots & 0 & 1 & 0 & 0 \\
1 & 0 & 0 & \ldots & 0 & 0 & 1 & 0 \\
1 & 0 & 0 & \ldots & 0 & 0 & 0 & 1 \\
0 & 1 & 0 & \ldots & 0 & 1 & 0 & 0 \\
0 & 1 & 0 & \ldots & 0 & 0 & 1 & 0 \\
0 & 1 & 0 & \ldots & 0 & 0 & 0 & 1 \\
0 & 0 & 1 & \ldots & 0 & 1 & 0 & 0 \\
0 & 0 & 1 & \ldots & 0 & 0 & 1 & 0 \\
0 & 0 & 1 & \ldots & 0 & 0 & 0 & 1 \\
\vdots & \vdots & \vdots & \ddots & \vdots & \vdots & \vdots & \vdots \\
0 & 0 & 0 & \ldots & 0 & 1 & 0 & 0 \\
0 & 0 & 0 & \ldots & 0 & 0 & 1 & 0 \\
0 & 0 & 0 & \ldots & 0 & 0 & 0 & 1
\end{array}\right)
$$




\subsection{8 $N\left(K_{3, n}\right)$}

Definition 4.2.5. Let $M$ be a matroid with reduced standard representation matrix given below.

$$
\left(\begin{array}{cccccccccc}
1 & 1 & 0 & 0 & 0 & 0 & \ldots & 0 & 0 & 1 \\
0 & 0 & 1 & 1 & 0 & 0 & \ldots & 0 & 0 & 1 \\
0 & 0 & 0 & 0 & 1 & 1 & \ldots & 0 & 0 & 1 \\
\vdots & \vdots & \vdots & \vdots & \vdots & \vdots & \ddots & \vdots & \vdots & \vdots \\
0 & 0 & 0 & 0 & 0 & 0 & \ldots & 1 & 1 & 1 \\
1 & 0 & 1 & 0 & 1 & 0 & \ldots & 1 & 0 & 1 \\
0 & 1 & 0 & 1 & 0 & 1 & \ldots & 0 & 1 & 1
\end{array}\right) .
$$

The matroid $N\left(K_{3, n}\right)$ is defined to be $M / x$.

This operation is similar to the operation used to obtain a spike from $M\left(K_{2, n}\right)$. We may obtain a spike from $M\left(K_{2, n}\right)$ as follows. First consider a flower, $F=$ $\left(P_{1}, \ldots, P_{n}\right)$, of $M\left(K_{2, n}\right)$ where $P_{i}$ consists of the pair of edges joining a vertex $v_{i}$ in the bottom part to one of the vertices in the top part, We can add a point $x$ to $M \cong M\left(K_{2, n}^{+}\right)$that is in the span of the union of all the petals, but is not in the span of any strict subset of the petals. The element $x$ blocks all internal 3-separations of $M$ and when we contract $x$ we obtain a spike. There are various places we can put $x$ that satisfy the conditions, and these give rise to the different spikes. However there is only one place for $x$ in binary space and this gives rise to the (unique) binary spike. The construction described for spikes can be extended to any $M\left(K_{m, n}\right)$, and the construction of $N\left(K_{3, n}\right)$ given in the definition is such a construction for $M\left(K_{3, n}\right)$.

\subsubsection{Speels}

Definition 4.2.6. A speel is a matroid represented by the following reduced standard representation matrix. 


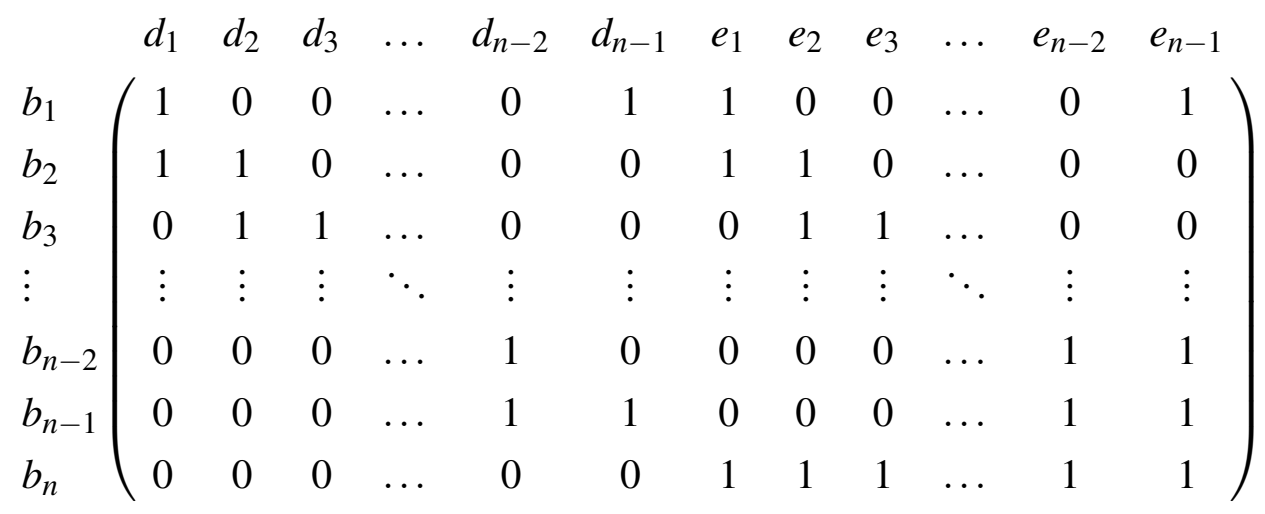

A rank- $n$ speel is pictured below:

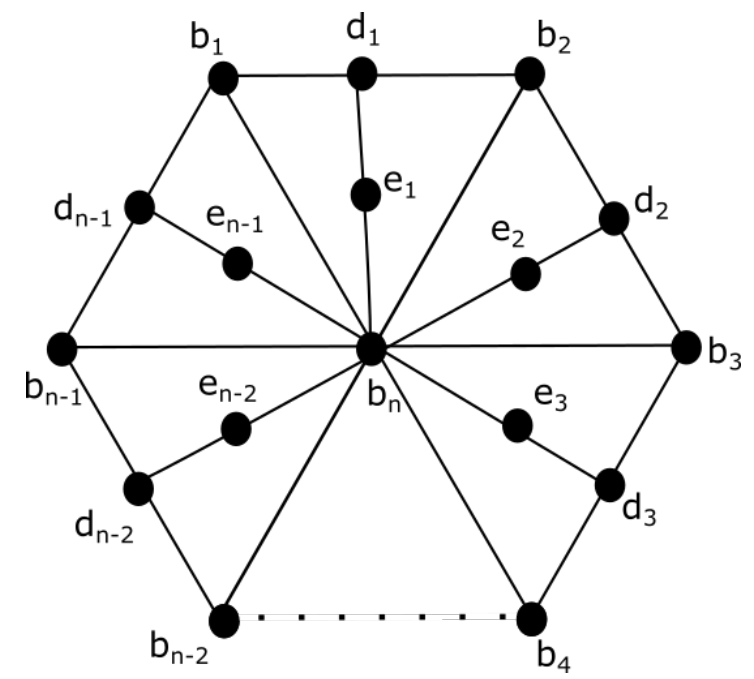

It is immediately clear from the matrix that if $M$ is a speel then $M \mid\left\{b_{1}, \ldots, b_{n-1}, d_{1}, \ldots, d_{n-1}\right\}$ is a wheel. It is slightly less clear that $M \mid\left\{d_{1}, e_{1}, d_{2}, e_{2}, \ldots, d_{n-1}, e_{n-1}\right\}$ is a spike. Since we are excluding a spike as a minor of the matroids we are considering this outcome does not come up in the thesis. However it will prove to be a matroid that is an element of the set of unavoidable minors of binary 4-connected matroids, and we expect to see this when we consider blocking spikes. 


\section{Chapter 5}

\section{Blocking a Paddle}

Recall that a set $X$ blocks a flower $F$ in a matroid $M$ if every 3-separation of $M$ displayed by $F$ is blocked by some $x \in X$.

The main result of this chapter is the following.

Theorem 5.0.1. There is a function 5 5.0.1 such that the following holds. Suppose $M$ is a binary matroid such that for some coindependent set $X$, the matroid $M \backslash X$ has a paddle $F=\left(P_{1}, \ldots, P_{m}\right)$ with at least $n$ proper petals. Further suppose that $X$ is such that every 3-separation of $M \backslash X$ displayed by $F$ is blocked by some $x \in X$. If $n \geq \sqrt{5.0 .1}(t)$, then $M$ has a minor isomorphic to one of the following:
i) $N\left(K_{3, t}\right)$
ii) $M\left(K_{4, t}\right)$,
iii) a rank-t double wheel.

In this chapter we work under the hypotheses of Theorem 5.0.1. That is we work under the following hypotheses.

- $M$ is a binary matroid,

- $X \subseteq E(M)$ is a coindependent set such $M \backslash X$ has a paddle $F$ with at least $n$ proper petals,

- every 3-separation of $M \backslash X$ displayed by $F$ is blocked by some $x \in X$,

- $X=\left\{x_{1}, \ldots, x_{l}\right\}$, 
We also lose no generality by assuming that $X$ is minimal with respect to $\left(^{*}\right)$ so we add the following hypothesis.

- $X$ is minimal with respect to (*).

We call $X$ the set of blocking elements for $F$.

In the first section we set up some matrices that represent unavoidable minors of $M$. The remaining sections simplify these matrices to get a proof of Theorem 5.0.1 The second section in this chapter will consider the case where $M \backslash X \cong M\left(K_{3, n}\right)$. The third section considers the case when $M \backslash X$ is an arbitrary paddle. We will often be able to be reduced to the case where $M \backslash X \cong M\left(K_{3, n}\right)$. The final section gives a proof of Theorem 5.0.1.

If $F$ is a paddle and does not have a guts petal we may delete and contract elements of some petal of $F$ to obtain a guts petal. We may therefore, without loss of generality, assume that $F$ has a guts petal. We now add the following hypotheses.

- $F$ has guts petal $G$ and $F=\left(P_{1}, \ldots, P_{n}, G\right)$, where $P_{1}, \ldots, P_{n}$ all have rank at least 3 in $M$.

- $B$ is a basis for $M$ consisting of a spanning subset of $G$ and at least one element from every $P_{i}$ for $i \in\{1, \ldots, n\}$

\subsection{Setting Up Some Matrices}

Lemma 5.1.1. If $P$ is a petal of $F$ containing an element of $F_{B}(x)$ that is not in $\langle G\rangle$, then $P$ is blocked by $x$; and, if $P$ is blocked by $x$, then $P$ contains an element of the fundamental circuit of $x$ with respect to $B$ that is not in $\langle G\rangle$. Moreover, if $\mathscr{P}$ is the set of petals blocked by $x$, then $\mathscr{P}$ is the unique minimal set of petals containing $x$ in its closure.

Proof. Let $F_{B}(x)=C_{x}$ and $N=M \backslash X$. Suppose $e \in C_{x}-\langle G\rangle$. For some $i \in$ $\{1, \ldots, n\}$ we have $e \in P_{i}$ and, since $C_{x}$ cannot be contained in a single petal of $F$, $x \notin \operatorname{cl}\left(P_{i}\right)$. Similarly, since $e \in\left(C_{x}-\operatorname{cl}(G)\right) \cap P_{i}$, we observe that $C_{x} \nsubseteq \mp F-P_{i}$, and therefore $x \notin\left\langle\left(E(N)-P_{i}\right)\right\rangle$. It then follows immediately that $x$ blocks $P_{i}$. Now suppose $x$ blocks $P_{i}$. Then $x \notin\left\langle\left(E(N)-P_{i}\right)\right\rangle$ and $x \notin\left\langle\left(P_{i}\right)\right\rangle$. Therefore there is 
some $e \in P_{i}$ that is contained in $C_{x}$. This concludes to proof of the first part of the result.

We now prove that if $\mathscr{P}$ is the set of petals blocked by $x$ then $\cup \mathscr{P}$ is the unique minimal set of petals containing $x$ in its closure.

No subset of $\cup \mathscr{P}$ contains $x$ in the closure and by the first half of the lemma $F(x) \subseteq(\cup \mathscr{P}) \cup G$. By definition of $G$, we see $G \in \operatorname{cl}\left(P_{i}\right)$ for $i \in\{1, \ldots, l\}$ so $x$ is in the minimal closure of $\cup \mathscr{P}$. Now suppose there is some set $\mathscr{Q}$ of petals such that $\mathscr{Q} \nsupseteq \mathscr{P}$ and $x \in \operatorname{cl}(\mathscr{Q})$. It would then follow that $F(x) \subseteq \cup \mathscr{Q}$, a contradiction.

Consider the matroid $M$. From $M$ we construct a matrix $\Gamma$ with rows labelled by $P_{i}^{\prime}$ and columns labelled by $x_{j}^{\prime}$ for $i \in\{1, \ldots, n\}$ and $j \in\{1, \ldots, l\}$ as follows.

$$
\Gamma_{P_{i}^{\prime}, x_{j}}=\left\{\begin{array}{l}
1 \text { if } C_{x_{j}} \text { contains an element from } P_{i} \\
0 \text { otherwise }
\end{array}\right.
$$

Thus we have a matrix over $G F(2)$ in which every column contains at least two ones (since we cannot block a single petal) and no two columns are identical (as this would mean two elements blocked the same set of petals). Since we may permute columns, delete rows and delete columns, Lemma 2.4.9 tells us that if $\Gamma$ is sufficiently large there is a large submatrix, $\Gamma^{\prime}$, of $\Gamma$ obtained by deleting rows and columns and permuting rows and columns so that $\Gamma^{\prime}$ is of the following forms:

$$
\left(\begin{array}{c}
1 \\
1 \\
1 \\
1 \\
1 \\
1 \\
\vdots \\
1
\end{array}\right)
$$




$$
\begin{gathered}
\left(\begin{array}{ccccccc}
1 & 1 & 1 & 1 & 0 & \ldots & 1 \\
1 & 0 & 0 & 0 & 0 & \ldots & 0 \\
0 & 1 & 0 & 0 & 0 & \ldots & 0 \\
0 & 0 & 1 & 0 & 0 & \ldots & 0 \\
0 & 0 & 0 & 1 & 0 & \ldots & 0 \\
0 & 0 & 0 & 0 & 1 & \ldots & 0 \\
\vdots & \vdots & \vdots & \vdots & \vdots & \ldots & \vdots \\
0 & 0 & 0 & 0 & 0 & \ldots & 1
\end{array}\right) \\
\left(\begin{array}{cccccc}
1 & 0 & 0 & 0 & \ldots & 0 \\
1 & 1 & 0 & 0 & \ldots & 0 \\
0 & 1 & 1 & 0 & \ldots & 0 \\
0 & 0 & 1 & 1 & \ldots & 0 \\
0 & 0 & 0 & 1 & \ldots & 0 \\
\vdots & \vdots & \vdots & \vdots & \ldots & \vdots \\
0 & 0 & 0 & 0 & \ldots & 1 \\
0 & 0 & 0 & 0 & \ldots & 1
\end{array}\right)
\end{gathered}
$$

or $\Gamma^{\prime}$ has a block decomposition into $m$ blocks where the only non-zero entries occur in the diagonal blocks.

Since any 3-separation of $M$ displayed by $F$ is blocked by some $x \in X$, the case where the matrix has the block decomposition described above does not arise.

Consider a representation of $M$ with respect to basis $B$, and consider the set $X$ of blocking elements. The matroid $M \backslash X$ is a paddle $\left(P_{1}, \ldots, P_{n}, G\right)$ for $G \subseteq \operatorname{cl}\left(P_{i}\right)$ for $i \in\{1, \ldots, n\}$ and so can be represented by a matrix $\Delta$ of the following form:

$$
\left(\begin{array}{ccccc}
P_{1}^{\prime} & 0 & 0 & \ldots & 0 \\
0 & P_{2}^{\prime} & 0 & \ldots & 0 \\
0 & 0 & P_{3}^{\prime} & \ldots & 0 \\
\vdots & \vdots & \vdots & \ddots & \vdots \\
0 & 0 & 0 & \ldots & P_{n}^{\prime} \\
G_{1} & G_{2} & G_{2} & \ldots & G_{n}
\end{array}\right)
$$

where, for $i \in\{1, \ldots, n\}, P_{i}^{\prime}$ and $G_{i}$ are matrices such that the following hold.

i) $G_{i}$ has two rows and the rows of $G_{i}$ are labelled by the elements in $G$, 
ii) $G_{1}, \ldots, G_{n}$ represent matrices that each contain at least one non-zero entry in every row,

iii) the rows of $P_{i}^{\prime}$ are labelled by $B \cap\left(M \mid P_{i}\right)$, and

iv) the columns containing columns of $P_{i}^{\prime}$ label the elements of $P_{i}-B$.

In $\Gamma$ we may consider a " 1 " in row $P_{i}^{\prime}$ to represents a $\left(\left|r\left(P_{i}\right)\right|-2\right) \times 1$ matrix, $\Gamma_{i}$, where the rows of $\Gamma_{i}$ are labelled by the basis elements of $P_{i}$ and there is at least one "1" in some row of $\Gamma_{i}$. Call the matrix constructed from $\Gamma$ in this way $\widetilde{\Gamma}$. Since we are working with binary matrices, $\Delta \frown \widetilde{\Gamma}$ is a reduced standard representation matrix for $M$. In this way we get the following lemma.

Lemma 5.1.2. If $n \geq \sqrt{2.4 .9}(t)$, then there is a minor $M^{\prime}$ of $M$ such that $M^{\prime} \backslash(X \cap$ $\left.E\left(M^{\prime}\right)\right)$ has a paddle $F^{\prime} \subseteq F$ with at least $t+1$ petals which, after possible relabelling, can be represented by one of the following matrices:

$$
\left(\begin{array}{cccccc}
P_{1}^{\prime} & 0 & 0 & \ldots & 0 & \Gamma_{1} \\
0 & P_{2}^{\prime} & 0 & \ldots & 0 & \Gamma_{2} \\
0 & 0 & P_{3}^{\prime} & \ldots & 0 & \Gamma_{3} \\
\vdots & \vdots & \vdots & \ddots & \vdots & \vdots \\
0 & 0 & 0 & \ldots & P_{t}^{\prime} & \Gamma_{t} \\
G_{1} & G_{2} & G_{2} & \ldots & G_{t} & ?
\end{array}\right)
$$

(a)

$$
\left(\begin{array}{ccccccccc}
P_{1}^{\prime} & 0 & 0 & \ldots & 0 & \Gamma_{1} & \Gamma_{2} & \ldots & \Gamma_{t-1} \\
0 & P_{2}^{\prime} & 0 & \ldots & 0 & \Gamma_{1}^{\prime} & 0 & \ldots & 0 \\
0 & 0 & P_{3}^{\prime} & \ldots & 0 & 0 & \Gamma_{2}^{\prime} & \ldots & 0 \\
\vdots & \vdots & \vdots & \ddots & \vdots & \vdots & \vdots & \ddots & \vdots \\
0 & 0 & 0 & \ldots & P_{t}^{\prime} & 0 & 0 & \ldots & \Gamma_{3}^{\prime} \\
G_{1} & G_{2} & G_{3} & \ldots & G_{t} & ? & ? & \ldots & ?
\end{array}\right)
$$




$$
\left(\begin{array}{cccccccccc}
P_{1}^{\prime} & 0 & 0 & \ldots & 0 & 0 & \Gamma_{1} & 0 & \ldots & 0 \\
0 & P_{2}^{\prime} & 0 & \ldots & 0 & 0 & \Gamma_{1}^{\prime} & \Gamma_{2} & \ldots & 0 \\
0 & 0 & P_{3}^{\prime} & \ldots & 0 & 0 & 0 & \Gamma_{2}^{\prime} & \ldots & 0 \\
\vdots & \vdots & \vdots & \ddots & \vdots & \vdots & \vdots & \vdots & \ddots & \vdots \\
0 & 0 & 0 & \ldots & P_{t-1}^{\prime} & 0 & 0 & 0 & \ldots & \Gamma_{t-1} \\
0 & 0 & 0 & \ldots & 0 & P_{t}^{\prime} & 0 & 0 & \ldots & \Gamma_{t-1}^{\prime} \\
G_{1} & G_{2} & G_{3} & \ldots & G_{t-1} & G_{t} & ? & ? & \ldots & ?
\end{array}\right)
$$

(c)

Where, for $i \in\{1, \ldots, n\}, P_{i}^{\prime}$ and $G_{i}$ are matrices where

1. $G_{i}$ has two rows and the rows of $G_{i}$ are labelled by the elements in the guts petal of $P$.

2. $G_{1}, \ldots, G_{n}$ represent matrices that each contain at least one non-zero entry in every row.

3. The rows of $P_{i}^{\prime}$ are labelled by the basis elements of $\left(M \mid P_{i}\right)$ and

4. The columns containing columns of $P_{i}^{\prime}$ label the elements of $P_{i}-B$.

5. $\Gamma_{i}, \Gamma_{i}^{\prime}$ represent $\left(\left|r\left(P_{i}\right)\right|-2\right) \times 1$ matrices with rows labelled by the basis elements of $P_{i}$ and for every $i \in\{1, \ldots, n\}$ there is a 1 in some row of $\Gamma_{i}$.

We can now split the analysis for blocking a paddle into three cases, one case for each of the matrices above.

For the remainder of this chapter we work under the following hypothesis.

- The matroid $M$ can be represented by one of $(a),(b),(c)$ from Lemma 5.1.2.

- $\Gamma$ is the matrix whose construction is described in 5.1.1.

- $\Delta$ is the matrix representing $M \backslash X$ with respect to basis $B$, and

- $\Lambda$ is the matrix representing $M$ with respect to basis $B$ and $\Lambda=\Delta \frown \Gamma$. 


\subsection{Blocking $M\left(K_{3, n}^{+}\right)$}

In this section we focus on the special case where $M \backslash X \cong M\left(K_{3, n}^{+}\right)$. This will be useful in the next section since in the general case there is often a minor of $M$ isomorphic to $M^{\prime}$ where $M^{\prime} \backslash X \cong M\left(K_{3, n}^{+}\right)$and every 3-separation displayed by the canonical flower of $M^{\prime} \backslash X$ is blocked by an element of $X$.

For the remainder of this section we are working under the following hypotheses:

- $M \backslash X \cong M\left(K_{3, n}^{+}\right)$,

- $F$ is the canonical flower of $M\left(K_{3, n}^{+}\right)$.

Lemma 5.2.1. If $n \geq \sqrt{2.4 .9}(t)$, then there is a minor of $M$ of rank at least $t+2$ that can be represented by one of the following matrices:

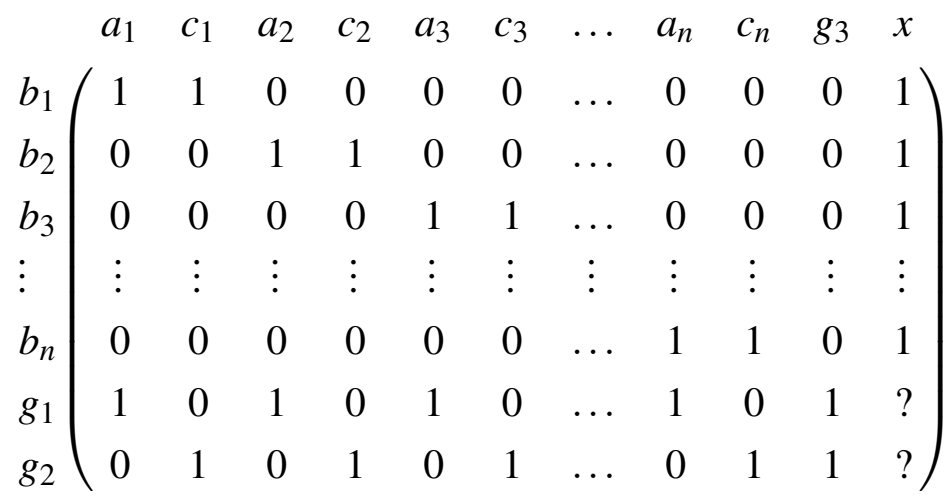

$\left(a^{\prime}\right)$

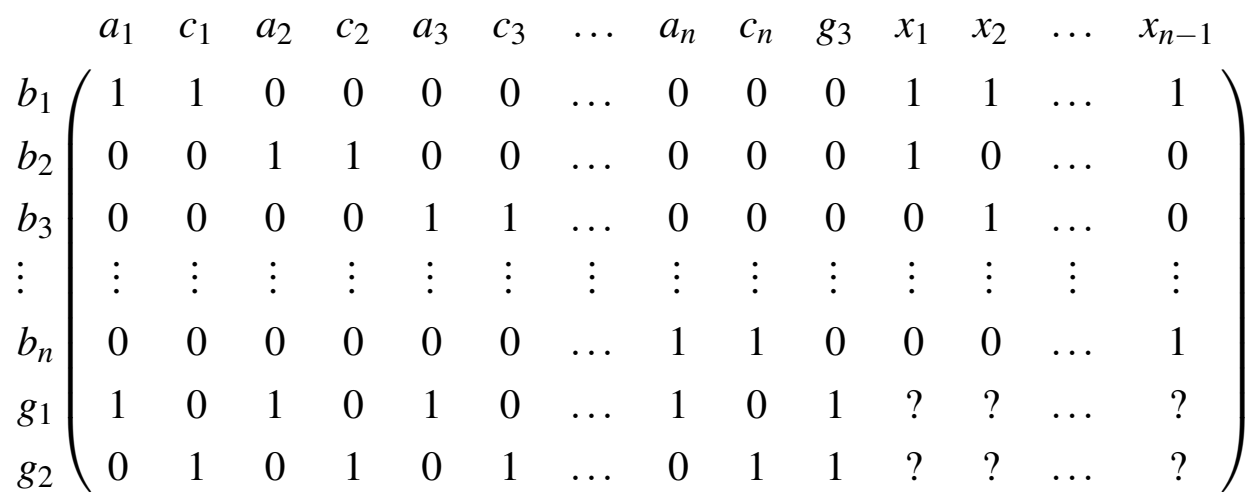




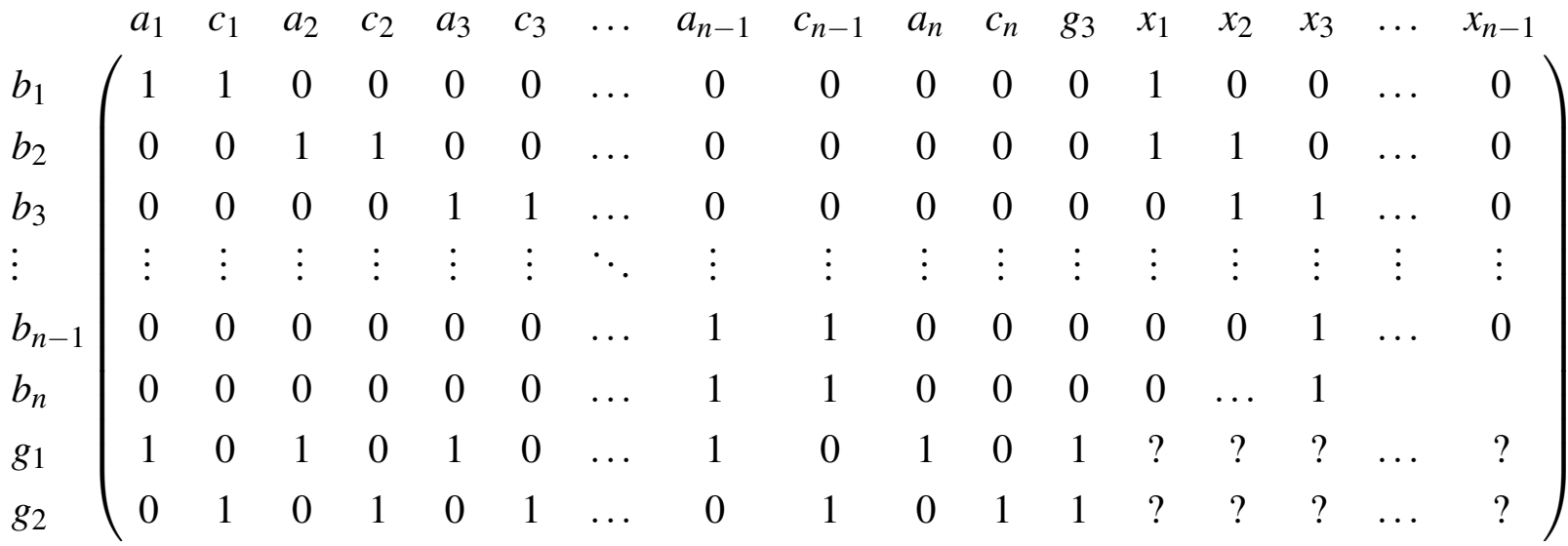

$$
\begin{aligned}
& \text { (c') }
\end{aligned}
$$

Proof. This follows from Lemma 5.1.2.

This means, that for this section, we assume that $\Lambda$ is of form $\left(a^{\prime}\right),\left(b^{\prime}\right)$ or $\left(c^{\prime}\right)$. We now split the analysis of the the case of blocking $M\left(K_{3, n}^{+}\right)$into three cases, one case for each of the matrices above.

\section{Case $\left(a^{\prime}\right)$}

In this section we are considering the case where $\Lambda$ is of from $\left(a^{\prime}\right)$. That is we are considering the case where $|X|=1$, so there is a single blocking element, $x$, that blocks all 3-separations of $M \backslash x$ displayed by $F$. In this case we assume that $M$ is represented by

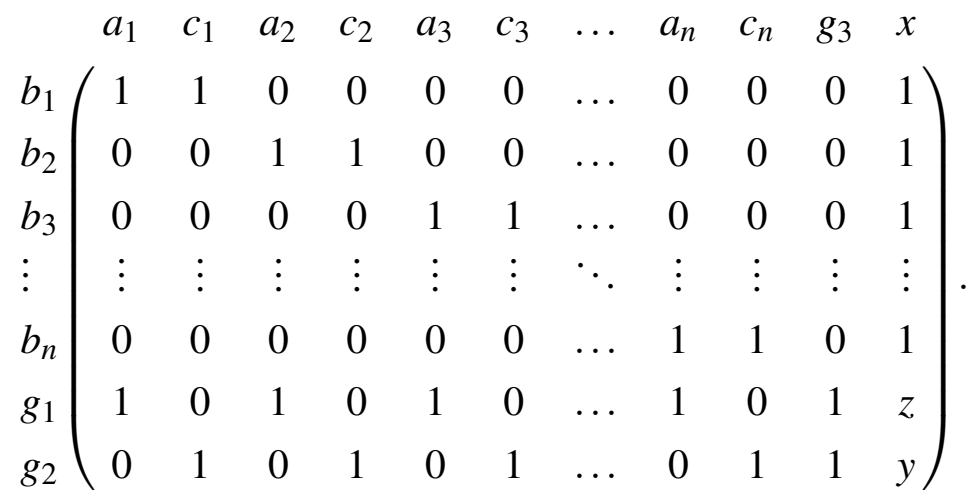


Lemma 5.2.2. If $M$ has an odd number of rows and $x \neq y$, then there is a change of basis so that $M$ has a reduced standard representation matrix $A$ where

$$
A=\left(\begin{array}{ccccccccccc}
1 & 1 & 0 & 0 & 0 & 0 & \ldots & 0 & 0 & 0 & 1 \\
0 & 0 & 1 & 1 & 0 & 0 & \ldots & 0 & 0 & 0 & 1 \\
0 & 0 & 0 & 0 & 1 & 1 & \ldots & 0 & 0 & 0 & 1 \\
\vdots & \vdots & \vdots & \vdots & \vdots & \vdots & \vdots & \vdots & \vdots & \vdots & \vdots \\
0 & 0 & 0 & 0 & 0 & 0 & \ldots & 1 & 1 & 0 & 1 \\
1 & 0 & 1 & 0 & 1 & 0 & \ldots & 1 & 0 & 1 & 0 \\
0 & 1 & 0 & 1 & 0 & 1 & \ldots & 0 & 1 & 1 & 0
\end{array}\right)
$$

Proof. Suppose that $z=1, y=0$. By performing a change of basis so the new basis is $\left\{a_{1}, a_{2}, \ldots, a_{n}, g_{1} . g_{3}\right\}$, we see the required matrix. To see this note that pivoting on $M_{a_{i}, b_{i}}$ for all $i \in\{1, \ldots, n\}$ gives

$$
\begin{aligned}
& \begin{array}{lllllllllll}
b_{1} & c_{1} & b_{2} & c_{2} & b_{3} & c_{3} & \ldots & b_{n} & c_{n} & g_{3} & x
\end{array}
\end{aligned}
$$

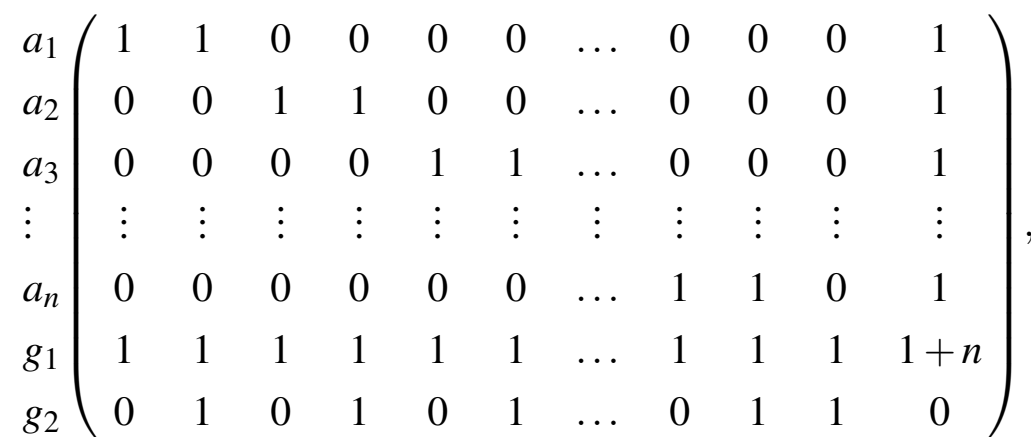

where $n$ is the number of rows of $M$ minus 2. Since $M$ has an odd number of rows $1+n=0$. Pivoting on $M_{g_{2}, g_{3}}$ then gives

$$
\begin{aligned}
& \begin{array}{lllllllllll}
b_{1} & c_{1} & b_{2} & c_{2} & b_{3} & c_{3} & \ldots & b_{n} & c_{n} & g_{2} & x
\end{array} \\
& \begin{array}{l}
a_{1} \\
a_{2} \\
a_{3} \\
\vdots \\
a_{n} \\
g_{1} \\
g_{3}
\end{array}\left(\begin{array}{ccccccccccc}
1 & 1 & 0 & 0 & 0 & 0 & \ldots & 0 & 0 & 0 & 1 \\
0 & 0 & 1 & 1 & 0 & 0 & \ldots & 0 & 0 & 0 & 1 \\
0 & 0 & 0 & 0 & 1 & 1 & \ldots & 0 & 0 & 0 & 1 \\
0 & 0 & 0 & 0 & 0 & 0 & \ldots & 1 & 1 & 0 & 1 \\
1 & 0 & 1 & 0 & 1 & 0 & \ldots & 1 & 0 & 1 & 0 \\
0 & 1 & 0 & 1 & 0 & 1 & \ldots & 0 & 1 & 1 & 0
\end{array}\right),
\end{aligned}
$$

as required. If $z=0, y=1$ the result follows by symmetry. 
Lemma 5.2.3. If $M$ has an even number of rows and has reduced standard representation matrix $A$, where

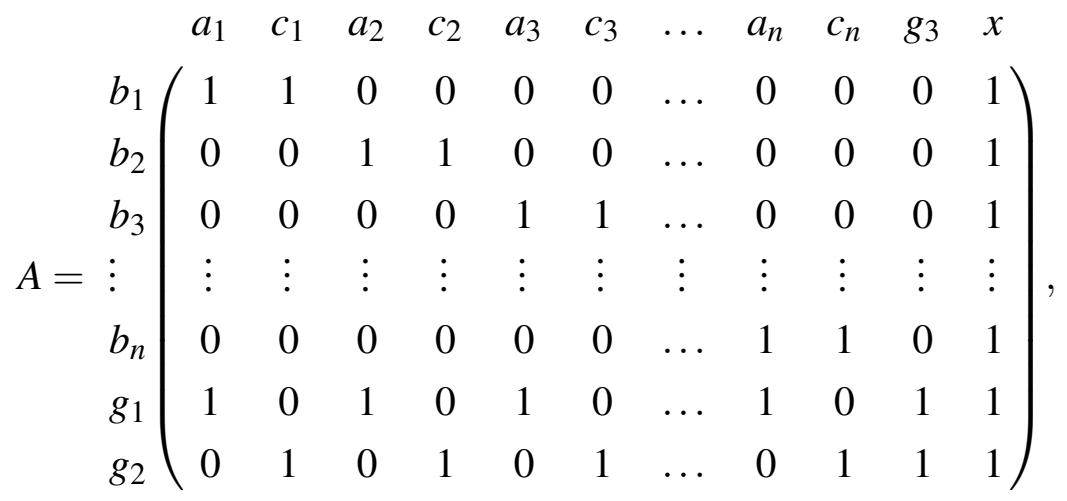

then there is a change of basis so that $M$ is represented by

$$
\left(\begin{array}{ccccccccccc}
1 & 1 & 0 & 0 & 0 & 0 & \ldots & 0 & 0 & 0 & 1 \\
0 & 0 & 1 & 1 & 0 & 0 & \ldots & 0 & 0 & 0 & 1 \\
0 & 0 & 0 & 0 & 1 & 1 & \ldots & 0 & 0 & 0 & 1 \\
\vdots & \vdots & \vdots & \vdots & \vdots & \vdots & \vdots & \vdots & \vdots & \vdots & \vdots \\
0 & 0 & 0 & 0 & 0 & 0 & \ldots & 1 & 1 & 0 & 1 \\
1 & 0 & 1 & 0 & 1 & 0 & \ldots & 1 & 0 & 1 & 0 \\
0 & 1 & 0 & 1 & 0 & 1 & \ldots & 0 & 1 & 1 & 1
\end{array}\right) .
$$

Proof. Perform a change of basis so that the basis is $\left\{a_{1}, a_{2}, \ldots, a_{n}, g_{1}, g_{3}\right\}$. This gives the required matrix.

To see this note that pivoting on $M_{a_{i}, b_{i}}$ for all $i \in\{1, \ldots, n\}$ gives

$$
\begin{aligned}
& \begin{array}{lllllllllll}
b_{1} & c_{1} & b_{2} & c_{2} & b_{3} & c_{3} & \ldots & b_{n} & c_{n} & g_{3} & x
\end{array}
\end{aligned}
$$

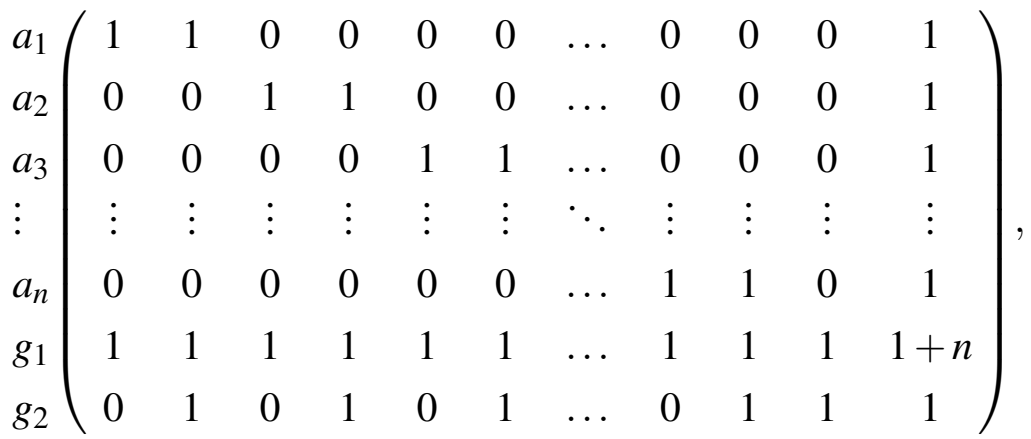

where $n$ is the number of rows of $M$ minus 2. Since $M$ has an even number of 
rows $1+n=1$. Pivoting on $M_{g_{2}, g_{3}}$ then gives

$$
\begin{aligned}
& \begin{array}{lllllllllll}
b_{1} & c_{1} & b_{2} & c_{2} & b_{3} & c_{3} & \ldots & b_{n} & c_{n} & g_{2} & x
\end{array}
\end{aligned}
$$

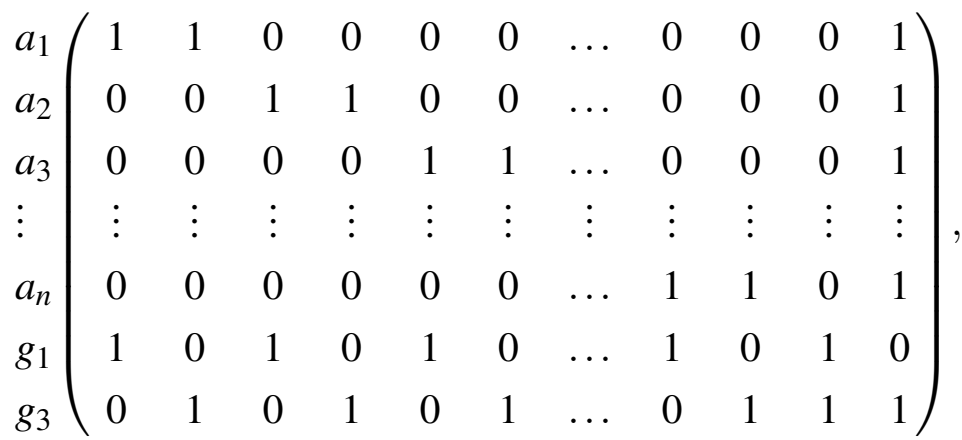

as required.

Lemma 5.2.4. There is a function $[5.2 .4$ such that the following holds. If $n \geq$ $f_{5.2 .4}(t)$, then there is a minor of $M$ of rank at least $t+2$ which has representation

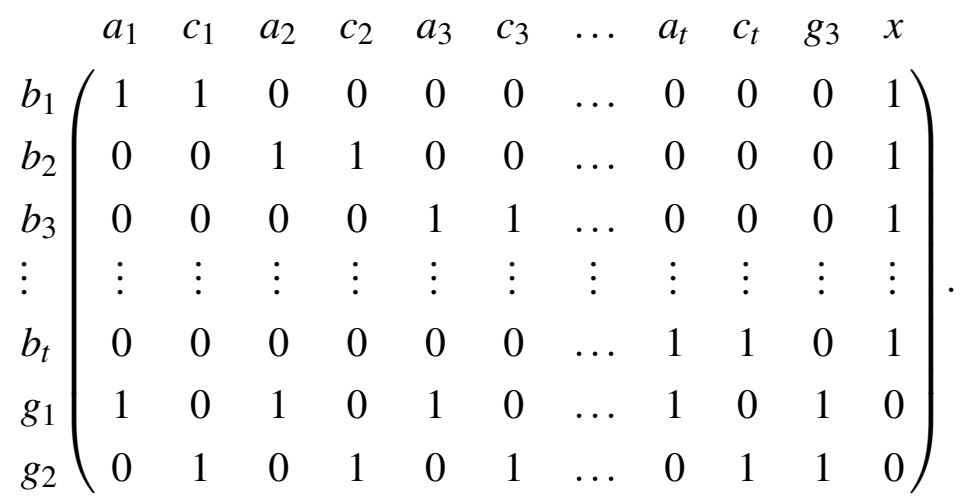

Proof. Let $n=f_{5.2 .4}(t)$. If $M\left(K_{3, n}\right)$ is blocked by a single element, then $M\left(K_{3, n}\right)+x$ can be represented by the following matrix:

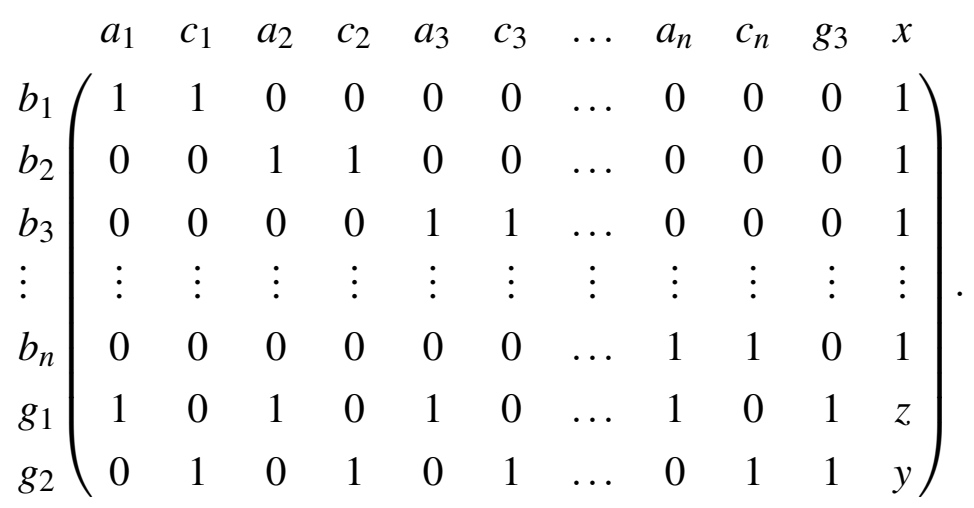

Suppose that $n$ is odd. By Lemma5.2.2, if $0 \in\{z, y\}$ there is a change of basis that 
gives the required matrix. Therefore we assume that $z=y=1$. By contracting $b_{n}$ and deleting $a_{n}, c_{n}$ we get

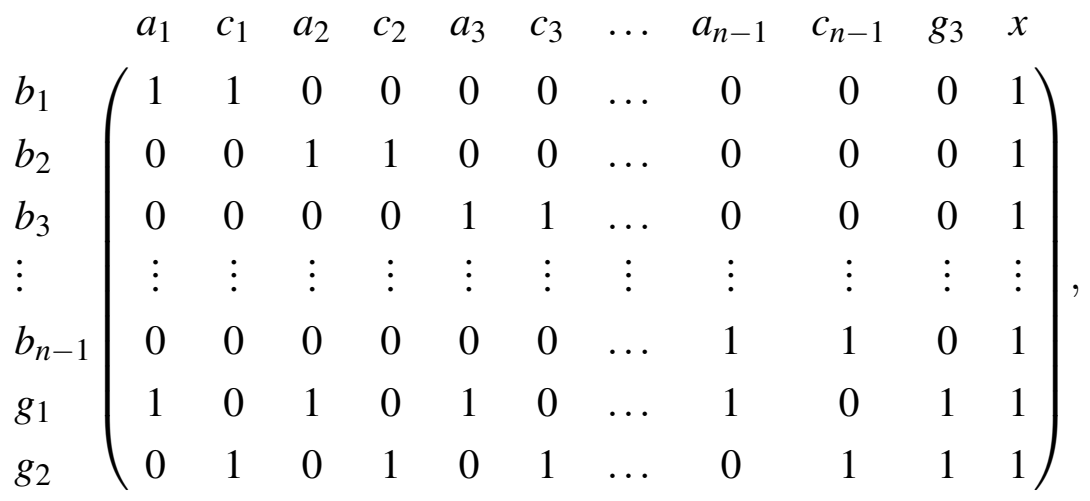

which is a representation of $M\left(K_{3, n-1}\right)$ blocked by a single element. Notice that since $n$ is odd, $n-1$ is even.

Suppose that $n$ is even. By Lemma 5.2.3 there is a change of basis so that $0 \in$ $\{z, y\}$. We may then contract a basis element from some petal and delete the remaining elements of that petal to get $M\left(K_{3, n-1}^{+}\right)$blocked by a single element.

In either case we either have $z=y=0$ or a matroid with rank at least $n$ represented by

$$
\left(\begin{array}{ccccccccc}
1 & 1 & 0 & 0 & \ldots & 0 & 0 & 0 & 1 \\
0 & 0 & 1 & 1 & \ldots & 0 & 0 & 0 & 1 \\
\vdots & \vdots & \vdots & \vdots & \vdots & \vdots & \vdots & & \\
0 & 0 & 0 & 0 & \ldots & 1 & 1 & 0 & 1 \\
1 & 0 & 1 & 0 & \ldots & 1 & 0 & 1 & 0 \\
0 & 1 & 0 & 1 & \ldots & 0 & 1 & 1 & 1
\end{array}\right)
$$

where this matrix has an odd number of rows. By Lemma 5.2.2, there is a minor of $M\left(K_{3, n-2}\right)$ represented by the following rank- $n$ matroid:

$$
\left(\begin{array}{ccccccccc}
1 & 1 & 0 & 0 & \ldots & 0 & 0 & 0 & 1 \\
0 & 0 & 1 & 1 & \ldots & 0 & 0 & 0 & 1 \\
\vdots & \vdots & \vdots & \vdots & \vdots & \vdots & \vdots & & \\
0 & 0 & 0 & 0 & \ldots & 1 & 1 & 0 & 1 \\
1 & 0 & 1 & 0 & \ldots & 1 & 0 & 1 & 0 \\
0 & 1 & 0 & 1 & \ldots & 0 & 1 & 1 & 0
\end{array}\right)
$$


Theorem 5.2.5. Suppose $M$ is matroid such that $M \backslash x \cong M\left(K_{3, n}^{+}\right)$, and every 3 separation of $M \backslash x$ displayed by the canonical flower of $M \backslash x$ is blocked by $x$. If $n \geq 5$ 5.2.4 $(t)$, then $M$ has a $N\left(K_{3, t}\right)$-minor.

Proof. There is a minor of $M$ of rank at least $t+2$ which, after appropriate relabelling can be represented by:

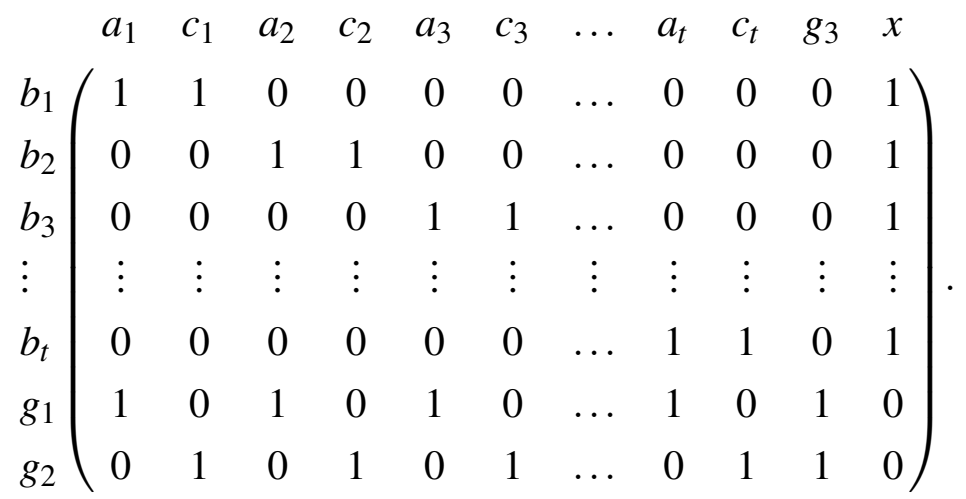

By definition of $N\left(K_{3, n}\right)$ we see that $M / x$ is a representation of $N\left(K_{3, t}\right)$.

\section{Case $\left(b^{\prime}\right)$}

In this case we assume that $\Lambda$ is of $\left(b^{\prime}\right)$ from Lemma 5.2.1. In other words we assume that $M$ has a reduced standard representation matrix

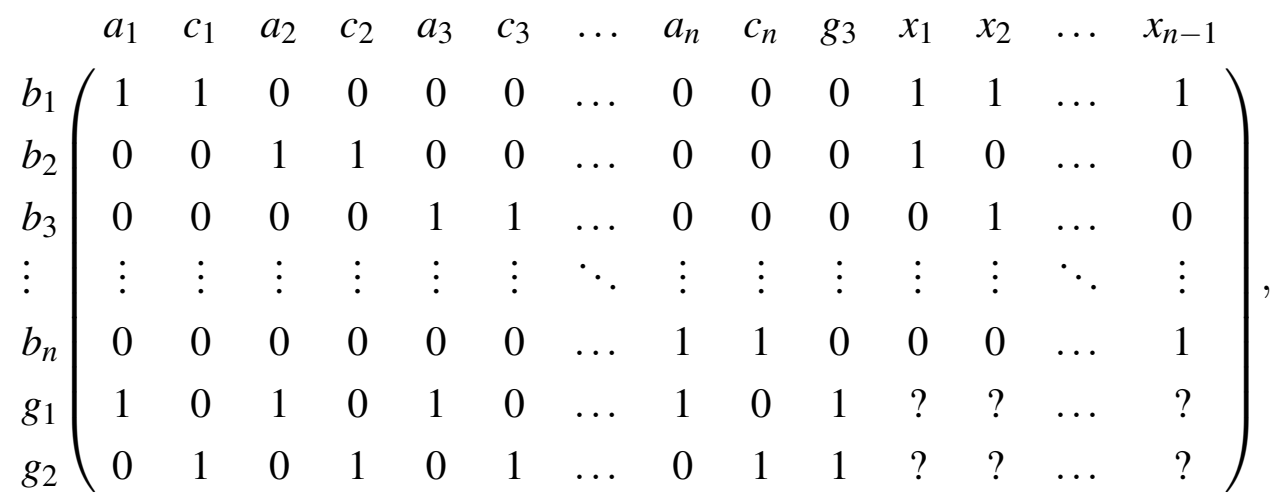

where $x_{1}, \ldots, x_{n-1}$ are the elements of $X$ and $B$, the fixed standard basis for $M \backslash X$, is $\left\{b_{1}, \ldots, b_{n}, g_{1}, g_{2}\right\}$. We show that we can obtain a large minor of $M$ that can be 
represented by the reduced standard representation matrix,

$$
\left(\begin{array}{cccccccccccccc}
1 & 1 & 0 & 0 & 0 & 0 & \ldots & 0 & 0 & 0 & 1 & 1 & \ldots & 1 \\
0 & 0 & 1 & 1 & 0 & 0 & \ldots & 0 & 0 & 0 & 1 & 0 & \ldots & 0 \\
0 & 0 & 0 & 0 & 1 & 1 & \ldots & 0 & 0 & 0 & 0 & 1 & \ldots & 0 \\
\vdots & \vdots & \vdots & \vdots & \vdots & \vdots & \ddots & \vdots & \vdots & \vdots & \vdots & \vdots & \ddots & \vdots \\
0 & 0 & 0 & 0 & 0 & 0 & \ldots & 1 & 1 & 0 & 0 & 0 & \ldots & 1 \\
1 & 0 & 1 & 0 & 1 & 0 & \ldots & 1 & 0 & 1 & 0 & 0 & \ldots & 0 \\
0 & 1 & 0 & 1 & 0 & 1 & \ldots & 0 & 1 & 1 & 0 & 0 & \ldots & 0
\end{array}\right) .
$$

The first step is to show that there is a minor in which the columns labelled by the members of $X$ all have the same two final entries.

Lemma 5.2.6. If $n \geq \sqrt{2.4 .11}(t+1)$, then there is a minor of $M$ of rank-t +2 represented by the following matrix:

$$
\left(\begin{array}{cccccccccccccc}
1 & 1 & 0 & 0 & 0 & 0 & \ldots & 0 & 1 & 1 & 1 & 1 & \ldots & 1 \\
0 & 0 & 1 & 1 & 0 & 0 & \ldots & 0 & 1 & 0 & 0 & 0 & \ldots & 0 \\
0 & 0 & 0 & 0 & 1 & 1 & \ldots & 0 & 0 & 1 & 0 & 0 & \ldots & 0 \\
\vdots & \vdots & \vdots & \vdots & \vdots & \vdots & \ddots & \vdots & \vdots & \vdots & \vdots & \vdots & \ddots & \vdots \\
0 & 0 & 0 & 0 & \ldots & 1 & 1 & 0 & 0 & 0 & 0 & 0 & \ldots & 1 \\
1 & 0 & 1 & 0 & \ldots & 1 & 0 & 1 & x & x & x & x & \ldots & x \\
0 & 1 & 0 & 1 & \ldots & 0 & 1 & 1 & y & y & y & y & \ldots & y
\end{array}\right)
$$

Proof. This follows from Lemma 2.4.11.

Lemma 5.2.7. Suppose $M$ is represented by the following matrix:

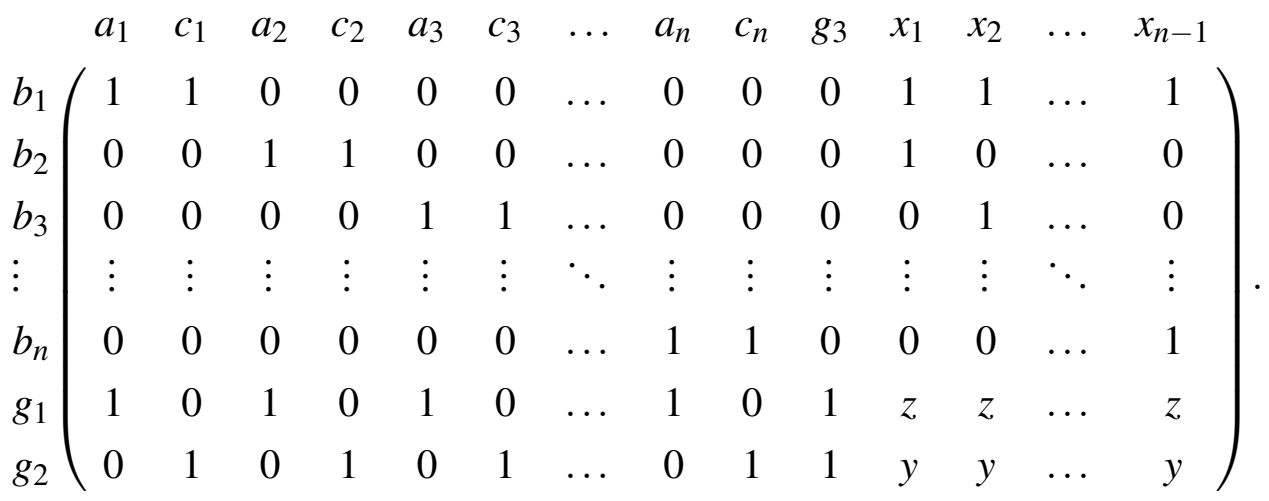

Then there is a function $\int_{5.2 .7}$ such that the following holds. If $n \geq \sqrt{5.2 .7}(t)$, then, 
by relabelling, there is a rank- $(t+2)$-minor of $M$ represented by

$$
\begin{aligned}
& \begin{array}{llllllllllllll}
a_{1} & c_{1} & a_{2} & c_{2} & a_{3} & c_{3} & \ldots & a_{t} & c_{t} & g_{3} & x_{1} & x_{2} & \ldots & x_{t-1}
\end{array} \\
& \begin{array}{l}
b_{1} \\
b_{2} \\
b_{3} \\
\vdots \\
b_{t} \\
g_{1} \\
g_{2}
\end{array}\left(\begin{array}{cccccccccccccc}
1 & 1 & 0 & 0 & 0 & 0 & \ldots & 0 & 0 & 0 & 1 & 1 & \ldots & 1 \\
0 & 0 & 1 & 1 & 0 & 0 & \ldots & 0 & 0 & 0 & 1 & 0 & \ldots & 0 \\
0 & 0 & 0 & 0 & 1 & 1 & \ldots & 0 & 0 & 0 & 0 & 1 & \ldots & 0 \\
0 & 0 & \vdots & \vdots & \vdots & \vdots & \ddots & \vdots & \vdots & \vdots & \vdots & \vdots & \ddots & \vdots \\
1 & 0 & 1 & 0 & 1 & 0 & \ldots & 1 & 0 & 1 & 0 & 0 & \ldots & 0 \\
0 & 1 & 0 & 1 & 0 & 1 & \ldots & 0 & 1 & 1 & 0 & 0 & \ldots & 0
\end{array}\right) .
\end{aligned}
$$

Proof. Let $n=t+3$. First suppose $x=y=1$. Perform a change of basis so that the new basis is $\left\{x_{1}, b_{2}, \ldots, b_{m}, g_{1}, g_{2}\right\}$, then contract $x_{1}$ and delete $a_{1}, c_{1}$ and $b_{1}$. This gives the required matrix. To see this we first pivot on $M_{b_{1}, x_{1}}$ to get

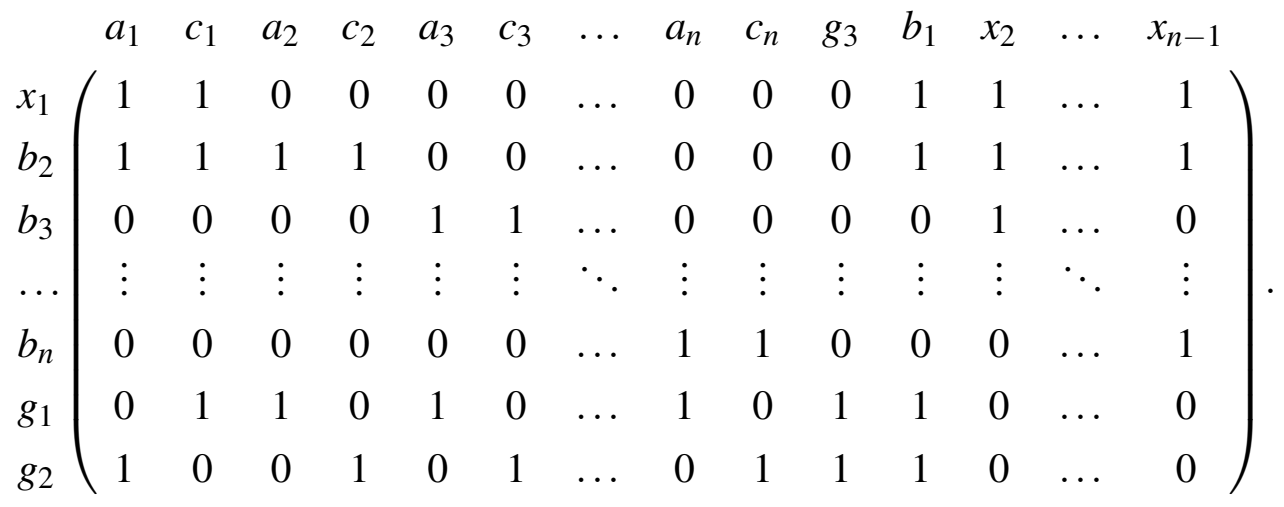

Delete $a_{1}, c_{1}, b_{1}$ and contract $x_{1}$ to get

$$
\begin{aligned}
& a_{2} \\
& b_{2} \\
& b_{3} \\
& \vdots \\
& b_{n} \\
& g_{1} \\
& g_{2}
\end{aligned}\left(\begin{array}{ccccccccccc}
1 & 1 & 0 & 0 & \ldots & 0 & 0 & 0 & 1 & \ldots & 1 \\
0 & 0 & 1 & 1 & \ldots & 0 & 0 & 0 & 1 & \ldots & 0 \\
\vdots & \vdots & \vdots & \vdots & \ddots & \vdots & \vdots & \vdots & \vdots & \ddots & \vdots \\
0 & 0 & 0 & 0 & \ldots & 1 & 1 & 0 & 0 & \ldots & 1 \\
1 & 0 & 1 & 0 & \ldots & 1 & 0 & 1 & 0 & \ldots & 0 \\
0 & 1 & 0 & 1 & \ldots & 0 & 1 & 1 & 0 & \ldots & 0
\end{array}\right),
$$

as required 
Now suppose $z=1, y=0$. Perform a change of basis so that the new basis is $\left\{c_{1}, c_{2}, \ldots, c_{n}, g_{2}, g_{3}\right\}$. This gives the desired matrix. To see this first pivot on $M_{b_{1}, c_{1}}$ to get:

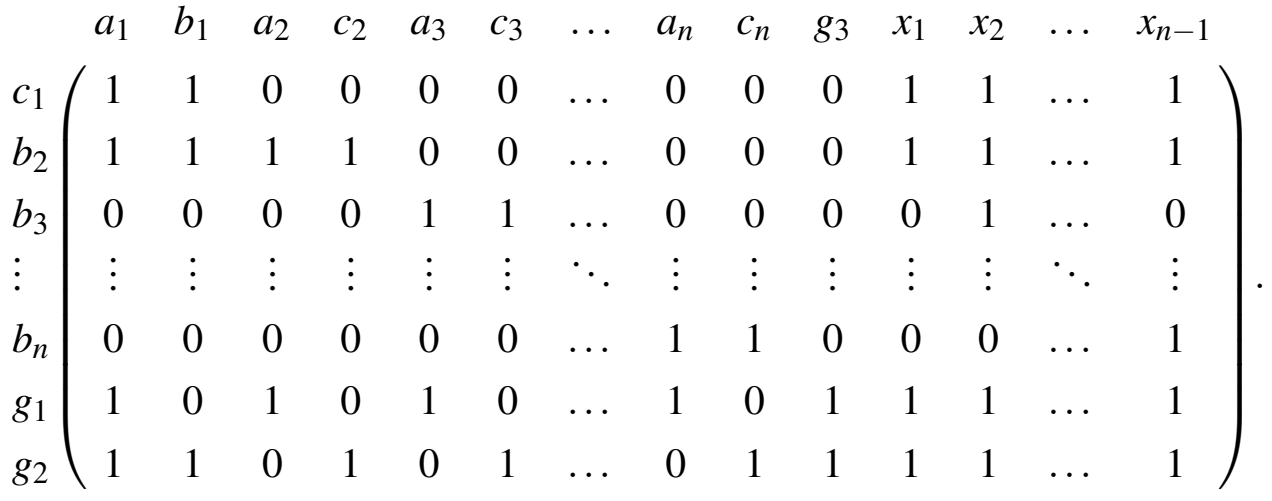

Pivot on $M_{b_{i}, c_{i}}$ for $i \in\{2, \ldots, n\}$ to get

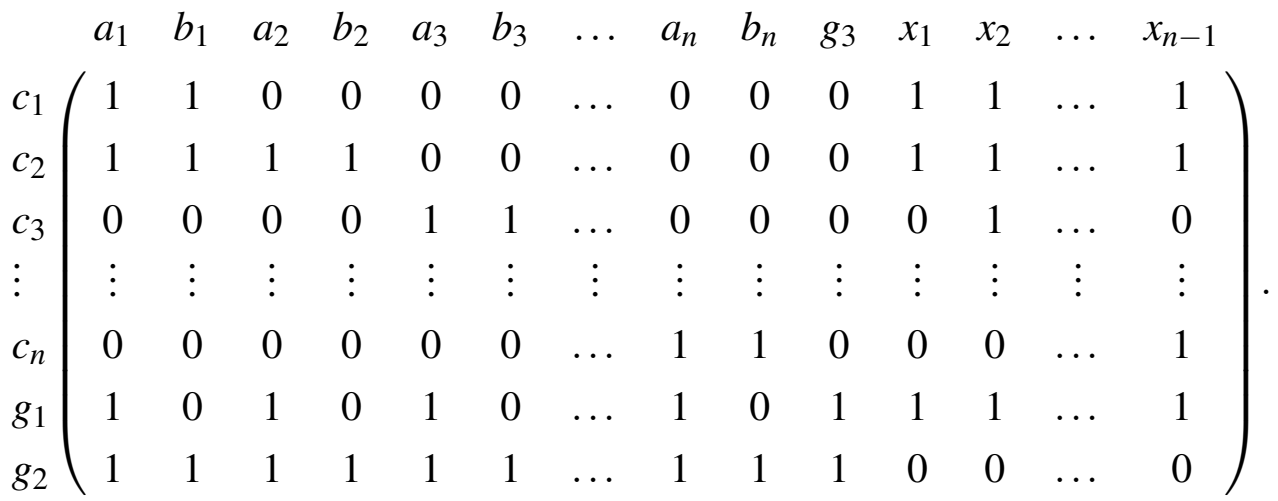

Finally pivot on $M_{g_{1}, g_{3}}$ to get:

$$
\begin{aligned}
& \begin{array}{llllllllllllll}
a_{1} & b_{1} & a_{2} & b_{2} & a_{3} & b_{3} & \ldots & a_{n} & b_{n} & g_{1} & x_{1} & x_{2} & \ldots & x_{n-1}
\end{array}
\end{aligned}
$$

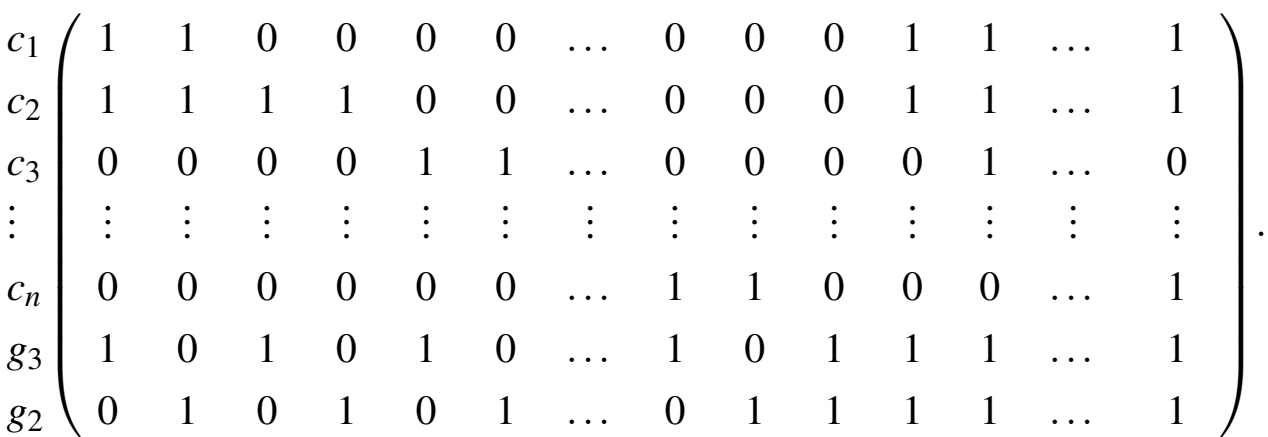


By the arguments above, it is clear that $M$ has a rank- $(t+2)$ minor of the required form.

Lemma 5.2.8. There is a function $\sqrt{5.2 .8}$ such that the following holds. If $M$ is represented by

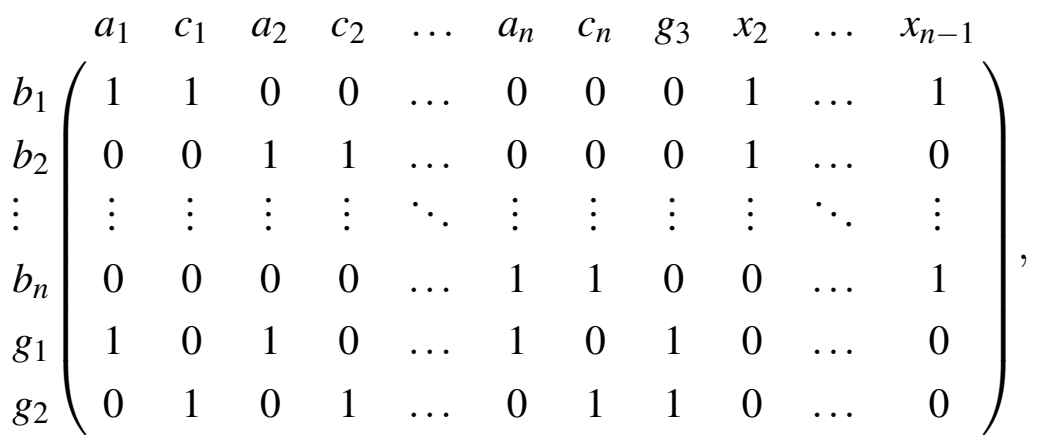

and $n \geq \int\left[5.2 .8(t)\right.$, then $M$ has an $M\left(K_{4, t}\right)$-minor.

Proof. Consider $M \backslash\left\{a_{1}, c_{1}\right\}$. Rearranging the rows and columns of this gives:

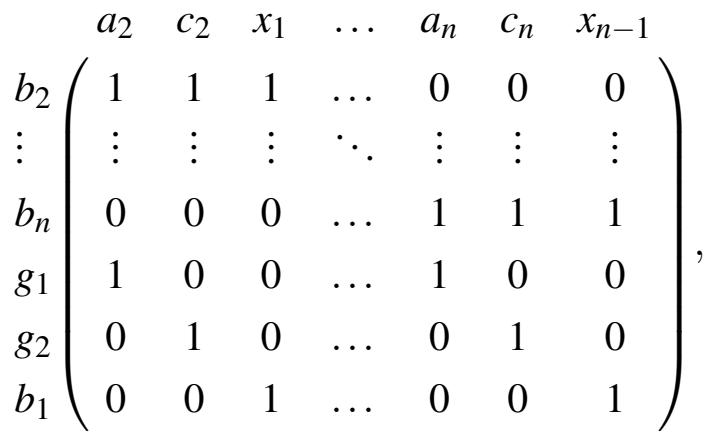

which is a representation of $M\left(K_{4, t}\right)$

We are now in a position to prove the following theorem.

Theorem 5.2.9. Let $M$ be a binary matroid such that $M \backslash X \cong M\left(K_{3, n}^{+}\right)$for some coindependent set $X$ such that $X \subseteq E(M)$. Further suppose that $M$ can be repre- 
sented by a matrix of the following form:

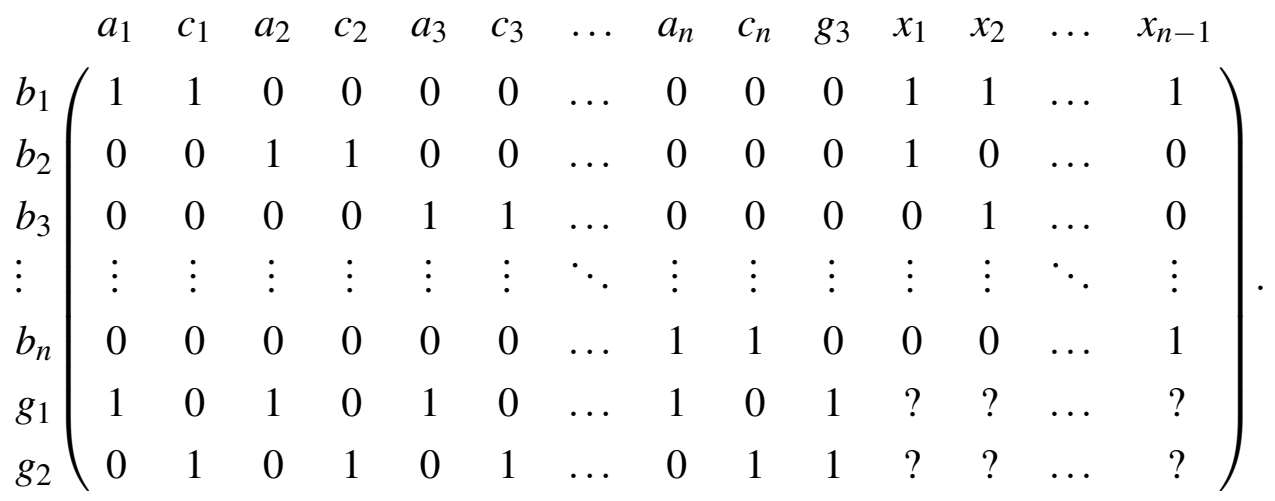

Then, there is a function $\int_{5.2 .9}$ such that if $n \geq \int_{5.2 .9}(t)$, then $M$ has an $M\left(K_{4, t}\right)$ minor.

Proof. Let $n \geq \int\left[2.4 .11\left(\int_{5.2 .7}\left(\int_{5.2 .8}(t)\right)\right)\right.$. By Lemma 5.2.6, there is a rank- $\left(m^{\prime}+2\right)$ minor, $M^{\prime}$, of $M$ represented by

$$
\left(\begin{array}{cccccccccccccc}
1 & 1 & 0 & 0 & 0 & 0 & \ldots & 0 & 1 & 1 & 1 & 1 & \ldots & 1 \\
0 & 0 & 1 & 1 & 0 & 0 & \ldots & 0 & 1 & 0 & 0 & 0 & \ldots & 0 \\
0 & 0 & 0 & 0 & 1 & 1 & \ldots & 0 & 0 & 1 & 0 & 0 & \ldots & 0 \\
\vdots & \vdots & \vdots & \vdots & \vdots & \vdots & \vdots & \vdots & \vdots & \vdots & \vdots & \vdots & \vdots & \vdots \\
0 & 0 & 0 & 0 & \ldots & 1 & 1 & 0 & 0 & 0 & 0 & 0 & \ldots & 1 \\
1 & 0 & 1 & 0 & \ldots & 1 & 0 & 1 & x & x & x & x & \ldots & x \\
0 & 1 & 0 & 1 & \ldots & 0 & 1 & 1 & y & y & y & y & \ldots & y
\end{array}\right)
$$

where $m^{\prime} \geq \int_{5.2 .7}\left(\int_{5.2 .8}(t)\right)$. By Lemma 5.2.7 there is a rank- $m^{\prime \prime}$ minor, $M^{\prime \prime}$, of $M^{\prime}$ represented by

$$
\left(\begin{array}{cccccccccccccc}
1 & 1 & 0 & 0 & 0 & 0 & \ldots & 0 & 0 & 0 & 1 & 1 & \ldots & 1 \\
0 & 0 & 1 & 1 & 0 & 0 & \ldots & 0 & 0 & 0 & 1 & 0 & \ldots & 0 \\
0 & 0 & 0 & 0 & 1 & 1 & \ldots & 0 & 0 & 0 & 0 & 1 & \ldots & 0 \\
\vdots & \vdots & \vdots & \vdots & \vdots & \vdots & \vdots & \vdots & \vdots & \vdots & \vdots & \vdots & \vdots & \vdots \\
0 & 0 & 0 & 0 & 0 & 0 & \ldots & 1 & 1 & 0 & 0 & 0 & \ldots & 1 \\
1 & 0 & 1 & 0 & 1 & 0 & \ldots & 1 & 0 & 1 & 0 & 0 & \ldots & 0 \\
0 & 1 & 0 & 1 & 0 & 1 & \ldots & 0 & 1 & 1 & 0 & 0 & \ldots & 0
\end{array}\right)
$$

where $m^{\prime \prime} \geq \int 5.2 .8(t)$. By Lemma 5.2.8 this means that $M^{\prime \prime}$, and hence $M$, has a 
minor isomorphic to $M\left(K_{4, t}\right)$.

\section{$\operatorname{Case}\left(c^{\prime}\right)$}

Finally consider the case where $\Gamma$ is of form $\left(c^{\prime}\right)$ from Lemma 5.2.1. That is, the case where $M$ be represented by:

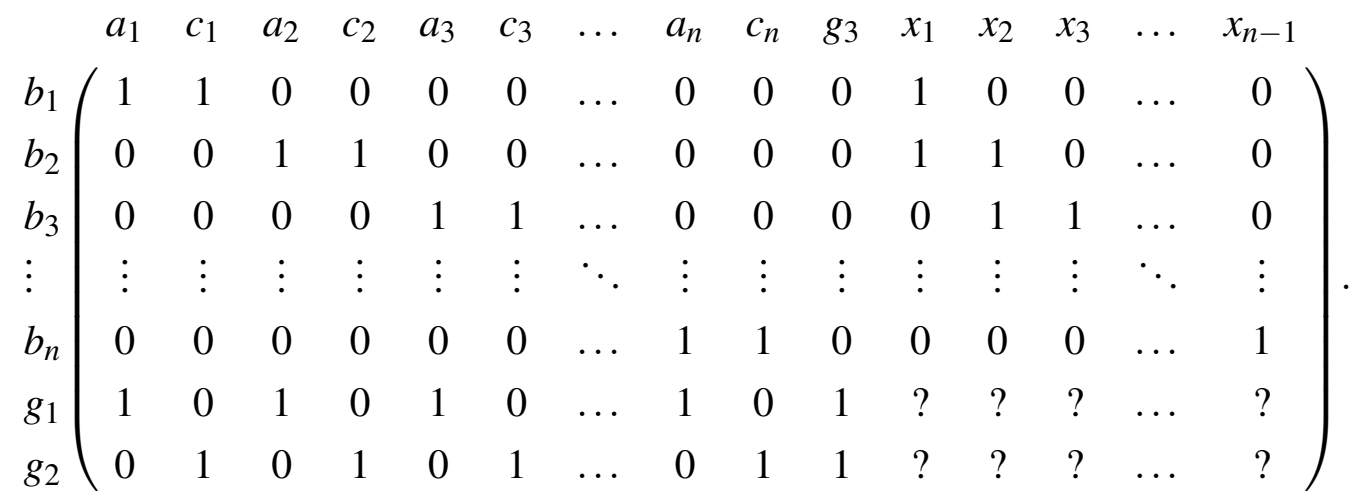

Throughout this case we assume that $M$ can be represented by the above matrix and that $X=\left\{x_{1}, \ldots, x_{n-1}\right\}$.

This is the hardest of the three cases and first we need the following lemma:

Lemma 5.2.10. Let $G$ be a finite group of order $m$. Then there is a function $\sqrt{[5.2 .10}: \mathbb{Z}_{\geq 0} \rightarrow \mathbb{Z}_{\geq 0}$ such that, for any $m$, if $S$ is a string $a_{1}, \ldots, a_{\sqrt[5.2 .10]{ }(t)}$ of group elements, then there is some internal substring of $S$ which can be split into $m$ consecutive sets each of which sum to zero.

Proof. Since $G$ has order $m$ there are $m$ possible values the sum of a sequence of group elements can take. Let $S_{k}=\sum_{i=1}^{k} a_{i}$. If $S$ has length $n$ then there are at least $\frac{n}{m}=p$ integers, $k_{1}, \ldots k_{p}$ such that $S_{k_{1}}=S_{k_{2}}=\cdots=S_{k_{p}}$. Consider $S_{k_{i}}-S_{k_{i-1}}$. It is clear that the sum of $a_{k_{i-1}+1}, \ldots, a_{k_{i}}$ is zero. Therefore $f_{5.2 .10}(t)=m^{2}$ satisfies the requirements of the lemma.

Lemma 5.2.11. There is a function $[5.2 .10$ such that the following holds. If $n \geq$ $\int 5.2 .10(t)$, then there is a rank- $t+2$ minor of $M$ with can be represented by the following reduced standard representation matrix: 


$$
\left(\begin{array}{ccccccccccccccccc}
1 & 1 & 0 & 0 & 0 & 0 & 0 & 0 & \ldots & 0 & 0 & 0 & 1 & 0 & 0 & \ldots & 0 \\
0 & 0 & 1 & 1 & 0 & 0 & 0 & 0 & \ldots & 0 & 0 & 0 & 1 & 1 & 0 & \ldots & 0 \\
0 & 0 & 0 & 0 & 1 & 1 & 0 & 0 & \ldots & 0 & 0 & 0 & 0 & 1 & 1 & \ldots & 0 \\
0 & 0 & 0 & 0 & 0 & 0 & 1 & 1 & \ldots & 0 & 0 & 0 & 0 & 0 & 1 & \ldots & 0 \\
\vdots & \vdots & \vdots & \vdots & \vdots & \vdots & \vdots & \vdots & \ddots & \vdots & \vdots & \vdots & \vdots & \vdots & \vdots & \vdots & \vdots \\
0 & 0 & 0 & 0 & 0 & 0 & 0 & 0 & \ldots & 1 & 1 & 0 & 0 & 0 & 0 & \ldots & 1 \\
1 & 0 & 1 & 0 & 1 & 0 & 1 & 0 & \ldots & 1 & 0 & 1 & 0 & 0 & 0 & \ldots & 0 \\
0 & 1 & 0 & 1 & 0 & 1 & 0 & 1 & \ldots & 0 & 1 & 1 & 0 & 0 & 0 & \ldots & 0
\end{array}\right)
$$

Proof. Every rank-3 petal of $F$ is a 3 -separating triad in $M$. Since $M$ is binary, there is exactly one point in the ambient binary space that is in the span of $P_{i}$ and is not parallel to any element of $M$. Call this element $d_{i}$. Let $\widetilde{M}$ be the matroid obtained by extending $M$ by $\left\{d_{1}, \ldots, d_{n}\right\}$. Clearly $\widetilde{M} \backslash X$ has a paddle partition $\widetilde{F}=\left(\widetilde{P}_{1}, \ldots, \widetilde{P}_{n}, G\right)$ where $\widetilde{P}_{i}=P_{i} \cup d_{i}$ and $G=\left\{g_{1}, g_{2}, g_{3}\right\}$. Since $X \subseteq\langle M \backslash X\rangle$ and for every 3-separation $(A, B)$ of $M$ displayed by $F$ there is some $x \in X$ that blocks $(A, B)$, it follows that $X \subseteq\langle\widetilde{M} \backslash X\rangle$ and for every 3-separation $\left(A^{\prime}, B^{\prime}\right)$ of $\widetilde{M}$ displayed by $\widetilde{F}$ there is some $x \in X$ that blocks $\left(A^{\prime}, B^{\prime}\right)$. Since $P_{i}$ spans $\widetilde{P}_{i}$ for $i \in\{1, \ldots, n\}$ we see that $x \in \operatorname{cl}\left(P_{i}\right)$ if, and only if, $x \in \operatorname{cl}\left(\widetilde{P}_{i}\right)$, and $x \in \operatorname{cl}\left(P_{i} \cup P_{j}\right)$ if, and only if, $x \in \operatorname{cl}\left(\widetilde{P}_{i} \cup \widetilde{P}_{j}\right)$. Therefore, for any $x_{i} \in X$, there does not exist a $j \in\{1, \ldots, n\}$ such that $x_{i} \in \operatorname{cl}\left(\widetilde{P}_{j}\right)$. However, for any $x_{i} \in X$ we see that $x_{i} \in \operatorname{cl}\left(\widetilde{P}_{i} \cup \widetilde{P}_{i+1}\right)$ for $i \in\{1, \ldots, n\}$. Consider some $x_{i} \in X$. For every element $z$ of $\widetilde{P}_{i}$ there is a circuit containing $x_{i}$ and an element of $\widetilde{P}_{i+1}$. Since $\left|C_{1} \triangle C_{2}\right| \geq 2$ for any pair of circuits $C_{1}$ and $C_{2}$, this means that $x_{i}$ induces a matching between $\widetilde{P}_{i}$ and $\widetilde{P}_{i+1}$. Let $x_{i} \in \operatorname{cl}\left(P_{i} \cup P_{i+1}\right)$ and $x_{i+1} \in \operatorname{cl}\left(P_{i+1} \cup P_{i+2}\right)$ and suppose $x_{i}$ matches $\left(e_{1}, e_{2}, e_{3}, e_{4}\right)$ to $\left(e_{1}^{\prime}, e_{2}^{\prime}, e_{3}^{\prime}, e_{4}^{\prime}\right)$, and $x_{i+1}$ matches $\left(e_{1}^{\prime}, e_{2}^{\prime}, e_{3}^{\prime}, e_{4}^{\prime}\right)$ to $\left(e_{1}^{\prime \prime}, e_{2}^{\prime \prime}, e_{3}^{\prime \prime}, e_{4}^{\prime \prime}\right)$ for $e_{j} \in\left\{a_{i}, b_{i}, c_{i}, d_{i}\right\}$ for $e_{j}^{\prime} \in\left\{a_{i+1}, b_{i+1}, c_{i+1}, d_{i+1}\right\}$ and for $e_{j}^{\prime \prime} \in\left\{a_{i+2}, b_{i+2}, c_{i+2}, d_{i+2}\right\}$ for $j \in\{1,2,3,4\}$ and $e_{i} \neq e_{j}, e_{i}^{\prime} \neq e_{j}^{\prime}$ and $e_{i}^{\prime \prime} \neq e_{j}^{\prime \prime}$ if $i \neq j$. When we contract $x_{i+1}$ this gives a matching between the elements of $P_{i}$ and the elements of $P_{i+2}$ and this matching takes $\left(e_{1}, e_{2}, e_{3}, e_{4}\right)$ to $\left(e_{1}^{\prime \prime}, e_{2}^{\prime \prime}, e_{3}^{\prime \prime}, e_{4}^{\prime \prime}\right)$. The matching induces permutations of $(a, b, c, d)$ where $a_{i}$ correspond to $a, b_{i}$ to $b, c_{i}$ to $c$ and $d_{i}$ to $d$ and composition works as described above. By Lemma 5.2.10 there is an internal subset $S$ of $\left(\widetilde{P}_{1}, \ldots, \widetilde{P}_{n}\right)$ such that the following holds.

1. $S$ that can be broken into $t$ sets $S_{1}, \ldots, S_{t}$, where $S_{i}$ is a union of petals and $S_{1}, \ldots, S_{t}$ partition $S$, 
2. for any $S_{i}$ for $i \in\{1, \ldots, t\}$, if $\left.S_{i}=\widetilde{P}_{i}, \ldots, \widetilde{P}_{j}\right)$, then $S_{i}$ is such that when we compose petals to get a matching between $\widetilde{P}_{i}$ and $\widetilde{P}_{j}$ then this matching is the identity matching.

Therefore by deleting $\left\{d_{1}, \ldots, d_{n}\right\}$ we see that $M$ has a minor that can be represented by $M\left(K_{3, n^{\prime}+1}^{+}\right)$augmented by a matrix of the following form

\begin{tabular}{|c|c|c|c|c|c|c|c|c|c|c|c|c|c|}
\hline & $x_{1}$ & $x_{2}$ & $x_{3}$ & $\ldots$ & $x_{a}$ & $x_{a+1}$ & $x_{a+2}$ & $\ldots$ & $x_{a+t}$ & $x_{a+t+1}$ & $x_{a+t+2}$ & $\ldots$ & $x_{n^{\prime}}$ \\
\hline$b_{1}$ & 1 & 0 & 0 & $\ldots$ & 0 & 0 & 0 & $\ldots$ & 0 & 0 & 0 & $\ldots$ & 0 \\
\hline$b_{2}$ & 1 & 1 & 0 & $\ldots$ & 0 & 0 & 0 & $\ldots$ & 0 & 0 & 0 & $\ldots$ & 0 \\
\hline$b_{3}$ & 0 & 1 & 1 & $\ldots$ & 0 & 0 & 0 & $\ldots$ & 0 & 0 & 0 & $\ldots$ & 0 \\
\hline \multirow[t]{2}{*}{$b_{4}$} & 0 & 0 & 1 & $\ldots$ & 0 & 0 & 0 & $\ldots$ & 0 & 0 & 0 & $\ldots$ & 0 \\
\hline & $\vdots$ & $\vdots$ & $\vdots$ & $\vdots$ & $\ddots$ & $\vdots$ & $\vdots$ & $\vdots$ & $\ddots$ & $\vdots$ & $\vdots$ & $\ddots$ & $\vdots$ \\
\hline$b_{a}$ & 0 & 0 & 0 & $\ldots$ & 1 & 0 & 0 & $\ldots$ & 0 & 0 & 0 & $\ldots$ & 0 \\
\hline$b_{a+1}$ & 0 & 0 & 0 & $\ldots$ & 1 & 1 & 0 & $\ldots$ & 0 & 0 & 0 & $\ldots$ & 0 \\
\hline$b_{a+2}$ & 0 & 0 & 0 & $\ldots$ & 0 & 1 & 1 & $\ldots$ & 0 & 0 & 0 & $\ldots$ & 0 \\
\hline \multirow[t]{2}{*}{$b_{a+3}$} & 0 & 0 & 0 & $\ldots$ & 0 & 0 & 1 & $\ldots$ & 0 & 0 & 0 & $\ldots$ & 0 \\
\hline & $\vdots$ & $\vdots$ & $\vdots$ & $\vdots$ & $\ddots$ & $\vdots$ & $\vdots$ & $\vdots$ & $\ddots$ & $\vdots$ & $\vdots$ & $\ddots$ & $\vdots$ \\
\hline$b_{a+t}$ & 0 & 0 & 0 & $\ldots$ & 0 & 0 & 0 & $\ldots$ & 1 & 0 & 0 & $\ldots$ & 0 \\
\hline$b_{a+t+1}$ & 0 & 0 & 0 & $\ldots$ & 0 & 0 & 0 & $\ldots$ & 1 & 1 & 0 & $\ldots$ & 0 \\
\hline$b_{a+t+2}$ & 0 & 0 & 0 & $\ldots$ & 0 & 0 & 0 & $\ldots$ & 0 & 1 & 1 & $\ldots$ & 0 \\
\hline \multirow[t]{2}{*}{$b_{a+t+3}$} & 0 & 0 & 0 & $\ldots$ & 0 & 0 & 0 & $\ldots$ & 0 & 0 & 1 & $\ldots$ & 0 \\
\hline & $\vdots$ & $\vdots$ & $\vdots$ & $\vdots$ & $\ddots$ & $\vdots$ & $\vdots$ & $\vdots$ & $\ddots$ & $\vdots$ & $\vdots$ & $\ddots$ & $\vdots$ \\
\hline$b_{n^{\prime}}$ & 0 & 0 & 0 & 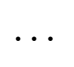 & 0 & 0 & 0 & $\ldots$ & 0 & 0 & 0 & $\ldots$ & 1 \\
\hline$b_{n^{\prime}+1}$ & 0 & 0 & 0 & $\ldots$ & 0 & 0 & 0 & $\ldots$ & 0 & 0 & 0 & $\ldots$ & 1 \\
\hline$g_{1}$ & $?$ & $?$ & $?$ & $\ldots$ & 0 & 0 & 0 & $\ldots$ & $?$ & $?$ & $?$ & $\ldots$ & $?$ \\
\hline$g_{2}$ & $?$ & $?$ & $?$ & $\ldots$ & 0 & 0 & 0 & $\ldots$ & $?$ & $?$ & $?$ & $\ldots$ & $?$ \\
\hline
\end{tabular}

Contract $\left\{b_{1}, \ldots, b_{a}, b_{a+t}, \ldots, b_{n^{\prime}+1}\right\}$ and delete $\left\{w_{1}, \ldots, w_{a}, w_{a+t}, \ldots, w_{n^{\prime}}\right\}$ for all $w \in\{a, c, x\}$ where $a, c$ label the non-basis elements of $F$. This gives the required matrix. 
Lemma 5.2.12. If $M$ is represented by the following rank- $(t+2)$ matrix:

$$
\left(\begin{array}{ccccccccccccccccc}
1 & 1 & 0 & 0 & 0 & 0 & 0 & 0 & \ldots & 0 & 0 & 0 & 1 & 0 & 0 & \ldots & 0 \\
0 & 0 & 1 & 1 & 0 & 0 & 0 & 0 & \ldots & 0 & 0 & 0 & 1 & 1 & 0 & \ldots & 0 \\
0 & 0 & 0 & 0 & 1 & 1 & 0 & 0 & \ldots & 0 & 0 & 0 & 0 & 1 & 1 & \ldots & 0 \\
0 & 0 & 0 & 0 & 0 & 0 & 1 & 1 & \ldots & 0 & 0 & 0 & 0 & 0 & 1 & \ldots & 0 \\
\vdots & \vdots & \vdots & \vdots & \vdots & \vdots & \vdots & \vdots & \ddots & \vdots & \vdots & \vdots & \vdots & \vdots & \vdots & \vdots & \vdots \\
0 & 0 & 0 & 0 & 0 & 0 & 0 & 0 & \ldots & 1 & 1 & 0 & 0 & 0 & 0 & \ldots & 1 \\
1 & 0 & 1 & 0 & 1 & 0 & 1 & 0 & \ldots & 1 & 0 & 1 & 0 & 0 & 0 & \ldots & 0 \\
0 & 1 & 0 & 1 & 0 & 1 & 0 & 1 & \ldots & 0 & 1 & 1 & 0 & 0 & 0 & \ldots & 0
\end{array}\right)
$$

, then $M$ has a rank-t double wheel minor.

Proof. This can be seen from the matrices or by noting that if $M$ is represented by the matrix above, then $M$ is graphic and of the following form:

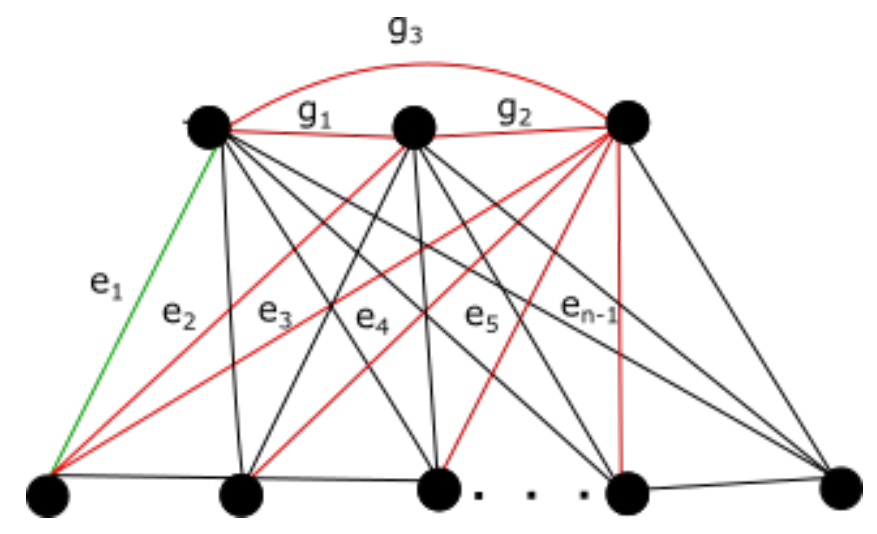

From this we can see that $M \backslash\left\{e_{2}, \ldots, e_{n-1}, g_{1}, g_{2}, g_{3}\right\} /\left\{e_{1}, e_{n}\right\}$ is a double wheel.

Tying the lemmas in this case together we get the following theorem.

Theorem 5.2.13. There is a function $\sqrt{5.2 .13}$ such that the following holds. If $M$ is 
binary matroid such that $M \backslash X \cong M\left(K_{3, n}\right)$ and $M$ can be represented by:

\begin{tabular}{|c|c|c|c|c|c|c|c|c|c|c|c|c|c|c|c|c|c|}
\hline & $a_{1}$ & $c_{1}$ & $a_{2}$ & $c_{2}$ & $a_{3}$ & $c_{3}$ & $\ldots$ & $a_{n-1}$ & $c_{n-1}$ & $a_{n}$ & $c_{n}$ & $g_{3}$ & $x_{1}$ & $x_{2}$ & $x_{3}$ & $\ldots$ & $x_{n-1}$ \\
\hline$b_{1}$ & 1 & 1 & 0 & 0 & 0 & 0 & . & 0 & 0 & 0 & 0 & 0 & 1 & 0 & 0 & $\ldots$ & 0 \\
\hline$b_{2}$ & 0 & 0 & 1 & 1 & 0 & 0 & $\ldots$ & 0 & 0 & 0 & 0 & 0 & 1 & 1 & 0 & $\ldots$ & 0 \\
\hline$b_{3}$ & 0 & 0 & 0 & 0 & 1 & 1 & $\ldots$ & 0 & 0 & 0 & 0 & 0 & 0 & 1 & 1 & $\ldots$ & 0 \\
\hline : & $\vdots$ & $\vdots$ & $\vdots$ & $\vdots$ & $\vdots$ & $\vdots$ & $\vdots$ & $\vdots$ & $\vdots$ & $\vdots$ & $\vdots$ & $\vdots$ & $\vdots$ & $\vdots$ & $\vdots$ & $\vdots$ & $\vdots$ \\
\hline$b_{n-1}$ & 0 & 0 & 0 & 0 & 0 & 0 & & 1 & 1 & 0 & 0 & 0 & 0 & 0 & 0 & $\ldots$ & 1 \\
\hline$b_{n}$ & 0 & 0 & 0 & 0 & 0 & 0 & . & 0 & 0 & 1 & 1 & 0 & 0 & 0 & $\ldots$ & 1 & \\
\hline$g_{1}$ & 1 & 0 & 1 & 0 & 1 & 0 & $\ldots$ & 1 & 0 & 1 & 0 & 1 & $?$ & $?$ & $?$ & $\ldots$ & $?$ \\
\hline$g_{2}$ & 0 & 1 & 0 & 1 & 0 & 1 & $\ldots$ & 0 & 1 & 0 & 1 & 1 & $?$ & $?$ & $?$ & & $?$ \\
\hline
\end{tabular}

where $n \geq \sqrt{5.2 .13}(t)$, then $M$ has a double wheel of rank at least $t$ as a minor.

Proof. Let $\int_{5.2 .13}(t)=\int_{5.2 .10}(t)$. By Lemma 5.2.11 $M$ has a rank- $(t+2)$ minor, $M^{\prime}$, with a reduced standard representation

$$
\left(\begin{array}{ccccccccccccccccc}
1 & 1 & 0 & 0 & 0 & 0 & 0 & 0 & \ldots & 0 & 0 & 0 & 1 & 0 & 0 & \ldots & 0 \\
0 & 0 & 1 & 1 & 0 & 0 & 0 & 0 & \ldots & 0 & 0 & 0 & 1 & 1 & 0 & \ldots & 0 \\
0 & 0 & 0 & 0 & 1 & 1 & 0 & 0 & \ldots & 0 & 0 & 0 & 0 & 1 & 1 & \ldots & 0 \\
0 & 0 & 0 & 0 & 0 & 0 & 1 & 1 & \ldots & 0 & 0 & 0 & 0 & 0 & 1 & \ldots & 0 \\
\vdots & \vdots & \vdots & \vdots & \vdots & \vdots & \vdots & \vdots & \ddots & \vdots & \vdots & \vdots & \vdots & \vdots & \vdots & \vdots & \vdots \\
0 & 0 & 0 & 0 & 0 & 0 & 0 & 0 & \ldots & 1 & 1 & 0 & 0 & 0 & 0 & \ldots & 1 \\
1 & 0 & 1 & 0 & 1 & 0 & 1 & 0 & \ldots & 1 & 0 & 1 & 0 & 0 & 0 & \ldots & 0 \\
0 & 1 & 0 & 1 & 0 & 1 & 0 & 1 & \ldots & 0 & 1 & 1 & 0 & 0 & 0 & \ldots & 0
\end{array}\right)
$$

. By Lemma 5.2.12, $M^{\prime}$, and hence $M$, has a rank- $t$ double wheel minor.

\subsection{Blocking a Paddle}

In this section we focus on blocking the 3-separations displayed by $F$ in $M$ when $F$ is a general binary paddle. Before we do this we set up the following hypotheses for this section:

- $M$ is a binary matroid. 
- The partition $F=\left(P_{1}, \ldots, P_{n}, G\right)$ of $E(M)-X$ is a paddle of $M \backslash X$ where $X$ a coindependent set.

- $G$ is a guts petal of $F$ and $\left\{P_{1}, \ldots, P_{n}\right\}$ are proper petals of $F$.

- $G$ contains two points, $\left\{g_{1}, g_{2}\right\}$.

- There is no partition $\left(P_{1}, \ldots, P_{i}^{\prime}, P_{i}^{\prime \prime}, \ldots, P_{n}, G\right)$ that is a paddle.

- The elements of $X$ are a minimal set of blocking elements for the displayed 3-separations of $M$.

- The set $B$ is a basis of $M$ containing two elements $\left\{g_{1}, g_{2}\right\} \subseteq G$ for every $i \in\{1, \ldots, n\}$, there is some element of $P_{i} \in B$.

- For $x \in X$, let $C_{x}$ denote the fundamental circuit of $x$ with respect to $B$.

Lemma 5.1.2 is restated below.

Lemma 5.1.2. If $n \geq \int 2.4 .9(t)$, then there is a minor $M^{\prime}$ of $M$ such that $M^{\prime} \backslash$ $\left(X \cap E\left(M^{\prime}\right)\right)$ has a flower $F^{\prime} \subseteq F$ with at least $t+1$ petals which, after possible relabelling, can be represented by one of the following matrices:

$$
\left(\begin{array}{cccccc}
P_{1}^{\prime} & 0 & 0 & \ldots & 0 & Q_{1} \\
0 & P_{2}^{\prime} & 0 & \ldots & 0 & Q_{2} \\
0 & 0 & P_{3}^{\prime} & \ldots & 0 & Q_{3} \\
\vdots & \vdots & \vdots & \ddots & \vdots & \vdots \\
0 & 0 & 0 & \ldots & P_{t}^{\prime} & Q_{n} \\
G_{1} & G_{2} & G_{3} & \ldots & G_{t} & ?
\end{array}\right)
$$

(a)

$$
\left(\begin{array}{ccccccccc}
P_{1}^{\prime} & 0 & 0 & \ldots & 0 & Q_{1} & Q_{2} & \ldots & Q_{n} \\
0 & P_{2}^{\prime} & 0 & \ldots & 0 & Q_{1}^{\prime} & 0 & \ldots & 0 \\
0 & 0 & P_{3}^{\prime} & \ldots & 0 & 0 & Q_{2}^{\prime} & \ldots & 0 \\
\vdots & \vdots & \vdots & \ddots & \vdots & \vdots & \vdots & \ddots & \vdots \\
0 & 0 & 0 & \ldots & P_{t}^{\prime} & 0 & 0 & \ldots & Q_{n}^{\prime} \\
G_{1} & G_{2} & G_{3} & \ldots & G_{t} & ? & ? & \ldots & ?
\end{array}\right)
$$




$$
\left(\begin{array}{cccccccccc}
P_{1}^{\prime} & 0 & 0 & \ldots & 0 & 0 & Q_{1} & 0 & \ldots & 0 \\
0 & P_{2}^{\prime} & 0 & \ldots & 0 & 0 & Q_{1}^{\prime} & Q_{2} & \ldots & 0 \\
0 & 0 & P_{3}^{\prime} & \ldots & 0 & 0 & 0 & Q_{2}^{\prime} & \ldots & 0 \\
\vdots & \vdots & \vdots & \ddots & \vdots & \vdots & \vdots & \vdots & \ddots & \vdots \\
0 & 0 & 0 & \ldots & P_{n-1}^{\prime} & 0 & 0 & 0 & \ldots & Q_{n-1} \\
0 & 0 & 0 & \ldots & 0 & P_{n}^{\prime} & 0 & 0 & \ldots & Q_{n-1}^{\prime} \\
G_{1} & G_{2} & G_{3} & \ldots & G_{t-1} & G_{t} & ? & ? & \ldots & ?
\end{array}\right)
$$

Where, for $i \in\{1, \ldots, n\}$ the submatrices $P_{i}^{\prime}, Q_{i}$ and $G_{i}$ are matrices such that the following hold.

i) $G_{i}$ has two rows and the rows of $G_{i}$ are labelled by the elements in the guts petal of $P$,

ii) $G_{1}, \ldots, G_{n}$ represent matrices that each contain at least one non-zero entry in every row,

iii) the rows of $P_{i}^{\prime}$ are labelled by the basis elements of $\left(M \mid P_{i}\right)$,

iv) the columns containing columns of $P_{i}^{\prime}$ label the elements of $P_{i}-B$, and

v) $Q_{i}, Q_{i}^{\prime}$ represent $\left(\left|r\left(P_{i}\right)\right|-2\right) \times 1$ matrices with rows labelled by the basis elements of $P_{i}$ and for every $i \in\{1, \ldots, n\}$ there is a 1 in some row of $Q_{i}$.

We now split the analysis into three cases, one for each of the matrices above.

\subsubsection{Case $(a)$}

We now consider the case where $\Lambda$ is of form $(a)$ from Lemma 5.1.2. This means that $F$ is blocked by a single element, in other words $M$ can be represented by the reduced standard representation matrix

$$
\left(\begin{array}{cccccc}
P_{1}^{\prime} & 0 & 0 & \ldots & 0 & Q_{1} \\
0 & P_{2}^{\prime} & 0 & \ldots & 0 & Q_{2} \\
0 & 0 & P_{3}^{\prime} & \ldots & 0 & Q_{3} \\
\vdots & \vdots & \vdots & \ddots & \vdots & \vdots \\
0 & 0 & 0 & \ldots & P_{t}^{\prime} & Q_{n} \\
G_{1} & G_{2} & G_{3} & \ldots & G_{t} & ?
\end{array}\right)
$$


where, for $i \in\{1, \ldots, n\}$, the submatrices $P_{i}^{\prime}, Q_{i}$ and $G_{i}$ are such that the following hold.

1. $G_{i}$ has two rows and the rows of $G_{i}$ are labelled by the elements in the guts petal of $P$,

2. $G_{1}, \ldots, G_{n}$ represent matrices that each contain at least one non-zero entry in every row,

3. the rows of $P_{i}^{\prime}$ are labelled by the basis elements of $\left(M \mid P_{i}\right)$,

4. the columns containing columns of $P_{i}^{\prime}$ label the elements of $P_{i}-B$, and

5. $Q_{i}, Q_{i}^{\prime}$ represent $\left(\left|r\left(P_{i}\right)\right|-2\right) \times 1$ matrices with rows labelled by the basis elements of $P_{i}$ and, for every $i \in\{1, \ldots, n\}$, there is a 1 in some row of $Q_{i}$.

Lemma 5.3.1. The matroid $M \backslash x$ has a minor $M^{\prime}$ such that $\left.M^{\prime} \backslash x \cong M_{(} K_{3, n}^{+}\right)$and every 3-separation displayed by the canonical flower of $M^{\prime} \backslash x$ is blocked by $x$.

Proof. This follows immediately from Lemma 2.3.9.

This reduces the case where $\Lambda$ is of form $(a)$ of Lemma 5.1.2 to the case where $\Lambda$ is of from $\left(a^{\prime}\right)$ of Lemma 5.2.1, that is we have reduced the case of blocking a paddle with a single element to the case of blocking $K_{3, n}$ with a single element. Thus, as an immediate corollary of Lemma 5.3.1 combined with Lemma 5.2.4. we get the following theorem.

Theorem 5.3.2. Let $M$ be a binary matroid with an element $x$ such that the following hold. The matroid $M \backslash x$ has a paddle $F$ with at least $n \geq \sqrt{5.2 .4}(t)$ petals, $x \in \operatorname{cl}(E(M)-x)$, and $x$ blocks every displayed 3-separation in $F$. Then $M$ has a $N\left(K_{3, n}\right)$-minor. 
Case $(b)$

Consider the matrix $(b)$ from Lemma 5.1.2, that is the matrix given below.

$$
\left(\begin{array}{ccccccccc}
P_{1}^{\prime} & 0 & 0 & \ldots & 0 & Q_{1} & Q_{2} & \ldots & Q_{n} \\
0 & P_{2}^{\prime} & 0 & \ldots & 0 & Q_{1}^{\prime} & 0 & \ldots & 0 \\
0 & 0 & P_{3}^{\prime} & \ldots & 0 & 0 & Q_{2}^{\prime} & \ldots & 0 \\
\vdots & \vdots & \vdots & \ddots & \vdots & \vdots & \vdots & \ddots & \vdots \\
0 & 0 & 0 & \ldots & P_{n}^{\prime} & 0 & 0 & \ldots & Q_{n}^{\prime} \\
G_{1} & G_{2} & G_{3} & \ldots & G_{n} & ? & ? & \ldots & ?
\end{array}\right)
$$

where, for $i \in\{1, \ldots, n\}, P_{i}^{\prime}, Q_{i}$ and $G_{i}$ are matrices where

i) $G_{i}$ has two rows and the rows of $G_{i}$ are labelled by the elements in the guts petal of $P$,

ii) $G_{1}, \ldots, G_{n}$ represent matrices that each contain at least one non-zero entry in every row,

iii) the rows of $P_{i}^{\prime}$ are labelled by the basis elements of $\left(M \mid P_{i}\right)$,

iv) the columns containing columns of $P_{i}^{\prime}$ label the elements of $P_{i}-B$, and

v) $Q_{i}, Q_{i}^{\prime}$ represent $\left(\left|r\left(P_{i}\right)\right|-2\right) \times 1$ matrices with rows labelled by the basis elements of $P_{i}$ and for every $i \in\{1, \ldots, n\}$ there is a 1 in some row of $Q_{i}$.

In this case assume that $M$ is represented by the matrix above, the elements of $G$ are the elements labelling the rows of $G_{i}$ for $i \in\{1, \ldots, n\}$ and for $i \in\{1, \ldots, n\}$ the elements of $P_{i}$ are the elements labelling the rows and columns of $P_{i}^{\prime}$.

Lemma 5.3.3. There is a minor $M^{\prime}$ of $M$ such that the following hold.

i) The matroid $M^{\prime} \backslash X$ has a flower $F^{\prime}=\left(P_{1}, P_{2}^{\prime}, \ldots, P_{n}^{\prime}\right)$, where $P_{i}^{\prime} \subseteq P_{i}$ for $i \in$ $\{2, \ldots, n\}$,

ii) All petals of $F^{\prime}$ but $P_{1}$ are 3-separating triads,

iii) For every 3-separation of $M^{\prime}$ displayed by $F^{\prime}$, there is is an element $x \in X$ that blocks this separation, 
iv) for all $x_{i} \in X$ there is a unique $P_{i}$ such that $x_{i}$ blocks $\left(P_{1} \cup P_{i}, E\left(M^{\prime} \backslash X\right)-\right.$ $\left.\left(P_{1} \cup P_{i}\right)\right)$ and $x_{i} \in \mathrm{cl}_{M^{\prime}}\left(P_{1} \cup P_{i}\right)$, and

v) $X$ is a minimal blocking set for the 3-separations of $M^{\prime} \backslash X$ displayed by $F^{\prime}$.

Proof. Since all petals except $P_{1}$ contain a representative of exactly one blocking element, this follows from Lemma 2.3.9.

We now look at reducing the size of $P_{1}$ while still keeping the property that every displayed 3-separation is blocked.

Lemma 5.3.4. There is a minor $M^{\prime}$ of $M$ such that the following hold.

i) The matroid $M^{\prime} \backslash X$ has a paddle $F^{\prime}=\left(P_{1}^{\prime}, \ldots, P_{n}^{\prime}\right)$ with the property that $M^{\prime} \mid\left(P_{1}^{\prime} \cup\left\{g_{1}, g_{2}\right\}\right) / e$ is connected for any $e \in P_{1}^{\prime}$,

ii) $X \subseteq E\left(M^{\prime}\right)$,

iii) and every 3-separation in $M^{\prime}$ displayed by $F^{\prime}$ is blocked by an element of $X$ and $X$ in minimal with respect to this.

Proof. Since $M \mid\left(P_{1} \cup\left\{g_{1}, g_{2}\right\}\right)$ is connected, for every $e \in P_{1}$ either $M \mid\left(P_{1} \cup\right.$ $\left.\left\{g_{1}, g_{2}\right\}\right) \backslash\{e\}$ is connected, $M \mid\left(P_{1} \cup\left\{g_{1}, g_{2}\right\}\right) /\{e\}$ is connected. An element $x_{i} \in X$ blocks $P_{i}$ if, and only if, $x_{i} \notin \operatorname{cl}\left(P_{1}\right)$ and $x_{i} \notin \operatorname{cl}\left(P_{i}\right)$ and $x_{i} \in \operatorname{cl}\left(P_{1} \cup P_{i}\right)$. Therefore we may, without unblocking any petals of $F$, delete any element of $P_{1}$ that is such that $M \mid\left(\left(P_{1}-e\right) \cup\left\{g_{1}, g_{2}\right\}\right)$ is connected and $r\left(P_{1}-e\right)=r\left(P_{1}\right)$. If $r\left(P_{1}-e\right)=r\left(P_{1}\right)$ then $e$ is a coloop in $M \mid\left(P_{1} \cup\left\{g_{1}, g_{2}\right\}\right)$ so $M \mid\left(P_{1} \cup\left\{g_{1}, g_{2}\right\}\right) / e$ is connected. Inductively this means that we can find a minor $M^{\prime}$ of $M$ such that the following hold.

i) $M^{\prime} \backslash X$ has a paddle $F^{\prime}=\left(P_{1}^{\prime}, \ldots, P_{n}^{\prime}\right)$ with the property that $M^{\prime} \mid\left(P_{1}^{\prime} \cup\right.$ $\left.\left\{g_{1}, g_{2}\right\}\right) / e$ is connected for any $e \in P_{1}^{\prime}$,

ii) $X \subseteq E\left(M^{\prime}\right)$, and

iii) every 3-separation in $M^{\prime}$ displayed by $F^{\prime}$ is blocked by an element of $X$ and $X$ in minimal with respect to this. 
Consider a petal $P_{1}^{\prime}$ as described in the lemma above. For every $x_{i} \in X$ we know that $x_{i} \in \operatorname{cl}\left(P_{1} \cup P_{i}\right)$. Let $A_{i}$ denote the subset of $P_{1}^{\prime} \cap B$ such that $x_{i} \in \operatorname{cl}\left(A_{i} \cup P_{i}\right)$. Suppose there were some $e \in P_{1}^{\prime}$ that was not, for some $i$, contained in $A_{i}$. Contract this element. Now assume that every element $e$ of $P_{1}^{\prime}$ is contained in $A_{i}$ for some $i$. We wish to contract all but one element of each $A_{i}$, which, for a single $A_{i}$ at a time, we are able to do since, if $x \in\left\langle\left(\left\{a_{1}, \ldots, a_{n}\right\}\right)\right\rangle$, then $x \in \operatorname{cl}_{\left(M / a_{i}\right)+x}\left(\left\{a_{2}, \ldots, a_{n}\right\}\right)$. However we may run across a problem that we cannot contract all but one element of some $A_{i}$ without causing a problem with $A_{j}$ for some $j$. For example we have a problem if $A_{1}=\left\{a_{2}, a_{3}\right\}, A_{2}=\left\{a_{2}\right\}$ and $A_{3}=\left\{a_{3}\right\}$.

Lemma 2.4.10 is useful in solving this problem.

Lemma 5.3.5. Let $M$ be such that $M \backslash X$ has a paddle $F=\left(P_{1}, \ldots, P_{n}\right)$ with the following properties.

I) Every element e in $P_{1}$ is such that $M \mid\left(P_{1} \cup\left\{g_{1}, g_{2}\right\}\right) / e$ is connected,

II) $n \geq \int 2.4 .10(t)$,

III) No element of $P_{1}$ can be contracted without unblocking some 3-separation of $M$ displayed by $F$.

Then there is a minor, $M^{\prime}$, of $M$ such that, for coindependent set $X^{\prime}=X \cap E\left(M^{\prime}\right)$, the matroid $M^{\prime} \backslash X^{\prime}$ has a flower $F^{\prime}=\left(P_{1}^{\prime}, \ldots, P_{t}^{\prime}\right)$ with at least $t$ petals such that, after possible relabelling, one of the following holds.

i) For all $P_{j}$ where $j \in\{1, \ldots, t\}, P_{j}$ is a 3 -separating triad,

ii) there is some $a \in P_{1}^{\prime}$ such that for every $x \in X$ there exists $i \in\{2, \ldots, t\}$ such that $x \in \operatorname{cl}\left(a \cup P_{i}^{\prime}\right)$, or

iii) for every $x_{i} \in X$ there exists some $i \in\{2, \ldots, t\}$ such that $x \in \operatorname{cl}\left(p_{i} \cup P_{i}^{\prime}\right)$ where $p_{i} \in P_{1}$. Moreover, for $i, j \in\{2, \ldots, t\}$, if $i \neq j$, then $p_{i} \neq p_{j}$ and $P_{i} \neq P_{i}^{\prime}$.

Proof. As before, let $A_{i}$ denote the subset of $P_{1}^{\prime} \cap B$ such that $x_{i} \in \operatorname{cl}\left(A_{i} \cup P_{i}\right)$. Consider a matrix, $\Upsilon$, with rows labelled by $P_{2}, \ldots, P_{n}$ and columns labelled by the elements of $P_{1}$. Construct the $\Upsilon$ as follows:

$$
\Upsilon_{P_{i}, a_{j}}=\left\{\begin{array}{l}
1 \text { if } a_{j} \text { is contained in } A_{i} \\
0 \text { otherwise }
\end{array}\right.
$$


The matrix $\Upsilon$ has at least one 1 in every row and every column so, by Lemma 2.4.10, there is a column of $\Upsilon$ that contains at least $t 1^{\prime} s$, or $\Upsilon$ has a submatrix, $\Upsilon^{\prime}$ isomorphic to $I_{t}$. Suppose $\Upsilon$ has a column containing at least $t$ 1 's and let this column be labelled by $a$. Then there is a large subset of petals of $F$, which after relabelling we can consider to be $P_{2}, \ldots, P_{t}$, with the property that $x_{i} \in \operatorname{cl}\left\{P_{i} \cup a\right\}$ for $i \in\{2, \ldots, t\}$. Removing all petals of of $F$ that are not in $\left\{P_{1}, \ldots, P_{t}\right\}$ and reducing the elements of $\left\{P_{2}, \ldots, P_{t}\right\}$ to triads as in Lemma 5.3.3. gives the minor of $M$ described in $i$ ).

In the case where there is a submatrix, $\Upsilon^{\prime}$, of $\Upsilon$ that is isomorphic to $I_{t}$, relabel elements so that the petals that label the rows of the identity matrix are $P_{2}, \ldots, P_{t}$. Remove all petals not in $\left\{P_{1}, \ldots, P_{t}\right\}$ and remove all elements of $P_{1}$ that are not labels of columns of $\Upsilon^{\prime}$, in such a way as to keep connectivity. This gives a flower in which $P_{1}, P_{i}$ is blocked by single element $x_{i}$ and $x_{i} \in \operatorname{cl}\left(p_{i} \cup P_{i}\right)$ for some $p_{i} \in P_{1}$ with $p_{i} \neq p_{j}$ when $i \neq j$.

Lemma 5.3.6. Suppose $M$ has reduced standard representation

$$
\left(\begin{array}{cccccccccccc}
1 & 0 & 0 & 0 & 0 & \ldots & 0 & 0 & 1 & 1 & \ldots & 1 \\
0 & 1 & 1 & 0 & 0 & \ldots & 0 & 0 & 1 & 0 & \ldots & 0 \\
0 & 0 & 0 & 1 & 1 & \ldots & 0 & 0 & 0 & 1 & \ldots & 0 \\
\vdots & \vdots & \vdots & \vdots & \vdots & \ddots & \vdots & \vdots & \vdots & \vdots & \ddots & \vdots \\
0 & 0 & 0 & 0 & 0 & \ldots & 1 & 1 & 0 & 0 & \ldots & 1 \\
? & 0 & 1 & 0 & 1 & \ldots & 1 & 0 & ? & ? & \ldots & ? \\
? & 1 & 0 & 1 & 0 & \ldots & 0 & 1 & ? & ? & \ldots & ?
\end{array}\right)
$$

Then there is a rank- $(n+1)$ minor of this matroid that can be represented by the matrix from case (b) of Lemma 5.2.1 that is by the following matrix:

$$
\left(\begin{array}{cccccccccccccc}
1 & 1 & 0 & 0 & 0 & 0 & \ldots & 0 & 0 & 0 & 1 & 1 & \ldots & 1 \\
0 & 0 & 1 & 1 & 0 & 0 & \ldots & 0 & 0 & 0 & 1 & 0 & \ldots & 0 \\
0 & 0 & 0 & 0 & 1 & 1 & \ldots & 0 & 0 & 0 & 0 & 1 & \ldots & 0 \\
\vdots & \vdots & \vdots & \vdots & \vdots & \vdots & \vdots & \vdots & \vdots & \vdots & \vdots & \vdots & \vdots & \vdots \\
0 & 0 & 0 & 0 & 0 & 0 & \ldots & 1 & 1 & 0 & 0 & 0 & \ldots & 1 \\
1 & 0 & 1 & 0 & 1 & 0 & \ldots & 1 & 0 & 1 & ? & ? & \ldots & ? \\
0 & 1 & 0 & 1 & 0 & 1 & \ldots & 0 & 1 & 1 & ? & ? & \ldots & ?
\end{array}\right)
$$

Proof. There is a single element, $a$, in $P_{1}$. For some $j \in\{1, \ldots, n-1\}$, contract 
$x_{j}$. Now $a \in \operatorname{cl}\left(P_{j}\right)$ Therefore as every $x_{k} \in \operatorname{cl}\left(a \cup P_{k}\right)$, for $k \in\{2, \ldots, n\}$ it follows that $x_{k} \in \mathrm{cl}_{M / x_{j} \backslash a}\left(P_{j} \cup P_{k}\right)$. This means that $M / x_{j} \backslash a$ is a minor of $M$ isomorphic to one of the matroids in case $\left(b^{\prime}\right)$ Lemma 5.2.1

Lemma 5.3.7. Suppose that $M$ is represented by the following matrix

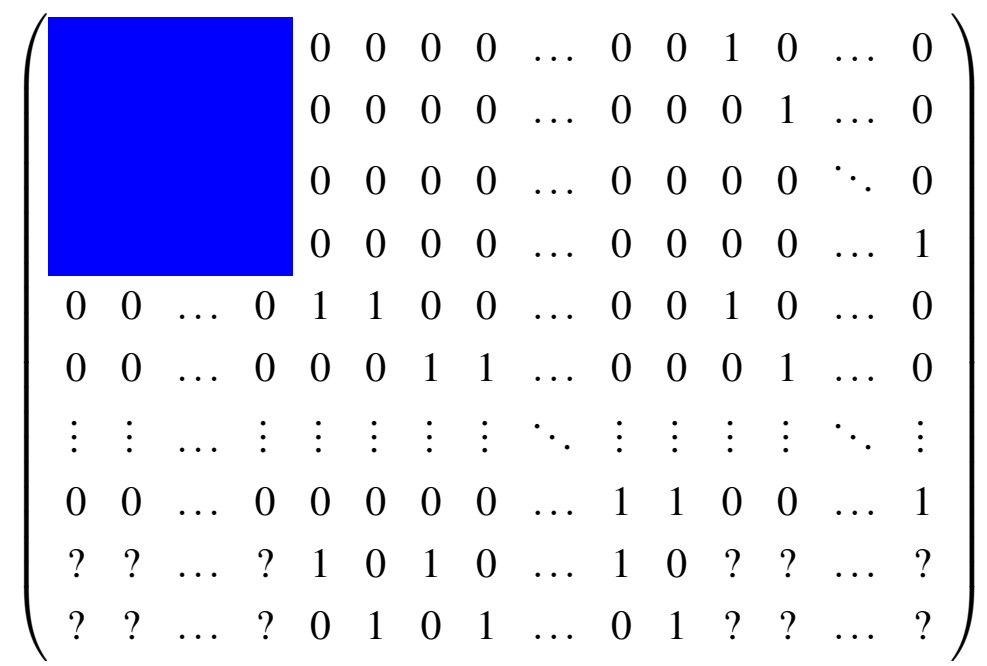

where the blue matrix represents $P_{1}$ and has rank $n-1$. If $n-1 \geq \sqrt{2.4 .1}(4 t+1)$, and for every $x \in X, x \in \operatorname{cl}\left(p_{i} \cup P_{i}\right)$ for some $p_{i} \in P_{1}$ with $p_{i} \neq p_{j}$ for all $p_{i}, p_{j} \in P_{1}$ and $P_{i} \neq P_{1}^{\prime}$. Then $M$ has a minor that can be represented by one of the following rank- $(t+2)$ matrices:

$$
\left(\begin{array}{ccccccccccc}
1 & 1 & 0 & 0 & 0 & 0 & \ldots & 0 & 0 & 0 & 1 \\
0 & 0 & 1 & 1 & 0 & 0 & \ldots & 0 & 0 & 0 & 1 \\
0 & 0 & 0 & 0 & 1 & 1 & \ldots & 0 & 0 & 0 & 1 \\
\vdots & \vdots & \vdots & \vdots & \vdots & \vdots & \vdots & \vdots & \vdots & \vdots & \vdots \\
0 & 0 & 0 & 0 & 0 & 0 & \ldots & 1 & 1 & 0 & 1 \\
1 & 0 & 1 & 0 & 1 & 0 & \ldots & 1 & 0 & 1 & ? \\
0 & 1 & 0 & 1 & 0 & 1 & \ldots & 0 & 1 & 1 & ?
\end{array}\right)
$$




$$
\left(\begin{array}{cccccccccccccc}
1 & 1 & 0 & 0 & 0 & 0 & \ldots & 0 & 0 & 0 & 1 & 1 & \ldots & 1 \\
0 & 0 & 1 & 1 & 0 & 0 & \ldots & 0 & 0 & 0 & 1 & 0 & \ldots & 0 \\
0 & 0 & 0 & 0 & 1 & 1 & \ldots & 0 & 0 & 0 & 0 & 1 & \ldots & 0 \\
\vdots & \vdots & \vdots & \vdots & \vdots & \vdots & \vdots & \vdots & \vdots & \vdots & \vdots & \vdots & \vdots & \vdots \\
0 & 0 & 0 & 0 & 0 & 0 & \ldots & 1 & 1 & 0 & 0 & 0 & \ldots & 1 \\
1 & 0 & 1 & 0 & 1 & 0 & \ldots & 1 & 0 & 1 & ? & ? & \ldots & ? \\
0 & 1 & 0 & 1 & 0 & 1 & \ldots & 0 & 1 & 1 & ? & ? & \ldots & ?
\end{array}\right)
$$

that is, $M$ can be represented by a matrix of the form given in case (a) or case (b) of Lemma 5.2.1.

Proof. We know $M \mid\left(P_{1} \cup\left\{g_{1}, g_{2}\right\}\right)$ is connected.

Claim 5.3.8. $M \mid\left(P_{1} \cup\left\{g_{1}, g_{2}\right\}\right) /\left\{g_{1}, g_{2}\right\}$ is connected.

Proof. First observe that $M \mid\left(P_{1} \cup\left\{g_{1}, g_{2}\right\}\right) / g_{1}$ is connected. For suppose not. Then there would be a 2-separation in $M \mid\left(P_{1} \cup\left\{g_{1}, g_{2}\right\}\right)$ with $g_{1}$ in the guts. This would be a 2-separation of $M$ not fully contained in a petal, which contradicts 3 of the definition of flower. Now if $M \mid\left(P_{1} \cup\left\{g_{1}, g_{2}\right\}\right)$ is not connected, then there is a 3-separation in $M \mid\left(P_{1} \cup\left\{g_{1}, g_{2}\right\}\right)$ with $g_{1}, g_{2}$ in the guts. This contradicts the maximality of $F$ established in the hypotheses of this chapter.

By Lemmas 5.3.5 we can assume that every $a_{i} \in P_{1}$ is in $\operatorname{cl}\left(x_{i} \cup P_{i}\right)$ and not in $\operatorname{cl}\left(x_{j} \cup P_{j}\right)$ for any $j \neq i$. Since $P_{1} \cup\left\{g_{1}, g_{2}\right\} /\left\{g_{1}, g_{2}\right\}$ is a connected matroid and

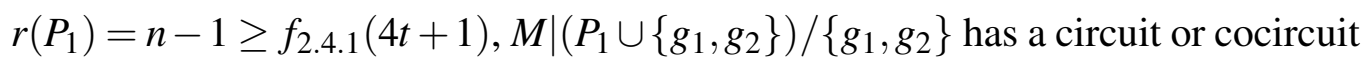
of size at least $4 t$

Suppose $M \mid\left(P_{1} \cup\left\{g_{1}, g_{2}\right\}\right) /\left\{g_{1}, g_{2}\right\}$ has a cocircuit of size $4 t$ as a minor. This means that $M \mid\left(P_{1} \cup\left\{g_{1}, g_{2}\right\}\right) /\left\{g_{1}, g_{2}\right\}$ has a parallel class of at least $4 t$ non-loop elements as a minor $N$. Coextending $N$ by $g_{1}$ and $g_{2}$ gives a minor of $M \mid\left(P_{1} \cup\right.$ $\left.\left\{g_{1}, g_{2}\right\}\right) /\left\{g_{1}, g_{2}\right\}$ with a parallel class, of at least $t$ elements and this parallel class is not in $\operatorname{cl}\left(\left\{g_{1}, g_{2}\right\}\right)$. The result then follows easily from Lemma 5.3.6.

Now suppose that $M \mid\left(P_{1} \cup\left\{g_{1}, g_{2}\right\} /\left\{g_{1}, g_{2}\right\}\right.$ contains a $t$-element circuit. Then there is a minor of $M \mid\left(P_{1} \cup\left\{g_{1}, g_{2}\right\}\right)$ that is a circuit $C$ and is such that $\mid C$ $\left\{g_{1}, g_{2}\right\} \mid=t$. The circuit $C$ may or may not contain one or both of $g_{1}$ and $g_{2}$. Let the basis elements of the circuit be $e_{1}, \ldots, e_{t-1}$ and the other element of the circuit be $a_{1}$. Therefore we now have a matroid that can be represented by the following 
matrix:

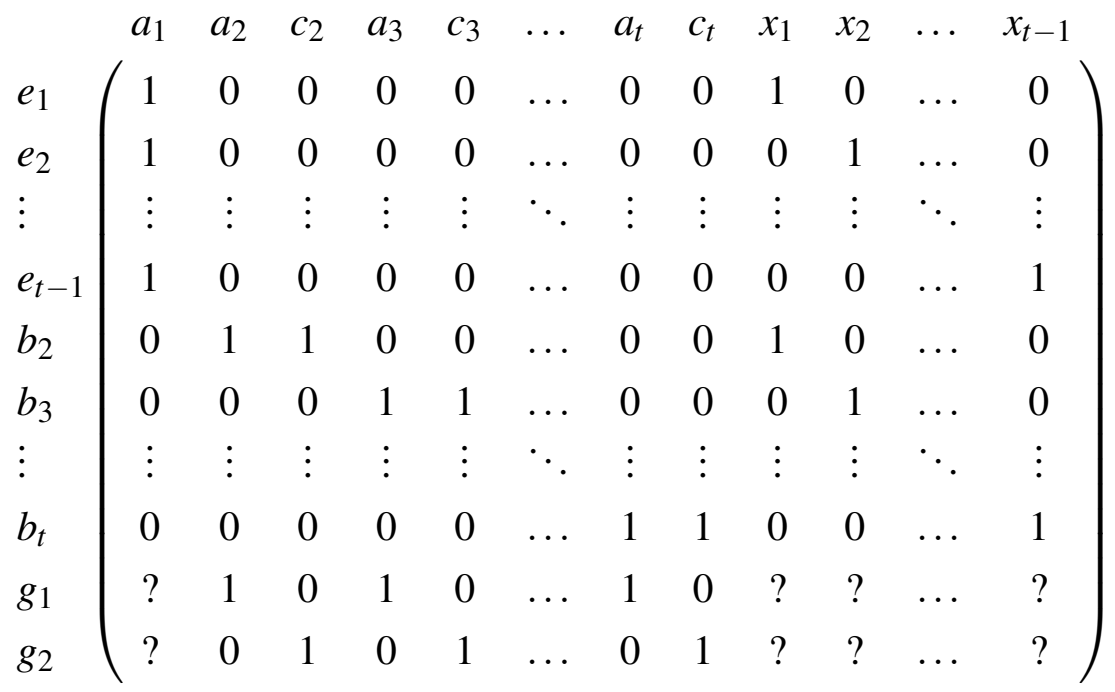

For all $i \in\{1, \ldots, t-1\}$ pivot on $M_{e_{i}, x_{i}}$ to get:

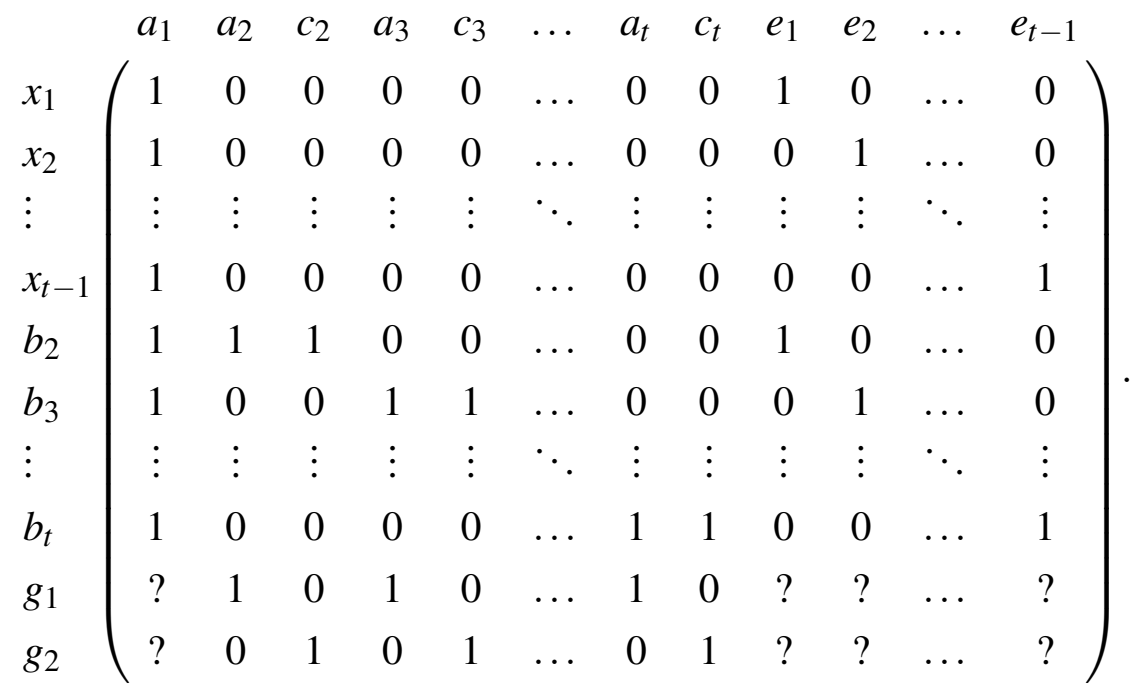

Deleting $e_{1}, \ldots, e_{t-1}$ and contracting $x_{1}, \ldots, x_{t-1}$, gives a rank- $(t+2)$ matrix of the following form. 


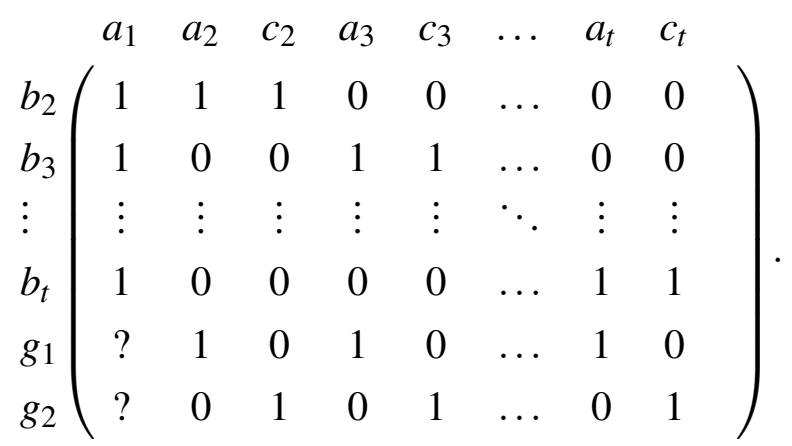

which is a rank $t+2$ matrix of the form given in case $(a)$ of Lemma 5.2.1.

Theorem 5.3.9. There is a function $\int_{5.3 .9}$ such that the following holds. Suppose $M$ is a matroid such that $M \backslash X$ has a paddle, $F$, with at least $n$ petals and that $F$ is blocked by $X$. If $n \geq f[5.3 .9(t)$ and $M$ can be represented by the reduced standard representation matrix,

$$
\left(\begin{array}{ccccccccc}
P_{1}^{\prime} & 0 & 0 & \ldots & 0 & Q_{1} & Q_{2} & \ldots & Q_{n} \\
0 & P_{2}^{\prime} & 0 & \ldots & 0 & Q_{1}^{\prime} & 0 & \ldots & 0 \\
0 & 0 & P_{3}^{\prime} & \ldots & 0 & 0 & Q_{2}^{\prime} & \ldots & 0 \\
\vdots & \vdots & \vdots & \ddots & \vdots & \vdots & \vdots & \ddots & \vdots \\
0 & 0 & 0 & \ldots & P_{t}^{\prime} & 0 & 0 & \ldots & Q_{n}^{\prime} \\
G_{1} & G_{2} & G_{3} & \ldots & G_{t} & ? & ? & \ldots & ?
\end{array}\right)
$$

then, $M$ has a $N\left(K_{3, t}\right)$-minor or an $M\left(K_{4, t}\right)$-minor.

Proof. Suppose $n \geq \sqrt{2.4 .10}\left(\max \left\{\int \frac{2.4 .1}{[2 .}\left(\max \left\{\int_{[5.2 .8}(t), \sqrt{[5.2 .4}(t)\right\}+2\right), \sqrt{5.2 .4}(t)+\right.\right.$ 1)).

Then by Lemma 5.3.5 $M$ has a minor $M^{\prime}$ that can be represented by one of the following matrices where 
$m \geq \max \left\{\int_{22.4 .1}\left(\max \left\{\int_{5.2 .8}(t), \int_{5.2 .4}(t)\right\}\right)+2, \sqrt{5.2 .4}(t)+1\right)$.

$$
\left(\begin{array}{cccccccccccc}
1 & 0 & 0 & 0 & 0 & \ldots & 0 & 0 & 1 & 1 & \ldots & 1 \\
0 & 1 & 1 & 0 & 0 & \ldots & 0 & 0 & 1 & 0 & \ldots & 0 \\
0 & 0 & 0 & 1 & 1 & \ldots & 0 & 0 & 0 & 1 & \ldots & 0 \\
\vdots & \vdots & \vdots & \vdots & \vdots & \ddots & \vdots & \vdots & \vdots & \vdots & \ddots & \vdots \\
0 & 0 & 0 & 0 & 0 & \ldots & 1 & 1 & 0 & 0 & \ldots & 1 \\
1 & 0 & 1 & 0 & 1 & \ldots & 1 & 0 & ? & ? & \ldots & ? \\
0 & 1 & 0 & 1 & 0 & \ldots & 0 & 1 & ? & ? & \ldots & ?
\end{array}\right) .
$$

where this matrix has at least rank $m+3$, or

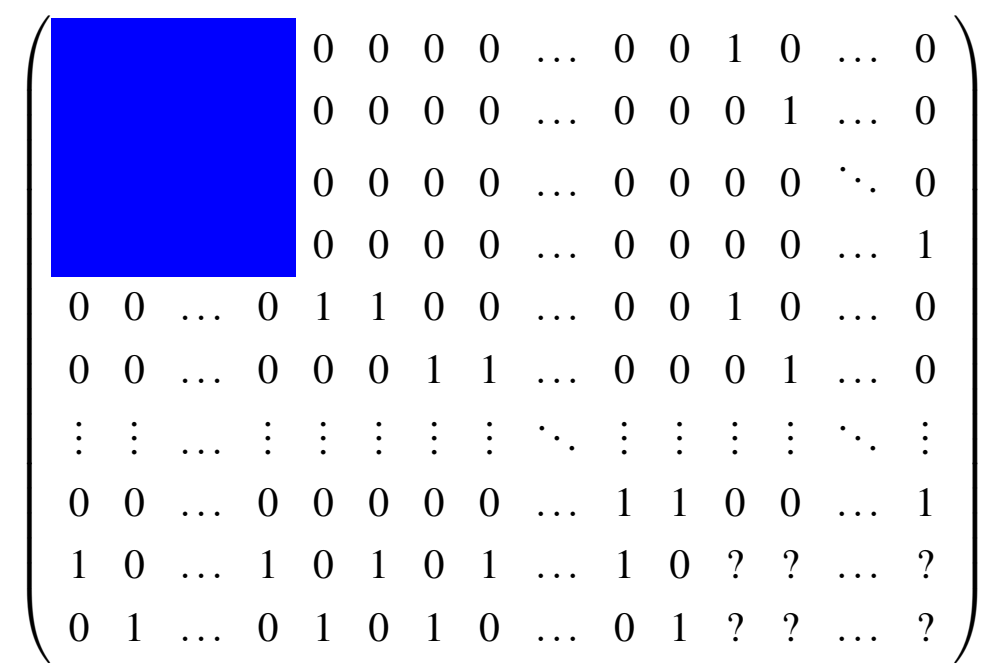

where the blue matrix represents the elements of $P_{1}$ and has rank $m-1$.

Consider the case where $M^{\prime}$ can be represented by

$$
\left(\begin{array}{cccccccccccc}
1 & 0 & 0 & 0 & 0 & \ldots & 0 & 0 & 1 & 1 & \ldots & 1 \\
0 & 1 & 1 & 0 & 0 & \ldots & 0 & 0 & 1 & 0 & \ldots & 0 \\
0 & 0 & 0 & 1 & 1 & \ldots & 0 & 0 & 0 & 1 & \ldots & 0 \\
\vdots & \vdots & \vdots & \vdots & \vdots & \ddots & \vdots & \vdots & \vdots & \vdots & \ddots & \vdots \\
0 & 0 & 0 & 0 & 0 & \ldots & 1 & 1 & 0 & 0 & \ldots & 1 \\
1 & 0 & 1 & 0 & 1 & \ldots & 1 & 0 & ? & ? & \ldots & ? \\
0 & 1 & 0 & 1 & 0 & \ldots & 0 & 1 & ? & ? & \ldots & ?
\end{array}\right)
$$

This has rank at least $f_{5.2 .4}(t)$ so, by Lemma 5.3.6 and Theorem 5.2.5, $M^{\prime}$ has a $N\left(K_{3, t}\right)$-minor. 
Now consider the case where $M^{\prime}$ is represented by

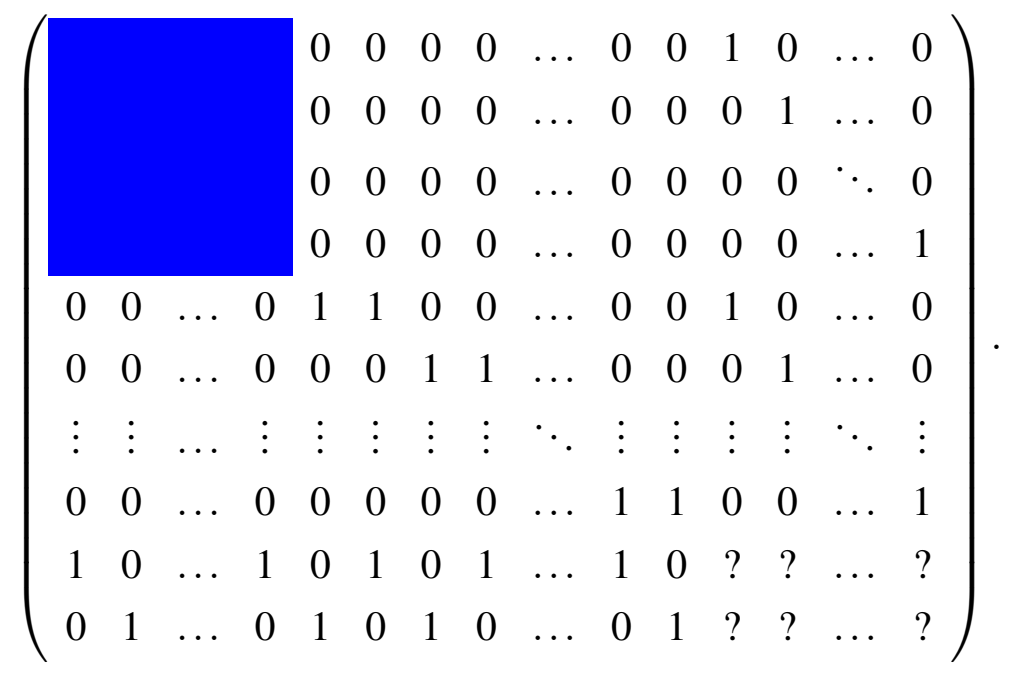

Since the blue matrix has at least rank $\int_{2.4 .1}\left(\max \left\{\int_{5.2 .8}(t), f_{5.2 .4}(t)\right\}+2\right)$, by Lemma 5.3.7 $M^{\prime}$ has a minor $M^{\prime \prime}$ of rank $m^{\prime} \geq \max \left\{f_{5.2 .8}(t), f_{5.2 .4}(t)\right\}+2$ of one of the following forms:

$$
\left(\begin{array}{ccccccccccc}
1 & 1 & 0 & 0 & 0 & 0 & \ldots & 0 & 0 & 0 & 1 \\
0 & 0 & 1 & 1 & 0 & 0 & \ldots & 0 & 0 & 0 & 1 \\
0 & 0 & 0 & 0 & 1 & 1 & \ldots & 0 & 0 & 0 & 1 \\
\vdots & \vdots & \vdots & \vdots & \vdots & \vdots & \vdots & \vdots & \vdots & \vdots & \vdots \\
0 & 0 & 0 & 0 & 0 & 0 & \ldots & 1 & 1 & 0 & 1 \\
1 & 0 & 1 & 0 & 1 & 0 & \ldots & 1 & 0 & 1 & ? \\
0 & 1 & 0 & 1 & 0 & 1 & \ldots & 0 & 1 & 1 & ?
\end{array}\right)
$$

or

$$
\left(\begin{array}{cccccccccccccc}
1 & 1 & 0 & 0 & 0 & 0 & \ldots & 0 & 0 & 0 & 1 & 1 & \ldots & 1 \\
0 & 0 & 1 & 1 & 0 & 0 & \ldots & 0 & 0 & 0 & 1 & 0 & \ldots & 0 \\
0 & 0 & 0 & 0 & 1 & 1 & \ldots & 0 & 0 & 0 & 0 & 1 & \ldots & 0 \\
\vdots & \vdots & \vdots & \vdots & \vdots & \vdots & \vdots & \vdots & \vdots & \vdots & \vdots & \vdots & \vdots & \vdots \\
0 & 0 & 0 & 0 & 0 & 0 & \ldots & 1 & 1 & 0 & 0 & 0 & \ldots & 1 \\
1 & 0 & 1 & 0 & 1 & 0 & \ldots & 1 & 0 & 1 & ? & ? & \ldots & ? \\
0 & 1 & 0 & 1 & 0 & 1 & \ldots & 0 & 1 & 1 & ? & ? & \ldots & ?
\end{array}\right)
$$

By Lemma 5.2.5 and Lemma 5.2.9 this means that $M^{\prime \prime}$ and hence $M$ has a $N\left(K_{3, t}\right)$ or $M\left(K_{4, t}\right)$-minor. 


\subsubsection{Case $(c)$}

Consider the matrix $(c)$ from Lemma 5.1.2, that is the matrix given below:

$$
\left(\begin{array}{cccccccccc}
P_{1} & 0 & 0 & \ldots & 0 & 0 & Q_{1} & 0 & \ldots & 0 \\
0 & P_{2} & 0 & \ldots & 0 & 0 & Q_{1}^{\prime} & Q_{2} & \ldots & 0 \\
0 & 0 & P_{3} & \ldots & 0 & 0 & 0 & Q_{2}^{\prime} & \ldots & 0 \\
\vdots & \vdots & \vdots & \ddots & \vdots & \vdots & \vdots & \vdots & \ddots & \vdots \\
0 & 0 & 0 & \ldots & P_{n-1} & 0 & 0 & 0 & \ldots & Q_{n-1} \\
0 & 0 & 0 & \ldots & 0 & P_{n} & 0 & 0 & \ldots & Q_{n-1}^{\prime} \\
G_{1} & G_{2} & G_{3} & \ldots & G_{n-1} & G_{n} & ? & ? & \ldots & ?
\end{array}\right)
$$

where, for $i \in\{1, \ldots, n\}, P_{i}^{\prime}, Q_{i}$ and $G_{i}$ are matrices where

1. $G_{i}$ has two rows and the rows of $G_{i}$ are labelled by a maximal independent set contained in the guts petal of $F$,

2. $G_{1}, \ldots, G_{n}$ represent matrices that each contain at least one non-zero entry in every row,

3. the rows of $P_{i}^{\prime}$ are labelled by the basis elements of $\left(M \mid P_{i}\right)$,

4. the columns containing columns of $P_{i}^{\prime}$ label the elements of $P_{i}-B$, and

5. For $i \in\{1, \ldots, n-1\}, Q_{i}$ represents $\left(r\left(P_{i}\right)-2\right) \times 1$ matrices with rows labelled by the basis elements of $P_{i}$ and for every $i \in\{1, \ldots, n-1\}$ there is a 1 in some row of $Q_{i}$.

6. For $i \in\{1, \ldots, n-1\}, Q_{i}^{\prime}$ represents $\left(r\left(P_{i+1}\right)-2\right) \times 1$ matrices with rows labelled by the basis elements of $P_{i+1}$ and for every $i \in\{1, \ldots, n-1\}$ there is a 1 in some row of $Q_{i}$.

Throughout this case we assume that $M$ is represented by the matrix above, and $F=\left(P_{1}, \ldots, P_{n}, G\right)$, where the elements of $P_{i}$ are the elements labelling rows and columns of $P_{i}^{\prime}$, and $G$ is the elements labelling rows of $G_{1}$.

In this section we proceed as follows. We find a minor of $M$ represented by the 
following matrix:

$$
\left(\begin{array}{ccccccccccccccccc}
1 & 1 & 0 & 0 & 0 & 0 & 0 & 0 & \ldots & 0 & 0 & 0 & 1 & 0 & 0 & \ldots & 0 \\
0 & 0 & 1 & 1 & 0 & 0 & 0 & 0 & \ldots & 0 & 0 & 0 & 1 & 1 & 0 & \ldots & 0 \\
0 & 0 & 0 & 0 & 1 & 1 & 0 & 0 & \ldots & 0 & 0 & 0 & 0 & 1 & 1 & \ldots & 0 \\
0 & 0 & 0 & 0 & 0 & 0 & 1 & 1 & \ldots & 0 & 0 & 0 & 0 & 0 & 1 & \ldots & 0 \\
\vdots & \vdots & \vdots & \vdots & \vdots & \vdots & : \vdots & \vdots & \vdots & \vdots & \vdots & \vdots & \vdots & \vdots & \vdots & \vdots & \vdots \\
0 & 0 & 0 & 0 & 0 & 0 & 0 & 0 & \ldots & 1 & 1 & 0 & 0 & 0 & 0 & \ldots & 1 \\
1 & 0 & 1 & 0 & 1 & 0 & 1 & 0 & \ldots & 1 & 0 & 1 & ? & ? & ? & \ldots & ? \\
0 & 1 & 0 & 1 & 0 & 1 & 0 & 1 & \ldots & 0 & 1 & 1 & ? & ? & ? & \ldots & ?
\end{array}\right)
$$

After this, this case as been reduced to the previously solved problem of finding the unavoidable minors of the above matrix.

Since $M \backslash X$ has an $M\left(K_{3, n}\right)$-minor the following result is obvious.

Lemma 5.3.10. Every submatrix of the representation of $M$ given in (c) of Lemma 5.1.2 with rows labelled by elements of $P_{i}$, for $i \in\{2, \ldots, n-1\}$, must contain a submatrix of one of the following forms:

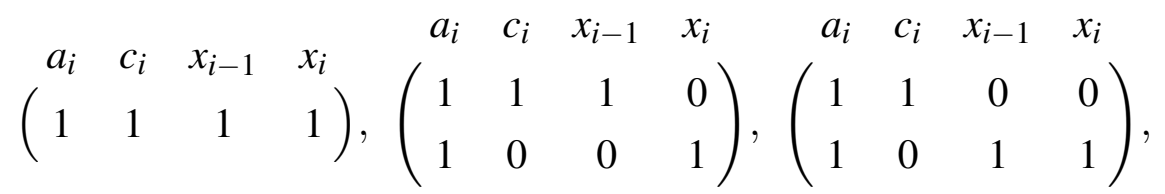

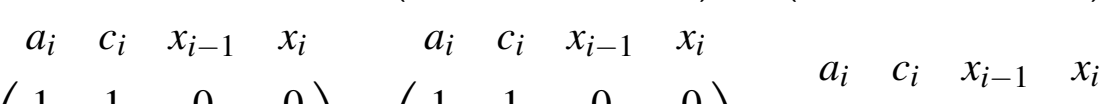

$$
\begin{aligned}
& \left(\begin{array}{llll}
1 & 1 & 0 & 0 \\
1 & 0 & 1 & 0 \\
1 & 0 & 0 & 1
\end{array}\right),\left(\begin{array}{llll}
1 & 1 & 0 & 0 \\
1 & 0 & 1 & 0 \\
0 & 1 & 0 & 1
\end{array}\right),\left(\begin{array}{cccc}
a_{i} & c_{i} & x_{i-1} & x_{i} \\
1 & 1 & 1 & 0 \\
1 & 1 & 0 & 1
\end{array}\right) .
\end{aligned}
$$

For the next few pages, and where it arises throughout the thesis we may use ? to denote unknown row or column labels.

Lemma 5.3.11. Suppose $P_{i}$ is a petal of $F$ and $C, D \subseteq P_{i}$ with the following properties.

1. there is some $M^{\prime}=M \backslash D / C$ for $C, D \subseteq P_{i}$ such that $M^{\prime} \backslash X$ has a flower $F^{\prime}=\left(P_{1}, \ldots P_{i-1}, P_{i}^{\prime}, P_{i+1}, \ldots, P_{n}\right)$

2. every displayed 3-separation of $M^{\prime} \backslash X$ is blocked by some $x \in X$ and, 
3. $M \mid P_{i}^{\prime}$ can be represented by one of the following matrices:

$$
\begin{aligned}
& \begin{array}{llllllll}
a_{i} & c_{i} & x_{i-1} & x_{i} & a_{i} & c_{i} & x_{i-1} & x_{i}
\end{array} \\
& \left(\begin{array}{llll}
1 & 1 & 1 & 0 \\
1 & 0 & 0 & 1
\end{array}\right),\left(\begin{array}{llll}
1 & 1 & 0 & 0 \\
1 & 0 & 1 & 1
\end{array}\right), \\
& \begin{array}{llllllll}
a_{i} & c_{i} & x_{i-1} & x_{i} & a_{i} & c_{i} & x_{i-1} & x_{i}
\end{array} \\
& \left(\begin{array}{llll}
1 & 1 & 0 & 0 \\
1 & 0 & 1 & 0 \\
1 & 0 & 0 & 1
\end{array}\right),\left(\begin{array}{llll}
1 & 1 & 0 & 0 \\
1 & 0 & 1 & 0 \\
0 & 1 & 0 & 1
\end{array}\right) .
\end{aligned}
$$

Then $M$ has a minor $M^{\prime \prime}=M^{\prime} \backslash D^{\prime} / C^{\prime}$ that is blocked by $X$, with the property that such that $M^{\prime \prime}$ has a swirl-like pseudo-flower $F^{\prime \prime}=\left(P_{1}, \ldots, P_{i-1}, P_{i}^{\prime \prime}, P_{i+1}, \ldots, P_{n}\right)$ and $M^{\prime \prime} \mid P_{i}^{\prime \prime}$ can be represented by

$$
\left(\begin{array}{cccc}
? & ? & x_{i-1} & x_{i} \\
1 & 1 & 1 & 1
\end{array}\right) .
$$

Proof. Let $M^{\prime} \mid P_{i}^{\prime}:=P$ and let the final rows of $P$ be labelled $p_{1}, \ldots, p_{i}$ for some $i \in\{1,2\}$. Suppose $P$ is represented by the following matrix:

$$
\begin{aligned}
& \begin{array}{llll}
a_{i} & c_{i} & x_{i-1} & x_{i}
\end{array} \\
& b_{1}\left(\begin{array}{llll}
1 & 1 & a & b \\
1 & 0 & c & d
\end{array}\right) \text {. }
\end{aligned}
$$

Pivoting on $M_{a_{i}, p_{1}}$ gives

$$
\begin{aligned}
& \begin{array}{llll}
p_{1} & c_{i} & x_{i-1} & x_{i}
\end{array} \\
& b_{i}\left(\begin{array}{cccc}
1 & 1 & a+c & b+d \\
1 & 0 & c & d
\end{array}\right) .
\end{aligned}
$$

In the cases above exactly one of $a$ and $c$ and exactly one of $b$ and $d$ is equal to 1 .

Contracting $a_{i}$ then gives $\left(\begin{array}{cccc}p_{1} & c_{i} & x_{i-1} & x_{i} \\ 1 & 1 & 1 & 1\end{array}\right)$. 
Suppose $P$ is represented by

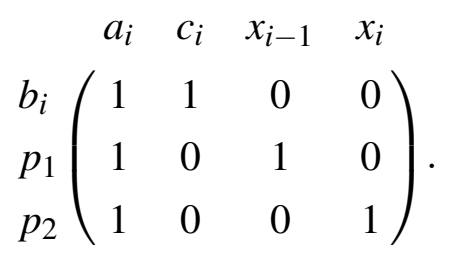

Pivot on $M_{a_{i}, p_{1}}$ to get

$$
\begin{aligned}
& \begin{array}{llll}
p_{1} & c_{i} & x_{i-1} & x_{i}
\end{array} \\
& \begin{array}{l}
b_{i} \\
a_{i} \\
p_{2}
\end{array}\left(\begin{array}{llll}
1 & 1 & 1 & 0 \\
1 & 0 & 1 & 0 \\
1 & 0 & 0 & 1
\end{array}\right)
\end{aligned}
$$

and on $M_{p_{1}, p_{2}}$ to get

$$
\begin{aligned}
& \begin{array}{llll}
p_{2} & c_{i} & x_{i-1} & x_{i}
\end{array} \\
& \begin{array}{c}
b_{i} \\
a_{i} \\
p_{1}
\end{array}\left(\begin{array}{llll}
1 & 1 & 1 & 1 \\
1 & 0 & 1 & 0 \\
1 & 0 & 0 & 1
\end{array}\right) .
\end{aligned}
$$

This gives a $\left(\begin{array}{cccc}p_{2} & c_{i} & x_{i-1} & x_{i} \\ 1 & 1 & 1 & 1\end{array}\right)$ minor.

Suppose $P$ is represented by

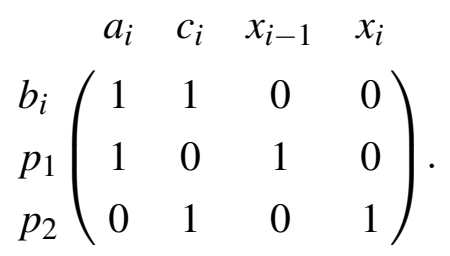

Pivot on $M_{a_{i}, p_{1}}$ and $M_{c_{i}, p_{2}}$ to get

$$
\begin{aligned}
& p_{1} \quad p_{2} \quad x_{i-1} \quad x_{i} \\
& \begin{array}{l}
b_{i} \\
c_{i} \\
a_{2}
\end{array}\left(\begin{array}{llll}
1 & 1 & 1 & 1 \\
1 & 0 & 1 & 0 \\
0 & 1 & 0 & 1
\end{array}\right)
\end{aligned}
$$

Contracting $a_{i}$ and $c_{i}$ gives $\left(\begin{array}{cccc}p_{2} & c_{i} & x_{i-1} & x_{i} \\ 1 & 1 & 1 & 1\end{array}\right)$. 
Unfortunately when we have a petal of the form $\left(\begin{array}{ccccc}a_{i} & c_{i} & x_{i-1} & x_{i} \\ 1 & 1 & 1 & 0 \\ 1 & 1 & 0 & 1\end{array}\right)$, we cannot find a minor of the form $\left(\begin{array}{cccc}p_{2} & c_{i} & x_{i-1} & x_{i} \\ 1 & 1 & 1 & 1\end{array}\right)$. To deal with this case we instead look at pairs of petals. If for some $i \in\{1, \ldots, n\}$ the matroid $M \mid P_{i}$ does not have $\begin{array}{llll}p_{2} & c_{i} & x_{i-1} & x_{i}\end{array}$

a minor of the form $\left(\begin{array}{llll}1 & 1 & 1 & 1\end{array}\right)$, then $M \mid P_{i}$ must have a minor of the following form:

$$
\begin{aligned}
& \begin{array}{llll}
? & ? & x_{i-1} & x_{i}
\end{array} \\
& b_{i}\left(\begin{array}{llll}
1 & 1 & 1 & 0 \\
1 & 1 & 0 & 1
\end{array}\right) .
\end{aligned}
$$

We look at what happens if we have two adjacent petals with minors of this form, or a petal of this form followed by a petal of the form $\left(\begin{array}{llll}1 & 1 & 1 & 1\end{array}\right)$.

Lemma 5.3.12. Let $N$ be a binary matroid with reduced standard representation of the form

$$
\left(\begin{array}{lllllll}
1 & 1 & 0 & 0 & 1 & 0 & 0 \\
1 & 1 & 0 & 0 & 0 & 1 & 0 \\
0 & 0 & 1 & 1 & 0 & 1 & 0 \\
0 & 0 & 1 & 1 & 0 & 0 & 1
\end{array}\right)
$$

or

$$
\left(\begin{array}{lllllll}
1 & 1 & 0 & 0 & 1 & 0 & 0 \\
1 & 1 & 0 & 0 & 0 & 1 & 0 \\
0 & 0 & 1 & 1 & 0 & 1 & 1
\end{array}\right)
$$

where the final three columns of the matrix are labelled by $y_{i}, y_{i+1}, y_{i+2}$. Then $N$ has a minor of form $\left[\begin{array}{llll}1 & 1 & 1 & 1\end{array}\right]$, where the final two columns are labelled by $y_{i}$ and $y_{i+2}$.

Proof. Suppose $\mathrm{N}$ is represented by the following matrix:

$$
\begin{aligned}
& \begin{array}{lllllll}
a_{i} & c_{i} & a_{i+1} & c_{i+1} & y_{i} & y_{i+1} & y_{i+2}
\end{array} \\
& \begin{array}{l}
b_{i} \\
p_{i} \\
b_{i+1} \\
p_{i+1}
\end{array}\left(\begin{array}{ccccccc}
1 & 1 & 0 & 0 & 1 & 0 & 0 \\
1 & 1 & 0 & 0 & 0 & 1 & 0 \\
0 & 0 & 1 & 1 & 0 & 1 & 0 \\
0 & 0 & 1 & 1 & 0 & 0 & 1
\end{array}\right) .
\end{aligned}
$$


Pivot on $M_{p_{i}, c_{i}}$ and $M_{p_{i+1}, c_{i+1}}$ to get:

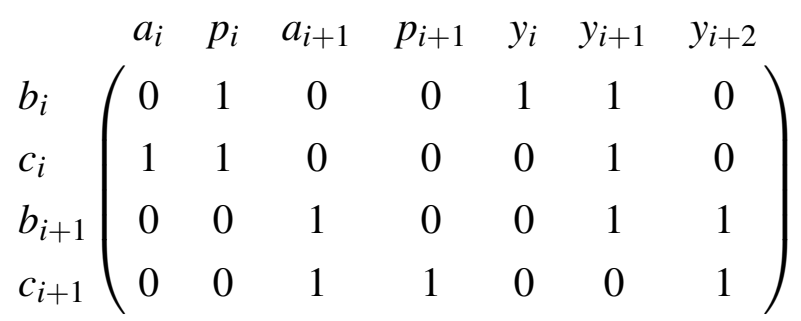

Finally pivot on $M_{b_{i+1}, y_{i+1}}$ to get

$$
\begin{aligned}
& \begin{array}{lllllll}
a_{i} & p_{i} & a_{i+1} & p_{i+1} & y_{i} & b_{i+1} & y_{i+2}
\end{array} \\
& \begin{array}{l}
b_{i} \\
c_{i} \\
y_{i+1} \\
c_{i+1}
\end{array}\left(\begin{array}{lllllll}
0 & 1 & 1 & 0 & 1 & 1 & 1 \\
1 & 1 & 1 & 0 & 0 & 1 & 1 \\
0 & 0 & 1 & 0 & 0 & 1 & 1 \\
0 & 0 & 1 & 1 & 0 & 0 & 1
\end{array}\right)
\end{aligned}
$$

Deleting all but the first row and the first and fourth columns of this give the matrix $\left(\begin{array}{llll}1 & 1 & 1 & 1\end{array}\right)$, where the final two columns are labelled by $y_{i}$ and $y_{i+2}$.

Now suppose $N$ is represented by the following matrix.

$$
\begin{aligned}
& \begin{array}{lllllll}
a_{i} & c_{i} & a_{i+1} & c_{i+1} & x_{i} & x_{i+1} & x_{i+2}
\end{array} \\
& \begin{array}{l}
b_{i} \\
p_{i} \\
b_{i+1}
\end{array}\left(\begin{array}{ccccccc}
1 & 1 & 0 & 0 & 1 & 0 & 0 \\
1 & 1 & 0 & 0 & 0 & 1 & 0 \\
0 & 0 & 1 & 1 & 0 & 1 & 1
\end{array}\right)
\end{aligned}
$$

Pivoting on $M_{b_{i}, a_{i}}$ give the following matrix

$$
\begin{aligned}
& \begin{array}{lllllll}
b_{i} & c_{i} & a_{i+1} & c_{i+1} & x_{i} & x_{i+1} & x_{i+2}
\end{array} \\
& \begin{array}{l}
a_{i} \\
p_{i} \\
b_{i+1}
\end{array}\left(\begin{array}{ccccccc}
1 & 1 & 0 & 0 & 1 & 0 & 0 \\
1 & 0 & 0 & 0 & 1 & 1 & 0 \\
0 & 0 & 1 & 1 & 0 & 1 & 1
\end{array}\right) .
\end{aligned}
$$


If we now pivot on $M_{p_{i}, x_{i+1}}$ we get

$$
\begin{aligned}
& b_{i} \quad c_{i} \quad a_{i+1} \quad c_{i+1} \quad x_{i} \quad p_{i} \quad x_{i+2} \\
& \begin{array}{l}
a_{i} \\
x_{i+1} \\
b_{i+1}
\end{array}\left(\begin{array}{ccccccc}
1 & 1 & 0 & 0 & 1 & 0 & 0 \\
1 & 0 & 1 & 1 & 1 & 1 & 1 \\
0 & 0 & 1 & 1 & 0 & 1 & 1
\end{array}\right) \text {. }
\end{aligned}
$$

Deleting the first and last rows and the second, third and sixth column gives the matrix $\left(\begin{array}{llll}1 & 1 & 1 & 1\end{array}\right)$, where the final two columns are labelled by $x_{i}$ and $x_{i+2}$.

Now all that remains is to consider the first and last petals. The first petal must contain a submatrix of one of the following forms

$$
\left(\begin{array}{ccc}
? & ? & x_{1} \\
1 & 1 & 1
\end{array}\right)
$$

or

$$
\begin{gathered}
? \\
\left.? \begin{array}{ccc}
1 & 1 & 0 \\
1 & 0 & 1
\end{array}\right) .
\end{gathered}
$$

It is easy to see that we can perform a change of basis and find a minor of $M^{\prime}$ with flower $F=\left(P_{1}^{\prime}, P_{2}, \ldots, P_{n}\right)$ such that $M \mid P_{1}$ can be represented by the following $? \quad x_{1}$

matrix $\left(\begin{array}{lll}1 & 1 & 1\end{array}\right)$. Clearly we can use the same argument to obtain a minor of $M \mid P_{n}$ represented by $\left(\begin{array}{ccc}? & ? & x_{n-1} \\ ? & ? & x_{1} \\ 1 & 1 & 1\end{array}\right)$. Since the columns labelled by the blocking elements have remained unchanged we get the following: 
Lemma 5.3.13. If $n \geq 2 t$ then $M$ has a rank- $(t+2)$ minor of the form:

$$
\left(\begin{array}{ccccccccccccccccc}
1 & 1 & 0 & 0 & 0 & 0 & 0 & 0 & \ldots & 0 & 0 & 0 & 1 & 0 & 0 & \ldots & 0 \\
0 & 0 & 1 & 1 & 0 & 0 & 0 & 0 & \ldots & 0 & 0 & 0 & 1 & 1 & 0 & \ldots & 0 \\
0 & 0 & 0 & 0 & 1 & 1 & 0 & 0 & \ldots & 0 & 0 & 0 & 0 & 1 & 1 & \ldots & 0 \\
0 & 0 & 0 & 0 & 0 & 0 & 1 & 1 & \ldots & 0 & 0 & 0 & 0 & 0 & 1 & \ldots & 0 \\
\vdots & \vdots & \vdots & \vdots & \vdots & \vdots & \vdots & \vdots & \vdots & \vdots & \vdots & \vdots & \vdots & \vdots & \vdots & \vdots & \vdots \\
0 & 0 & 0 & 0 & 0 & 0 & 0 & 0 & \ldots & 1 & 1 & 0 & 0 & 0 & 0 & \ldots & 1 \\
1 & 0 & 1 & 0 & 1 & 0 & 1 & 0 & \ldots & 1 & 0 & 1 & 0 & 0 & 0 & \ldots & 0 \\
0 & 1 & 0 & 1 & 0 & 1 & 0 & 1 & \ldots & 0 & 1 & 1 & 0 & 0 & 0 & \ldots & 0
\end{array}\right)
$$

Theorem 5.3.14. There is a function $\sqrt{5.3 .14}$ such that the following holds. If $n \geq$ 55.3.14 $(t)$, and $M$ is represented by the matrix given in case $c^{\prime}$ of Lemma 5.1.2. then $M$ has a rank-t double wheel as a minor.

Proof. Let $n \geq 2 \sqrt{5.2 .13}(t)$. Then, by Lemma 5.3.13, $M$ has a minor $M^{\prime}$ of rank at least $5.5 .2 .13(t)$ that can be represented by the following matrix:

$$
\left(\begin{array}{ccccccccccccccccc}
1 & 1 & 0 & 0 & 0 & 0 & 0 & 0 & \ldots & 0 & 0 & 0 & 1 & 0 & 0 & \ldots & 0 \\
0 & 0 & 1 & 1 & 0 & 0 & 0 & 0 & \ldots & 0 & 0 & 0 & 1 & 1 & 0 & \ldots & 0 \\
0 & 0 & 0 & 0 & 1 & 1 & 0 & 0 & \ldots & 0 & 0 & 0 & 0 & 1 & 1 & \ldots & 0 \\
0 & 0 & 0 & 0 & 0 & 0 & 1 & 1 & \ldots & 0 & 0 & 0 & 0 & 0 & 1 & \ldots & 0 \\
\vdots & \vdots & \vdots & \vdots & \vdots & \vdots & \vdots & \vdots & \vdots & \vdots & \vdots & \vdots & \vdots & \vdots & \vdots & \vdots & \vdots \\
0 & 0 & 0 & 0 & 0 & 0 & 0 & 0 & \ldots & 1 & 1 & 0 & 0 & 0 & 0 & \ldots & 1 \\
1 & 0 & 1 & 0 & 1 & 0 & 1 & 0 & \ldots & 1 & 0 & 1 & 0 & 0 & 0 & \ldots & 0 \\
0 & 1 & 0 & 1 & 0 & 1 & 0 & 1 & \ldots & 0 & 1 & 1 & 0 & 0 & 0 & \ldots & 0
\end{array}\right)
$$

By Theorem 5.2.13 $M^{\prime}$ and hence $M$ has a rank- $t$ double wheel as a minor.

\subsection{Proof of Theorem 5.0.1}

Putting all this together we get a proof of Theorem 5.0.1.

Theorem 5.0.1 There is a function 55.0 .1 such that the following holds. Suppose $M$ is a binary matroid such that for some coindependent set $X$, the matroid $M \backslash X$ has a paddle partition $F=\left(P_{1}, \ldots, P_{n}\right)$. Further suppose that every 3-separation 
of $M \backslash X$ displayed by $F$ is blocked by some $x \in X$. If $n \geq \int_{5.0 .1}(t)$, then $M$ has a minor isomorphic to one of the following:
i) $N\left(K_{3, t}\right)$,
ii) $M\left(K_{4, t}\right)$,

iii) a rank-t double wheel.

Proof. Let $n \geq \sqrt{5.1 .2}\left(\max \left\{f_{5.2 .3}(t), f_{5.2 .2}(t), f_{5.2 .4}(t)\right\}\right)$. By Lemma 5.1.2, there is a minor of $M$ that has a reduced standard representation matrix of one of the following forms:

$$
\left(\begin{array}{cccccc}
P_{1}^{\prime} & 0 & 0 & \ldots & 0 & Q_{1} \\
0 & P_{2}^{\prime} & 0 & \ldots & 0 & Q_{2} \\
0 & 0 & P_{3}^{\prime} & \ldots & 0 & Q_{3} \\
\vdots & \vdots & \vdots & \ddots & \vdots & \vdots \\
0 & 0 & 0 & \ldots & P_{t}^{\prime} & Q_{n} \\
G_{1} & G_{2} & G_{2} & \ldots & G_{t} & ?
\end{array}\right)
$$

(a)

$$
\left(\begin{array}{ccccccccc}
P_{1}^{\prime} & 0 & 0 & \ldots & 0 & Q_{1} & Q_{2} & \ldots & Q t-13 \\
0 & P_{2}^{\prime} & 0 & \ldots & 0 & Q_{1}^{\prime} & 0 & \ldots & 0 \\
0 & 0 & P_{3}^{\prime} & \ldots & 0 & 0 & Q_{2}^{\prime} & \ldots & 0 \\
\vdots & \vdots & \vdots & \ddots & \vdots & \vdots & \vdots & \ddots & \vdots \\
0 & 0 & 0 & \ldots & P_{t}^{\prime} & 0 & 0 & \ldots & Q_{t-1}^{\prime} \\
G_{1} & G_{2} & G_{3} & \ldots & G_{t} & ? & ? & \ldots & ?
\end{array}\right)
$$

(b)

$$
\left(\begin{array}{cccccccccc}
P_{1}^{\prime} & 0 & 0 & \ldots & 0 & 0 & Q_{1} & 0 & \ldots & 0 \\
0 & P_{2}^{\prime} & 0 & \ldots & 0 & 0 & Q_{1}^{\prime} & Q_{2} & \ldots & 0 \\
0 & 0 & P_{3}^{\prime} & \ldots & 0 & 0 & 0 & Q_{2}^{\prime} & \ldots & 0 \\
\vdots & \vdots & \vdots & \ddots & \vdots & \vdots & \vdots & \vdots & \ddots & \vdots \\
0 & 0 & 0 & \ldots & P_{n-1}^{\prime} & 0 & 0 & 0 & \ldots & Q_{n-1} \\
0 & 0 & 0 & \ldots & 0 & P_{n}^{\prime} & 0 & 0 & \ldots & Q_{n-1}^{\prime} \\
G_{1} & G_{2} & G_{3} & \ldots & G_{t-1} & G_{t} & ? & ? & \ldots & ?
\end{array}\right)
$$


(c)

Where, for $i \in\{1, \ldots, n\}, P_{i}^{\prime}, Q_{i}$ and $G_{i}$ are matrices such that

1. $G_{i}$ has two rows and the rows of $G_{i}$ are labelled by a maximal independent set contained in the guts petal of $F$,

2. $G_{1}, \ldots, G_{n}$ represent matrices that each contain at least one non-zero entry in every row,

3. the rows of $P_{i}^{\prime}$ are labelled by the basis elements of $\left(M \mid P_{i}\right)$,

4. the columns containing columns of $P_{i}^{\prime}$ label the elements of $P_{i}-B$, and

5. For $i \in\{1, \ldots, n-1\}, Q_{i}$ represents $\left(r\left(P_{i}\right)-2\right) \times 1$ matrices with rows labelled by the basis elements of $P_{i}$ and for every $i \in\{1, \ldots, n-1\}$ there is a 1 in some row of $Q_{i}$.

6. For $i \in\{1, \ldots, n-1\}, Q_{i}^{\prime}$ represents $\left(r\left(P_{i+1}\right)-2\right) \times 1$ matrices with rows labelled by the basis elements of $P_{i+1}$ and for every $i \in\{1, \ldots, n-1\}$ there is a 1 in some row of $Q_{i}$.

If $M$ has a minor $M^{\prime}$ of form $(a)$ then, since $r\left(M^{\prime}\right) \geq \sqrt{5.2 .4}(t)+2$, by Lemma 5.3.9 $M^{\prime}$ and hence $M$ has a $N\left(K_{3, t}\right)$-minor. If $M$ has a minor $M^{\prime}$ of form $(b)$ then, since $r\left(M^{\prime}\right) \geq \sqrt{5.3 .9}(t)+2$, by Lemma 5.3.9 $M^{\prime}$ and hence $M$ has a $N\left(K_{3, t}\right)$-minor or an $M\left(K_{4, t}\right)$-minor. If $M$ has a minor $M^{\prime}$ of form $(c)$ then, since $r\left(M^{\prime}\right) \geq \sqrt{5.3 .14}(t)+2$, by Lemma 5.3.14 $M^{\prime}$ and hence $M$ has a rank- $t$ double wheel minor. 


\section{Chapter 6}

\section{Blocking $M^{*}\left(K_{3, n}\right)$}

In this chapter we prove the following theorem.

Theorem 6.0.1. There is a function $\sqrt{6.0 .1}$ such that the following hold. Suppose $M$ is a binary matroid and $X$ a coindependent set in $M$ such that $M \backslash X \cong M^{*}\left(K_{3, n}\right)$ where $n \geq 6$ 6.0.1 $(t)$. If every 3-separation of $M \backslash X$ displayed by the canonical flower of $M \backslash X$ is blocked by some element $x \in X$, then $M$ has a minor isomorphic to one of the following matroids.
i) A rank-t circular ladder,
ii) a rank-t Möbius ladder,

iii) a rank-t double wheel,

iv) $\left(N\left(K_{3, t}\right)\right)^{*}$,

v) $M^{*}\left(K_{3, n}\right)$ blocked in a path-like way.

In the first copy of this thesis we believed that we had solved this case when the matroid has no spike minor. However, sadly there was a mistake in the proof of this theorem and instead we have Theorem ?? 
Recall that a standard representation for $M^{*}\left(K_{3, n}\right)$ is a representation of the form

$$
\begin{aligned}
& \begin{array}{llllllll}
c_{1} & c_{2} & c_{3} & \ldots & c_{n-1} & a_{n} & b_{n} & c_{n}
\end{array} \\
& \begin{array}{l}
a_{1} \\
b_{1} \\
a_{2} \\
b_{2} \\
a_{3} \\
b_{3} \\
\vdots \\
a_{n-1} \\
b_{n-1}
\end{array}\left(\begin{array}{cccccccc}
1 & 0 & 0 & \ldots & 0 & 1 & 0 & 1 \\
1 & 0 & 0 & \ldots & 0 & 0 & 1 & 1 \\
0 & 1 & 0 & \ldots & 0 & 1 & 0 & 1 \\
0 & 1 & 0 & \ldots & 0 & 0 & 1 & 1 \\
0 & 0 & 1 & \ldots & 0 & 1 & 0 & 1 \\
0 & 0 & 1 & \ldots & 0 & 0 & 1 & 1 \\
0 & 0 & \vdots & \ddots & \vdots & \vdots & \vdots & \vdots \\
& 0 & 0 & \ldots & 1 & 0 & 1 & 1
\end{array}\right)
\end{aligned}
$$

and a standard basis for $M^{*}\left(K_{3, n}\right)$ is a basis that gives a representation of this form.

In this chapter we work under the hypotheses of Theorem 6.0.1. We also take this opportunity to give some notation local to this chapter. This means that throughout this chapter we work under the following hypotheses.

- $M$ is a matroid and $X$ a coindependent set in $M$ such that $M \backslash X \cong M^{*}\left(K_{3, n}\right)$.

- every 3-separation of $M \backslash X$ displayed by the canonical flower of $M \backslash X$ is blocked by some element $x \in X$ and $X$ is minimal with respect to this property.

- $B=\left\{a_{1}, b_{1}, \ldots, a_{n-1}, b_{n-1}\right\}$ is a standard basis for $M \backslash X$.

- $M$ is represented by the binary matrix $\Gamma$ with respect to a standard basis $B$.

- $\Delta$ is the matrix representing $M \backslash X$ with respect to basis $B$.

- $\Lambda$ is the $2(n-1) \times|X|$ matrix that is the restriction of $A$ to the columns labelled by elements of $X$.

- $\widetilde{\Lambda}$ denotes the $(n-1) \times|X|$ matrix where $\widetilde{\Lambda}_{i, j}=\left(\Lambda_{2 i, j}, \Lambda_{2 i+1, j}\right)^{T}$.

- $\widetilde{\Gamma}$ denotes the $(n-1) \times(n+3+|X|)$ matrix where $\widetilde{\Gamma}_{i, j}=\left(\Gamma_{2 i, j}, \Gamma_{2 i+1, j}\right)^{T}$.

We call the elements in $X$ the blocking elements of $M$. 
The proof of the following lemma is geometrically obvious in the dual ${ }^{1}$ and is omitted.

Lemma 6.0.2. If $n \geq 4^{t}$ then $|X| \geq t$.

This chapter splits into five main sections. In the first section we build a crossing graph for $X$ with respect to $M \backslash X$ and show that this graph must be connected. This section follows [7] very closely. There are three unavoidable induced subgraphs of a simple connected graph, they are a path, a star and a complete graph. The next three sections are dedicated to analyzing these three cases. The final section brings the results of this chapter together in a proof of Theorem 6.0.1.

\subsection{Crossing Graphs}

Definition 6.1.1. Let $\Phi$ be a matrix which takes entries from a set $U$ and let $j$ and $k$ be columns of $P h i$

i) We say that $j$ dominates $k$ in $\Phi$ if $j$ and $k$ are identical or there is some $\alpha$ in $U-\{0\}$ such that whenever $a_{i, k} \neq 0$ we have $a_{i, j}=\alpha$. We use $j \succ k$ to denote the fact that $j$ dominates $k$.

ii) If $j$ dominates $k$ and whenever $a_{i, k} \neq 0$ we have $a_{i, j}=\alpha$ we say that $\alpha$ is the dominating element for the pair $(j, k)$.

iii) We say that $j$ and $k$ cross if neither dominates the other.

Definition 6.1.2. The crossing graph of the matrix $\Phi$ is a graph, $G^{\Phi}$, in which the vertices are labelled by the columns of $\Phi$ and there is an edge between two vertices of $G^{\Phi}$ if, and only if, those two vertices cross as columns of $\Phi$.

The following lemma can be found in [7].

Lemma 6.1.3. Suppose that $G_{0}$ is a connected component of $G^{\Phi}$ for some matrix $\Phi$, and that $k_{0}$ is an element of $V\left(G^{\Phi}\right)-V\left(G_{0}\right)$ that dominates at least one element of $V\left(G_{0}\right)$. Then $k_{0}$ dominates every element of $V\left(G_{0}\right)$.

\footnotetext{
${ }^{1}$ When we coextend by an element $x$ the guts of the paddle goes from being a line to being a plane. Since the matroids we are considering are binary this plane consists of seven lines. Three of these lines contain $x$, the remaining 4 split the paddle into four classes
} 
For the remainder of this section we work under the following hypotheses.

- All matrices take their entries from the set $\left\{(0,0)^{T},(1,0)^{T},(0,1)^{T},(1,1)^{T}\right\}$ unless otherwise stated. Operations on these elements are just the normal vector operations. We use 0 to denote the element $(0,0)^{T}$.

- All columns of $\widetilde{\Lambda}$ are distinct.

Definition 6.1.4. Let $j$ be a column of a matrix $\Phi$. The support of $j$, denoted $s(j)$, is the set of rows that contain a non-zero element in $j$. If $C$ is a set of columns the the support of $C$, denoted $s(C)$, is the union of the supports of the columns of $C$.

We use $t(c)$ to denote the number of elements of the set $\left\{(1,1)^{T},(1,0)^{T},(0,1)^{T},(0,0)^{T}\right\}$ that are used in column $c$ of $\widetilde{\Lambda}$.

The following theorem is very similar to Theorem 4.2 of [7].

Theorem 6.1.5. The crossing graph $G^{\widetilde{\Lambda}}$ is connected.

Proof. Suppose not. Then we can choose a component $G_{0}$ of $G^{\widetilde{\Lambda}}$ according to the following:

1. If $G_{1}$ is a component of $G^{\widetilde{\Lambda}}$ then $s\left(V\left(G_{0}\right)\right) \subseteq s\left(V\left(G_{1}\right)\right.$.

2. If $G_{1}$ is a component of $G^{\widetilde{\Lambda}}$ and $s\left(V\left(G_{0}\right)\right)=s\left(V\left(G_{1}\right)\right)$ then $\left|V\left(G_{1}\right)\right| \leq\left|V\left(G_{0}\right)\right|$.

3. If $G_{1}$ is a component of $G^{\widetilde{\Lambda}}$ and $s\left(V\left(G_{0}\right)\right)=s\left(V\left(G_{1}\right)\right)$ and $\left|V\left(G_{1}\right)\right|=$ $\left|V\left(G_{0}\right)\right|$ then $t\left(V\left(G_{1}\right)\right) \leq t\left(V\left(G_{0}\right)\right)$.

Claim 6.1.6. If $j_{1} \in\left(V\left(G^{\widetilde{\Lambda}}\right)-V\left(G_{0}\right)\right)$ and $j_{0} \in V\left(G_{0}\right)$ are such that $s\left(j_{0}\right) \cap$ $s\left(j_{1}\right) \neq \emptyset$, then $j_{1}$ dominates $j_{0}$.

Proof. As $j_{0}$ and $j_{1}$ are in different components of $G^{\widetilde{\Lambda}}$ we know that they do not cross. Therefore it is sufficient to prove that $j_{0}$ does not dominate $j_{1}$. Suppose for contradiction that $j_{0}$ does dominate $j_{1}$. By Lemma 6.1.3 this means that $j_{0}$ dominates all elements of $G_{1}$. This means that $s\left(V\left(G_{1}\right)\right) \subseteq s\left(j_{0}\right) \subseteq s\left(V\left(G_{0}\right)\right)$ which, by the choice of $G_{0}$ means that $s\left(V\left(G_{1}\right)\right)=s\left(j_{0}\right)=s\left(V\left(G_{0}\right)\right)$. Suppose 
that the entries of $j_{0}$ take more than one non-zero value. Then if $\left|G_{0}\right| \neq 1$ we must have another column of $\widetilde{\Lambda}$ in $G_{0}$ that is a relabelling of $j_{0}$, as otherwise this would not cross $j_{0}$ or would cross an element of $G^{\widetilde{\Lambda}}-G_{0}$. This is a contradiction so in this case $\left|G_{0}\right|=1$. If $j_{0}$ uses only one field element then, as $s\left(j_{0}\right)=s\left(V\left(G_{1}\right)\right)$, $V\left(G_{0}\right)=j_{0}$. By 2 this means that $G_{1}=\left\{j_{1}\right\}$ and so as $s\left(j_{0}\right)=s\left(j_{1}\right)$ we have a contradiction.

Claim 6.1.7. If $j \in V\left(G^{\widetilde{\Lambda}}\right)-V\left(G_{0}\right)$ then there is some element $\alpha$ such that $a_{i, j}=\alpha$ for $i \in s\left(V\left(G_{0}\right)\right)$

Proof. Let $j_{1}$ be an element in $V\left(G^{\widetilde{\Lambda}}\right)-V\left(G_{0}\right)$ that provides a counterexample. Clearly $s\left(j_{1}\right) \cap s\left(V\left(G_{0}\right)\right) \neq \emptyset$ so $s\left(j_{0}\right) \cap s\left(j_{1}\right) \neq \emptyset$ for some $j_{0} \in G_{0}$. By the previous claim this means that $j_{1}$ dominates all elements of $G_{0}$ which contradicts our choice of $j_{1}$.

We can view $s\left(V\left(G_{0}\right)\right)$ as a subset of pairs of columns $c_{2 i-1}, c_{2 i}$ of the identity matrix $I$. Define $X \subseteq E(M)$ so that $X=V\left(G_{0}\right) \cup s\left(V\left(G_{0}\right)\right)$. This has size at least two. Now $E(M)-X$ also has size at least two because it contains an element from $G^{\widetilde{\Lambda}}-G_{0}$ and also contains the columns $(1 \ldots 1),(10 \ldots 10),(01 \ldots 01)$, It is clear that $r(X)=\mid s\left(V\left(G_{0}\right) \mid\right.$ by the above claim it is also clear that $(I-$ $\left.s\left(V\left(G_{0}\right)\right) \cup\left\{(\alpha \ldots \alpha)^{T}\right\}\right)$ spans $E(M)-X$ where $\alpha=(1,0),(0,1)$ or $(1,1)$. Therefore, $r(E(M)-X) \leq r\left(I-s\left(V\left(G_{0}\right)\right) \cup(\alpha \ldots \alpha)^{T}\right) \leq|I|-|s(V(G))|+1=r(M)-$ $r(X)+1$ Therefore $(x, E-X)$ is a 2-separation.

Since $G^{\widetilde{\Lambda}}$ is connected it follows, by Lemma 2.4.2, that we can find an induced subgraph that is either a star, a complete graph or a path.

Definition 6.1.8. The crossing graph of $\widetilde{\Lambda}$ is the crossing graph of $X$ in $M$.

Theorem 6.1.9. There is a function 6 such that the following holds. Suppose $M$ is a binary matroid such that $M \backslash X \cong M^{*}\left(K_{3, n}\right)$, and $X$ blocks all 3-separations of $M \backslash X$ displayed by the canonical flower of $M \backslash X$. If $n \geq f[6.1 .9(t)$, then there is a minor $M^{\prime}$ of $M$ with the following properties.

1. $M^{\prime} \backslash\left(X \cap E\left(M^{\prime}\right)\right) \cong M^{*}\left(K_{3, t}\right)$,

2. every 3-separation of $M \backslash X$ displayed by the canonical flower of $M \backslash X$ is blocked by an element of $X$, and 
3. the crossing graph of $X^{\prime}$ in $M^{\prime}$ is either a star, a path or a complete graph.

Proof. Suppose $n \geq \sqrt{2.4 .2}\left(\log _{4}(t)\right)$. Consider the crossing graph, $G^{\widetilde{\Lambda}}$, of $X$ in $M$. The graph $G^{\widetilde{\Lambda}}$ has an induced subgraph $G_{0}$ with at least $\log _{4}(t)$ vertices and let the vertex set of $G_{0}$ be $X^{\prime}$. Consider $\widetilde{\Gamma} \mid X^{\prime}$. Since all columns of $X^{\prime}$ are distinct, this must have at least $t$ rows which, for some column of $\widetilde{\Gamma} \mid X^{\prime}$, are non-zero. Now consider $\widetilde{\Gamma}$, and delete all columns labelled by elements of $X-X^{\prime}$, and delete all rows in which no column of $\widetilde{\Gamma} \mid X^{\prime}$ contains a non-zero entry. Finally if the $i^{\text {th }}$ row of $\widetilde{\Gamma}$ is deleted, also delete the $i^{t h}$ column. Call the matrix obtained in this way $\widetilde{\Gamma_{0}}$ and note that $\widetilde{\Gamma_{0}}$ has at least $t$ rows. Let $\Gamma_{0}$ be the matrix obtained by considering each element of $\widetilde{\Gamma_{0}}$ to be two elements in the natural way. The matroid with reduced standard representation given by $\Gamma_{0}$ fulfills the requirements for $M^{\prime}$ given in the statement of the Theorem.

\subsection{Complete Graph}

In this section we prove the following theorem.

Theorem 6.2.1. There is a function 6 6.2.1 such that the following holds. Suppose $M$ is a binary matroid with a coindependent set $X$ such that $M \backslash X \cong M^{*}\left(K_{3, n}\right)$, that $X$ is such that every 3-separation displayed by the canonical flower of $M \backslash X$ is blocked by an element of $X$, and that the crossing graph of $X$ in $M$ is a complete graph. If $n \geq \sqrt{6.2 .1}(t)$ then $M$ has a minor isomorphic to one of the following:

1. a rank-t Möbius ladder,

2. a rank-t double wheel, or

3. $N\left(K_{3, t}\right)^{*}$.

In this section we work under the hypotheses of Theorem 6.2.1. That is we add to our original hypotheses the following hypothesis.

- The crossing graph of $X$ with respect to $M \backslash X$ is a complete graph. 
Definition 6.2.2. A square matrix, $A$, is $(\alpha, \beta, \gamma)$-diagonal if

$$
A_{i, j}= \begin{cases}\alpha, & \text { if } i<j \\ \beta, & \text { if } i=j \\ \gamma & \text { if } i>j\end{cases}
$$

A $5 \times 5$ example of an $(\alpha, \beta, \gamma)$-diagonal matrix is the following:

$$
\left(\begin{array}{lllll}
\beta & \alpha & \alpha & \alpha & \alpha \\
\gamma & \beta & \alpha & \alpha & \alpha \\
\gamma & \gamma & \beta & \alpha & \alpha \\
\gamma & \gamma & \gamma & \beta & \alpha \\
\gamma & \gamma & \gamma & \gamma & \beta
\end{array}\right)
$$

We say that a matrix $M$ is $(\alpha, \beta)$-diagonal if $M$ is $(\alpha, \alpha, \beta)$-diagonal.

Definition 6.2.3. Let $H$ be a finite field. A matrix $A$ taking entries from $H$ is $(\alpha, \beta)$ - complete if the number of rows of $A$ is $\left(\begin{array}{l}n \\ 2\end{array}\right)$, where $\mathrm{n}$ is the number of columns of $A$, and, for every two distinct columns $j$ and $j^{\prime}$ of $A$, there is exactly one row $i$ of $A$ such that $A_{i, \min \left\{j, j^{\prime}\right\}}$ and $A_{i, \max \left\{j, j^{\prime}\right\}}$ are $\alpha$ and $\beta$ respectively, and $A_{i, k}=0$ for all $k \notin\left\{j, j^{\prime}\right\}$

A $4 \times 6$ example of an $(\alpha, \beta)$-complete matrix is:

$$
\left(\begin{array}{llll}
\alpha & \beta & 0 & 0 \\
\alpha & 0 & \beta & 0 \\
\alpha & 0 & 0 & \beta \\
0 & \alpha & \beta & 0 \\
0 & \alpha & 0 & \beta \\
0 & 0 & \alpha & \beta
\end{array}\right)
$$

The next theorem is Theorem 2.7 of [7].

Theorem 6.2.4. There is a function 6 6.2.4 with the following property. If $t$ is an integer greater than one and $A$ is an F-matrix with at least $f 6.2 .4(t)$ columns such that every two columns of A cross, then A contains a row and column permuted submatrix $B$ with t columns that satisfies one of the following conditions: 
i) B has $t+1$ rows the first $t$ of which form an $(\alpha, \alpha, 0)$-diagonal matrix and the last of which has all its entries equal to $\beta$ for some $\beta \in F-\{0, \alpha\}$,

ii) $B$ has $2 t$ rows the first $t$ of which form a $(0, \alpha, \alpha)$-diagonal matrix and the last $t$ of which form an $(\alpha, \alpha, 0)$-diagonal matrix,

iii) $B$ has $t$ rows and is $(\alpha, \beta, \gamma)$-diagonal with $\alpha \neq \beta, \alpha \neq 0$ and $\gamma \neq 0$,

iv) $B$ has $t+1$ rows the first of which form a $(0, \alpha, 0)$-diagonal matrix and the last of which has all entries equal to some non-zero $\beta$,

v) $B$ is $(\alpha, \beta)$-complete for some nonzero elements $\alpha$ and $\beta$ of $F$,

where, for all the above cases, $\alpha \neq 0$. 
The following lemma is trivial.

Lemma 6.2.5. Consider the matrix $\Gamma$ where $\widetilde{\Lambda}$ is of one of the forms described in Lemma 6.2.4 and $\alpha, \beta, \gamma \in\left\{(1,1)^{T},(1,0)^{T},(0,1)^{T},(0,0)^{T}\right\}$. If $\alpha \neq(0,0)^{T}$ then we can always perform a change of basis to obtain a matrix $\Gamma_{1}$ that is a reduced standard representation of $M$ such that $\Gamma_{1} \mid(E-X)$ is a standard representation of $M^{*}\left(K_{3, n}\right)$, and $\Gamma_{1} \mid X$ is of the same form from Lemma 6.2.4 as $\Gamma \mid X$, but $\alpha$ is a choice of $\left\{(1,1)^{T},(1,0)^{T},(0,1)^{T}\right\}$.

Lemma 6.2.6. There is a function 6 6.2.6 such the following holds. If $M$ is such that $\widetilde{\Lambda}$ is of the form of i) from Lemma 6.2.4 and $n \geq 6$ 6.2.6 $(t)$, then $M$ has a Möbius ladder of rank at least $t$ as a minor.

Proof. By Lemma 6.2.5 we may assume that $\alpha=(1,1)^{T}$. Therefore $\beta=(1,0)^{T}$ or $(0,1)^{T}$. Without loss of generality let $\beta=(1,0)^{T}$. Let $n=t+1$. We can represent $M$ by the following reduced standard representation matrix:

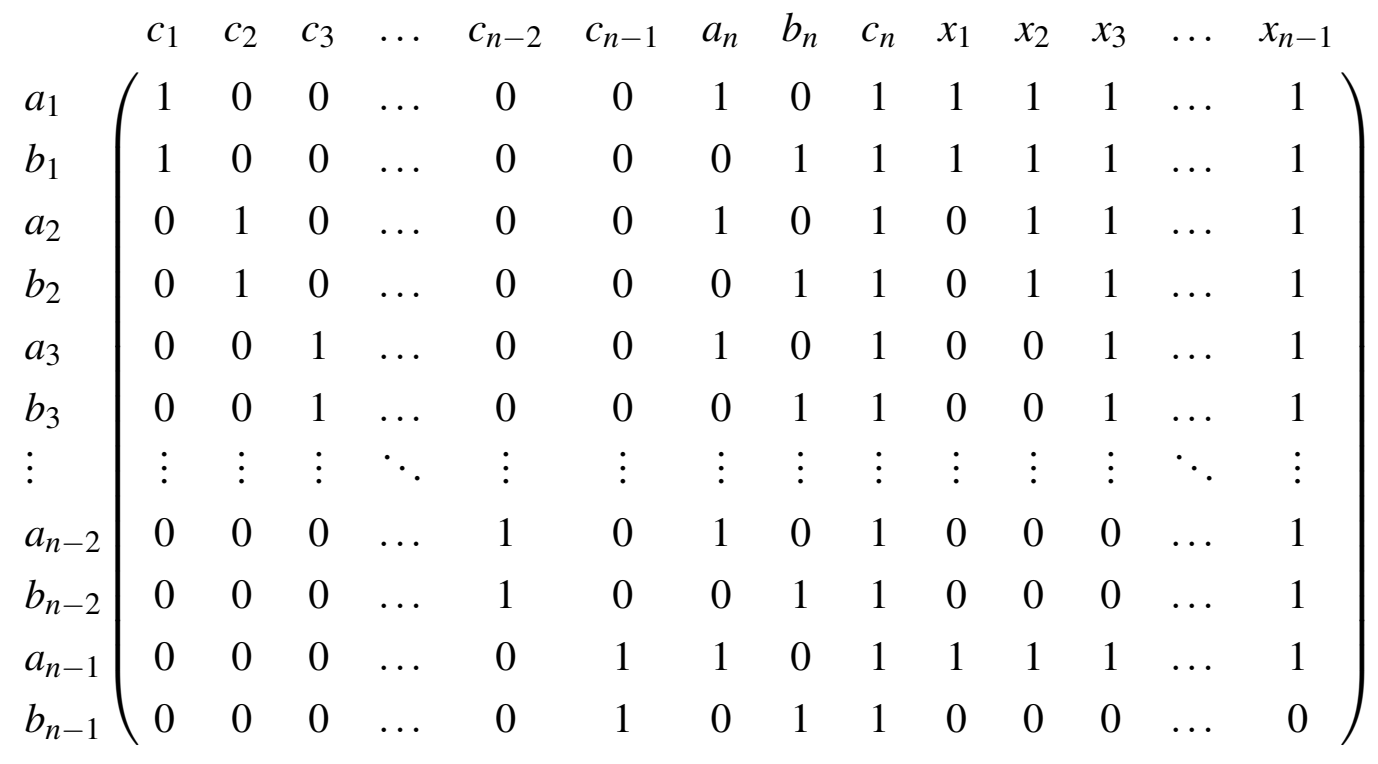

Consider the reduced standard representation matrix of $M \backslash\left\{c_{1}, \ldots, c_{n-2}, c_{n}\right\}$ below. 


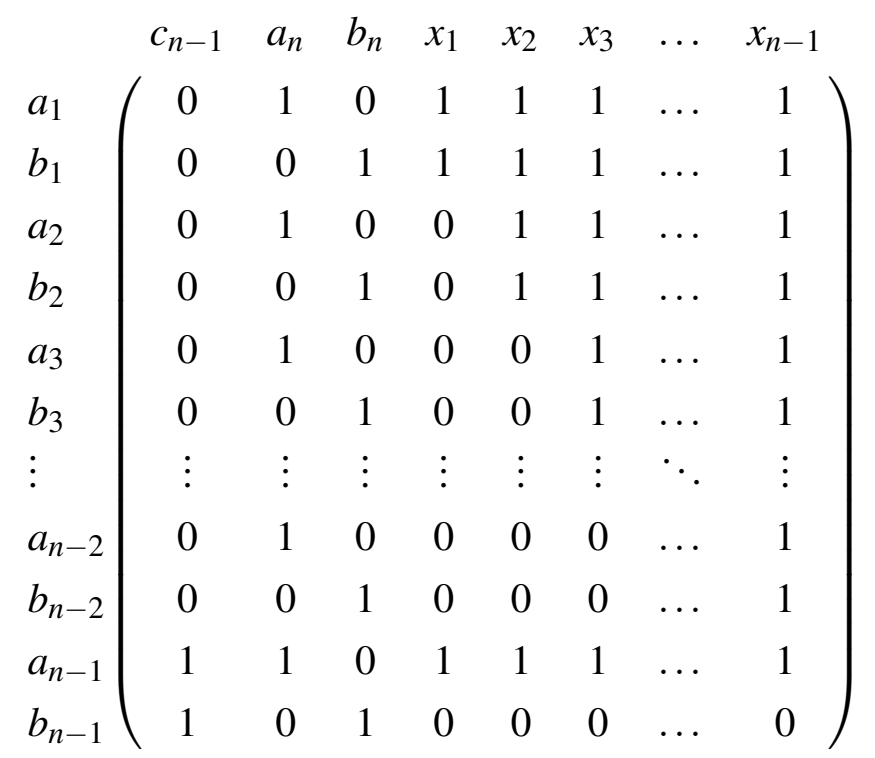

Pivot on $M_{b_{n-1}, c_{n-1}}$ to get:

$$
\begin{aligned}
& \begin{array}{llllllll}
b_{n-1} & a_{n} & b_{n} & x_{1} & x_{2} & x_{3} & \ldots & x_{n-1}
\end{array}
\end{aligned}
$$

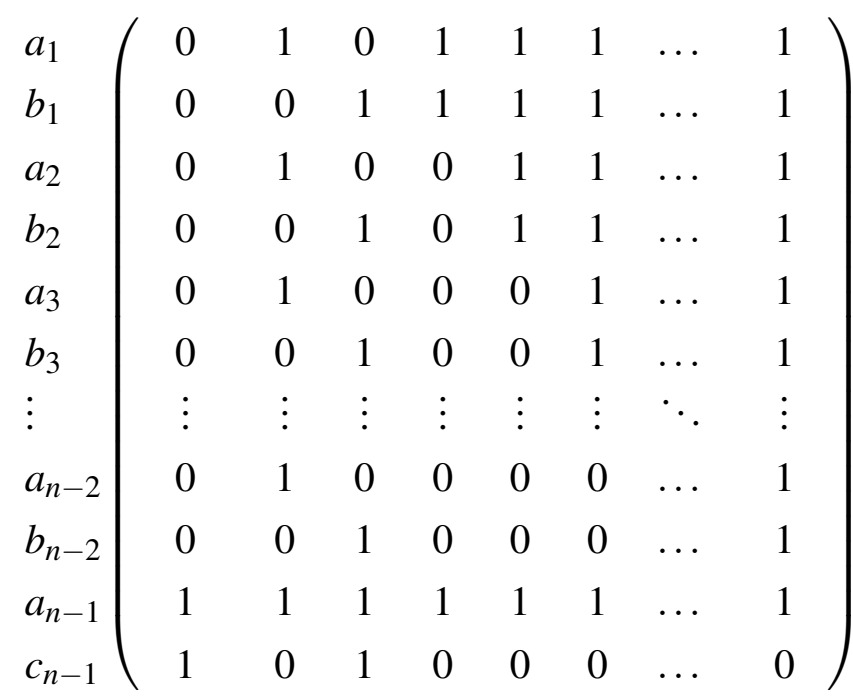

It is then easy to see that when we contract $c_{n-1}$ and delete $b_{n-1}$ the resulting matroid is a Möbius ladder and has rank $t$. 
We now consider the case where $\widetilde{\Lambda}$ is of form ii) from Lemma 6.2.4. A $6 \times 3$ example of a matrix of form ii) of Lemma 6.2.4 is the following:

$$
\left(\begin{array}{lll}
\alpha & 0 & 0 \\
\alpha & \alpha & 0 \\
\alpha & \alpha & \alpha \\
\alpha & \alpha & \alpha \\
0 & \alpha & \alpha \\
0 & 0 & \alpha
\end{array}\right)
$$

Lemma 6.2.7. There is a function 6 6.2.7 such that the following holds. If $\widetilde{D}$ is of form ii) from Lemma 6.2.4 and $n \geq 6$ 6.2.7 $(t)$, then $M$ has a Möbius ladder of rank at least $t$ as a minor.

Proof. Suppose $n \geq 2 m+1$ where

$$
t= \begin{cases}2 m & \text { if } t \text { is even } \\ 2 m-1 & \text { if } t \text { is odd }\end{cases}
$$

The matroid $M$ can be represented by the matrix on the following page. 


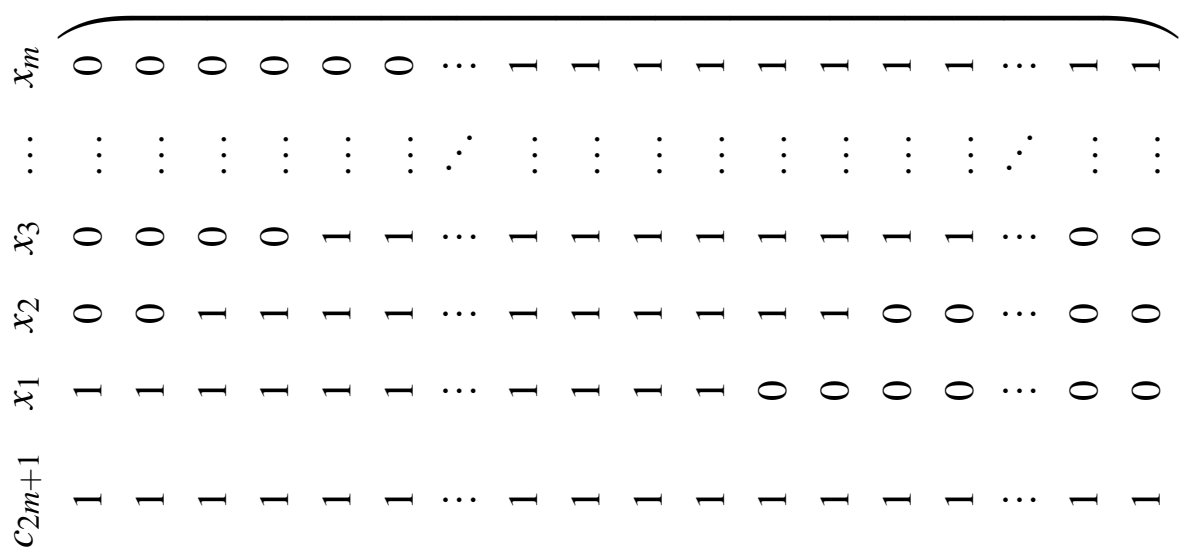

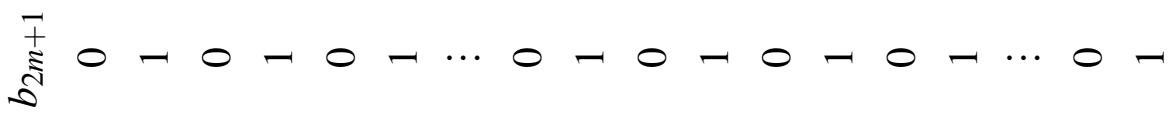

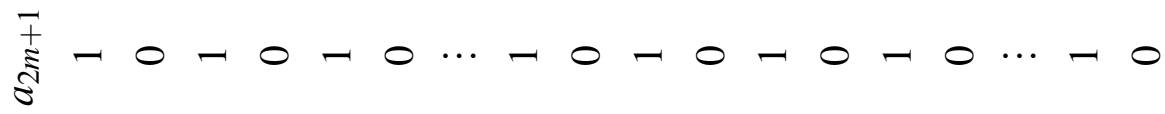

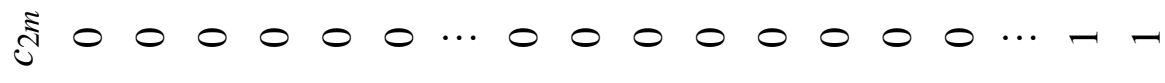
$\begin{array}{lllllllllllllllllll}\vdots & \vdots & \vdots & \vdots & \vdots & \vdots & \vdots & \ddots & \vdots & \vdots & \vdots & \vdots & \vdots & \vdots & \vdots & \vdots & \ddots & \vdots & \vdots\end{array}$

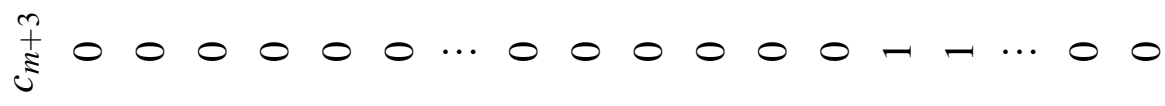
I I

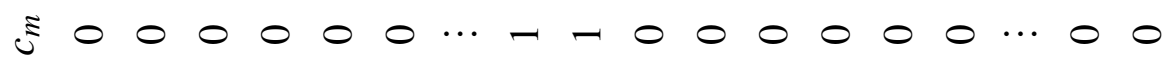

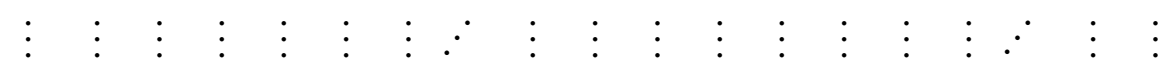

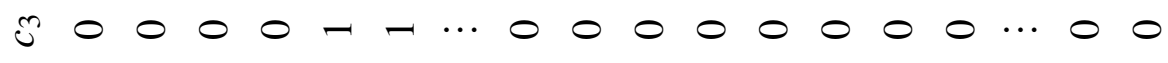
N

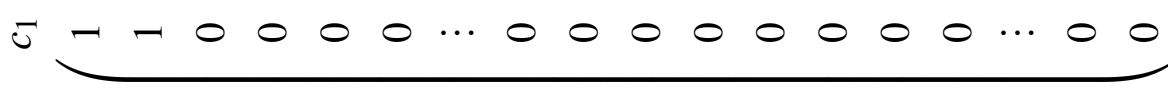

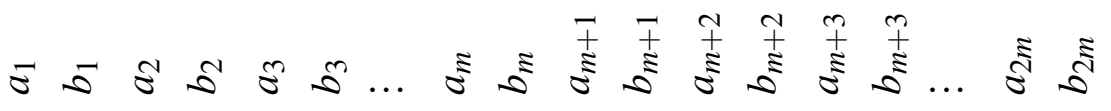


Deleting $c_{1}, \ldots, c_{2 m}, a_{2 m+1}, b_{2 m+1}$ and contracting $a_{1}, \ldots, a_{2 m}$ then gives the following matrix:

$$
\begin{aligned}
& \begin{array}{llllll}
c_{2 m+1} & x_{1} & x_{2} & x_{3} & \ldots & x_{m}
\end{array} \\
& \begin{array}{l}
b_{1} \\
b_{2} \\
b_{3} \\
\vdots \\
b_{m+1} \\
b_{m+2} \\
b_{m+3} \\
\vdots \\
b_{2 m}
\end{array}\left(\begin{array}{cccccc}
1 & 1 & 0 & 0 & \ldots & 0 \\
1 & 1 & 1 & 0 & \ldots & 0 \\
1 & 1 & 1 & 1 & \ldots & 0 \\
1 & \vdots & \vdots & \vdots & \ddots & \vdots \\
1 & 0 & 1 & 1 & \ldots & 1 \\
\vdots & 0 & 0 & 1 & \ldots & 1 \\
1 & 0 & 0 & 0 & \ldots & 1
\end{array}\right)
\end{aligned}
$$

which is a representation of a Möbius ladder of rank $2 m$ and therefore rank at least $t$.

Lemma 6.2.8. There is a function $\sqrt{6.2 .8}$ such that the following holds. Suppose $\widetilde{\Lambda}$ is a matrix of blocking elements of form iv) from Lemma 6.2.4. If $n \geq 6$ 6.2.8 $(t)$, then $M$ has a $\left(N\left(K_{3, t}\right)^{*}\right.$-minor.

Proof. Let $n \geq \sqrt{5.2 .5}(t)+3$. By performing a change of basis we may split this into two cases

a) $\alpha=(1,1)$ and $\beta=(1,1)$

b) $\alpha=(1,1)$ and $\beta=(1,0)$ 
This can be represented by the following matrix:

\begin{tabular}{|c|c|c|c|c|c|c|c|c|c|c|c|c|c|}
\hline & $c_{1}$ & $c_{2}$ & $c_{3}$ & 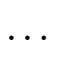 & $c_{n-2}$ & $c_{n-1}$ & $a_{n}$ & $b_{n}$ & $c_{n}$ & $x_{1}$ & $x_{2}$ & .. & $x_{n-2}$ \\
\hline$a_{1}$ & 1 & 0 & 0 & 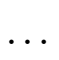 & 0 & 0 & 1 & 0 & 1 & 1 & 1 & 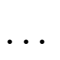 & 1 \\
\hline$b_{1}$ & 1 & 0 & 0 & .. & 0 & 0 & 0 & 1 & 1 & $? 0$ & $?$ & 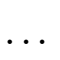 & $?$ \\
\hline$a_{2}$ & 0 & 1 & 0 & $\cdots$ & 0 & 0 & 1 & 0 & 1 & 1 & 0 & $\ldots$ & 0 \\
\hline$b_{2}$ & 0 & 1 & 0 & 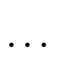 & 0 & 0 & 0 & 1 & 1 & 1 & 0 & . & 0 \\
\hline$a_{3}$ & 0 & 0 & 1 & $\cdots$ & 0 & 0 & 1 & 0 & 1 & 0 & 1 & $\cdots$ & 0 \\
\hline$b_{3}$ & 0 & 0 & 1 & $\ldots$ & 0 & 0 & 0 & 1 & 1 & 0 & 1 & 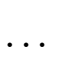 & 0 \\
\hline$\vdots$ & $\vdots$ & $\vdots$ & $\vdots$ & $\ddots$ & $\vdots$ & $\vdots$ & $\vdots$ & $\vdots$ & $\vdots$ & $\vdots$ & ${ }^{\circ}$ & $\vdots$ & \\
\hline$a_{n-1}$ & 0 & 0 & 0 & $\ldots$ & 0 & 1 & 1 & 0 & 1 & 0 & 0 & 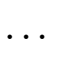 & 1 \\
\hline$b_{n-1}$ & 0 & 0 & 0 & $\cdots$ & 0 & 1 & 0 & 1 & 1 & 0 & 0 & & 1 \\
\hline
\end{tabular}

Delete $c_{1}, \ldots, c_{n}$ and contract $b_{1}, a_{2}, b_{2}$ to get:

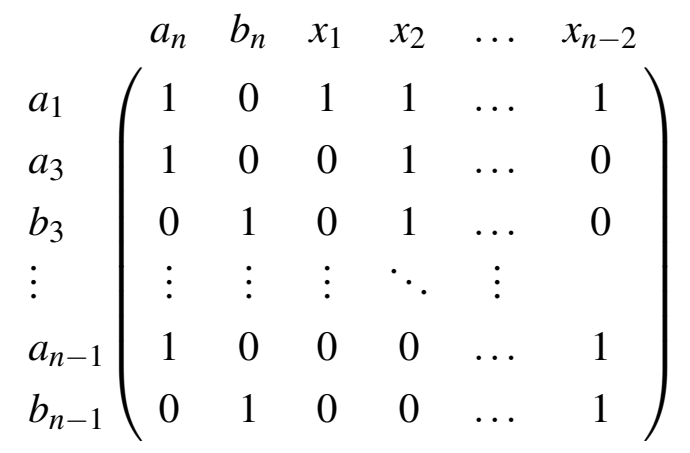

which is the dual of case $\left(a^{\prime}\right)$ from Lemma 5.2.1 for the matrix $M\left(K_{3, m}\right)$ where $m=f_{5.2 .5}(t)$. Therefore, by the dual of Theorem 5.2.5, $M$ has a $N\left(K_{3, t}\right)^{*}$-minor.

Lemma 6.2.9. There is a function 6 6.2.9 such that the following holds. Suppose $\widetilde{\Lambda}$ is ofform iii) from Lemma 6.2.4. If $n \geq \sqrt{6.2 .9}(t)$ then M either has a $N\left(K_{3, t}\right)$-minor or a Möbius ladder of rank at least $t$ as a minor.

Proof. Suppose $n \geq \max \{\sqrt{6.2 .8}(t)+1,2 \sqrt{6.2 .6}(t)\}=m$.

If $\alpha \neq \gamma$ then without loss of generality let $\alpha=(1,1)^{T}$ and $\gamma=(1,0)^{T}$. Let $m \geq 2 m^{\prime}$ for some $m^{\prime} \geq \sqrt{6.2 .6}(t)$. There is a minor $M^{\prime}$ of $M$ obtained by contracting $a_{i}, b_{i}$ when $i$ is even and deleting $c_{i}, x_{i}$ when $i$ is odd for all $i \in\{1, \ldots, m-1\}$. Let $X^{\prime}=\left\{x_{2}, x_{4}, \ldots, x_{2 m^{\prime}}\right.$. The matrix $M^{\prime} \mid X^{\prime}$ is $(\alpha, \beta)$-diagonal. After relabelling we 
get the following representation of $M^{\prime}$

\begin{tabular}{|c|c|c|c|c|c|c|c|c|c|c|c|c|c|c|}
\hline & $c_{1}$ & $c_{2}$ & $c_{3}$ & $\ldots$ & $c_{m-2}$ & $c_{m-1}$ & $a_{m}$ & $b_{m}$ & $c_{m}$ & $x_{1}$ & $x_{2}$ & $x_{3}$ & $\ldots$ & $x_{m-1}$ \\
\hline$a_{1}$ & 1 & 0 & 0 & $\ldots$ & 0 & 0 & 1 & 0 & 1 & 1 & 1 & 1 & $\ldots$ & 1 \\
\hline$b_{1}$ & 1 & 0 & 0 & . & 0 & 0 & 0 & 1 & 1 & 1 & 1 & 1 & 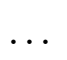 & 1 \\
\hline$a_{2}$ & 0 & 1 & 0 & $\ldots$ & 0 & 0 & 1 & 0 & 1 & 1 & 1 & 1 & & 1 \\
\hline$b_{2}$ & 0 & 1 & 0 & 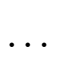 & 0 & 0 & 0 & 1 & 1 & 0 & 1 & 1 & & 1 \\
\hline$a_{3}$ & 0 & 0 & 1 & $\ldots$ & 0 & 0 & 1 & 0 & 1 & 1 & 1 & 1 &. & 1 \\
\hline$b_{3}$ & 0 & 0 & 1 & $\ldots$ & 0 & 0 & 0 & 1 & 1 & 0 & 0 & 1 & $\ldots$ & 1 \\
\hline$\vdots$ & $\vdots$ & $\vdots$ & $\vdots$ & $\ddots$ & $\vdots$ & $\vdots$ & $\vdots$ & $\vdots$ & $\vdots$ & $\vdots$ & $\vdots$ & $\vdots$ & $\ddots$. & $\vdots$ \\
\hline$a_{m-2}$ & 0 & 0 & 0 & $\ldots$ & 1 & 0 & 1 & 0 & 1 & 1 & 1 & 1 & & 1 \\
\hline$b_{m-2}$ & 0 & 0 & 0 & $\ldots$ & 1 & 0 & 0 & 1 & 1 & 0 & 0 & 0 & 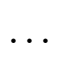 & 1 \\
\hline$a_{m-1}$ & 0 & 0 & 0 & $\ldots$ & 0 & 1 & 1 & 0 & 1 & 1 & 1 & 1 &. & 1 \\
\hline$b_{m-1}$ & 0 & 0 & 0 & $\ldots$ & 0 & 1 & 0 & 1 & 1 & 0 & 0 & 0 & & 0 \\
\hline
\end{tabular}

Pivoting on $M_{a_{1}, a_{m}}$ gives

\begin{tabular}{|c|c|c|c|c|c|c|c|c|c|c|c|c|c|c|}
\hline & $c_{1}$ & $c_{2}$ & $c_{3}$ & $\ldots$ & $c_{m-2}$ & $c_{m-1}$ & $a_{1}$ & $b_{m}$ & $c_{m}$ & $x_{1}$ & $x_{2}$ & $x_{3}$ & $\ldots$ & $x_{m-1}$ \\
\hline$a_{m}$ & ( 1 & 0 & 0 & $\ldots$ & 0 & 0 & 1 & 0 & 1 & 1 & 1 & 1 & $\ldots$ & 1 \\
\hline$b_{1}$ & 1 & 0 & 0 & $\ldots$ & 0 & 0 & 0 & 1 & 1 & 1 & 1 & 1 & $\ldots$ & 1 \\
\hline$a_{2}$ & 1 & 1 & 0 & $\ldots$ & 0 & 0 & 1 & 0 & 0 & 0 & 0 & 0 & . & 0 \\
\hline$b_{2}$ & 0 & 1 & 0 & $\ldots$ & 0 & 0 & 0 & 1 & 1 & 0 & 0 & 1 & $\ldots$ & 1 \\
\hline$a_{3}$ & 1 & 0 & 1 & $\ldots$ & 0 & 0 & 1 & 0 & 0 & 0 & 0 & 0 & $\ldots$ & 0 \\
\hline$b_{3}$ & 0 & 0 & 1 & $\ldots$ & 0 & 0 & 0 & 1 & 1 & 0 & 0 & 1 & $\ldots$ & 1 \\
\hline$\vdots$ & $\vdots$ & $\vdots$ & $\vdots$ & $\ddots$ & $\vdots$ & $\vdots$ & $\vdots$ & $\vdots$ & $\vdots$ & $\vdots$ & $\vdots$ & $\vdots$ & $\ddots$ & $\vdots$ \\
\hline$a_{m-2}$ & 1 & 0 & 0 & .. & 1 & 0 & 1 & 0 & 0 & 0 & 0 & 0 & & 0 \\
\hline$b_{m-2}$ & 0 & 0 & 0 & $\ldots$ & 1 & 0 & 0 & 1 & 1 & 0 & 0 & 0 & 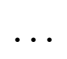 & 1 \\
\hline$a_{m-1}$ & 1 & 0 & 0 & $\ldots$ & 0 & 1 & 1 & 0 & 0 & 0 & 0 & 0 & $\ldots$ & 0 \\
\hline$b_{m-1}$ & 0 & 0 & 0 & $\ldots$ & 0 & 1 & 0 & 1 & 1 & 0 & 0 & 0 & $\ldots$ & 0 \\
\hline
\end{tabular}

Pivoting on $M_{b_{1}, b_{m}}$ then gives 


\begin{tabular}{|c|c|c|c|c|c|c|c|c|c|c|c|c|c|c|}
\hline & $c_{1}$ & $c_{2}$ & $c_{3}$ & $\ldots$ & $c_{m-2}$ & $c_{m-1}$ & $a_{1}$ & $b_{1}$ & $c_{m}$ & $x_{1}$ & $x_{2}$ & $x_{3}$ & $\ldots$ & $x_{m-1}$ \\
\hline$a_{m}$ & 1 & 0 & 0 & $\ldots$ & 0 & 0 & 1 & 0 & 1 & 1 & 1 & 1 & $\ldots$ & 1 \\
\hline$b_{m}$ & 1 & 0 & 0 & $\ldots$ & 0 & 0 & 0 & 1 & 1 & 1 & 1 & 1 & $\ldots$ & 1 \\
\hline$a_{2}$ & 1 & 1 & 0 & $\ldots$ & 0 & 0 & 1 & 0 & 0 & 0 & 0 & 0 & $\ldots$ & 0 \\
\hline$b_{2}$ & 1 & 1 & 0 & $\ldots$ & 0 & 0 & 0 & 1 & 0 & 1 & 0 & 0 & $\ldots$ & 0 \\
\hline$a_{3}$ & 1 & 0 & 1 & $\ldots$ & 0 & 0 & 1 & 0 & 0 & 0 & 0 & 0 & $\ldots$ & 0 \\
\hline$b_{3}$ & 1 & 0 & 1 & $\ldots$ & 0 & 0 & 0 & 1 & 0 & 1 & 1 & 0 & $\ldots$ & 0 \\
\hline$\vdots$ & $\vdots$ & $\vdots$ & $\vdots$ & $\ddots$ & $\vdots$ & $\vdots$ & $\vdots$ & $\vdots$ & $\vdots$ & $\vdots$ & $\vdots$ & $\vdots$ & $\ddots$ & $\vdots$ \\
\hline$a_{m-2}$ & 1 & 0 & 0 & $\cdots$ & 1 & 0 & 1 & 0 & 0 & 0 & 0 & 0 & $\ldots$ & 0 \\
\hline$b_{m-2}$ & 1 & 0 & 0 & $\ldots$ & 1 & 0 & 0 & 1 & 0 & 1 & 1 & 1 & $\ldots$ & 0 \\
\hline$a_{m-1}$ & 1 & 0 & 0 & $\ldots$ & 0 & 1 & 1 & 0 & 0 & 0 & 0 & 0 & $\ldots$ & 0 \\
\hline$b_{m-1}$ & 1 & 0 & 0 & $\ldots$ & 0 & 1 & 0 & 1 & 0 & 1 & 1 & 1 & $\ldots$ & 1 \\
\hline
\end{tabular}

It is clear that by rearranging rows and columns of this matrix we obtain a matrix of the form described in the hypotheses of Lemma 6.2.6. Since $m \geq \sqrt{6.2 .6}(t)$, by Lemma 6.2.6 $M$ has a Möbius ladder of rank at least $t$ as a minor.

Suppose that $\alpha=\gamma$. Without loss of generality let $\alpha=\gamma=(1,1)^{T}$. Up to relabelling we have two choices for $\beta$ that is $\beta=(1,0)^{T}$ or $(0,0)^{T}$. First suppose that $\beta=(1,0)^{T}$. Then $M$ can be represented by:

\begin{tabular}{|c|c|c|c|c|c|c|c|c|c|c|c|c|c|c|}
\hline & $c_{1}$ & $c_{2}$ & $c_{3}$ & $\ldots$ & $c_{m-2}$ & $c_{m-1}$ & $a_{m}$ & $b_{m}$ & $c_{m}$ & $x_{1}$ & $x_{2}$ & $x_{3}$ & $\ldots$ & $x_{m-1}$ \\
\hline$a_{1}$ & 1 & 0 & 0 & $\ldots$ & 0 & 0 & 1 & 0 & 1 & 1 & 1 & 1 & $\ldots$ & 1 \\
\hline$b_{1}$ & 1 & 0 & 0 & $\ldots$ & 0 & 0 & 0 & 1 & 1 & 0 & 1 & 1 & 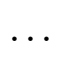 & 1 \\
\hline$a_{2}$ & 0 & 1 & 0 & $\ldots$ & 0 & 0 & 1 & 0 & 1 & 1 & 1 & 1 & .. & 1 \\
\hline$b_{2}$ & 0 & 1 & 0 & $\ldots$ & 0 & 0 & 0 & 1 & 1 & 1 & 0 & 1 & $\ldots$ & 1 \\
\hline$a_{3}$ & 0 & 0 & 1 & $\ldots$ & 0 & 0 & 1 & 0 & 1 & 1 & 1 & 1 & $\ldots$ & 1 \\
\hline$b_{3}$ & 0 & 0 & 1 & $\ldots$ & 0 & 0 & 0 & 1 & 1 & 1 & 1 & 0 & $\ldots$ & 1 \\
\hline : & $\vdots$ & $\vdots$ & $\vdots$ & $\ddots$ & & $\vdots$ & $\vdots$ & $\vdots$ & $\vdots$ & $\vdots$ & $\vdots$ & $\vdots$ & $\vdots$ & $\ddots$ \\
\hline$a_{m-1}$ & 0 & 0 & 0 & $\ldots$ & 0 & 1 & 1 & 0 & 1 & 1 & 1 & 1 & $\ldots$ & 1 \\
\hline$b_{m-1}$ & 0 & 0 & 0 & $\ldots$ & 0 & 1 & 0 & 1 & 1 & 1 & 1 & 1 & $\cdots$ & 0 \\
\hline
\end{tabular}


Pivot on $M_{a_{1}, a_{m}}$ to get:

\begin{tabular}{|c|c|c|c|c|c|c|c|c|c|c|c|c|c|c|}
\hline & $c_{1}$ & $c_{2}$ & $c_{3}$ & $\ldots$ & $c_{m-2}$ & $c_{m-1}$ & $a_{1}$ & $b_{m}$ & $c_{m}$ & $x_{1}$ & $x_{2}$ & $x_{3}$ & $\ldots$ & $x_{m-1}$ \\
\hline$a_{m}$ & $(1$ & 0 & 0 & 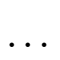 & 0 & 1 & 0 & 1 & 1 & 1 & 1 & $\ldots$ & 1 & \\
\hline$b_{1}$ & 1 & 0 & 0 & $\cdots$ & 0 & 0 & 1 & 1 & 0 & 1 & 1 & $\ldots$ & 1 & \\
\hline$a_{2}$ & 1 & 1 & 0 & $\ldots$ & 0 & 1 & 0 & 0 & 0 & 0 & 0 & $\ldots$ & 0 & \\
\hline$b_{2}$ & 0 & 1 & 0 & $\ldots$ & 0 & 0 & 1 & 1 & 1 & 0 & 1 & $\ldots$ & 1 & \\
\hline$a_{3}$ & 1 & 0 & 1 & $\ldots$ & 0 & 1 & 0 & 0 & 0 & 0 & 0 & $\ldots$ & 0 & \\
\hline$b_{3}$ & 0 & 0 & 1 & .. & 0 & 0 & 0 & 1 & 1 & 1 & 1 & 0 & $\cdots$ & 1 \\
\hline . & $\vdots$ & $\vdots$ & $\vdots$ & $\ddots$ & $\vdots$ & $\vdots$ & $\vdots$ & $\vdots$ & $\vdots$ & $\vdots$ & $\vdots$ & $\ddots$ & $\vdots$ & \\
\hline$a_{m-1}$ & 1 & 0 & 0 & $\ldots$ & 1 & 1 & 0 & 0 & 0 & 0 & 0 & $\ldots$ & 0 & \\
\hline$b_{m-1}$ & 0 & 0 & 0 & $\ldots$ & 1 & 0 & 1 & 1 & 1 & 1 & 1 & $\ldots$ & 0 & \\
\hline
\end{tabular}

Pivot on $M_{b_{1}, b_{m}}$ to get:

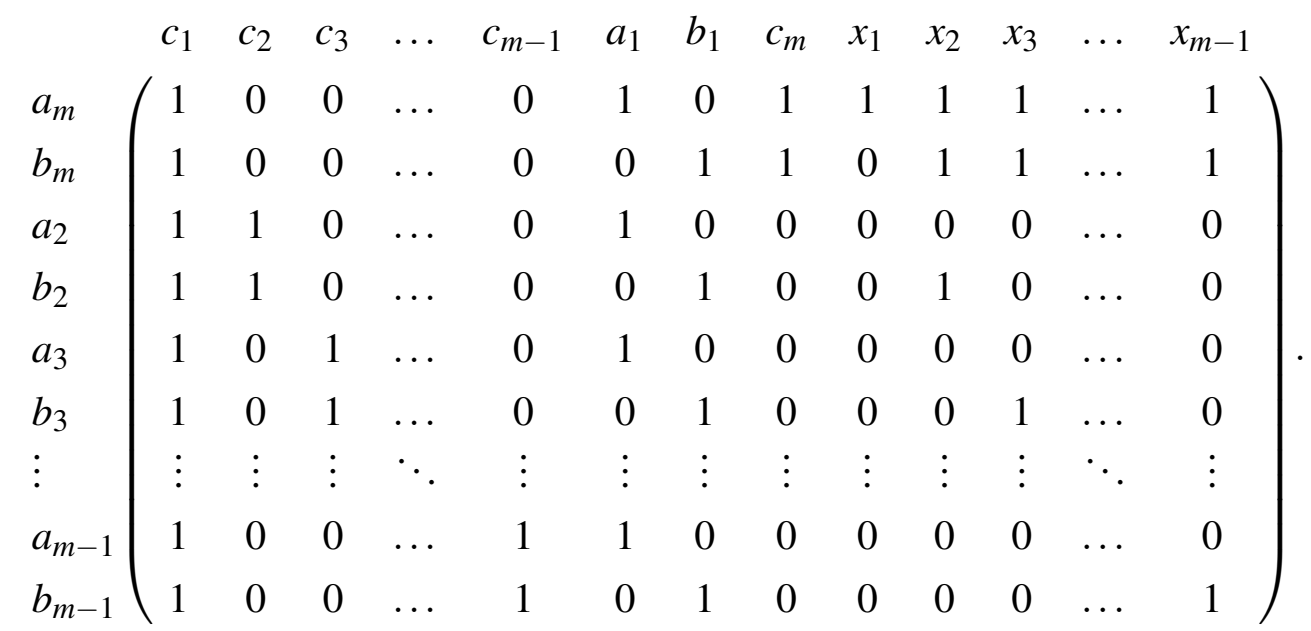

Deleting $c_{m}$ and contracting $a_{m}, b_{m}$ then gives a matroid of the form described in Lemma 6.2.8. Since $m \geq \sqrt[6.2 .8]{ }(t)+1$, it follows from Lemma 6.2.8 that $M$ has a $\left(N\left(K_{3, t}\right)\right)^{*}$-minor. A similar argument works when $\beta=(0,0)^{T}$.

Lemma 6.2.10. There is a function $f_{6.2 .10}$ such that the following holds. Suppose $\widetilde{\Lambda}$ is ofform $v$ ) from Lemma 6.2.4. If $n \geq \sqrt{6.2 .10}(t)$ then $M$ has a double wheel of rank at least $t$ as a minor.

Proof. When $\alpha=(1,1)$ and $\beta=(1,0)$ then $M /\left\{b_{1}, \ldots, b_{m}\right\} \cong M^{*}\left(K_{m}\right)$. The cases where $\beta=(1,1)^{T}$ and $(0,1)^{T}$ are similar. 
Claim 6.2.11. Let $M \cong M^{*}\left(K_{2 m}\right)$, then $M$ has a rank $m$ double wheel as a minor.

Proof. $M^{*} \cong M\left(K_{2 m}\right)$ and so has an $m$-rung circular ladder as a minor. Since a double wheel is the dual of a circular ladder $M$ has a rank- $m$ double wheel as a minor.

By combining the lemmas in this section, the proof of Theorem 6.2.1 becomes routine.

Theorem 6.2.1. There is a function $\sqrt{6.2 .1}$ such that the following holds. Suppose $M$ is a binary matroid with a coindependent set $X$ such that $M \backslash X \cong M^{*}\left(K_{3, n}\right)$ and $X$ is such that the following hold. Every 3-separation displayed by the canonical flower of $M \backslash X$ is blocked by an element of $X$, and the crossing graph of $X$ in $M$ is a complete graph. If $n \geq \sqrt{6.2 .1}(t)$, then $M$ has a minor isomorphic to one of the following:

1. a rank-t Möbius ladder,

2. a rank-t double wheel, or

3. $N\left(K_{3, t}\right)^{*}$.

Proof. Suppose $n \geq \int_{6.2 .4}\left(\max \left\{\int_{6.2 .6}(t), \sqrt{6.2 .7}(t) \int_{6.2 .8}(t), \sqrt{6.2 .9}(t), \sqrt{6.2 .10}(t)\right\}\right)$. By Lemma 6.2.4 $M$ has a minor that can be represented by a standard representation, $N$, of $M^{*}\left(K_{3, m^{\prime}}\right)$ augmented by a matrix $B$ with $m$ rows and of one of the following forms:

i) $B$ has $m+1$ rows the first $m$ of which form an $(\alpha, \alpha, 0)$-diagonal matrix and the last of which has all its entries equal to $\beta$ for some $\beta \in F-\{0, \alpha\}$,

ii) $B$ has $4 m$ rows the first $2 m$ of which form a $(0, \alpha, \alpha)$-diagonal matrix and the last $2 m$ of which form an $(\alpha, \alpha, 0)$-diagonal matrix,

iii) $B$ has $m$ rows and is $(\alpha, \beta, \gamma)$-diagonal with $\alpha \neq \beta, \alpha \neq 0$ and $\gamma \neq 0$,

iv) $B$ has $m+1$ rows the first of which form a $(0, \alpha, 0)$-diagonal matrix and the last of which has all entries equal to some non-zero $\beta$, 
v) $B$ is $(\alpha, \beta)$-complete for some nonzero elements $\alpha$ and $\beta$ of $F$,

where where entries of $B$ come from the set $\left\{(0,0)^{T},(0,1)^{T},(1,0)^{T},(1,1)^{T}\right\}, \alpha \neq$ 0 and $m \geq \max \left\{\int_{6.2 .6}(t), f_{6.2 .7}(t) \int_{6.2 .8}(t), f_{6.2 .9}(t), f_{6.2 .10}(t)\right\}$. By Lemma 6.2.6 if $M$ is represented by $N$ augmented by a matrix of the form described in i), then since $m \geq f 6.2 .6(t) M$ has a Möbius ladder of rank at least $t$ as a minor. The remaining cases are similar.

\subsection{Stars}

In this section we prove the following theorem.

Theorem 6.3.1. There is a function $\sqrt{6.3 .12}$ such that the following holds. Suppose $M$ is a binary matroid with a coindependent set $X$ such that $M \backslash X \cong M^{*}\left(K_{3, n}\right)$, that $X$ is such that every 3-separation displayed by the canonical flower of $M \backslash X$ is blocked by an element of $X$ and that the crossing graph of $X$ in $M$ is a star. If $n \geq 6 \overline{6.2 .1}(t)$ then $M$ has a minor isomorphic to one of the following:

i) a rank-t circular ladder,

ii) a rank-t Möbius ladder,

iii) a rank-t double wheel, or

iv) $N\left(K_{3, t}\right)^{*}$.

In this section we work under the hypotheses of Theorem 6.3.12. That is we add to our original hypotheses the following hypothesis.

- The crossing graph of $X$ with respect to $M \backslash X$ is a star.

Throughout this section we assume that $M \backslash X$ is represented by a binary matrix $N$ with respect to basis $B$.

The following lemma is Theorem 2.8 of [7].

Lemma 6.3.2. There is a function $\sqrt[6.3 .2]{ }$ with the following property. If $t$ is an integer greater than one and $A$ is an F-matrix with at least $f_{6.3 .2}(t)$ columns with no 
two columns identical and such that some column, a, crosses every other column and, for every pair of columns $b, c \in\left\{c_{1}, \ldots, c_{\sqrt[66.3 .2]{(t)}}-a\right\}, b$ and $c$ do not cross. Then A contains a row and column permuted submatrix $B$ with at least $t$ rows of one of the forms below:

$$
\left(\begin{array}{ccccc}
\beta & \alpha & \alpha & \ldots & \alpha \\
\delta & \alpha & \alpha & \ldots & \alpha \\
\delta & 0 & \alpha & \ldots & \alpha \\
\vdots & \vdots & \vdots & \ddots & \vdots \\
\delta & 0 & 0 & \ldots & \alpha \\
\gamma & 0 & 0 & \ldots & 0
\end{array}\right)
$$

(i)

$$
\left(\begin{array}{ccccc}
\delta & \alpha & 0 & \ldots & 0 \\
\delta & 0 & \alpha & \ldots & 0 \\
\vdots & \vdots & \vdots & \ddots & \vdots \\
\delta & 0 & 0 & \ldots & \alpha \\
\gamma & \beta & 0 & \ldots & 0 \\
\gamma & 0 & \beta & \ldots & 0 \\
\vdots & \vdots & \vdots & \ddots & \vdots \\
\gamma & 0 & 0 & \ldots & \beta
\end{array}\right)
$$

(ii)

where $\alpha \neq 0$ and in the first matrix $\beta \neq \delta$ and $\gamma \neq 0$ and in the final matrix $\beta, \delta \neq 0$ and $\gamma \neq \delta$.

In the case we are interested in we have $\alpha, \beta, \gamma, \delta \in$ $\left\{(1,1)^{T},(1,0)^{T},(0,1)^{T},(0,0)^{T}\right\}$ and $0=(0,0)^{T}$.

Lemma 6.3.3. There is a function 6 such that the following holds. Suppose that $\widetilde{\Lambda}$ is of the following form

$$
\left(\begin{array}{ccccc}
\beta & \alpha & \alpha & \ldots & \alpha \\
\delta & \alpha & \alpha & \ldots & \alpha \\
\delta & 0 & \alpha & \ldots & \alpha \\
\vdots & \vdots & \vdots & \ddots & \vdots \\
\delta & 0 & 0 & \ldots & \alpha \\
\gamma & 0 & 0 & \ldots & 0
\end{array}\right)
$$


where $\alpha, \gamma \neq 0$ and $\beta \neq \delta$. If $n \geq 6 \overline{6.3 .3}(t)$, then $M$ has a minor isomorphic to a rank-t circular ladder, a rank-t Möbius ladder, or a rank-t double wheel.

Proof. We shall show that $n \geq 2 m+6$ when $2 m=t$ gives the required function. Note that $r(M)=2 n-2$ Throughout this proof we may assume, without loss of generality, that $\alpha=(1,1)^{T}$. We split into cases for the possible values of $\delta$.

Claim 6.3.4. Suppose $\delta \in\left\{(1,0)^{T},(0,1)^{T}\right\}$. Then $M$ has a rank- $(2 m+2)$ circular ladder or rank $(2 m+2)$-Möbius ladder as a minor.

Proof. Without loss of generality assume that $\delta=(1,0)^{T}$. Then $\beta \in$ $\left\{(0,1)^{T},(1,1)^{T},(0,0)^{T}\right\}$. Consider $M^{\prime}=M \backslash\left\{c_{1}, \ldots, c_{n}\right\} /\left\{a_{n-1}, b_{n-1}\right\}$. This can be represented by the following matrix:

\begin{tabular}{|c|c|c|c|c|c|c|c|c|}
\hline & $a_{n}$ & $b_{n}$ & $x_{1}$ & $x_{2}$ & $x_{3}$ & $x_{4}$ & $\ldots$ & $x_{n-2}$ \\
\hline$a_{1}$ & 1 & 0 & $\beta_{1}$ & 1 & 1 & 1 & $\ldots$ & 1 \\
\hline$b_{1}$ & 0 & 1 & $\beta_{2}$ & 1 & 1 & 1 & $\ldots$ & 1 \\
\hline$a_{2}$ & 1 & 0 & 1 & 1 & 1 & 1 & $\ldots$ & 1 \\
\hline$b_{2}$ & 0 & 1 & 0 & 1 & 1 & 1 & $\ldots$ & 1 \\
\hline$a_{3}$ & 1 & 0 & 1 & 0 & 1 & 1 & $\ldots$ & 1 \\
\hline$b_{3}$ & 0 & 1 & 0 & 0 & 1 & 1 & $\ldots$ & 1 \\
\hline$a_{4}$ & 1 & 0 & 1 & 0 & 0 & 1 & $\cdot$ & 1 \\
\hline$b_{4}$ & 0 & 1 & 0 & 0 & 0 & 1 & & 1 \\
\hline$\vdots$ & $\vdots$ & $\vdots$ & $\vdots$ & $\vdots$ & $\vdots$ & $\vdots$ & $\ddots$ & $\vdots$ \\
\hline$a_{n-2}$ & 1 & 0 & 1 & 0 & 0 & 0 & $\ldots$ & 1 \\
\hline$b_{n-2}$ & 0 & 1 & 0 & 0 & 0 & 0 & $\ldots$ & 1 \\
\hline
\end{tabular}

If $\beta=(0,0)^{T}$ then $M^{\prime} \backslash a_{n} / b_{1}$ is a circular ladder of rank $2 m+2$. If $\beta=(a, 1)^{T}$ then $M^{\prime} \backslash a_{n} / a_{1}$ is a Möbius ladder of rank $2 m+2$.

Claim 6.3.5. Suppose $\delta=(0,0)^{T}$. Then $M$ has a circular ladder of rank $2 n-4$ or a Möbius ladder of rank $2 n-5$.

Proof. Suppose $\beta=(1, a)^{T}$ and $\gamma=(1, b)^{T}$ for $\{a, b\} \in\{1,0\}$. This matroid can 
be represented by:

$$
\begin{aligned}
& \begin{array}{llllllllllllll}
c_{1} & c_{2} & c_{3} & \ldots & c_{n-2} & c_{n-1} & a_{n} & b_{n} & c_{n} & x_{1} & x_{2} & x_{3} & \ldots & x_{n-2}
\end{array}
\end{aligned}
$$

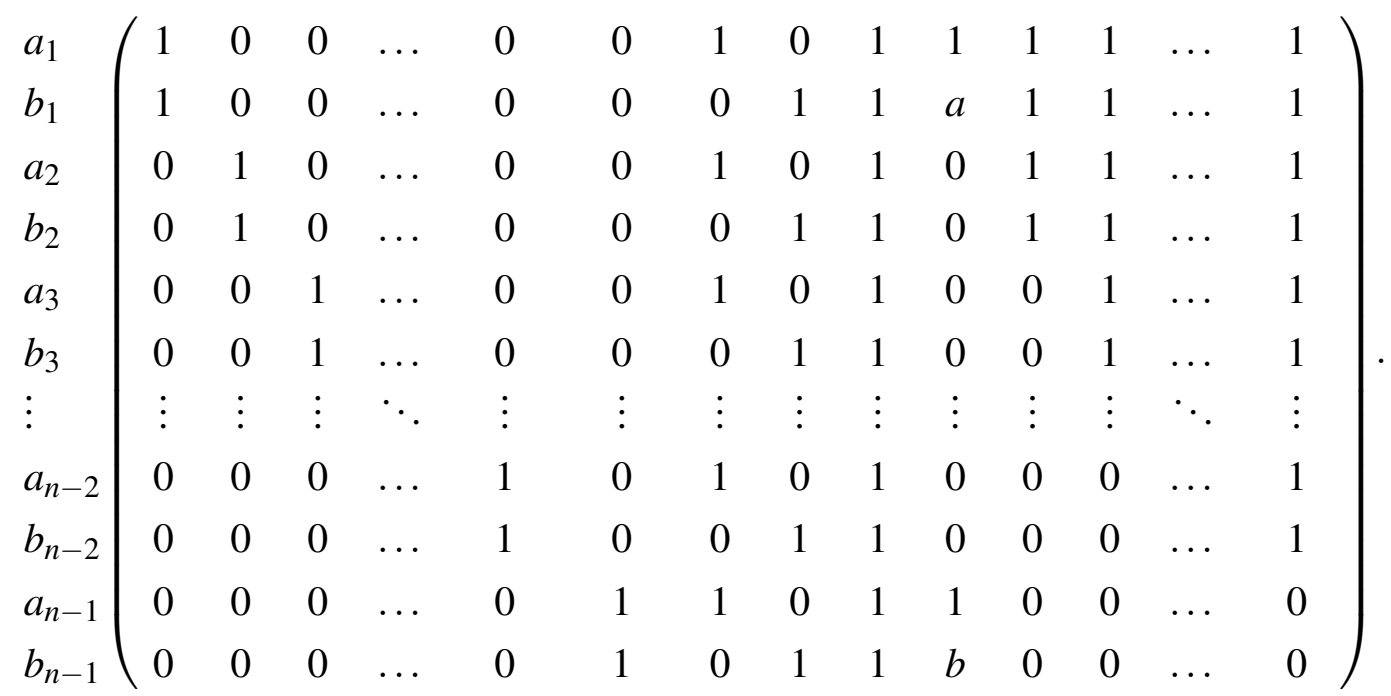

Delete $c_{1}, \ldots, c_{n-1}$, contract $b_{1}$ and $b_{n-1}$ and pivot on $M_{a_{1}, x_{1}}$ to get:

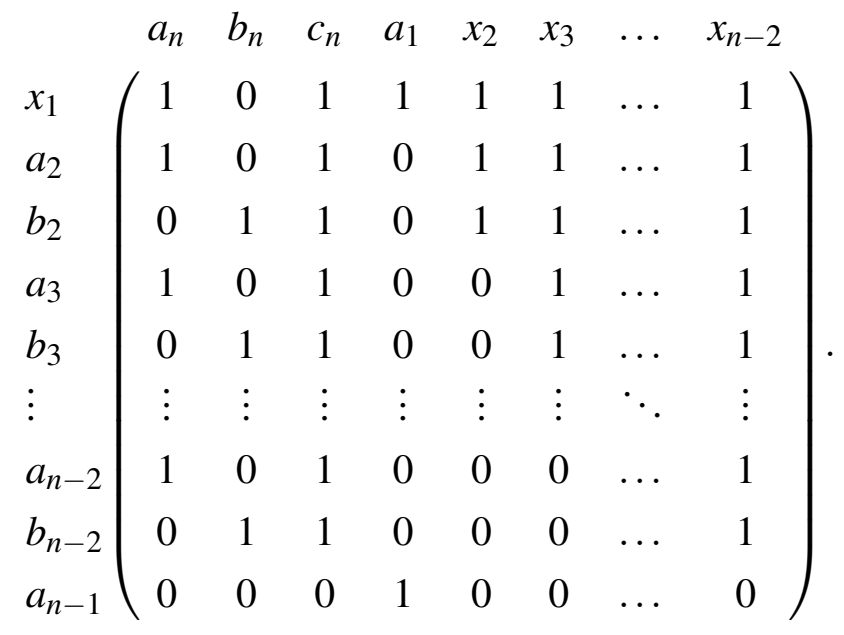

When we delete $a_{n-1}$ and $a_{1}$ this gives a circular ladder. Therefore in this case $M$ has a circular ladder of rank $2 n-2$ as a minor.

Clearly if $\beta=(a, 1)^{T}$ and $\delta=(b, 1)^{T}$ we can apply the same argument. 
Suppose $\beta=(1,0)^{T}$ and $\gamma=(0,1)^{T}$. Pivoting on $M_{a_{1}, a_{n}}$ and $M_{b_{1}, b_{n}}$ gives

$$
\left(\begin{array}{cccccccccccccc}
1 & 0 & 0 & \ldots & 0 & 0 & 1 & 0 & 1 & 1 & 1 & 1 & \ldots & 1 \\
1 & 0 & 0 & \ldots & 0 & 0 & 0 & 1 & 1 & 1 & 1 & 1 & \ldots & 1 \\
0 & 1 & 0 & \ldots & 0 & 0 & 1 & 0 & 1 & 0 & 1 & 1 & \ldots & 1 \\
0 & 1 & 0 & \ldots & 0 & 0 & 0 & 1 & 1 & 1 & 1 & 1 & \ldots & 1 \\
0 & 0 & 1 & \ldots & 0 & 0 & 1 & 0 & 1 & 0 & 0 & 1 & \ldots & 1 \\
0 & 0 & 1 & \ldots & 0 & 0 & 0 & 1 & 1 & 1 & 0 & 1 & \ldots & 1 \\
\vdots & \vdots & \vdots & \ddots & \vdots & \vdots & \vdots & \vdots & \vdots & \vdots & \vdots & \vdots & \ddots & \vdots \\
0 & 0 & 0 & \ldots & 1 & 0 & 1 & 0 & 1 & 0 & 0 & 0 & \ldots & 1 \\
0 & 0 & 0 & \ldots & 1 & 0 & 0 & 1 & 1 & 1 & 0 & 0 & \ldots & 1 \\
0 & 0 & 0 & \ldots & 0 & 1 & 1 & 0 & 1 & 0 & 1 & 0 & \ldots & 0 \\
0 & 0 & 0 & \ldots & 0 & 1 & 0 & 1 & 1 & 1 & 1 & 0 & \ldots & 0
\end{array}\right) .
$$

which is covered by the case when $\delta=(0,1)^{T}$ and $\beta=(1,1)^{T}$.

Claim 6.3.6. Suppose $\delta=(1,1)^{T}$. Then $M$ has a circular ladder of rank $2 m+2$ or a Möbius ladder of rank $2 m+1$ as a minor.

Proof. When $\delta=(1,1)^{T}$ we have the following matrix:

$$
\begin{aligned}
& \begin{array}{llllllllllllll}
c_{1} & c_{2} & c_{3} & \ldots & c_{n-2} & c_{n-1} & a_{n} & b_{n} & c_{n} & x_{1} & x_{2} & x_{3} & \ldots & x_{n-2}
\end{array}
\end{aligned}
$$

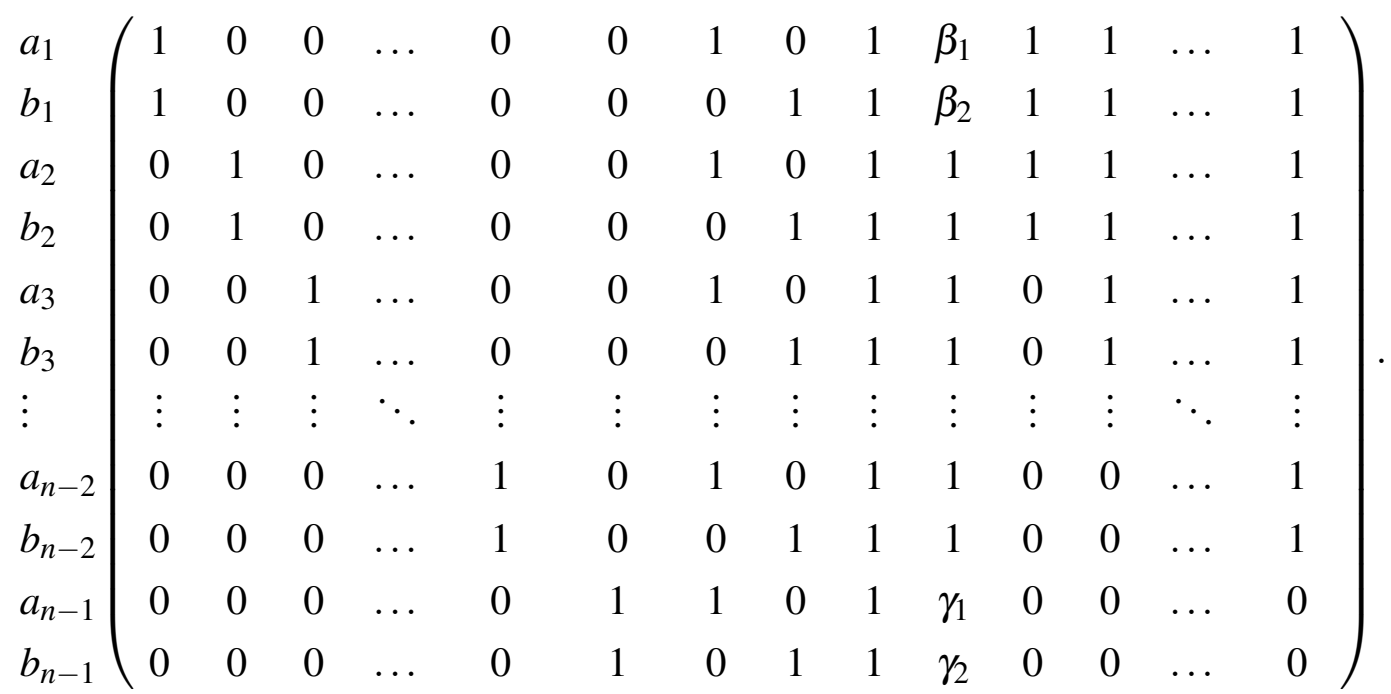


Pivot on $M_{a_{1}, a_{n}}$ and $M_{b_{1}, b_{n}}$ to get

$$
\left(\begin{array}{cccccccccccccc}
1 & 0 & 0 & \ldots & 0 & 0 & 1 & 0 & 1 & \beta_{1}+\gamma_{1} & 1 & 1 & \ldots & 1 \\
1 & 0 & 0 & \ldots & 0 & 0 & 0 & 1 & 1 & \beta_{2}+\gamma_{2} & 1 & 1 & \ldots & 1 \\
0 & 1 & 0 & \ldots & 0 & 0 & 1 & 0 & 1 & 1+\gamma_{1} & 1 & 1 & \ldots & 1 \\
0 & 1 & 0 & \ldots & 0 & 0 & 0 & 1 & 1 & 1+\gamma_{2} & 1 & 1 & \ldots & 1 \\
0 & 0 & 1 & \ldots & 0 & 0 & 1 & 0 & 1 & 1+\gamma_{1} & 0 & 1 & \ldots & 1 \\
0 & 0 & 1 & \ldots & 0 & 0 & 0 & 1 & 1 & 1+\gamma_{2} & 0 & 1 & \ldots & 1 \\
\vdots & \vdots & \vdots & \ddots & \vdots & \vdots & \vdots & \vdots & \vdots & \vdots & \vdots & \vdots & \ddots & \vdots \\
0 & 0 & 0 & \ldots & 1 & 0 & 1 & 0 & 1 & 1+\gamma_{1} & 0 & 0 & \ldots & 1 \\
0 & 0 & 0 & \ldots & 1 & 0 & 0 & 1 & 1 & 1+\gamma_{2} & 0 & 0 & \ldots & 1 \\
0 & 0 & 0 & \ldots & 0 & 1 & 1 & 0 & 1 & \gamma_{1} & 0 & 0 & \ldots & 0 \\
0 & 0 & 0 & \ldots & 0 & 1 & 0 & 1 & 1 & \gamma_{2} & 0 & 0 & \ldots & 0
\end{array}\right) .
$$

We are now in the case where $\delta \in\left\{(0,0)^{T},(0,1)^{T},(1,0)^{T}\right\}$ and the result follows.

Putting theses claims together we see that if $n \geq t+6$, then then $M$ has a rank- $t$ circular ladder or a rank- $t$ Möbius ladder as a minor.

When $\widetilde{\Lambda}$ is of the form $i$ ) from Lemma 6.3.2 we shall see that finding the unavoidable minors of $M$ reduces to the dual of one of the cases we have already seen for blocking $M\left(K_{3, n}\right)$ as a minor, although the case analysis is painful!

Lemma 6.3.7. Suppose $M$ is represented by

$$
\left(\begin{array}{ccccccccccccccc}
1 & 1 & 0 & 0 & 0 & 0 & \ldots & 0 & 0 & 1 & 1 & 1 & 1 & \ldots & 1 \\
0 & 0 & 1 & 1 & 0 & 0 & \ldots & 0 & 0 & 1 & 0 & 0 & 0 & \ldots & 0 \\
0 & 0 & 0 & 0 & 1 & 1 & \ldots & 0 & 0 & 0 & 1 & 0 & 0 & \ldots & 0 \\
\vdots & \vdots & \vdots & \vdots & \vdots & \vdots & \ddots & \vdots & \vdots & \vdots & \vdots & \vdots & \vdots & \ddots & \vdots \\
0 & 0 & 0 & 0 & \ldots & 1 & 1 & 0 & 0 & 0 & 0 & 0 & 0 & \ldots & 1 \\
1 & 0 & 1 & 0 & \ldots & 1 & 0 & 1 & 1 & ? & ? & ? & ? & \ldots & ? \\
1 & 1 & 1 & 1 & \ldots & 1 & 1 & 1 & 1 & ? & ? & ? & ? & \ldots & ?
\end{array}\right)^{T}
$$

If $n \geq \int 5.2 .9(t)$ then $M$ has a minor isomorphic to $M^{*}\left(K_{4, t}\right)$.

Proof. This follows from the dual of Lemma 5.2.9. 
Lemma 6.3.8. There is a function $\sqrt{6.3 .8}$ such that the following holds. Suppose $\widetilde{\Lambda}$ is of form

$$
\left(\begin{array}{ccccc}
\delta & \alpha & 0 & \ldots & 0 \\
\delta & 0 & \alpha & \ldots & 0 \\
\vdots & \vdots & \vdots & \ddots & \vdots \\
\delta & 0 & 0 & \ldots & \alpha \\
\gamma & \beta & 0 & \ldots & 0 \\
\gamma & 0 & \beta & \ldots & 0 \\
\vdots & \vdots & \vdots & \ddots & \vdots \\
\gamma & 0 & 0 & \ldots & \beta
\end{array}\right)
$$

where $\alpha, \delta, \beta \neq 0$ and $\gamma \neq \delta$. If $n \geq 6$ 6.3.8 $(t)$ then $M$ has a rank-t minor isomorphic to $M^{*}\left(K_{4, t}\right)$.

Proof. Let $n=2 m+5$ where $m=\frac{1}{3} \sqrt{5.2 .9}(t)$ and so the rank of the $M$ is $4 m+8$. We start by showing that $M$ has a minor with reduced standard representation

$$
\left(\begin{array}{ccccccccccccccc}
1 & 1 & 0 & 0 & 0 & 0 & \ldots & 0 & 0 & 1 & 1 & 1 & 1 & \ldots & 1 \\
0 & 0 & 1 & 1 & 0 & 0 & \ldots & 0 & 0 & 1 & 0 & 0 & 0 & \ldots & 0 \\
0 & 0 & 0 & 0 & 1 & 1 & \ldots & 0 & 0 & 0 & 1 & 0 & 0 & \ldots & 0 \\
\vdots & \vdots & \vdots & \vdots & \vdots & \vdots & \ddots & \vdots & \vdots & \vdots & \vdots & \vdots & \vdots & \ddots & \vdots \\
0 & 0 & 0 & 0 & \ldots & 1 & 1 & 0 & 0 & 0 & 0 & 0 & 0 & \ldots & 1 \\
1 & 0 & 1 & 0 & \ldots & 1 & 0 & 1 & 1 & ? & ? & ? & ? & \ldots & ? \\
1 & 1 & 1 & 1 & \ldots & 1 & 1 & 1 & 1 & ? & ? & ? & ? & \ldots & ?
\end{array}\right)^{T}
$$

Claim 6.3.9. There is a minor $M^{\prime}$ of $M$ such that $M^{\prime}$ can be represented by a standard representation of $M^{*}\left(K_{3,2 m+3}\right)$ augmented by a matrix of the form given in $i i)$ of Lemma 6.3.2 with $\alpha=(1,1)$ and $\gamma=(0,0)$.

Proof. Pivot on $M_{a_{n-1}, a_{n}}$ and $M_{b_{n-1}, b_{n}}$ to obtain a standard representation of 
$M^{*}\left(K_{3, n}\right)$ augmented by a matrix of the form below.

$$
\left(\begin{array}{cccccc}
\delta_{1}+\gamma_{1} & \alpha_{1} & 0 & \ldots & 0 & \beta_{1} \\
\delta_{2}+\gamma_{2} & \alpha_{2} & 0 & \ldots & 0 & \beta_{2} \\
\delta_{1}+\gamma_{1} & 0 & \alpha_{1} & \ldots & 0 & \beta_{1} \\
\delta_{2}+\gamma_{2} & 0 & \alpha_{2} & \ldots & 0 & \beta_{2} \\
\vdots & \vdots & \vdots & \ddots & \vdots & \vdots \\
\delta_{1}+\gamma_{1} & 0 & 0 & \ldots & \alpha_{1} & \beta_{1} \\
\delta_{2}+\gamma_{2} & 0 & 0 & \ldots & \alpha_{2} & \beta_{2} \\
\delta_{1}+\gamma_{1} & 0 & 0 & \ldots & 0 & \alpha_{1}+\beta_{1} \\
\delta_{2}+\gamma_{2} & 0 & 0 & \ldots & 0 & \alpha_{2}+\beta_{2} \\
\gamma_{1}+\gamma_{1} & \beta_{1} & 0 & \ldots & 0 & \beta_{1} \\
\gamma_{2}+\gamma_{2} & \beta_{2} & 0 & \ldots & 0 & \beta_{2} \\
\gamma_{1}+\gamma_{1} & 0 & \beta_{1} & \ldots & 0 & \beta_{1} \\
\gamma_{2}+\gamma_{2} & 0 & \beta_{2} & \ldots & 0 & \beta_{2} \\
\vdots & \vdots & \vdots & \ddots & \vdots & \vdots \\
\gamma_{1}+\gamma_{1} & 0 & 0 & \ldots & \beta_{1} & \beta_{1} \\
\gamma_{2}+\gamma_{2} & 0 & 0 & \ldots & \beta_{2} & \beta_{2} \\
\gamma_{1} & 0 & 0 & \ldots & 0 & \beta_{1} \\
\gamma_{2} & 0 & 0 & \ldots & 0 & \beta_{2}
\end{array}\right)
$$

Since the matroid is binary $\gamma_{i}+\gamma_{i}=0$ for $i \in\{1,0\}$. For this to be of form $\left.i i\right)$ we must have $\delta_{i}+\gamma_{i} \neq 0$ for $i \in\{0,1\}$. Since $\delta \neq(0,0)^{T}$ and $\delta \neq \gamma$ this follows. Since $n=2 m+5$, it is now clear that we can obtain the required minor.

For the remainder of the proof let $M^{\prime}$ be a minor of $M$ such that $M^{\prime}$ can be represented by a standard representation of $M^{*}\left(K_{3,2 m+3}\right)$ augmented by a matrix of the form given in $i i)$ of Lemma 6.3.2 with $\alpha=(1,1)$ and $\gamma=(0,0)$.

Claim 6.3.10. When $\delta=(1,1), M^{\prime}$ has a minor of rank at least $3 m$ with 
reduced standard representation

$$
\left(\begin{array}{ccccccccccccccc}
1 & 1 & 0 & 0 & 0 & 0 & \ldots & 0 & 0 & 1 & 1 & 1 & 1 & \ldots & 1 \\
0 & 0 & 1 & 1 & 0 & 0 & \ldots & 0 & 0 & 1 & 0 & 0 & 0 & \ldots & 0 \\
0 & 0 & 0 & 0 & 1 & 1 & \ldots & 0 & 0 & 0 & 1 & 0 & 0 & \ldots & 0 \\
\vdots & \vdots & \vdots & \vdots & \vdots & \vdots & \ddots & \vdots & \vdots & \vdots & \vdots & \vdots & \vdots & \ddots & \vdots \\
0 & 0 & 0 & 0 & \ldots & 1 & 1 & 0 & 0 & 0 & 0 & 0 & 0 & \ldots & 1 \\
1 & 0 & 1 & 0 & \ldots & 1 & 0 & 1 & 1 & ? & ? & ? & ? & \ldots & ? \\
1 & 1 & 1 & 1 & \ldots & 1 & 1 & 1 & 1 & ? & ? & ? & ? & \ldots & ?
\end{array}\right)^{T}
$$

Proof. Without loss of generality let $\beta=(1, a)^{T}$ for $a \in\{0,1\}$. Consider $M \backslash$ $\left\{c_{1}, \ldots, c_{2 m+4}\right\}$. This is represented by the matrix below:

\begin{tabular}{|c|c|c|c|c|c|c|c|c|}
\hline & $a_{2 m+5}$ & $b_{2 m+5}$ & $c_{2 m+5}$ & $x_{1}$ & $x_{2}$ & $x_{3}$ & $\ldots$ & $x_{m+3}$ \\
\hline$a_{1}$ & 1 & 0 & 1 & 1 & 1 & 0 & $\ldots$ & 0 \\
\hline$b_{1}$ & 0 & 1 & 1 & 1 & 1 & 0 & $\ldots$ & 0 \\
\hline$a_{2}$ & 1 & 0 & 1 & 1 & 0 & 1 & $\ldots$ & 0 \\
\hline$b_{2}$ & 0 & 1 & 1 & 1 & 0 & 1 & $\ldots$ & 0 \\
\hline$\vdots$ & $\vdots$ & $\vdots$ & $\vdots$ & $\vdots$ & $\vdots$ & $\vdots$ & $\ddots$. & $\vdots$ \\
\hline$a_{m+2}$ & 1 & 0 & 1 & 1 & 0 & 0 & $\ldots$ & 1 \\
\hline$b_{m+2}$ & 0 & 1 & 1 & 1 & 0 & 0 & $\ldots$ & 1 \\
\hline$a_{m+3}$ & 1 & 0 & 1 & 0 & 1 & 0 & $\ldots$ & 0 \\
\hline$b_{m+3}$ & 0 & 1 & 1 & 0 & $a$ & 0 & $\ldots$ & 0 \\
\hline$a_{m+4}$ & 1 & 0 & 1 & 0 & 0 & 1 & $\ldots$ & 0 \\
\hline$b_{m+4}$ & 0 & 1 & 1 & 0 & 0 & $a$ & $\ldots$ & 0 \\
\hline$\vdots$ & $\vdots$ & $\vdots$ & $\vdots$ & $\vdots$ & $\vdots$ & $\vdots$ & $\ddots$ & $\vdots$ \\
\hline$a_{2 m+4}$ & 1 & 0 & 1 & 0 & 0 & 0 & $\ldots$ & 1 \\
\hline$b_{2 m+4}$ & 0 & 1 & 1 & 0 & 0 & 0 & $\ldots$ & $a$ \\
\hline
\end{tabular}


Pivot on $M_{a_{1}, c_{2 m+5}}$ to get:

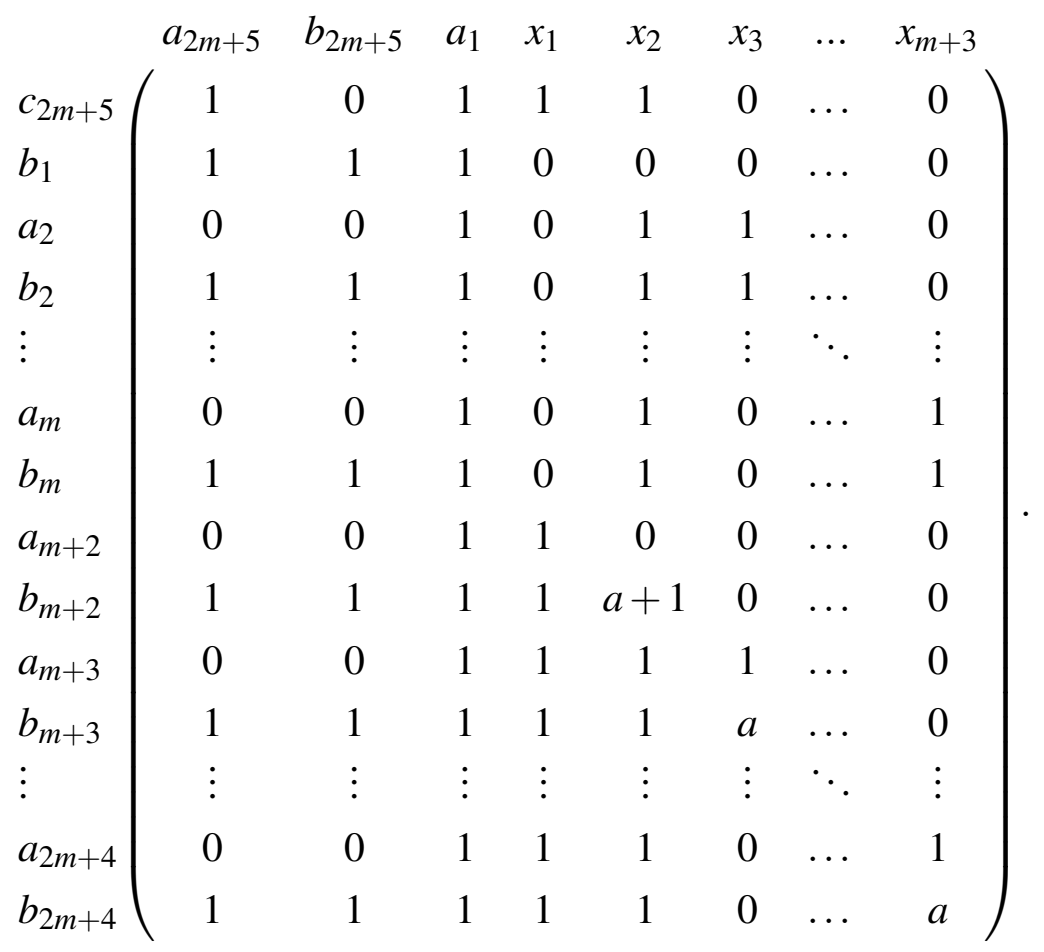

Contract $b_{m+2}, \ldots, b_{2 m+2}, b_{1}, c_{2 m+3}$ and delete $a_{2 m+3}$ and $x_{2}$. After rearranging rows we get

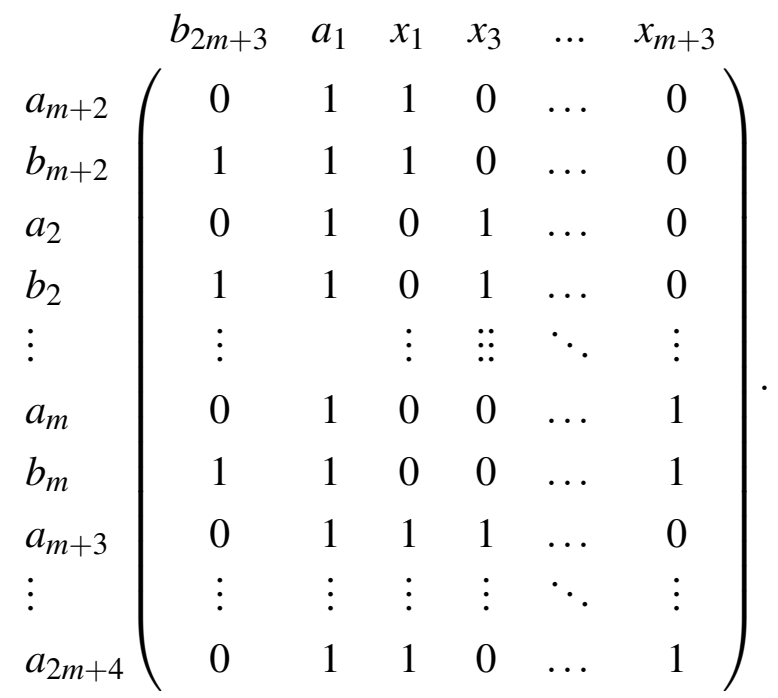

This is a rank $2 m+1$ matrix of the required form.

Claim 6.3.11. When $\delta=(1,0)$ and $\gamma=(0,0), M$ has a minor of rank at 
least $3 m$ represented by

$$
\left(\begin{array}{ccccccccccccccc}
1 & 1 & 0 & 0 & 0 & 0 & \ldots & 0 & 0 & 1 & 1 & 1 & 1 & \ldots & 1 \\
0 & 0 & 1 & 1 & 0 & 0 & \ldots & 0 & 0 & 1 & 0 & 0 & 0 & \ldots & 0 \\
0 & 0 & 0 & 0 & 1 & 1 & \ldots & 0 & 0 & 0 & 1 & 0 & 0 & \ldots & 0 \\
\vdots & \vdots & \vdots & \vdots & \vdots & \vdots & \ddots & \vdots & \vdots & \vdots & \vdots & \vdots & \vdots & \ddots & \vdots \\
0 & 0 & 0 & 0 & 0 & 0 & \ldots & 1 & 1 & 0 & 0 & 0 & 0 & \ldots & 1 \\
1 & 0 & 1 & 0 & 1 & 0 & \ldots & 1 & 0 & 1 & 1 & ? & ? & ? \ldots & ? \\
1 & 1 & 1 & 1 & 1 & 1 & \ldots & 1 & 1 & 1 & 1 & ? & ? & \ldots & ?
\end{array}\right)^{T}
$$

Proof. First suppose that $\beta=(1, a)$. Delete $c_{1}, \ldots, c_{2 m+2}$ and contract $b_{m+1}, \ldots, b_{2 m+2}$ to get:

\begin{tabular}{|c|c|c|c|c|c|c|c|c|c|}
\hline & $a_{2 m+5}$ & $b_{2 m+5}$ & $c_{2 m+5}$ & $x_{1}$ & $x_{2}$ & $x_{3}$ & $x_{4}$ & $\ldots$ & $x_{m+3}$ \\
\hline$a_{1}$ & 1 & 0 & 1 & 1 & 1 & 0 & 0 & $\ldots$ & 0 \\
\hline$b_{1}$ & 0 & 1 & 1 & 0 & 1 & 0 & 0 & $\ldots$ & 0 \\
\hline$a_{2}$ & 1 & 0 & 1 & 1 & 0 & 1 & 0 & $\ldots$ & 0 \\
\hline$b_{2}$ & 0 & 1 & 1 & 0 & 0 & 1 & 0 & $\ldots$ & 0 \\
\hline$a_{3}$ & 1 & 0 & 1 & 1 & 0 & 0 & 0 & $\ldots$ & 0 \\
\hline$b_{3}$ & 0 & 1 & 1 & 0 & 0 & 0 & 0 & $\ldots$ & 0 \\
\hline$\vdots$ & $\vdots$ & $\vdots$ & $\vdots$ & $\vdots$ & $\vdots$ & $\vdots$ & $\vdots$ & $\ddots$ & $\vdots$ \\
\hline$a_{m+2}$ & 1 & 0 & 1 & 1 & 0 & 0 & 0 & $\ldots$ & 1 \\
\hline$b_{m+2}$ & 0 & 1 & 1 & 0 & 0 & 0 & 0 & $\ldots$ & 1 \\
\hline$a_{m+3}$ & 1 & 0 & 1 & 0 & 1 & 0 & 0 & $\ldots$ & 0 \\
\hline$a_{m+3}$ & 1 & 0 & 1 & 0 & 0 & 1 & 0 & $\ldots$ & 0 \\
\hline$a_{m+4}$ & 1 & 0 & 1 & 0 & 0 & 0 & 1 & $\ldots$ & 0 \\
\hline$\vdots$ & $\vdots$ & $\vdots$ & $\ddots$ & $\vdots$ & & & & & \\
\hline$a_{2 m+4}$ & 1 & 0 & 1 & 0 & 0 & 0 & 0 & $\ldots$ & 1 \\
\hline
\end{tabular}


Pivot on $M_{a_{1}, x_{1}}$ to give the matrix below

\begin{tabular}{|c|c|c|c|c|c|c|c|c|c|}
\hline & $a_{2 m+5}$ & $b_{2 m+5}$ & $c_{2 m+5}$ & $a_{1}$ & $x_{2}$ & $x_{3}$ & $x_{4}$ & $\ldots$ & $x_{m+3}$ \\
\hline$x_{1}$ & 1 & 0 & 1 & 1 & 1 & 0 & 0 & $\ldots$ & 0 \\
\hline$b_{1}$ & 0 & 1 & 1 & 0 & 1 & 0 & 0 & $\ldots$ & 0 \\
\hline$a_{2}$ & 0 & 0 & 0 & 1 & 1 & 1 & 0 & $\ldots$ & 0 \\
\hline$b_{2}$ & 0 & 1 & 1 & 0 & 0 & 1 & 0 & $\ldots$ & 0 \\
\hline$a_{3}$ & 0 & 0 & 0 & 1 & 1 & 0 & 1 & $\ldots$ & 0 \\
\hline$b_{3}$ & 0 & 1 & 1 & 0 & 0 & 0 & 1 & $\ldots$ & 0 \\
\hline$\vdots$ & $\vdots$ & $\vdots$ & $\vdots$ & $\vdots$ & $\vdots$ & $\vdots$ & $\vdots$ & $\ddots$ & $\vdots$ \\
\hline$a_{m+2}$ & 0 & 0 & 0 & 1 & 1 & 0 & 0 & $\ldots$ & 1 \\
\hline$b_{m+2}$ & 0 & 1 & 1 & 0 & 0 & 0 & 0 & $\ldots$ & 1 \\
\hline$a_{m+3}$ & 1 & 0 & 1 & 0 & 1 & 0 & 0 & $\ldots$ & 0 \\
\hline$a_{m+4}$ & 1 & 0 & 1 & 0 & 0 & 1 & 0 & $\ldots$ & 0 \\
\hline$a_{m+5}$ & 1 & 0 & 1 & 0 & 0 & 0 & 1 & $\ldots$ & 0 \\
\hline$\vdots$ & $\vdots$ & $\vdots$ & $\ddots$ & $\vdots$ & & & & & \\
\hline$a_{2 m+4}$ & 1 & 0 & 1 & 0 & 0 & 0 & 0 & & 1 \\
\hline
\end{tabular}

$M \backslash\left\{c_{2 m+5}, a_{1}\right\} / x_{1}$ is represented by

\begin{tabular}{|c|c|c|c|c|c|c|c|}
\hline & $a_{2 m+5}$ & $b_{2 m+5}$ & $x_{2}$ & $x_{3}$ & $x_{4}$ & $\ldots$ & $x_{m+3}$ \\
\hline$a_{m+3}$ & 1 & 0 & 1 & 0 & 0 & $\ldots$ & 0 \\
\hline$b_{1}$ & 0 & 1 & 1 & 0 & 0 & $\ldots$ & 0 \\
\hline$a_{m+4}$ & 1 & 0 & 0 & 1 & 0 & $\ldots$ & 0 \\
\hline$b_{2}$ & 0 & 1 & 0 & 1 & 0 & $\ldots$ & 0 \\
\hline$a_{m+5}$ & 1 & 0 & 0 & 0 & 1 & $\ldots$ & 0 \\
\hline$b_{3}$ & 0 & 1 & 0 & 0 & 1 & $\ldots$ & 0 \\
\hline$\vdots$ & $\vdots$ & $\vdots$ & $\vdots$ & $\vdots$ & $\vdots$ & $\ddots$ & $\vdots$ \\
\hline$a_{2 m+3}$ & 1 & 0 & 0 & 0 & 0 & $\ldots$ & 1 \\
\hline$b_{m+3}$ & 0 & 1 & 0 & 0 & 0 & $\ldots$ & 1 \\
\hline$a_{2}$ & 0 & 0 & 1 & 1 & 0 & $\ldots$ & 0 \\
\hline$a_{3}$ & 0 & 0 & 1 & 0 & 1 & $\ldots$ & 0 \\
\hline$\vdots$ & $\vdots$ & $\vdots$ & $\vdots$ & $\vdots$ & $\vdots$ & $\ddots$ & $\vdots$ \\
\hline$a_{2 m+4}$ & 0 & 0 & 1 & 0 & 0 & $\ldots$ & 1 \\
\hline
\end{tabular}

Contracting $a_{2 m+4}$ and $b_{m+3}$ gives the required matrix. 
Suppose $\beta=(0,1)$. Pivot on $M_{b_{i}, c_{i}}$ for $i \in\{m+1, \ldots, 2 m+4\}$ and delete $c_{1}, \ldots, c_{2 m+4}, b_{m+3}, \ldots, b_{2 m+4}$ to get

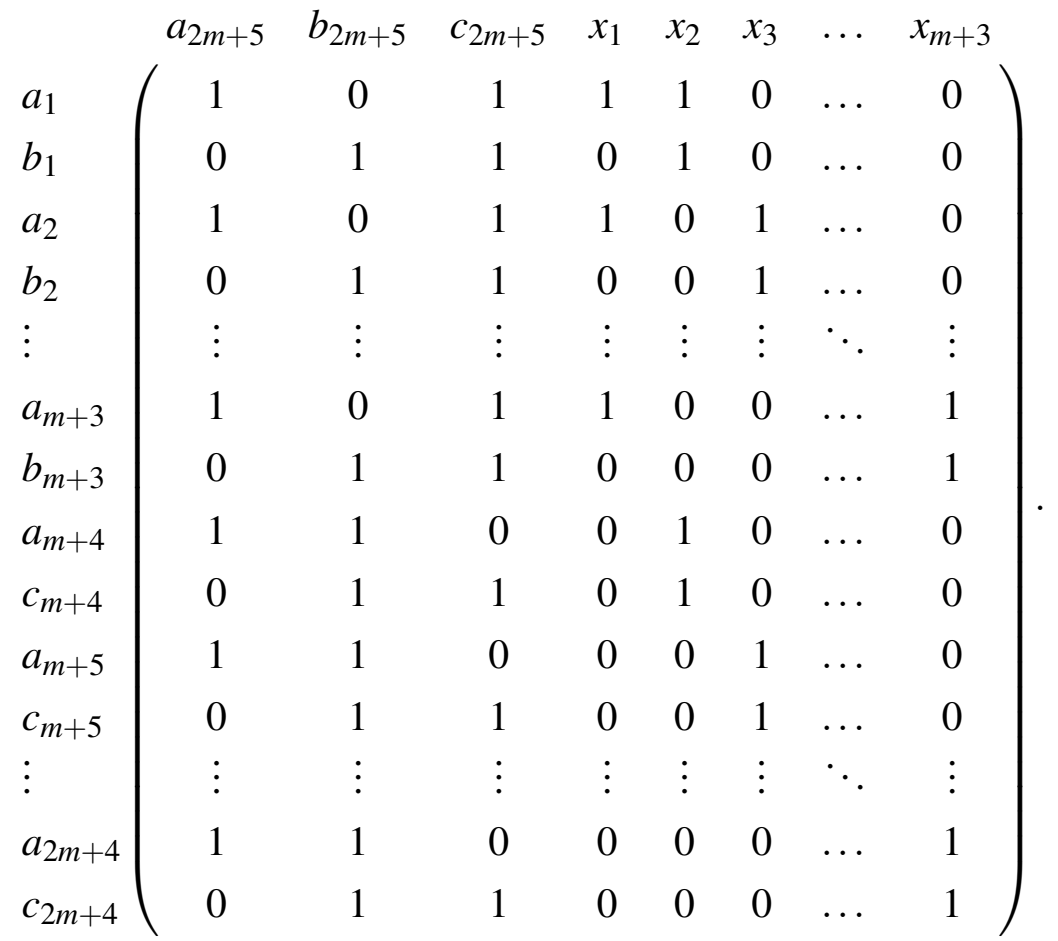

Pivoting on $M_{a_{1}, x_{1}}$ the gives

\begin{tabular}{|c|c|c|c|c|c|c|c|c|}
\hline & $a_{2 m+5}$ & $b_{2 m+5}$ & $c_{2 m+5}$ & $a_{1}$ & $x_{2}$ & $x_{3}$ & $\ldots$ & $x_{m+3}$ \\
\hline$x_{1}$ & 1 & 0 & 1 & 1 & 1 & 0 & $\ldots$ & 0 \\
\hline$b_{1}$ & 0 & 1 & 1 & 0 & 1 & 0 & $\ldots$ & 0 \\
\hline$a_{2}$ & 0 & 0 & 0 & 1 & 1 & 1 & $\ldots$ & 0 \\
\hline$b_{2}$ & 0 & 1 & 1 & 0 & 0 & 1 & $\ldots$ & 0 \\
\hline$\vdots$ & $\vdots$ & $\vdots$ & $\vdots$ & $\vdots$ & $\vdots$ & $\vdots$ & $\ddots$ & $\vdots$ \\
\hline$a_{m+2}$ & 0 & 0 & 0 & 1 & 1 & 0 & $\ldots$ & 1 \\
\hline$b_{m+2}$ & 0 & 1 & 1 & 0 & 0 & 0 & $\ldots$ & 1 \\
\hline$a_{m+3}$ & 1 & 1 & 0 & 0 & 1 & 0 & $\ldots$ & 0 \\
\hline$c_{m+3}$ & 0 & 1 & 1 & 0 & 1 & 0 & $\ldots$ & 0 \\
\hline$a_{m+4}$ & 1 & 1 & 0 & 0 & 0 & 1 & $\ldots$ & 0 \\
\hline$c_{m+4}$ & 0 & 1 & 1 & 0 & 0 & 1 & $\ldots$ & 0 \\
\hline$\vdots$ & $\vdots$ & $\vdots$ & $\vdots$ & $\vdots$ & $\vdots$ & $\vdots$ & $\ddots$ & $\vdots$ \\
\hline$a_{2 m+4}$ & 1 & 1 & 0 & 0 & 0 & 0 & $\ldots$ & 1 \\
\hline$c_{2 m+4}$ & 0 & 1 & 1 & 0 & 0 & 0 & $\ldots$ & 1 \\
\hline
\end{tabular}


Deleting $a_{2 m+5}$ and $a_{1}$ and contracting $x_{1}, b_{1}, b_{2}, \ldots, b_{m+2}$ gives the required matrix.

Combining these claims we find that $M$ must have a rank $3 m$ minor of form

$$
\left(\begin{array}{ccccccccccccccc}
1 & 1 & 0 & 0 & 0 & 0 & \ldots & 0 & 0 & 1 & 1 & 1 & 1 & \ldots & 1 \\
0 & 0 & 1 & 1 & 0 & 0 & \ldots & 0 & 0 & 1 & 0 & 0 & 0 & \ldots & 0 \\
0 & 0 & 0 & 0 & 1 & 1 & \ldots & 0 & 0 & 0 & 1 & 0 & 0 & \ldots & 0 \\
\vdots & \vdots & \vdots & \vdots & \vdots & \vdots & \ddots & \vdots & \vdots & \vdots & \vdots & \vdots & \vdots & \ddots & \vdots \\
0 & 0 & 0 & 0 & \ldots & 1 & 1 & 0 & 0 & 0 & 0 & 0 & 0 & \ldots & 1 \\
1 & 0 & 1 & 0 & \ldots & 1 & 0 & 1 & 1 & ? & ? & ? & ? & \ldots & ? \\
1 & 1 & 1 & 1 & \ldots & 1 & 1 & 1 & 1 & ? & ? & ? & ? & \ldots & ?
\end{array}\right)^{T}
$$

Since $m=\frac{1}{3} \int_{5.2 .9}(t)$ it follows from Lemma 6.3 .7 that $M$ has a minor isomorphic to $M^{*}\left(K_{4, t}\right)$.

The proof of Theorem 6.3.12 is now routine.

Theorem 6.3.12. There is a function $\sqrt{6.3 .12}$ such that the following holds. Suppose $M$ is a binary matroid with a coindependent set $X$ such that $M \backslash X \cong M^{*}\left(K_{3, n}\right)$, that $X$ is such that every 3-separation displayed by the canonical flower of $M \backslash X$ is blocked by an element of $X$ and that the crossing graph of $X$ in $M$ is a star. If $n \geq \sqrt[6.2 .1]{ }(t)$ then $M$ has a minor isomorphic to one of the following:

i) a rank-t circular ladder,

ii) a rank-t Möbius ladder,

iii) a rank-t double wheel, or

iv) $N\left(K_{3, t}\right)^{*}$.

Proof. Let $\sqrt{6.3 .12}=\int_{6.3 .2}\left(\max \left\{f_{6.3 .3}, 6[6.2 .9\}\right)\right.$ Since $\int_{6.3 .12}(t)=\int_{6.3 .2}(m)$ where $m=\max \left\{f_{6.3 .3}(t), f_{6.2 .9}(t)\right\}$, it follows easily from Lemma 6.3.2 that $M$ has a minor, $M^{\prime}$, that can be represented by a reduced standard representation of $K_{3, m}$ augmented by a matrix of for $i$ ) or $i i)$ from Lemma 6.3.2. Since $m \geq \sqrt{6.3 .3}(t)$, if $M^{\prime}$ can be represented by a reduced standard representation of $M\left(K_{3, m}\right)$ augmented by a matrix of for $i$ ), then, by Lemma 6.3.3, $M^{\prime}$ has a minor isomorphic to a 
rank- $t$ circular ladder, a rank- $t$ Möbius ladder or a rank- $t$ double wheel. Since $m \geq \sqrt[6.2 .9]{ }(t)$, if $M^{\prime}$ can be represented by a reduced standard representation of $M\left(K_{3, m}\right)$ augmented by a matrix of for $\left.i i\right)$, then, by Lemma 6.2.9, $M^{\prime}$ has a minor isomorphic $M^{*}\left(K_{4, t}\right)$.

\subsection{Paths}

In the previous version of this thesis we believed we had a way to reduce this case to a spike. However, this turned out to be incorrect and the analysis of this case will be done at a later stage.

\subsection{Proof of Theorem 6.0.1}

We now have all the results we need for a routine proof of Theorem 6.0.1

Theorem 6.0.1. There is a function $\sqrt{6.0 .1}$ such that the following hold. Suppose $M$ is a binary matroid and $X$ a coindependent set in $M$ such that $M \backslash X \cong M^{*}\left(K_{3, n}\right)$ where $n \geq 6$ 6.0.1 $(t)$. If $M$ is not blocked in a path-like way and every 3-separation of $M \backslash X$ displayed by the canonical flower of $M \backslash X$ is blocked by some element $x \in X$, then M has a minor isomorphic to one of the following matroids.
i) A rank-t circular ladder,
ii) a rank-t Möbius ladder,
iii) a rank-t double wheel,
iv) $\left(N\left(K_{3, t}^{*}\right)\right)^{*}$.

Proof. Suppose $n \geq \sqrt{6.1 .9}\left(\max \left\{f_{6.2 .1}(t), f_{6.3 .12}(t), f_{? ?}(t)\right)\right\}$. By Theorem 6.1.9 $M$ has a minor $M^{\prime}$ with coindependent set $X^{\prime}=X \cap E\left(M^{\prime}\right)$ such that $M^{\prime} \backslash X^{\prime} \cong$ $M^{*}\left(K_{3, n}\right)$, every 3-separation of $M^{\prime} \backslash X^{\prime}$ displayed by the canonical flower of $M^{\prime} \backslash$ $X^{\prime}$ is blocked by an element of $X^{\prime}$, the crossing graph on the elements of $X$ in $M^{\prime}$ is either a star, a path, or a complete graph and $M^{\prime} \backslash X^{\prime} \cong K_{3, m^{\prime}}$ where $m^{\prime}=$ $\left.\max \left\{6 \frac{6.2 .1}{6 t}(t), f_{6.3 .12}(t), f_{? ? ?}(t)\right)\right\}$. 
If the crossing graph of $X^{\prime}$ in $M^{\prime}$ is a complete graph then, since $m^{\prime} \geq \sqrt{6.2 .1}(t)$, by Theorem 6.2.1 $M^{\prime}$ and hence $M$ has a rank- $t$ double wheel, a rank- $t$ Möbius ladder or a $N\left(K_{3, t}\right)^{*}$-minor.

If the crossing graph of $X^{\prime}$ in $M^{\prime}$ is a star, then since $m^{\prime} \geq 6$ 6.3.12 $(t)$, by Theorem 6.3.12 $M^{\prime}$ and hence $M$ has a rank- $t$ double wheel, a rank- $t$ Möbius ladder, or a rank- $t$ circular ladder minor. 


\section{Chapter 7}

\section{Bridging $M\left(K_{3, n}\right)$ and $M^{*}\left(K_{3, n}\right)$}

In this chapter we give the unavoidable minors of a 4-connected matroid with an $M\left(K_{3, n}\right)$ or $M^{*}\left(K_{3, n}\right)$-minor and no large spike minor. Throughout this chapter we work under the following hypotheses.

- $M$ is a 4-connected binary matroid of rank $n$ for some large $n$.

- For some independent set $C \subseteq E(M)$ and some coindependent set $D \subseteq E(M)$ the matroid $M / C \backslash D \cong M\left(K_{3, n}\right)$.

In this section we reduce the problem of bridging $M\left(K_{3, n}\right)$ and $M^{*}\left(K_{3, n}\right)$ to the problem of blocking $M\left(K_{3, n}\right)$ and $M^{*}\left(K_{3, n}\right)$. The first lemma in this chapter reduces the problem to the problem of bridging the displayed 3-separations of $M$ to the problem of bridging the 3-separations displayed by a restriction of $M$. The second lemma reduces the problem of bridging the 3 -separations in a restriction of $M$ to the problem of bridging 3-separations in a spanning restriction of $M$.

Lemma 7.0.1. There is a function $\int_{7.0 .1}$ such that the following holds. If $n \geq$ $f_{7.0 .1}(t)$ then either

i) $M^{*}$ has a minor $N$ and $N$ has a coindependent set $X$ such that $N \backslash X \cong$ $M^{*}\left(K_{3, t}\right)$ and all 3-separations of $N \backslash X$ displayed by the canonical flower of $M / C \backslash D$ are blocked in $N$, or

ii) $M$ has a restriction $N$ such that $N$ has a paddle with at least t petals.

Proof. By duality we can say that $N_{1}=M^{*} / D \backslash C \cong M^{*}\left(K_{3, n}\right)$. Let the canonical flower of $N_{1}$ be $F$. The set $C$ is a superset of blocking elements for $N_{1}$. If $N$ 
is such that $F$ contains a large set $\mathscr{P}$ of petals such that any 3 -separation of $N_{1}$ displayed by subsets of these petals is blocked in $M^{*} / D$, then we are in case $i$ ) above. Otherwise $M^{*} / D$ has a large induced copaddle with at least $t$ petals. If $M^{*} / D$ has a copaddle with at least $t$ petals then $M \backslash D$ has a large paddle with at least $t$ petals.

Lemma 7.0.2. Let $M$ be a binary matroid with a restriction $N$ such that $N$ has a paddle, $F$, with at least $n$ petals, and every 3-separation displayed by $F$ in $N$ is bridged in $M$. Then $M$ has a minor $M^{\prime}$ such that the following hold.

1. $N$ is a restriction of $M^{\prime}$,

2. $r(N)=r\left(M^{\prime}\right)$,

3. Every 3-separation displayed by $F$ is blocked in $M^{\prime}$.

Proof. Since $N$ is a binary matroid there is a line in $\langle E(M)\rangle$ that spans the common guts of all the 3-separations of $N$ displayed by $F$. Let $\left\{g_{1}, g_{2}\right\}$ be a basis of this line. Let $\widetilde{N}=N+\left\{g_{1}, g_{2}\right\}$ and let $\widetilde{M}=M+\left\{g_{1}, g_{2}\right\}$. Observe that if $(A, B)$ is a 3-separation of $N$ displayed by $F$ then $\left(A \cup\left\{g_{1}, g_{2}\right\}, B\right)$ is a 3-separation of $\widetilde{N}$ and $(A, B)$ is a separation of $\widetilde{N} /\left\{g_{1}, g_{2}\right\}$. Observe that. up to loops, $\widetilde{M} /\left\{g_{1}, g_{2}\right\}$ is connected. Consider $x \in M$ such that $x \notin \operatorname{cl}(E(N) N)$. We can either delete or contract $x$ without unblocking any displayed 3-separations of $N$. For suppose now that both $M \backslash x$ and $M / x$ contain a displayed 3-separation; then $M \backslash x / \operatorname{cl}\left\{g_{1}, g_{2}\right\}$ would not be connected, and $M / x / \operatorname{cl}\left\{g_{1}, g_{2}\right\}$ would not be connected. In other words $M / \operatorname{cl}\left\{g_{1}, g_{2}\right\} / x$ and $M / \operatorname{cl}\left\{g_{1}, g_{2}\right\} \backslash x$ would not be connected. However from this it would follow that $M / \operatorname{cl}\left\{g_{1}, g_{2}\right\}$ is not connected; a contradiction. Therefore $M$ has a minor $M^{\prime}$ that has a restriction $N$ such that $N$ has a maximal paddle with $n$ petals such that every displayed 3-separation of $N$ is blocked in $\mathrm{cl}_{M^{\prime}}(E(N))$, every element of $M^{\prime}$ is in $\mathrm{cl}_{M^{\prime}}\left(E\left(N^{\prime}\right)\right)$ and $N$ is blocked in $M^{\prime}$.

Thus if we have a 4-connected matroid $M$ with an $M\left(K_{3, n}\right)$ or $M^{*}\left(K_{3, n}\right)$-minor, this reduces to the case of blocking a paddle or $M^{*}\left(K_{3, n}\right)$. This gives

Theorem 7.0.3. There is a function $f$ such that if $M$ is a 4-connected binary matroid with an $M\left(K_{3, f(n)}\right)$ or $M^{*}\left(K_{3, f(n)}\right)$ minor, then $M$ must have a minor isomorphic to one of

i) $N\left(K_{3, n}\right)$, 
ii) $M\left(K_{4, n}\right)$,

iii) an n-rung circular ladder,

iv) an n-rung Möbius ladder,

v) the dual of one of the matroids in i)-iv),

vi) $M^{*}\left(K_{3, n}\right)$ blocked by a set $X$ where the crossing graph of $X$ is a path.

Proof. Let $n \geq \int_{7.0 .1}\left(\max \left\{\int_{6.0 .1}(n), \int_{5.0 .1}(n)\right\}\right)$. By Lemma 7.0.1 either

1. $M^{*}$ has a minor $N$ and $N$ has a coindependent set $X$ such that $N \backslash X \cong$ $M^{*}\left(K_{3, m}\right)$ and all displayed 3-separations of $N \backslash X$ are blocked in $N$, or

2. $M$ has a restriction $N$ such that $N$ has a paddle with at least $m$ petals,

where $m \geq \max \left\{\int_{6.0 .1}(n), f_{5.0 .1}(n)\right\}$.

If $M^{*}$ has a minor $N$ and $N$ has a coindependent set $X$ such that $N \backslash X \cong M^{*}\left(K_{3, m}\right)$ and all displayed 3-separations of $N \backslash X$ are blocked in $N$, then the result follows by Theorem 6.0.1.

If $M$ has a restriction $N$ such that $N$ has a paddle with at least $m$ petals, then, by Lemma 7.0.2, $M$ has a minor $M^{\prime}$ such that the following hold.

1. $N$ is a restriction of $M^{\prime}$,

2. $r(N)=r\left(M^{\prime}\right)$,

3. Every 3 -separation displayed by $F$ is blocked in $M^{\prime}$.

The result then follows from Theorem 5.0.1. 


\section{Chapter 8}

\section{Useful Lemmas For Blocking Swirl-Like Pseudo-Flowers}

In Chapter 9 we find unavoidable minors of matroids with blocked swirl-like pseudo-flowers. Before we do this we need to set up some tools. This is the purpose of this chapter.

\subsection{Crossing Graphs for Swirl-Like Pseudo-Flowers}

Recall that a displayed 3-separation in a swirl-like pseudo-flower $F$ is a partition of the petals of $F$ in sets $A$ and $B$ such that $\lambda(A)=2$. Throughout this section we work under the following hypotheses.

- $M_{1}$ is a matroid with a coindependent set $X$ such that $M=M_{1} \backslash X$ has a maximal swirl-like pseudo-flower $F=\left(P_{1}, \ldots, P_{m}\right)$ of order $m$, and

- every 3-separation of $M$ displayed by $F$ is blocked by an element of $X$.

- $\widetilde{M}$ denotes the matroid $M$ extended by the joints of $F$.

Without loss of generality we may assume that $F$ has no clump $C$ with a blocking element, $e$, contained in the closure of $C$, as otherwise we could consider $C \cup e$ to be a petal of a flower $F^{\prime}$ of $M_{1} \backslash(X-e)$ and the pair $M_{1}$ and $F^{\prime}$ would fit the hypotheses of this section. 
In this section we define a graph $(V, E)$ where $V=X$ and there is an edge between a pair of vertices if, and only if, those vertices "cross". We then show that this graph is connected if, and only if, every displayed 3-separation in $F$ is blocked. Once we know the graph is connected we can use known results for unavoidable induced subgraphs to find structure in the arrangement of the blocking elements. Thus our first step will be to give an appropriate definition of when two blocking elements cross and show that the crossing graph behaves as we want it to.

Definition 8.1.1. Two blocking elements $e$ and $f$ of $X$ do not cross in $F$ if there is a displayed 3-separation $(P, Q)$ of $M$ by $F$ such that $e \in \operatorname{cl}(P)$ and $f \in \operatorname{cl}(Q)$.

Definition 8.1.2. Let $B$ be a basis for $\widetilde{M} \backslash X$ that conatains the joints of $F$. A petal $P$ of $F$ contains a representative of a blocking element $x$ in $P$ if the fundamental circuit of $x$ with respect to $B$, denoted $F(x)$, contains an element of $P$. An element $x^{\prime}$ in $\langle P\rangle$ is called the shadow of $x$ on $P$, if the fundamental circuit of $x^{\prime}$ with respect to $B$ in $M$ extended by $x$, denoted $F\left(x^{\prime}\right)$, is equal to $F(x) \cap P$.

Recall that the basepoints of a petal $P_{i}$ are elements of the ambient extended projective space that are the joints of $P_{i}$ or in a triangle with the joints of $P_{i}$. We earlier associated basepoints with petals and sets of petals with blocking elements, now we assign sets of basepoints to blocking elements. Later we will do a similar things with joints.

Definition 8.1.3. Let $x$ be an element that blocks a 3-separation of $M$ displayed by $F$. The basepoints of $x$, denoted $b(x)$ are the basepoints of the petals that contain a representative of $x$.

We also want to associate petals with basepoints.

Definition 8.1.4. The set of petals of a basepoint $b$, denoted $p(b)$, is the set of petals containing $b$ as a basepoint.

Now we shall colour basepoints according to blocking elements as follows:

Definition 8.1.5. Let $B$ be the set of basepoints of $F$. Let $C$ be a set of colours with the property that there is a bijection $\gamma: X \rightarrow C$. A colouring of $F$ is a function $\psi: B \rightarrow \mathscr{P}(C)$ such that $\psi(b)=\{c: c=$ $\gamma(x)$ for some $x$ with a representative in $P(b)\}$ We refer to an element of $\psi(b)$ as a basic colour of $b$. 
The function $\gamma$ is not mathematically interesting. However, it helps with the colouring analogy we use later and is thus helpful when we draw pictures in later sections.

Definition 8.1.6. We say that two colours, $r$ and $g$, assigned to elements of a cyclically ordered set $S$ alternate if there are distinct elements $a_{i}, a_{j}, a_{k}$ and $a_{l}$ of $S$ such that $[i, j, k, l]_{i}$ and $r$ is a colour of $a_{i}$ and $a_{k}$ and $g$ is a colour of $a_{j}$ and $a_{l}$ or vice versa. If $r$ and $g$ alternate in a colouring, $\psi$, of $S$ then we say that $\psi$ is an alternating colouring for $r$ and $g$.

Definition 8.1.7. Let $c: S \rightarrow \mathscr{P}(C)$ be a colouring of a cyclically ordered set $S$. We say that two elements $r$ and $g$ colour-cross if there is a alternating colouring for $r$ and $g$ in $c$.

We want to say two blocking elements cross if, and only if, they either contain representatives in the same petal or their associated colours colour-cross. However with the definition of colouring for a swirl-like pseudo-flower we currently have this is not true. For example if we have the following picture

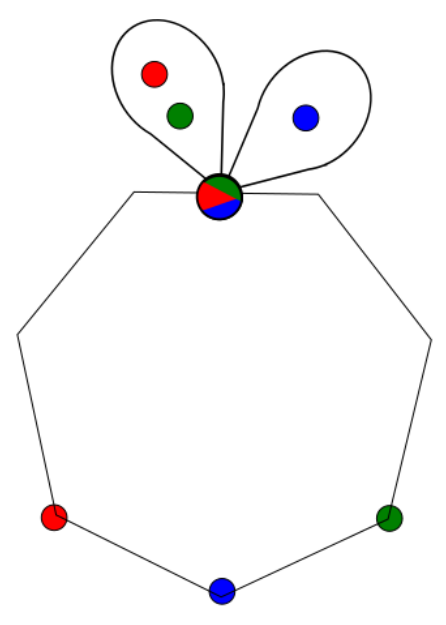

then, none of red, green and blue colour-cross. However, when viewed as blocking elements we can see that one of red and blue crosses green. This leads us to define auxiliary colours.

\section{Definition 8.1.8.}


i) Let $\alpha: C \times C \rightarrow E \cup \emptyset$ where $E$ is a set of colours with the property that $C \cap E=\emptyset$ with $\alpha\left(c_{i}, c_{j}\right)=\left\{\begin{array}{l}\emptyset \text { if there is no 2-petal containing representatives of both } \gamma^{-1}\left(c_{i}\right) \text { and } \gamma^{-1}\left(c_{j}\right) \\ e_{i j} \text { otherwise, where } e_{i j}=e_{k l} \text { if, and only if, }\{i, j\}=\{k, l\} .\end{array}\right.$

This function assigns a unique auxiliary colour to pairs of colours if those colours appear in the same 2-petal.

ii) An auxiliary colouring of a swirl-like pseudo-flower is a function $\chi: B \rightarrow$ $\mathscr{P}(C \cup E)$ such that $\chi(b)=\psi(b) \cup\{c: c \in E$ and $c$ is an auxiliary colour of a pair $\left(c_{i}, c_{j}\right)$ with at least one of $\left\{c_{i}, c_{j}\right\}$ in a petal of $\left.b\right\}$.

iii) The elements of $\chi(b)$ are the colours of $b$.

iv) Let $\omega: X \rightarrow C \cup E$ be a function such that $\omega(x)=\gamma(x) \cup\left\{c \in E:\left(\gamma(x), c_{i}\right)=\right.$ $c$ for some $\left.c_{i} \in C\right\}$. We call the elements of $\omega(x)$ the colours of $x$.

Recall that $F$ has no clump $C$ with an element of $x$ contained in $\bigcup C$.

Lemma 8.1.9. If two blocking elements $x$ and $y$ cross, then either $x$ and $y$ are such that there is a petal containing a representative of both $x$ and $y$, or some colour of $x$ colour-crosses some colour of $y$.

Proof. If representatives of $x$ and $y$ appear in the same petal then $x$ and $y$ cross.

Suppose there is no petal containing representatives of both $x$ and $y$ and suppose there is no colour of $x$ that colour-crosses a colour of $y$. Then for any pair of colours, $(r, g)$ where $r \in \omega(x)$ and $g \in \omega(y)$, we cannot find an alternating colouring with respect to $r$ and $g$. This means there are two sets, $B_{1}=\left\{b_{i}, \ldots, b_{m}\right\}$ and $B_{2}=\left\{b_{j}, \ldots, b_{n}\right\}$, of consecutive basepoints, one of which contains all basepoints assigned colour $r$ and the other containing all basepoints assigned colour $g$. Since $r$ and $g$ do not cross, $\left|B_{1} \cap B_{2}\right| \leq 2$. If $r$ and $g$ do not cross and $x$ and $y$ do not have representatives in the same petal as each other the only way $x$ and $y$ can cross is if the following hold:

i) all representatives of $x$ and $y$ are in distinct 2-petals that share a basepoint, and 
ii) there is a representative of an element $z$ contained in a petal that contains a representative of one of $x$ and $y$, and

iii) if $x$ and $z$ have representatives in the same petal then there are basepoints $b_{i}, b_{j}, b_{k}$ and $b_{l}$ with $\left[b_{i}, b_{j}, b_{k}, b_{l}\right]_{b_{i}}$ such that the following hold.

a) $r, g$ and $\gamma(z)$ colours of $b_{i}$,

b) $r$ a colour for exactly one of $b_{j}$ and $b_{l}$ and $\gamma(z)$ a colour for the other, and

c) $g$ a colour of $b_{k}$,

and

iv) if $y$ and $z$ have representatives in the same petal there are basepoints $b_{i}, b_{j}, b_{k}$ and $b_{l}$ with $\left[b_{i}, b_{j}, b_{k}, b_{l}\right]_{b_{i}}$ such that the following hold.

a) $r, g$ and $\gamma(z)$ colours of $b_{i}$,

b) $g$ a colour for exactly one of $b_{j}$ and $b_{l}$ and $\gamma(z)$ a colour for the other, and

c) $r$ a colour of $b_{k}$.

However in this case there is either an auxiliary colour for $(x, z)$ and this colour colour-crosses $g$, or an auxiliary colour of $(y, z)$ that colour-crosses $r$. This is a contradiction since this means that some colour of $x$ colour-crosses a colour of $y$.

The following lemma is essentially the converse of Lemma 8.1.9.

Lemma 8.1.10. If $r, g \in C \cup E$ colour-cross then $x$ and $y$ cross for some $x, y \in X$ with $\omega(x)=r$ and $\omega(y)=g$.

Proof. If $r, g \in C$ then this is clear. Suppose that exactly one of $r$ and $g$ is in $E$. Without loss of generality let this be $r$. Then there are at least two elements $x_{1}$ and $x_{2}$ that both have representatives in some 2-petal, $P_{i}$, of $F$, and these elements are assigned the unique auxiliary colour $r$. Therefore we either see an alternating colouring from $P_{i}$ with respect to $r$ and $g$ starting with $r$ or an alternating colouring from $P_{i}$ with respect to $r$ and $g$ starting with $g$. In the first case it is easy to see that there would be an alternating colouring from $P_{i}$ with respect to $c$ and $g$ for some $c \in C$. Therefore assume that we are in the second case. Since $r$ is an auxiliary colour, $r=\alpha\left(x_{1}, x_{2}\right)$ for some unique $x_{1}, x_{2}$. Suppose that $x_{1}$ does not cross $y$. 
Then we can find a displayed 3-separation with $x_{1}$ on one side and $y$ on the other. But then $x_{2}$ crosses this separation. The argument is similar when both $r$ and $g$ are elements of $E$ and is left to the reader.

Definition 8.1.11. We say that two colours $c$ and $d$ transitively colour-cross if we can find a path of colours $c, c_{1}, \ldots, c_{k}, d$ so that $c$ crosses $c_{1}$ and $c_{k}$ crosses $d$ and for every $i \in\{2, \ldots, k-1\} c_{i}$ crosses $c_{i-1}$ and $c_{i+1}$. We say that two elements $x, y \in X$ transitively cross if a colour associated with $x$ and a colour associated with $y$ transitively colour-cross.

Definition 8.1.12. The crossing graph of the blocking elements $X$ of $F$ is the graph $G=(V, E)$ where $V=X$ and there is an edge between two elements $x$ and $y$ of $V$ if, and only if, $x$ and $y$ cross.

This means that if two vertices $x$ and $y$ are joined by an edge then either:

i) there is some petal in $F$ containing both and representative of $x$ and a representative of $y$ or,

ii) some colour of $x$ crosses some colour of $y$.

It is clear that if there is a path between two vertices $x$ and $y$ of the crossing graph then $x$ and $y$ transitively cross.

- Throughout the remainder of this chapter we use $J$ to denote the joints of swirl-like pseudo-flower $F$.

Theorem 8.1.13. Let $H$ be the crossing graph of the set $X$ of blocking elements of $F$. The graph $H$ is connected if, and only if, every displayed 3-separation of $F$ is blocked.

Proof. Suppose that $H$ is not connected. Consider a graph $\widetilde{H}$ that is obtained by adding edges to $H$ to obtain a graph that has exactly two connected components. If $F$ is not blocked when we add the crossings induced by the edges of $E(\widetilde{H})$ then $F$ was not blocked originally. Therefore for the remainder of this proof we may assume without loss of generality that $H$ has exactly two connected components $H_{1}$ and $H_{2}$. For this proof we want to add a new level of colouring which we shall call the component colouring. The component colouring of the basepoints of $F$ is an assignment of one or both of $r, g$ to the basepoints of $F$ as follows: 
i) if $x \in H_{1}$ is such that $\gamma(x)=c$ then for every $j \in J$ with $c \in \psi(j)$ assign colour $r$ to $j$, and

ii) if $x \in H_{2}$ is such that $\gamma(x)=c$ then for every $j \in J$ with $c \in \psi(j)$ assign colour $g$ to $j$,

where $r, g$ are distinct from any element of $C \cup E$. The component colour of a basepoint is the value it is assigned in the component colouring of $F$. Let $R$ be the set of joints with component colour $r$ and let $G$ be the set of joints with component colour $g$. Note that $R \cap G$ may be non-empty.

Claim 8.1.14. $R$ and $G$ are consecutive sets.

Proof. Since any pair of colours in $R$ transitively cross and any pair of colours in $G$ transitively cross, if $R$ and $G$ were not consecutive sets then we would have a vertex in $H_{1}$ crossing a vertex in $H_{2}$, a contradiction.

Claim 8.1.15. Let $C=\left\{b_{i}, \ldots, b_{m}\right\}$ be the set of basepoints with component colour $c$ and let $C_{1}=\left\{b_{j}, \ldots, b_{k}\right\}$ be a consecutive set of basepoints with component colour $c$ such that $C-C_{1}=\left\{b_{1}, \ldots, b_{j-1}, b_{k+1}, \ldots, b_{m}\right\}$ is nonempty. There is some colour that appears as a colour of both a basepoint in $C_{1}$ and a basepoint $b_{a}$ in $C-C_{1}$ with $a \notin\{j-1, k+1\}$.

Proof. This holds since any element of $B$ transitively crosses some other element of $B$ for $B \in\{R, G\}$.

Claim 8.1.16. $|R \cap G| \leq 2$ and if $|R \cap G|=2$ then the joints in $R \cap G$ are not adjacent.

Proof. First note that $|R| \geq 2$ as if $|R|<2$ then the elements of $H_{1}$ would not block any displayed three-separation. Similarly $|G| \geq 2$. Suppose we have a consecutive set of basepoints coloured both $r$ and $g$ and this set contains more than one basepoint. Let this consecutive sequence of basepoints be $\left\{b_{i},,, . b_{k}\right\}$ and let elements $b_{1}, \ldots, b_{i-1}$ be in $R-G$ and $b_{k+1}, \ldots, b_{m}$ be in $G-R$. Note that $m$ may not be equal to the number of basepoints and if it is not then we have a second consecutive subset with elements in $R \cap G$. We know that for any basepoint $b_{l} \in\left\{b_{i}, \ldots, b_{k}\right\}$ we can find some colour $o$ such that $o$ is assigned both to $b$ and some element of $V\left(H_{2}\right)$. Choose any such pair with the restriction that $l \in\{i, \ldots, k-1\}$. There is some $b_{p}$ with $p \notin\{1, \ldots, i, l-1, l+1\}$ that is also assigned colour $o$. Without 
loss of generality let $\left[b_{1}, b_{l}, b_{p}\right]$. Now consider the consecutive subset of $R$ that is contained in $\left\{b_{p}, \ldots, b_{l+1}\right\}$. By Claim 8.1.16 above there is some basepoint in this set assigned colour $w$ where $w \in \omega(x)$ for some $x \in V\left(G_{1}\right)$ with the property that some element in $\left\{b_{1}, \ldots, b_{l-1}\right\}$ is also assigned colour $w$. This means that $o$ and $w$ cross which contradicts the fact that $G$ is not connected.

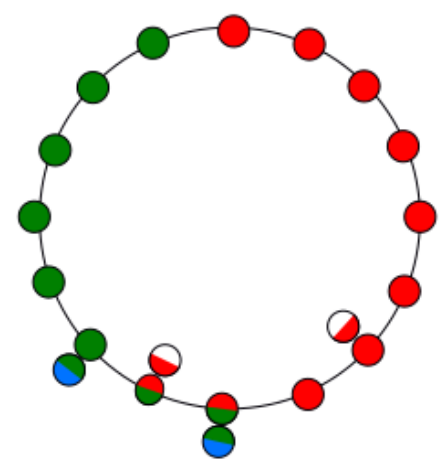

This means that no colour of an basepoint of $R$ crosses a colour of a basepoint in $G$ and so we have a displayed 3-separation in the swirl-like pseudo-flower.

Suppose that we have a 3-separation displayed by $F$. If we restrict our attention to the colours in $C$ we see that there is a partition $\left[C_{1}, C_{2}\right]$ of $C$ so that no colour of $c_{1}$ crosses a colour of $c_{2}$. Let the blocking elements assigned $C_{1}$ form a set $V_{1}$ of vertices of $H$. It is easy to see that there is no edge between a vertex of $V_{1}$ and a vertex of $V(H)-V_{1}$.

Finally recall Lemma 2.4.2. This tells us that there exists a function $\sqrt{2.4 .2}$ such that, if $G$ is a connected graph $G$ with at least $\sqrt{2.4 .2}$ vertices, then there an induced subgraph of $G$ on $n$ vertices that is either a path, a complete graph, or a star.

This gives structure to the blocking elements of $F$, as we shall see shortly.

\subsection{Structuring the Crossing Elements}

Throughout this section we work under the following hypotheses. 
- Let $M$ be a matroid with a coindependent set $X$ such that $M \backslash X$ has a maximal swirl-like pseudo-flower $F=\left(P_{1}, \ldots, P_{m}\right)$ of order $n$, and

- every 3-separation of $M \backslash X$ displayed by $F$ is blocked by an element of $X$.

This means that either we have a single element of $X$ that blocks a lot of 3separations, or $X$ has many elements and, since every displayed 3-separation of $F$ is blocked, by Theorem 8.1 .13 the crossing graph is connected. We formalise this below.

Lemma 8.2.1. There is a function $\sqrt{8.2 .1}$ such that the following holds. Suppose that $M$ has $\sqrt{8.2 .1}(m, k) 3$-separations displayed by $F$. Then either $|X| \geq m$ or there is some $x \in X$ that blocks at least $k$ displayed 3-separations.

Proof. Observe that this follows when $f \frac{8.2 .1}{(m, k)}=k(m-1)$.

The following lemma is clear and the proof is omitted.

Lemma 8.2.2. Suppose that $F$ has $\int 8.2 .1(2.4 .2(m), k)$ displayed 3-separations. Then there is either some $x \in X$ that blocks at least $k$ displayed 3-separations of $M$ by $F$, or there is an induced subgraph of the crossing graph of the blocking elements of $F$ in $M$ that is either a path, a star or a complete graph with at least $m$ vertices.

Definition 8.2.3. We say that $F$ is partially blocked in $M$ if there is some nonempty $X^{\prime} \subseteq X$ in which some of the 3-separations of $M$ displayed by $F$ are blocked.

The following lemma is trivial.

Lemma 8.2.4. Let the crossing graph of $X$ in $F$ be $G$ and suppose $G^{\prime}$ is an induced subgraph of $G$. If $X^{\prime}=V\left(G^{\prime}\right)$, then $N=M \backslash\left(X-X^{\prime}\right)$ such that the following hold.

i) $N$ has a minor $N \backslash X^{\prime}$ with a swirl-like pseudo-flower $F$,

ii) $F$ is partially blocked in $N$ by $X^{\prime}$, and

iii) elements $x$ and $y$ of $X^{\prime}$ cross in $F$ in $N$ if, and only if, they cross in $F$ in $M$.

For the remainder of this section we work under the following additional hypotheses. 
- $|X| \geq s$,

- The crossing graph of $X$ with respect to $F$ in $M$ is $G$,

- $G^{\prime}$ is an induced subgraph of $G$ with vertex set $X^{\prime}$ that is either a path, a star or a complete graph and $\left|X^{\prime}\right|=m$.

Recall that if $M$ has a swirl-like pseudo-flower $F$, there is a minor, $M^{\prime}$, of $M$ obtained by removing petals of $F$ that also has a swirl-like pseudo-flower.

Lemma 8.2.5. Suppose that the displayed 3-separations of $M$ by $F$ are partially blocked by $X^{\prime}$. There is a minor, $M^{\prime}$, of $M$ such that $X^{\prime}=E\left(M^{\prime}\right) \cap X$, that $M^{\prime} \backslash X^{\prime}$ has swirl-like pseudo-flower $F^{\prime} \subseteq F$ and that every displayed 3-separation of $F^{\prime}$ is blocked by an element of $X^{\prime}$. Moreover, all elements of $X^{\prime}$ block some displayed 3-separation of $M^{\prime}$ by $F^{\prime}$.

Proof. Partition the joints of $F$ into two sets $\left[J_{1}, J_{2}\right]$, where $J_{1}$ is the set of joints that appear as in $J(x)$ for some $x \in X^{\prime}$. We show that there is minor $M^{\prime}$ of $M$ with pseudo-flower minor, $F^{\prime} \subseteq F$, in which any $j$ in $J_{1}$ is a joint of a petal in $F^{\prime}$. Suppose there are two adjacent joints, $j_{i}$ and $j_{i+1}$ in $J_{2}$. Then there is some rim element $r_{i}$ between $j_{i}$ and $j_{i+1}$ and there is no $x \in X^{\prime}$ with an element of $F(x)$ in a petal with $r_{i}$ as a basepoint. Therefore we can contract $r_{i}$. Suppose there is a joint $j_{2}$ such that $j_{2} \in J_{1}$ and $j_{1}, j_{3} \in J_{2}$. Then $r_{1}$ and $r_{2}$ are not basepoints of any petals containing an element of $F(x)$. Therefore we can remove every petal with $r_{1}$ or $r_{2}$ as a basepoint. The flower is then such that every 3-separation is blocked by an element of $X^{\prime}$ and all elements of $X^{\prime}$ block some displayed 3-separation.

The following theorem follows from Theorem 8.1.13, Lemma 2.4.2, Lemma 8.2.2 and Lemma 8.2 .5 .

Theorem 8.2.6. There is a function 8.2 .6 such that the following holds. Let $M$ be a matroid with a coindependent set $X$ such that $M \backslash X$ has a maximal swirllike pseudo-flower $F=\left(P_{1}, \ldots, P_{m}\right)$ of order $n$, and suppose every 3-separation of $M \backslash X$ displayed by $F$ is blocked by an element of $X$. If $n \geq \int_{8.2 .1}\left(f_{2.4 .1}(t), k\right)$ there is a minor $M^{\prime}$ of $M$ with coindependent set $X^{\prime}=X \cap E\left(M^{\prime}\right)$ such that the following holds.

i) $M^{\prime} \backslash X^{\prime}$ has a swirl-like pseudo-flower $F^{\prime}$, 
ii) every 3-separation of $M^{\prime}$ displayed by $F^{\prime}$ is blocked by some element $x \in X^{\prime}$,

iii) either $\left|X^{\prime}\right|=1$ and $M^{\prime} \backslash X^{\prime}$ has $k$ 3-separations displayed by $F^{\prime}$, or the crossing graph of $X^{\prime}$ with respect to $F^{\prime}$ in $M^{\prime}$ is either a star, a path or a complete graph on at least $t$ elements.

Proof. Let $n \geq \int$ 8.2.1 $\left(\int 2.4 .2(t), k\right)$ By Lemma 8.2.2 either there is some $x \in X$ that blocks $k$ 3-separations of $M \backslash X$ displayed by $F$, in which case it is easy to see that the theorem holds, or the crossing graph of $X$ in $M$ has an induced subgraph that is either a star, path or complete graph on at least $t$ vertices. Let $X^{\prime} \subseteq X$ be the vertices in such an induced subgraph. Then by Lemma 8.2 .5 there is a minor $M^{\prime}$ of $M$ such that $M^{\prime} \backslash X^{\prime}$ has a swirl-like pseudo-flower $F^{\prime}$ in which every displayed 3 -separation is blocked by some element of $X^{\prime}$ and the crossing graph of $X^{\prime}$ in $M^{\prime}$ is either a star, path, or complete graph on at least $t$ elements.

This means that we can find some minor $M^{\prime}$ of $M$ with a swirl-like pseudo-flower $F^{\prime} \subseteq F$ such that $F^{\prime}$ is blocked by a single element, or the blocking elements of $F^{\prime}$ are:

i) a large set of blocking elements every member of which crosses every other member, or

ii) a large set of blocking elements none of which cross, along with a single blocking element which crosses every member of this set, or

iii) a set of elements in which all but two cross exactly two members of the set and the remaining two elements each cross exactly one member of the set and do not cross each other,

We call these the complete graph case, the star case and the path case respectively.

\subsection{Useful Lemmas For Reducing Petals Containing Representatives of Two Blocking Elements}

When a swirl-like pseudo-flower is blocked in a path-like way sometimes a petal $P$ contains representatives of two blocking elements $x_{1}$ and $x_{2}$. We want to reduce the size of the petal as much as possible without losing joints, or the fact that $x_{1}$ 
and $x_{2}$ contain representatives in $P$. In this section we look at finding minors of connected matroids using three or four particular elements. This will be useful later on when we are blocking swirl-like pseudo-flowers in a path-like way.

The next two lemmas are trivial.

Lemma 8.3.1. If $M$ is a connected matroid containing elements $a, b, c$ then there is a minor of $M$ in which either $\{a, b, c\}$ form a triangle or $\{a, b, c\}$ are parallel.

Lemma 8.3.2. Let $a$ and $b$ be elements of a connected binary matroid $M$. If $a \in \operatorname{cl}(E(M)-\{a, b\})$ and $b \in \operatorname{cl}(E(M)-\{a, b\})$ there is a minor of $M$ that is a parallel class containing $a, b$ and some $e \in(E(M)-\{a, b\})$.

Lemma 8.3.3. Let $M$ be a connected binary matroid for rank at least 2 with $r, g, x \in E(M)$. Suppose there is no 2-separation $(A, B)$ in $M$ with $r \in A$ and $g \in B$. Then there is a minor of $M$ of one of the following forms:
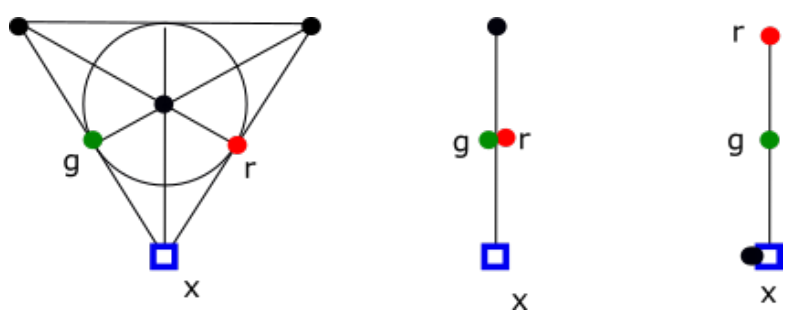

Proof. We first show that there is a 3-connected minor, $N$, of $M$, and then use Lemma 2.1 .16 to analyse the various possibilities for $N$. First suppose that there is a 2-separation $(A, B)$ in $M$ with $r, g \in A$ and $x \in B$. By Tutte's Linking Theorem there is a minor, $M^{\prime}$, of $M$ such that $E\left(M^{\prime}\right)=A \cup\{x\}$, and $M\left|A=M^{\prime}\right| A$, and $x \in \mathrm{cl}_{M^{\prime}}(A)$. This means that, for the remainder of the proof, we may assume that no such 2-separation exists. Now suppose that there is a 2-separation $(A, B)$ in $M$ with $r \in A$ and $x, g \in B$. By Tutte's Linking Theorem there is a minor $M^{\prime}$ of $M$ such that $E\left(M^{\prime}\right)=A \cup\{x\}, M\left|B=M^{\prime}\right| B$ and $r \in \mathrm{cl}_{M^{\prime}}(B)$. It is trivial to see that if $(A, B)$ is a 2-separation of $M$ with $r, g, x \in A$, there is a minor $M^{\prime}$ of $M$ on $A \cup b$ for some $b \in B$, where $M^{\prime}|A=M| A$ and $b \in \mathrm{cl}_{M^{\prime}}(A)$. Thus we obtain a 3-connected minor of $M$ of one of the following forms, where the red points indicate $r$ or $g$ and the green points the other and the blue square represents $x$. 

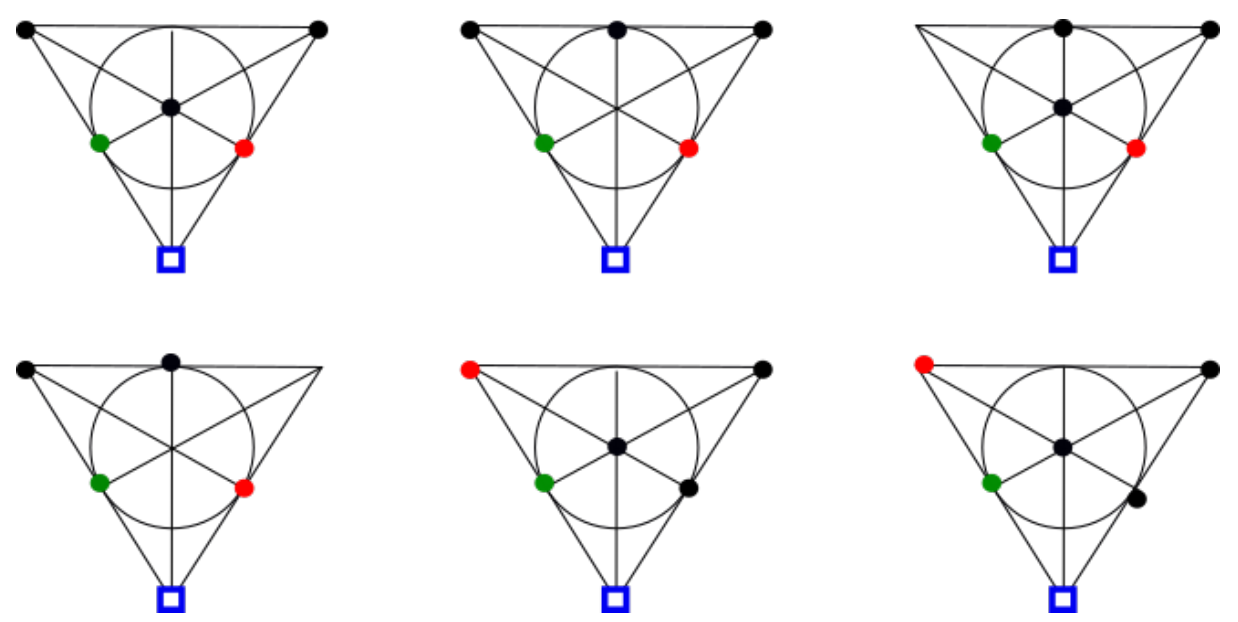

A case analysis of the above possibilities then gives the required minors.

The next two lemmas are immediate consequences of Lemma 8.3.3. They are very similar but one will be useful when the petal we are reducing the size of is joint-based and the other will be useful when the petal we are reducing the size of is rim-based.

Lemma 8.3.4. Let $M$ be a connected matroid with $r, g, x \in E(M)$. Suppose there is no 2-separation in $M$ with $r$ on one side and $g$ on the other. Then there is a minor of $M$ of one of the following forms:
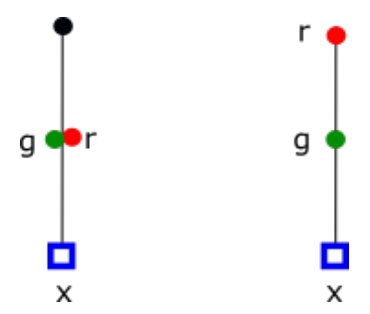

Lemma 8.3.5. Let $M$ be a connected binary matroid with $r, g, x \in E(M)$. Suppose there is no 2-separation in $M$ with $r$ on one side and $g$ on the other. There is a minor of $M$ of one of the following forms:

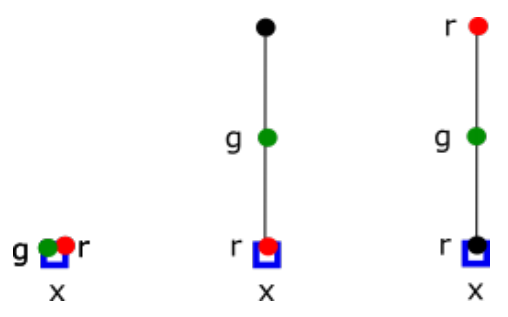


Lemma 8.3.6. Let $M$ be a binary matroid that is minimal with respect to the following properties:

i) $M$ is connected,

ii) $M$ contains elements $x, y, r, g$,

iii) $\{x, y\}$ is independent as are $\{x, g\}$ and $\{r, y\}$,

iv) $M$ has no 2-separation $A, B$ with $x, g \in A$ and $y, r \in B$.

Then $M$ is isomorphic to one of the following: 

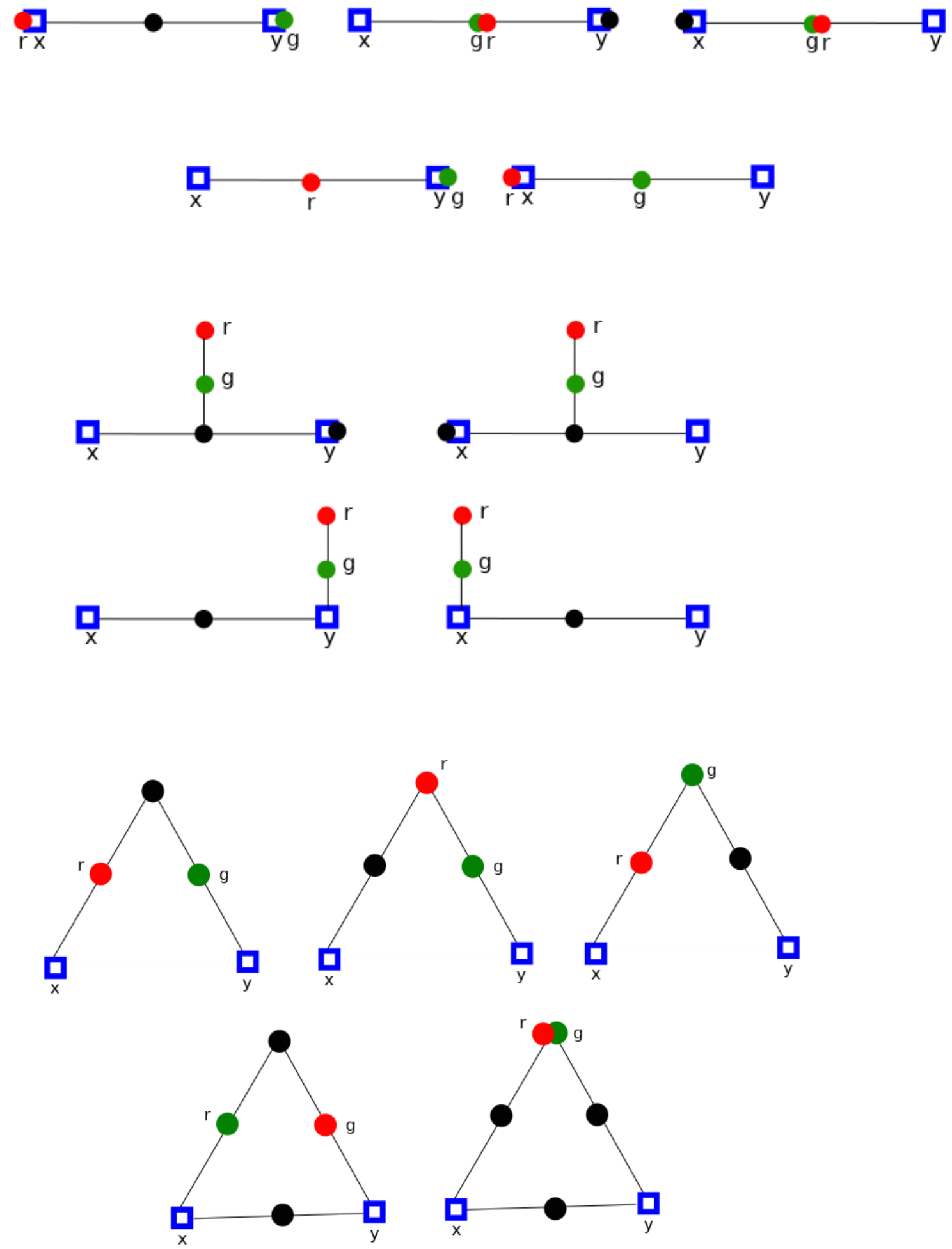

Proof. The proof of this theorem splits into various claims. 
Claim 8.3.7. $M$ is 3-connected up to series pairs and parallel pairs. Moreover, if $M$ contains a series or parallel pair $\{a, b\}$ then $\{a, b\} \in$ $\{\{r, g\},\{x, r\},\{y, g\}\}$.

Proof. Suppose that $M$ has a 2-separation $(A, B)$.

Suppose all of $x, y, r, g \in A$. Then there is some minor $M^{\prime}$ of $M$ obtained by deleting $B^{\prime}$ and contracting $B^{\prime \prime}$, for $B^{\prime}, B^{\prime \prime} \subseteq B$ of the following form:

1. $\left(B-\left(B^{\prime} \cup B^{\prime \prime}\right)\right)=e$,

2. $M^{\prime}|A=M| A$, and

3. $e \in \mathrm{cl}_{M^{\prime}}(A)$.

The matroid $M \backslash B^{\prime} / B^{\prime \prime}$ is a matroid satisfying i)-iii) contradicting the minimality of $M$. Clearly we can apply the same argument if $x, y, r, g \in B$.

Suppose exactly one of $x, y, r, g$ is contained in $A$. Suppose the element in $A$ is $r$. By Tutte's linking theorem, there is a minor $M^{\prime}$, with the property that $E\left(M^{\prime}\right)=$ $E(B) \cup r, M^{\prime}|B=M| B$ and $r \in \mathrm{cl}_{M^{\prime}} B$. This minor satisfies conditions i)-iii) unless $r$ is parallel to $y$ in $M^{\prime}$. The only way $y$ can be parallel to $r$ in $M^{\prime}$ is if $y \in \operatorname{cl}(A) \cap \operatorname{cl}(B)$ which is a contradiction as this would mean $M$ had a 2 separation with $r, y$ on one side and $g, x$ on the other. Therefore $r$ will not be parallel to $g$ in $M^{\prime}$.

We can apply a similar argument if exactly one of $g, x$ or $y$ is in $A$.

Suppose exactly two of $r, g, x, y \in A$ and call these two elements $e, f$. By Lemma 8.3 .1 there is a minor $M^{\prime}$ of $M$ such that $M^{\prime}|B=M| B$, and $E(M)-$ $E\left(M^{\prime}\right)=\{e, f\}$, where $\{e, f\}$ is either a series pair with basepoint in $\operatorname{cl}_{M^{\prime}}(B)$, or $\{e, f\}$ is a parallel pair in $\mathrm{cl}_{M^{\prime}}(B)$. This minor satisfies i)-iii) unless $x, y \in A$, $x, g \in A$ or $y, r \in A$. If $x, g \in A$ then there was a 2-separation in $M$ with $x, g$ in one side and $r, y$ in the other, a contradiction. Similarly we may assume $y, r \notin A$.

Suppose $x, y \in A$. Then $r, g \in B$ and so there is a minor $M^{\prime}$ such that

1. $\left(B-\left(B^{\prime} \cup B^{\prime \prime}\right)\right)=\{r, g\}$,

2. $M^{\prime}|A=M| A$,

3. $\{r, g\}$ is a series or parallel pair,

4. if $\{r, g\}$ is in series then the basepoint of $\{r, g\}$ is in $\langle A\rangle$, and 
5. if $\{r, g\}$ is in parallel then $\{r, g\} \in \mathrm{cl}_{M^{\prime}}(A)$.

Claim 8.3.8. If $M$ contains two series pairs, two disjoint parallel pairs or one series and one parallel pair then $M$ is one of the matroids described in the statement of the lemma.

Proof. By Claim 8.3.7 we may assume that $M$ is connected up to series and parallel pairs and if $a, b$ is in series or parallel in $M$ then $\{a, b\} \in\{\{r, g\},\{x, r\},\{y, g\}\}$. Suppose $M$ is not isomorphic to one of the matroids given in the statement of the lemma.

Suppose $M$ has two parallel pairs. Then these parallel pairs are $\{x, r\}$ and $\{y, g\}$. Since $M$ is connected there is a minor $M^{\prime}$ of $M$ so that $\operatorname{si}\left(M^{\prime}\right)=U_{2,3}$ and $\{x, r\}$ are parallel and $\{y, g\}$ are parallel. Therefor $M$ must be such that $\operatorname{si}\left(M^{\prime}\right)=U_{2,3}$ and $\{x, r\}$ are parallel and $\{y, g\}$ are parallel.

Suppose $M$ has a series pair and a parallel pair. Without loss of generality we may assume that the series pair is $\{x, r\}$ and the parallel pair is $\{y, g\}$. By Tutte's Linking Theorem we can find a minor $M^{\prime}$ of $M$ such that $M^{\prime} \backslash y \cong U_{2,3}$ and $y$ and $g$ are parallel.

Suppose $M$ has two series pairs. These must be $\{x, r\}$ and $\{y, g\}$. Let $A=(E(M)-$ $\{x, y, r, g\})$. The basepoints of $\{x, r\}$ and $\{y, g\}$ must be contained in the closure of $A$. For suppose not, then $r(E(M)-\{r, y\})=r(A)+2$ and $r(M) \geq r(A)+3$. This means that if the basepoints of $\{x, r\}$ and $\{y, g\}$ are not contained in $\operatorname{cl}(A)$ then $\lambda(\{x, r\})=1$, a contradiction. Let the basepoint of $\{x, r\}$ be $a$ and the basepoint of $\{y, g\}$ be $b$. Either there is some element of $E(M)$ parallel to $a$ and $b$ or by Lemma 2.1.16 $M \mid(A \cup\{a, b\})$ has an $M\left(K_{4}\right)$-minor containing $a$ and $b$. This means that we have the following picture:
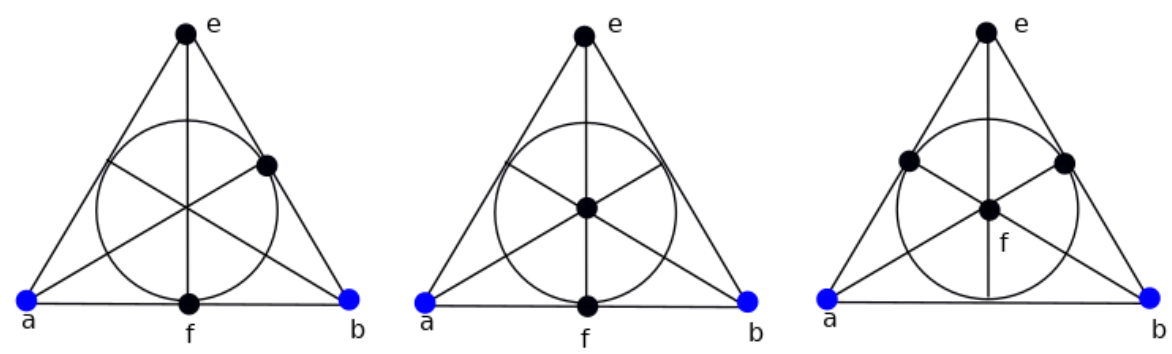
Contracting $e$ and $f$ and replacing $a, b$ with the original series pairs then shows that $M$ has the minor of the form given below:

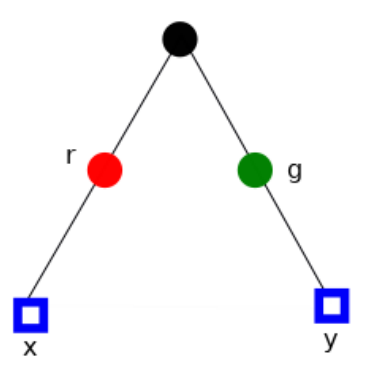

Claim 8.3.9. If $\{r, x\}$ or $\{g, y\}$ is a series pair in $M$ or parallel pair in $M$, then $M$ is isomorphic to one of the matroids described in the statement of the lemma.

Proof. Suppose not and assume that $\{r, x\}$ is a series pair or a parallel pair. If $\{g, y\}$ is a series or parallel pair and the result follows immediately from Claim 8.3.8. Assume $\{y, g\}$ is not a series or parallel pair and let $a$ be the basepoint of $\{r, x\}$ if $r, x$ are in series or an element parallel to $\{r, g\}$ if $r, g$ are in parallel (note $a$ may not be in $E(M)$ ). By Lemma 2.1 .16 there is an $M\left(K_{n}\right)$-minor of $M$ using $a, g, y$. This means that there is a minor of $M$ isomorphic to one of the following:

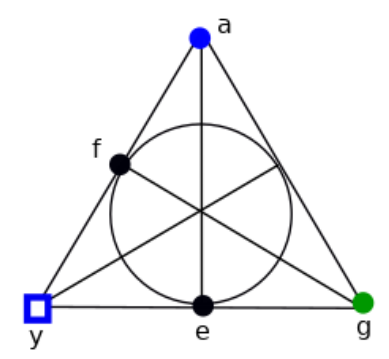

A

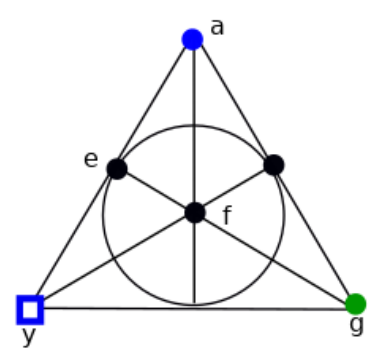

B

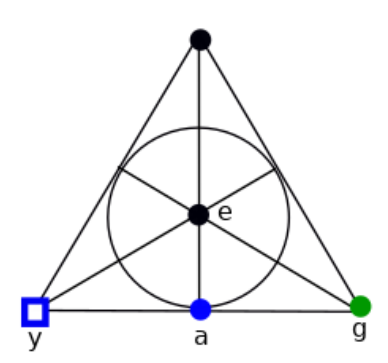

C

If $r, x$ is a series pair then contract $e$ and $f$ in $A$, and $e$ in $B$ and $C$ to get 

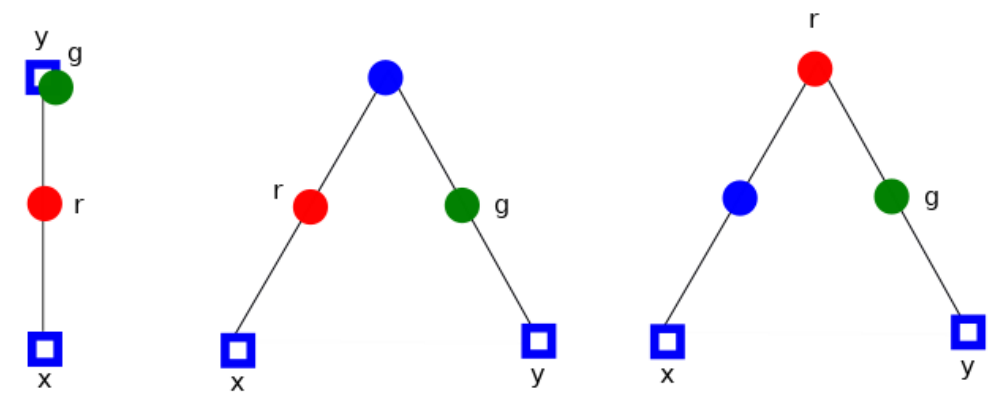

respectively.

Suppose $\{r, x\}$ is a parallel pair then contract $e$ in $A, f$ in $B$ and $e$ in $C$ to get one of:
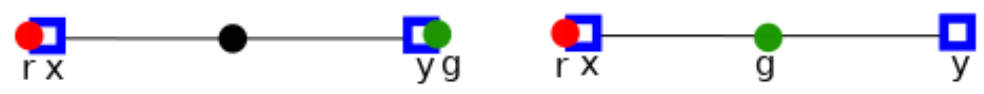

Claim 8.3.10. If $M$ is not 3-connected then $M$ is isomorphic to one of the matroids described in the statement of the lemma.

Proof. By Claim 8.3.8 we may assume that $M$ contains at most one series or parallel pair. By Claim 8.3.9 we may assume that the series or parallel pair is $\{r, g\}$. If $\{r, g\}$ is a series pair then let the basepoint of $\{r, g\}$ be $a$ and if $\{r, g\}$ is a parallel pair add an element $a$ in parallel with $\{r, g\}$. Note that $a$ may not be in $M$. Let $M_{1}$ be the matroid obtained from $M$ be replacing $\{r, g\}$ with $a$. Since $M_{1}$ is 3 -connected we can find an $M\left(K_{3, n}\right)$-minor of $M_{1}$ using $\{a, x, y\}$. This means that up to symmetry $M_{1}$ has a minor of one of the forms below: 


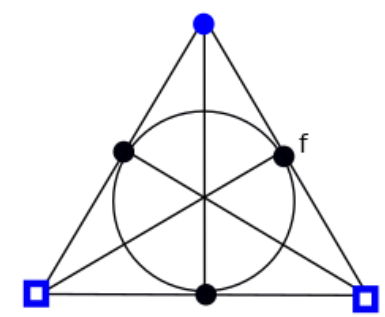

A

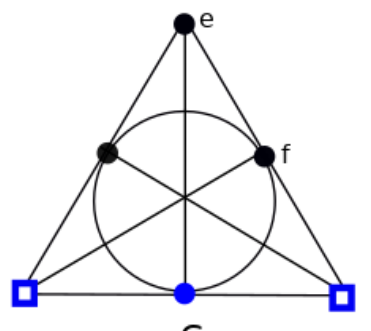

C

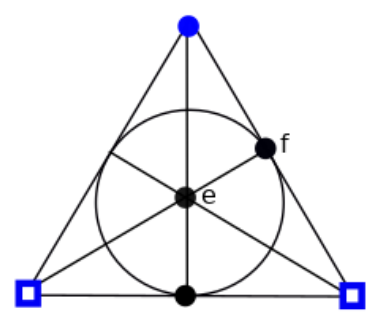

B

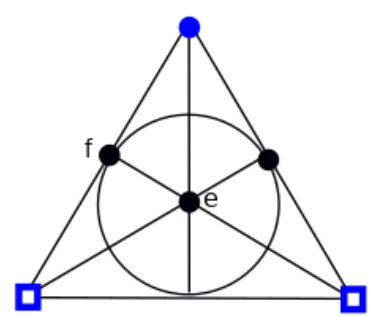

D

where the blue squares represent $x$ and $y$ and the blue circle represents $a$. Consider case A. If $r, g$ are in parallel then, when we replace $a$ by the parallel pair $\{r, g\}$, this is one of the matroids described in the statement of the lemma. If $r, g$ are in series then contracting $f$ and replacing $a$ with the series pair with $\{r, g\}$ gives one of the minors described in the statement of the lemma. In the remaining cases if we contract $e$ when $r$ and $g$ are in parallel and replace $a$ by $\{r, g\}$ we get one of the matroids described in the statement of the lemma. If $\{r, g\}$ are a series pair then contracting $f$ and replacing $a$ with the series pair $\{r, g\}$ gives one of the minors described in the statement of the lemma.

For the remainder of this proof we assume that $M$ is 3-connected.

Claim 8.3.11. $M$ has rank at most 3 .

Proof. Suppose $r(M)>3$. Then there is some element $e$ that is not in $\operatorname{cl}(g, x), \operatorname{cl}(r, y)$ or $\operatorname{cl}(x, y)$. This element can either be contracted to keep 3connectivity up to parallel pairs or deleted to keep 3-connectivity up to series pairs. If this element can be contracted so that $\operatorname{si}(M / e)$ is 3 -connected then contract $e$. Note that when we contract $e$ none of $r, g, x, y$ becomes parallel to any other of $r, g, x, y$. Therefore assume that $\operatorname{si}(M / e)$ is not 3-connected. This means that we can assume that $e$ is in the guts of a 3-separation. Consider $M \backslash e$. This is 3 -connected up to series pairs. If neither $\{g, x\}$ or $\{r, y\}$ is a series pair then the result follows by the claims above. Therefore without loss of generality assume that 
$\{g, x\}$ is a series pair in $M \backslash e$. This means that $\{g, x, e\}$ must be a triad, It is a well known theorem and can be found in [3] that if $e$ is in the guts of a 3-separation and $\{g, x, e\}$ form a triad then $x$ and $g$ are in different sides of the 3-separation. Consider $M / e$; the result follows from the claims above.

We have shown that when $M$ is not 3-connected then $M$ must be one of the matroids described in the statement of the lemma. We have also shown that $r(M) \leq 3$. Consider $M$. An element of $M$ can be contracted unless

1. it is in the guts of a 3-separation with $g, x$ on one side and $r, y$ on the other,

2. it is in a triangle with $x, g$,

3. it is in a triangle with $r, y$, or

4. it is in a triangle with $x, y$.

Since we are assuming that $M$ contains no 3-separations with $g, x$ on one side and $r, y$ on the other, $M$ must be one of the following matroids:
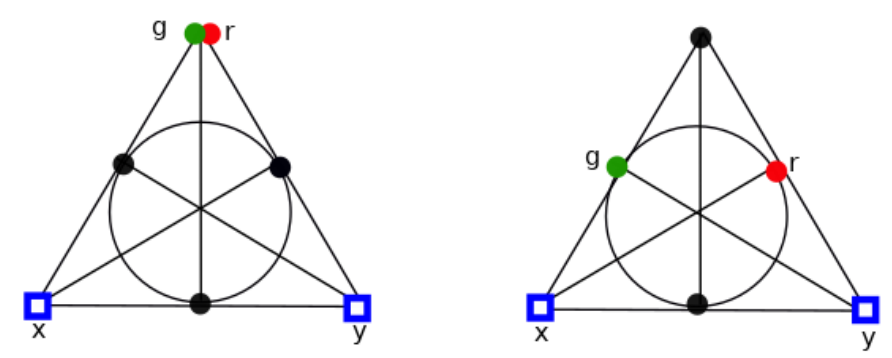

\subsection{Useful Lemmas For Reducing Petals Containing Representatives of a Lot of Blocking Elements}

In this section we prove the following theorem.

Theorem 8.4.1. There is a function 8 8.4.1 such that the following holds. Suppose $M$ is a binary matroid with a coindependent set $X$ such that is such that $M \backslash X$ has a maximal swirl-like pseudo-flower $F$, every 3-separation of $M \backslash X$ displayed 
by $F$ is blocked by an element of $X$ and $|X| \geq 8$ 8.4.1 $(t)$. Suppose $F$ has a petal $P$ containing a representative of every $x \in X$. Then either $M$ has a rank-t spike, $M\left(K_{3, t}\right)$ or $M^{*}\left(K_{3, t}\right)$-minor, or there is a minor $M^{\prime}$ of $M$ with coindependent set $X^{\prime}=E\left(M^{\prime}\right) \cap X$ such that the following hold.

I) $M^{\prime} \backslash X^{\prime}$ has a maximal swirl-like pseudo-flower $F^{\prime}$ of order $t$,

II) every 3-separation of $M^{\prime} \backslash X^{\prime}$ displayed by $F^{\prime}$ is blocked by an element of $X^{\prime}$ and $\left|X^{\prime}\right| \geq t$

III) $F^{\prime}$ has a petal $P$ containing a representative of every $x \in X^{\prime}$,

IV) If $P$ is a joint-based 2-petal then either

ii) The elements of $P$ form $a(t+1)$-element circuit with the joint of $P$ and all elements of $P-J(P)$ are the shadow of a unique $x \in X^{\prime}$, or

iiii) the elements of $P$ form a triangle with the joint of $P$ and one element in $P-J(P)$ is the shadow of all $x \in X^{\prime}$.

V) If $P$ is a rim-based 2-petal then either

(a) The elements of $P$ form a t-element circuit $C$ with the basepoint of $P$ parallel to some element of $C$ and all elements in $C$ the shadow of a unique $x \in X^{\prime}$, or

(b) $P=\{e\}$ and $e$ is parallel to the basepoint, $b$, of $P$ and for all $x \in X^{\prime}$, $F(x)$ contains $b$.

VI) If $P$ is a 3-petal then either

(a) The elements of $P$ are such that $W=M^{\prime} \mid P \cong M\left(\mathscr{W}_{t+2}\right)$ with the joints of $P$ adjacent to two joints of $W$, and every joint of $W$ that is not a basepoint of $P$ is the shadow of a unique $x \in X^{\prime}$, or

(b) The elements of $P$ are such that $W=M^{\prime} \mid P \cong M\left(\mathscr{W}_{t+2}\right)$ with the joints of $P$ adjacent to two rim elements of $W$, and every joint of $W$ that is not a basepoint of $P$ is the shadow of a unique $x \in X^{\prime}$, or

(c) The elements of $P$ are such that $W=M^{\prime} \mid P \cong M\left(\mathscr{W}_{t+2}\right)$ with the joints of $P$ such that one is parallel to a rim element of $W$ and one is parallel to a joint of $W$, and every joint of $W$ that is not a basepoint of $P$ is the shadow of a unique $x \in X^{\prime}$, or 
(d) $|P|=1$ and this element $e$ is parallel to the basepoint, $b$, of $P$ and for all $x \in X^{\prime}, F(x)$ contains $b$.

Consider a binary matroid $M$ and some fixed closed set $A \subseteq E(M)$. Every element $e$ of $E(M)-A$ is assigned some value $v_{M}(e)$ where $v_{M}(e) \in \mathbb{Z}_{\geq 0}$. The value of $M$, denoted $v_{M}$ is a non-negative integer. The value behaves as follows under contraction.

I If $z \in E(M)-A$ then the following hold.

i) The value of $M / z$ is $v_{M}-v_{M}(z)-v_{M}(B)$ where $B$ is the set of all elements in the closure of $A$ in $M / z$,

ii) the value of an element $e$ of $M / z$ is such that $v_{M / z}(e) \geq v_{M}(e)$ unless $e \in \mathrm{cl}_{M / z}(A)$ in which case $v_{M / z}=0$,

II if two elements $e$ and $f$ become parallel in $M / z$ then replace these elements by a new element $g$ such that $v_{M / z}(g)=v_{M / z}(e)+v_{M / z}(f)$.

To give this some context, consider a flower, $F$, in a matroid $M$ and a nonguts petal, $P$, of this flower containing representatives of blocking elements $X=x_{1}, \ldots, x_{n}$. For every $e \in P$ assign a number to $e$, that number being the number of elements of $X$ that do not contain a non-guts representative in $P-e$. These values behave in the way described above when we take minors of $M$. The closed set $A$ corresponds to the guts petal of $F$.

We omit the routine proof of the following lemma.

Lemma 8.4.2. Let $M$ be a matroid and let $p \in E(M)$ be such that $M \backslash p$ is connected. Then for all $e \in E(M \backslash p)$ either

i) $\{e, p\}$ is a parallel class in $M$,

ii) $M \backslash$ e and $M \backslash\{e, p\}$ are connected, or

iii) $M / e$ and $M \backslash p /$ e are connected.

Proof. Since $M \backslash p$ is connected either $M \backslash p / e$ or $M \backslash p \backslash e$ is connected. Suppose that $M \backslash p / e$ is connected, and suppose $M / e$ is not connected. This means that $e$ is the the guts of a 2-separation $(A, B)$ of $M$. However $e$ is not in the guts of 
a 2-separation in $M \backslash e$. Since $M$ and $M \backslash e$ are connected it follows that $\{e, p\}$ is a series class. Suppose $M \backslash\{e, p\}$ is connected and $M \backslash e$ is not connected. Then $e$ must be in the coguts of some 2-separation $(A, B)$ of $M$. Without loss of generality say $p \in A$. We know $\lambda_{M}(A)=1$ and $\lambda_{M \backslash e}(A)=0$, and $e$ is in the coclosure of $A$ and the coclosure of $B$. Since $M \backslash p$ is connected and $p$ is not in a circuit containing any elements of $B-e$, and $p$ is not in a circuit with any elements of $A-e$. Therefore every circuit containing $p$ must contain $e$, but then $e \in \operatorname{cl}(A)$, if $e \notin \mathrm{cl}(B)$ then this is a contradiction, and if $e \in \operatorname{cl}(B)$, then $M / e$ is not connected, again a contradiction.

Lemma 8.4.3. There is a function 8 8.4.3 such that the following holds. Let $M$ be a matroid and $j \in E(M)$ be such that $M$ and $M / j$ are connected. Suppose the

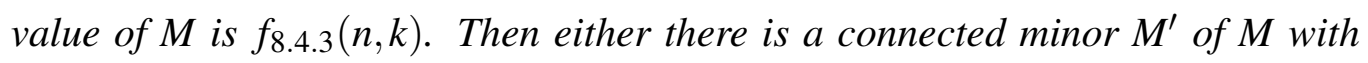
$\left|M^{\prime}\right|=n+1$ such that $M^{\prime} / j$ is connected and all elements of $E\left(M^{\prime}\right)-j$ have non-zero value, or there is a minor $M^{\prime \prime}$ of $M$ that is a triangle containing $j$, and $E\left(M^{\prime \prime}\right)-j$ contains an element with value at least $k$.

Proof. Let $v_{M}=n k$. Consider $e \in(E(M)-j)$ with $v_{M}(e)=0$. By Lemma 8.4.2 there is a connected minor $M_{1}$ of $M$ obtained by either deleting or contracting $e$ unless $\{e, j\}$ is a series class. Thus we can find a minor $M_{1}$ of $M$ such that $M_{1}$ and $M_{1} \backslash\{j\}$ are connected and $M_{1}$ contains at most one element $e \in E\left(M_{1}\right)-j$ with $v_{M_{1}}(e)=0$. Moreover, if $v_{M_{1}}(e)=0$ then $\{j, e\}$ is a series class. If $\{j, e\}$ is a maximal series class then contract $e$ to get a minor $M_{2}$ of $M_{1}$ with $v(e) \geq 1$ for all $e \neq j$, and $v_{M_{2}}=v_{M}$. Thus $M_{2}$ is a connected matroid such that $M_{2} / j$ is connected, $V_{M_{2}}=v_{M}$ and every element in $E\left(M_{2}\right)-j$ has value at least one.

If $\left|E\left(M_{2}\right)\right| \geq n+1$, then there are at least $n$ elements of $M^{\prime}$ with non-zero value. Therefore assume that $\left|E\left(M_{2}\right)\right| \leq n+1$. If there is an element $e$ of $M_{2}$ with $v_{M_{2}}(e) \geq k$ then contract all element of $M_{2}$ not in the closure of $\{e, j\}$ and the result follows. Suppose all $e \in\left(E\left(M_{2}\right)-j\right)$ have $v_{M_{2}}(e) \leq k$ and $v_{M_{2}}(e) \geq 1$. Contract some $e$. Then $v_{M_{2} / e} \geq v_{M}-k$. If some $f$ in $M_{2} / e$ has $v_{M_{2} / e} \geq k$ then contract all elements not on a line with $f$ and $j$, otherwise contract some $f$ in $M^{\prime} / e$ to get a minor of $M_{2} / e$ with $v_{M_{2} / e / f} \geq v_{M}-2 k$. Clearly we can continue in this way to find a minor $M_{a}$ of $M$ with an element $e$ with $v_{M_{a}} \geq k$ or a minor $M_{b}$ of $M$ that is a triangle containing $j$ and $v\left(M_{b}\right) \geq v_{M}-(n-1)(k-1) \geq n$.

Recall that if $M$ is a large connected matroid then $M$ either contains a large circuit or a large cocircuit. 
The routine proof of the following lemma is left to the reader.

Lemma 8.4.4. Let $M$ be a connected matroid containing an n-element circuit $C$. Then, for any $e \in(E(M)-C)$, there is a minor of $M$ such that: $e \in \operatorname{cl}(C)$ and there is a partition $(A, B)$ of $C$ such that either $A \cup e$ or $B \cup e$ is a circuit with at least $\frac{n}{2}$ elements.

By duality we get the following lemma.

Lemma 8.4.5. Let $M$ be a connected matroid containing an n-element cocircuit $C^{*}$. Then, for any $e \in\left(E(M)-C^{*}\right)$, there is a minor $M^{\prime}$ of $M$ on $C^{*} \cup$ e with $e \in \mathrm{cl}^{*}\left(C^{*}\right)$ in which $A, B$ partitions $C^{*}$ and either $A \cup e$ or $B \cup e$ is a cocircuit with at least $\frac{n}{2}$ elements. Moreover, $\mathrm{si}\left(M^{\prime}\right)$ is a triangle and there is no element parallel to $e$ in $M^{\prime}$.

Putting this together we have the following:

Lemma 8.4.6. Let $M$ be a binary matroid with coindependent set $X$ such that the following hold.

i) $|X|=m$,

ii) $M \backslash X$ has a swirl-like pseudo-flower $F=\left(P_{1}, \ldots, P_{m}\right)$, and

iii) $X$ is a minimal set of blocking elements for the displayed 3-separations of $M \backslash X$

. If $P_{i}$ is a joint-based 2-petal of $F$ that contains representatives of at least every element of $X$ and $m \geq \sqrt{8.4 .3}(\sqrt{2.4 .1}(2 t))$ blocking elements, then there is a minor $M^{\prime}$ of $M$ such that the following holds:

i) $M^{\prime} \backslash\left(E\left(M^{\prime}\right) \cap X\right)$ has a swirl-like pseudo-flower $F^{\prime}=\left(P_{1}, \ldots, P_{i-1}, P_{i}^{\prime}, P_{i+1}, \ldots, P_{t}\right)$,

ii) $X^{\prime}=E\left(M^{\prime} \cap X\right)$ blocks all 3-separations of $M^{\prime} \backslash X^{\prime}$ displayed by $F$,

iii) $M^{\prime} \mid P_{i}^{\prime}$ contains representatives of every element of $X^{\prime}$, and either the representatives of these t elements are distinct and form a circuit with $J\left(P_{i}^{\prime}\right)$, or there is one element in $P_{i}^{\prime}$ that is a representative of $t$ blocking elements and this element is in a triangle with $J\left(P_{i}^{\prime}\right)$, 
iv) all elements of $P^{\prime}$ are either a representative of a blocking element of $P$, are $j$, or are in a triangle with $j$ and an element that is a representative of a blocking element.

Proof. Let $N=M \mid P_{i}$. Consider the value function described earlier. Let $v_{M / P_{i}}=$ $m$ and to every $e \in P_{i}$ assign to it a value equal to the number of unblocked 3separations in $F$ in $M \backslash e$. It is easy to see that this assignment of values behaves as required. By Lemma 8.4.3 there is a minor $N_{1}$ of $N$ with at least $\int[2.4 .1(2 t)+1$ elements such that $N_{1}$ is connected and all elements of $N_{1}$ have non-zero value or there is a minor $N_{2}$ of $N$ that is a triangle including $j$ and an element with value at least $\int[2.4 .1(2 t)$.

If there is a minor $N_{1}$ of $N$ with at least $\int_{2.4 .1}(2 t)+1$ elements such that $N_{1}$ is connected and all elements of $N_{1}$ have non-zero value then by Lemma 2.4.1 there is a minor of $N$ containing a circuit with at least $2 t$ elements or a cocircuit with at least $2 t$ elements all of which have non-zero value. Therefore by Lemma 8.4.4 and its dual there is a minor, $N^{\prime}$, of $N$ with value at least $t$ that is either a circuit or a cocircuit containing $e$.

The result then follows easily.

We now look at rim-based 2-petals. This case is very similar to the dual of the case above. However, very close is not close enough so we have the following:

Lemma 8.4.7. Let $M$ be a matroid and $r \in M$ such that $M$ and $M \backslash r$ are connected. Suppose the value of $M$ is at least $\sqrt{8.4 .3}(n, k)$. There is a minor $M^{\prime}$ of $M$ such that $M^{\prime}$ and $M^{\prime} \backslash r$ are both connected and either

i) $M^{\prime}$ contains at least $n$ elements of non-zero value, or

ii) $M^{\prime}$ is a triangle with value at least $k$.

Proof. By Lemma 8.4.2 we may assume that every element in $M$ has non-zero value or $M$ is a triangle. If $M$ is a triangle then $i i)$ follows. Therefore assume that every element in $E(M)-r$ has non-zero value. If $M$ has at least $n$ elements then the result follows. Therefore assume that $M$ has fewer than $n$ elements. The remainder of the proof is inductive. Suppose $M^{\prime}$ is a connected minor of $M$ that has 3 elements, one of which is $r$, and value at least $n$. Then $i i)$ holds. Let $M^{\prime}$ be a minor of $M$ with value at least $v_{M}-a(n-1)$ for some $a \leq(n-2)$, with the 
property that both $M^{\prime}$ and $M^{\prime} \backslash r$ are connected. Suppose that there is an element of $M$ with value at least $n$, then there is a minor $M^{\prime}$ of $M$ that is a triangle containing $r$ with value at least $n$. Suppose there is no element of $M$ with value at least $n$. Then consider some element $e \in E\left(M^{\prime}\right)$ with $n>v(e) \geq 1$. We may remove $e$ in such a way that there is a minor $M^{\prime \prime}$ of $M^{\prime}$ with the property that $M^{\prime \prime}$ are $M^{\prime \prime} \backslash r$ are both connected. Note that $v_{M^{\prime \prime}} \geq v_{M^{\prime}}-(n-1)$. Since $M^{\prime \prime}$ has fewer than $n$ elements the result follows.

The following proof is courtesy of James Oxley.

Lemma 8.4.8. Let $M$ be a binary matroid with an element $r \in E(M)$ such that $M$ and $M \backslash r$ are connected, and $|E(M)|=\int_{2.4 .1}(2 n)+1$. Then $M$ has a minor $M^{\prime}$ containing $r$ with the property that $M^{\prime} \backslash r$ is a circuit $C$ containing $n$ elements and $r$ is parallel to some element of $C$, or $M^{\prime}$ is a parallel class containing $r$ of size at least $n$.

Proof. By Lemma 2.4.1, $M \backslash r$ has a set $X$ with at least $2 n$ elements such that $X$ is a circuit or a cocircuit of $M \backslash r$. If $M$ has a cocircuit containing $r$ and having at least $n+1$ elements, then the lemma holds as the contraction of $M$ onto the elements of this cocircuit is a parallel class. It follows that if $X$ is a cocircuit of $M \backslash r$, then $X$ is a cocircuit of $M$. Thus $X$ is a circuit or a cocircuit of $M$, so $M$ has a minor $N$ with ground set $X$ such that $N$ is a circuit or a cocircuit with at least $2 n$ elements. By Tuttes Linking Theorem, $M$ has a connected minor $N_{1}$ with ground set $X \cup r$ such that $N_{1} \mid X=N$. Suppose $X$ is a circuit. Then $r$ is in the closure of $X$ in $N_{1}$. The dual of $N_{1}$

is a 3-point line with $e$ as a rank-one flat and with two other rank-one flats, $X_{1}$ and $X_{2}$, whose union is $X$. Assume $\left|X_{1}\right| \geq\left|X_{2}\right|$. Take $x$ in $X_{2}$. Then $N_{1} /\left(X_{2}-x\right)$ consists of a circuit with ground set $X_{1} \cup x$ and with the element $r$ in parallel to $x$. If, instead, $X$ is a cocircuit, then, since $X \cup r$ is not a cocircuit of $N_{1}$, we see that $N_{1}$ has rank two and has $\{e\}$ as a hyperplane. It follows that $N_{1}$ has a cocircuit containing $r$ and having at least $n+1$ elements.

The proof of the following lemma is similar to that of Lemma 8.4.6 and is omitted.

Lemma 8.4.9. If $P_{i}$ is a rim-based 2-petal of $F$ that contains a representative of at least $\int_{8.4 .3}\left(f_{2.4 .1}(2 n)\right)$ blocking elements, then there is a minor $M^{\prime}$ of $M$ such that $M^{\prime} \backslash X$ has a flower $F^{\prime}=\left(P_{1}, \ldots, P_{i-1}, P_{i}^{\prime}, P_{i+1}, \ldots, P_{n}\right)$ and $M^{\prime} \mid P_{i}^{\prime}$ contains representatives of at least $n$ blocking elements $\left\{x_{1}, \ldots, x_{n}\right\}$. Moreover, the representatives 
of $\left\{x_{1}, \ldots, x_{n}\right\}$ in $P_{i}^{\prime}$ are either distinct and form a circuit with an element of this circuit parallel to $j$, or there is one element in $P_{i}^{\prime}$ that is a representative of $n$ blocking elements and this element is parallel to $j$. Moreover, all elements of $P_{i}^{\prime}$ are a representative of some blocking element.

Now consider 3-petals.

Lemma 8.4.10. Let $M$ be a connected binary matroid containing two elements $j_{1}$ and $j_{2}$ with the property that for any e, $f$ with $v(e), v(f) \neq 0$ there is no proper 2-separation $(A, B)$ with $j_{1}, e \in A$ and $e \notin \operatorname{cl}(B)$ and $j_{2}, f \in B$ and $f \notin \operatorname{cl}(A)$. Then there is a connected minor $M^{\prime}$ of $M$, with $v_{M^{\prime}}=v_{M}$, containing $j_{1}$ and $j_{2}$ such that, for any e, $f \in\left(E\left(M^{\prime}\right)-\left\{j_{1}, j_{2}\right\}\right)$ with $v(e), v(f) \neq 0$, there is no proper 2separation $(A, B)$ of $M^{\prime}$ with $j_{1}, e \in A$ and $e \notin \operatorname{cl}(B)$ and $j_{2}, f \in B$ and $f \notin \operatorname{cl}(A)$, and every $g \in\left(E\left(M^{\prime}\right)-\left\{j_{1}, j_{2}\right\}\right)$ with $v_{M^{\prime}}(g)=0$ is on the guts of a 3-separation $(C, D)$ of $M^{\prime}$ with $j_{1}, e \in C$ and $e \notin \operatorname{cl}(D)$ and $j_{2}, f \in D$ and $f \notin \operatorname{cl}(C)$ for $e, f$ with $v(e), v(f) \neq 0$.

Proof. Note that any element $g \in E(M)$ can either be deleted to keep connectivity or contracted to keep connectivity. Therefore we may remove any $g \in E(M)$ with $v(g)=0$ unless this removal results in a 2-separation $(A, B)$ with $j_{1}, e \in A$ and $e \notin \operatorname{cl}(B)$ and $j_{2}, f \in B$ and $f \notin \operatorname{cl}(A)$ for some $e, f$ with $v(e), v(f) \neq 0$. Therefore we can remove any $g \in E(M)$ unless $f$ is on the guts of a 3-separation $(A, B)$ with $j_{1}, e \in A$ and $e \notin \operatorname{cl}(B)$ and $j_{2}, f \in B$ and $f \notin \operatorname{cl}(A)$ for some $e, f$ with $v(e), v(f) \neq$ 0 .

Lemma 8.4.11. There is some 8.4.11 such that the following holds. Let $M$ be a connected matroid with $v_{M}=\sqrt{8.4 .11}(n)$. Suppose there are elements $j_{1}, j_{2} \in E(M)$ such that $v\left(j_{1}\right)=v\left(j_{2}\right)=0$ and suppose $M$ is such that the following holds:

I) for any e, $f \in E\left(M^{\prime}\right)$ with $v(e), v(f) \neq 0$ there is no 2-separation $(A, B)$ of $M^{\prime}$ such that both the following holds.

- $j_{1}, e \in A$ and $e \notin \mathrm{cl}(B)$, and

- $j_{2}, f \in B$ and $f \notin \operatorname{cl}(A)$.

II) Every $g \in\left(E\left(M^{\prime}\right)-\left\{j_{1}, j_{2}\right\}\right)$ with $v_{M^{\prime}}(g)=0$ is on the guts of a 3-separation $(C, D)$ of $M^{\prime}$ with $j_{1}, e \in C$ and $e \notin \operatorname{cl}(D)$, and $j_{2}, f \in D$ and $f \notin \operatorname{cl}(C)$ for $e, f$ with $v(e), v(f) \neq 0$. 
Then $M$ has a minor $M^{\prime}$ containing $j_{1}$ and $j_{2}$ such that either

i) $M^{\prime}$ is 3-connected and $v_{M}^{\prime} \geq n$,

ii) $v_{M^{\prime}} \geq n$ and $j_{1}, j_{2} \notin \operatorname{cl}\left(E\left(M^{\prime}\right)-\left\{j_{1}, j_{2}\right\}\right)$ but $r_{1} \in\left\langle E\left(M^{\prime}\right)-\left\{j_{1}, j_{2}\right\}\right\rangle$ for $r_{1} \in\left\langle j_{1}, j_{2}\right\rangle$, or

iii) $v_{M^{\prime}} \geq n$ and, there is an element $r_{1} \in E\left(M^{\prime}\right)$ such that $j_{1}, r_{1}, j_{2}$ is a triangle and either $j_{1} \in \operatorname{cl}\left(E\left(M^{\prime}\right)-\left\{j_{1}, j_{2}, r_{1}\right\}\right)$ or $j_{2} \in \operatorname{cl}\left(E\left(M^{\prime}\right)-\left\{j_{1}, j_{2}, r_{1}\right\}\right)$ and not both.

Proof. Let $\sqrt{8.4 .11}(n)=n^{2}$. Suppose that $M$ is not 3-connected. For any $Y \subseteq E(M)$ let $v_{M}(Y)$ be the sum of the values of the elements of $Y$. Suppose $(A, B)$ is a 2separation of $M$ with $v_{M}(A) \geq m$ and $j_{1}, j_{2} \in B$. By Lemma 2.1.16, and a case analysis similar to that of Lemma 8.3.4, there is a minor $M^{\prime}$ of $M$ satisfying either i) or $i$ ), with $v_{M}^{\prime} \geq m$. Now assume that there is no 3-connected set $A$ of $M$ with $j_{1}, j_{2} \in A$ and $v_{M}(A) \geq n$. Suppose there are at least $n$ 2-separations, $\left(A_{i}, B_{i}\right)$ for $i \in\{1, \ldots, n\}$, with $j_{1}, j_{2} \in A$ and an element with non-zero value in $B$. Then it is routine to chack that there is a minor of $M$ that is 3 -connected and has value at least $n$.

Therefore we may assume that every 3 -separation $\left(A_{i}, B_{i}\right)$ that such that $j_{1}, j_{2} \in A_{i}$ has $v\left(B_{i}\right)<n$ and there are fewer than $n$ such 3-separations. This means that there is a 3-connected minor $M^{\prime}$ of $M$ with $v_{M^{\prime}} \geq n$ and $j_{1}, j_{2} \in M^{\prime}$.

Cases ii) and iii) from above reduce to the 2-petal case. Thus we consider reducing petals where $M$ is 3 -connected and $v_{M} \geq n$.

First we need the following routine lemma.

Lemma 8.4.12. Let $M$ be a matroid with 3-separation $(A, B)$ that contains an element $e \in E(M)$ such that $e \in \operatorname{cl}(A) \cap \operatorname{cl}(B)$. If $a_{1}, a_{2}, a_{3} \in(A-e)$ and $b_{1}, b_{2}, b_{3} \in$ $(B-e)$, then for any 3-connected minor $M^{\prime}$ of $M$ containing $a_{1}, a_{2}, a_{3}, e, b_{1}, b_{2}, b_{3}$, the element $e$ is in the guts of a 3-separation $\left(A^{\prime}, B^{\prime}\right)$ with $a_{1}, a_{2}, a_{3} \in A^{\prime}$ and $b_{1}, b_{2}, b_{3} \in B^{\prime}$.

We also need the following theorem from [4]. 
Theorem 8.4.13. There is a function 8 8.4.13 such that the following holds. Suppose $M$ is a 3-connected binary matroid with $|E(M)| \geq f_{8.4 .13}(n)$ and $\{x, y\} \subseteq E(M)$. There there is a minor of $M$ using $x$ and $y$ that is isomorphic to $M\left(\mathscr{W}_{n}\right)$, a rank-n spike, $M\left(K_{3, n}\right)$ or $M^{*}\left(K_{3, n}\right)$.

Lemma 8.4.14. Let $M$ be a 3-connected matroid with $v_{M}=\sqrt{8.4 .14}(n)$. Suppose that there are elements $j_{1}, j_{2} \in E(M)$ such that $v\left(j_{1}\right)=v\left(j_{2}\right)=0$ and every $g \in$ $E(M)-\left\{j_{1}, j_{2}\right\}$ with $v_{M^{\prime}}(g)=0$ is on the guts of a 3-separation $(C, D)$ of $M^{\prime}$ such that for some e, $f \in E\left(M^{\prime}\right)$ with $v(e), v(f) \neq 0$, the elements $j_{1}, e \in A$ and $e \notin \operatorname{cl}(B)$ and $j_{2}, f \in B$ and $f \notin \operatorname{cl}(A)$ for $e, f$. Then there is a minor of $M$ containing $j_{1}$ and $j_{2}$ that is isomorphic to one of the following.

i) $M\left(K_{3, n}\right)$

ii) $M^{*}\left(K_{3, n}\right)$,

iii) a rank-n spike,

iv) an $n+2$-spoke wheel with value at least $n$ in which every rim element not in $\left\{j_{1}, j_{2}\right\}$ is assigned a non-zero value, or

v) $M\left(K_{4}\right)$ with value at least $n$.

Proof. If $M$ has at least $\sqrt{8.4 .13}(n+8)$ elements then $M$ has either an $M\left(K_{3, n}\right)$ minor, an $M^{*}\left(K_{3, n}\right)$-minor, a rank- $n$ spike or an $(n+8)$-spoke wheel as a minor. We must check that in the case where $M$ has an $(n+8)$-spoke wheel as a minor, $M$ has an $n$-spoke wheel as a minor with value at least $n$. Suppose $M$ had an $n+8$ spoke wheel as a minor. For every $e_{i}$ with $v\left(e_{i}\right)=0$ there is a 3 -separation $\left(A_{i}, B_{i}\right)$ with $j_{1}, a_{i} \in A_{i}$ for some $a_{i}$ with $v\left(a_{i}\right) \neq 0$ and $j_{2}, b_{i} \in B_{i}$ for some $b_{i}$ with $v\left(b_{i}\right) \neq 0$. Consider two crossing 3-separations of this form and without loss of generality let them be $\left(A_{1}, B_{1}\right)$ and $\left(A_{2}, B_{2}\right)$. Without loss of generality let $a_{i} \in A_{1} \cap A_{2}$. By uncrossing $A_{1} \cap A_{2}$ is a 3-separation and since $\left|A_{1} \cap A_{2}\right| \geq 3$ this is a vertical 3separation with $a_{i}$ in the guts. In this way we can uncross all 3 -separations to get a nested sequence of 3-separations and thus a natural ordering on the elements with zero value; that being an element $e$ with value 0 is greater than an element $f$ with value 0 if $\left(A_{e}, B_{e}\right)$ is a separation of $M$ with $e$ in the guts that does not cross $\left(A_{f}, B_{f}\right)$, a separation of $M$ with $f$ in the guts, and $A_{f} \subseteq A_{e}$. Since we have a sequence of non-crossing 3 -separations this ordering is well defined. Assign the 
elements $e$ of $M$ with $v(e)=0$ labels from $e_{1}, \ldots, e_{m}$ where the subscripts reflect the ordering on these elements. Consider a wheel minor of $M$ and suppose it contains more than eight elements with zero value as rim elements. One of these, call it $e$, must have the property that there are at least four rim elements less $e$ and at least four elements greater than $e$. This $e$ is then in the guts of a 3-separation which contradicts Lemma ??. Therefore all but eight rim elements must have value at least one, and the result follows.

Now suppose $M$ has fewer than $\sqrt{8.4 .13}(n+8)$ elements. Suppose that some element $e$ of $M$ has value at least $n$. Then, by Lemma 2.1.16, $M$ had an $M\left(K_{4}\right)$-minor using $e, j_{1}, j_{2}$ with value at least $n$.

Suppose that there is no element with value at least $n$. We may contract any element $e$ with value less than $n$ to find a matroid $M^{\prime}$ with $v_{M^{\prime}} \geq v_{M}-n$ unless $e$ is on the guts of a 3-separation that puts some element with non-zero value and $j_{1}$ on one side and some other element with non-zero value and $j_{2}$ on the other side. Consider all elements $e \subseteq E(M)$ of this form. An uncrossing argument similar to that above shows that we have an ordering on these elements. Consider the first element in this ordering. There is a separation $(A, B)$ with $j_{1}, f \in A$ where $j_{1}$ has non-zero value. We may then contract $f$ and, as this may result in a 2-separation, find a minor of $M / f$ that is 3-connected, contains $j_{1}$ and $j_{2}$ and has value at least $v_{M}-n$. We can repeat this process until a 3 -connected minor of $M$ containing $j_{1}$ and $j_{2}$ either has an element with high value or has a rank-3 3-connected minor containing $j_{1}$ and $j_{2}$ with value at least $\left.v_{M}-(\sqrt[8.4 .13]{ }(n)-1)\right)(n-1)$.

The proof of the following lemma is similar to that of Lemma 8.4.6.

Lemma 8.4.15. There is a function 8 such that the following holds. Suppose $M$ is a matroid with a coindependent set $X$ such that is such that $M \backslash X$ has a maximal swirl-like pseudo-flower $F$, every 3-separation of $M \backslash X$ displayed by $F$ is blocked by an element of $X$ and $|X| \geq \sqrt{8.4 .14}(t)$. Suppose $F$ has a petal $P$ containing a representative of every $x \in X$. Then either $M$ has a rank-t spike, $M\left(K_{3, t}\right)$ or $M^{*}\left(K_{3, t}\right)$-minor, or there is a minor $M^{\prime}$ of $M$ with coindependent set $X^{\prime}=E\left(M^{\prime}\right) \cap X$ such that the following holds.

I) $M^{\prime} \backslash X^{\prime}$ has a maximal swirl-like pseudo-flower $F^{\prime}$ of order $t$,

II) every 3-separation of $M^{\prime} \backslash X^{\prime}$ displayed by $F^{\prime}$ is blocked by an element of $X^{\prime}$ and $\left|X^{\prime}\right| \geq t$, 
III) $F^{\prime}$ has a petal $P$ containing a representative of every $x \in X^{\prime}$,

IV) If $P$ is a 3-petal containing representative of at least $\sqrt{8.4 .15}(t)$ elements of $X$ then either.

(a) The elements of $P$ are such that $W=M^{\prime} \mid P \cong M\left(\mathscr{W}_{t+2}\right)$ with the joints of $P$ adjacent to two joints of $W$, and every joint of $W$ that is not a basepoint of $P$ is the shadow of a unique $x \in X^{\prime}$, or

(b) The elements of $P$ are such that $W=M^{\prime} \mid P \cong M\left(\mathscr{W}_{t+2}\right)$ with the joints of $P$ adjacent to two rim elements of $W$, and every joint of $W$ that is not a basepoint of $P$ is the shadow of a unique $x \in X^{\prime}$, or

(c) The elements of $P$ are such that $W=M^{\prime} \mid P \cong M\left(\mathscr{W}_{t+2}\right)$ with the joints of $P$ such that one is parallel to a rim element of $W$ and one is parallel to a joint of $W$, and every joint of $W$ that is not a basepoint of $P$ is the shadow of a unique $x \in X^{\prime}$, or

(d) $|P|=1$ and this element e is parallel to the basepoint, $b$, of $P$ and for all $x \in X^{\prime}, F(x)$ contains $b$.

Proof. Let $N=M \mid P_{i}$. Consider the value function described earlier. Let $v_{M / P_{i}}=$ $m$ and to every $e \in P_{i}$ assign to it a value equal to the number of unblocked 3separations in $F$ in $M \backslash e$. It is easy to see that this assignment of values behaves as required. The lemma then follows easily from Lemma 8.4.14

The proof of Theorem 8.4 .1 is now routine and is left to the reader. 


\section{Chapter 9}

\section{Blocking Swirl-Like Pseudo-Flowers}

In this chapter we prove the following.

Theorem 9.0.1. There is a function $\sqrt{9.0 .1}$ such that for all $t \geq 5$ the following hold. If $M$ is a binary matroid with a coindependent set $X$ such that $M \backslash X$ has a maximal swirl-like pseudo-flower $F$ of order $n$ where $n \geq \sqrt{9.0 .1}(t)$, and every 3-separation of $M$ displayed by $F$ is blocked by an element of $X$, then $M$ has a minor isomorphic to one of the following:

i) a rank-t circular ladder,

ii) a rank-t Möbius ladder,

iii) a rank-t spike,

iv) a rank-t double wheel,

v) a rank-t non graphic double wheel,

vi) $N\left(K_{3, t}\right)$,

vii) $M\left(K_{4, t}\right)$,

viii) a rank-t clam.

This chapter splits into five main parts. One for when the swirl-like pseudo-flower is blocked by a single element, one when the crossing graph for the blocking elements contains a big star, one when it contains a big complete graph and one 
for when it contains a long path. The final section of this chapter brings all this together to give a proof of Theorem 9.0.1. Throughout this chapter we work under the hypotheses of Theorem 9.0.1. We restate these hypotheses below and introduce some local notation.

- $M$ is a binary matroid with coindependent set $X$ of $E(M)$ such that $M \backslash X$ has maximal swirl-like pseudo-flower $F=\left(P_{1}, \ldots, P_{n}\right)$ of order $m$,

- $\widetilde{M}$ denotes the matroid $M$ extended by the joints of $F$,

- $X$ is a minimal set of blocking elements for $F$ in $M$,

- $|X| \geq n^{\prime}$ for some $n^{\prime} \in \mathbb{Z}_{\geq 0}$,

- $J$ is the set of joints of $F$,

- $B$ is a basis for $\widetilde{M}$ containing the joints of $F$,

- $F(x)$ denotes the fundamental circuit of an element $x \in X$ with respect to $B$.

In a slight abuse of notation we use $\widetilde{M}^{\prime}$ when $M^{\prime}$ is a minor of $M$ to denote $M^{\prime}$ extended by the joints of $F$.

We may assume that there is no $x \in X$ such that $x$ is contained the closure of a clump of $F$ in $M \backslash X$. This is because the flower $M \backslash(X-x)$ has a maximal swirl-like pseudo-flower of order $n$ and we could consider this instead of $F$ in $M$. Therefore we can, without loss of generality, add the following hypothesis:

- Every $x \in X$ blocks some separation of $M$ displayed by $F$.

We are going to introduce yet another type of colouring and this time instead of colouring both joint and rim elements we colour just the joints.

Recall that $J\left(P_{i}\right)$ denotes the joints of petal $P_{i}$ of $F$.

Definition 9.0.2. The joints of $x \in X$ are the members of the set $J(x)=\{j \in J$ : there is some $P_{i}$ with $j \in J\left(P_{i}\right)$ and some element of $F(x) \in P_{i}$.

Further, recall that $\gamma: X \rightarrow C$ is a bijective function mapping members of $X$ to colours. This function does not really do anything but it can be helpful to consider colours rather than elements of $X$. 
We shall now colour the joints of $F$ in $M$.

Definition 9.0.3. Let $\mu_{J}: J \rightarrow \mathscr{P}(C)$ be such that $\mu(j)=\left\{c: \gamma^{-1}(c)\right.$ contains a representative in a petal $P_{i}$ with $\left.j \in J\left(P_{i}\right)\right\}$. We call $\mu$ the joint colouring of $F$ in $M$ with respect to $X$.

From now on, when we refer to a colouring we are referring to the joint colouring unless otherwise stated.

Definition 9.0.4. We say that two elements $x_{1}, x_{2} \in X$ are distinguishable by a set $J_{1}$ of joints in the joint colouring of $F$ in $M \backslash X$ with respect to $X$ if $J\left(x_{1}\right) \cap J_{1} \nsubseteq$ $J\left(x_{2}\right) \cap J_{1}$ and $J\left(x_{2}\right) \cap J_{1} \nsubseteq J\left(x_{1}\right) \cap J_{1}$.

We extend this in the natural way to talk about colours being distinguishable in a set of joints.

\section{A Note On Pictures}

In this chapter it helps to use pictures to illustrate certain features of $M$ and $F$. We will frequently use pictures when we are talking about joint colourings. When we do this the joints of $F$ are represented by circles and the colours of the circles represent colours in $\gamma(X)$. When two colours appear in (almost) the same place this means that the joint is coloured by multiple colours. Each colour represents a distinct element of $\gamma(X)$ and two colours are the same in a picture exactly when they represent the same colour in $\gamma(X)$. Ellipses are used to show that the pattern seen in the colours continues. We have more complicated pictures later on but explain these when they arise.

\subsection{Single Blocking Element}

Consider the case where a single blocking element contains representatives in at least $k$ petals.

Lemma 9.1.1. There is a function, $\sqrt{9.1 .1}$, such that the following holds. If at least $k \geq f_{9.1 .1}(t)$ displayed 3-separations of $M$ are blocked by some element $x$, then $M$ has a wheel minor with at least $t$ joints in which every displayed 3-separation is blocked by a single element, or M has a rank-t spike minor. 
Proof. Let $k \geq 2 t^{2}$. If $x$ contains representatives in at least $2 t$ clumps then it follows easily from Lemma 2.1 .16 that $M$ has a wheel minor with at least $2 t$ joints in which at least $t$ displayed 3-separations are blocked by $x$. Removing petals then gives a wheel minor with at least $t$ joints in which every displayed 3-separation is blocked by a single element. If $x$ contains representatives in fewer than $2 t$ clumps then at least one clump of $F$ contains at least $t$ petals each containing a representative of $x$. By removing all petals not in this clump and all but the petals containing a representative of $x$ in this clump we see that there is a minor $M^{\prime}$ of $M$ such that $M^{\prime} \backslash x$ has a $(1,0,0)$-flower $F^{\prime}$ and $x$ is not in the closure of a petal of $F^{\prime}$ and $x^{\prime} \in \operatorname{cl}\left(E\left(M^{\prime}\right)-x\right)$. By Lemma 2.1.16 it is easy to see that $M^{\prime}$ has a minor $M^{\prime \prime}$ of $M^{\prime}$ such that $M^{\prime \prime} \backslash x$ has a $(1,0,0)$-flower $F^{\prime \prime}$ with at least $t$ petals such that $x$ not in the closure of a petal of $F^{\prime \prime}$, that $x \in \operatorname{cl}\left(E\left(M^{\prime \prime}\right)-x\right)$, and that every petal $P$ of $F^{\prime \prime}$ is a series pair with basepoint the basepoint of the petal. It is then easy to see that $M^{\prime \prime}$ is a spike with cotip $x$, in other words that $M^{\prime \prime} / x$ is a spike.

Lemma 9.1.2. If $M$ is such that $M \backslash X$ is a rank-n wheel and there is some $x$ that blocks every 3-separation of $M \backslash X$ displayed by the canonical flower of $M \backslash X$, then $M$ has a rank- $(n-1)$ circular ladder as a minor.

Proof. $M$ is represented by

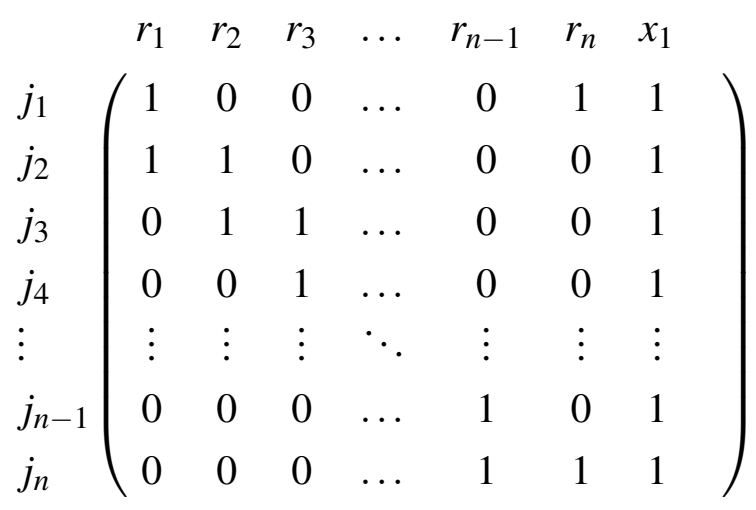


Pivot on $M_{j_{1}, x_{1}}$ to get

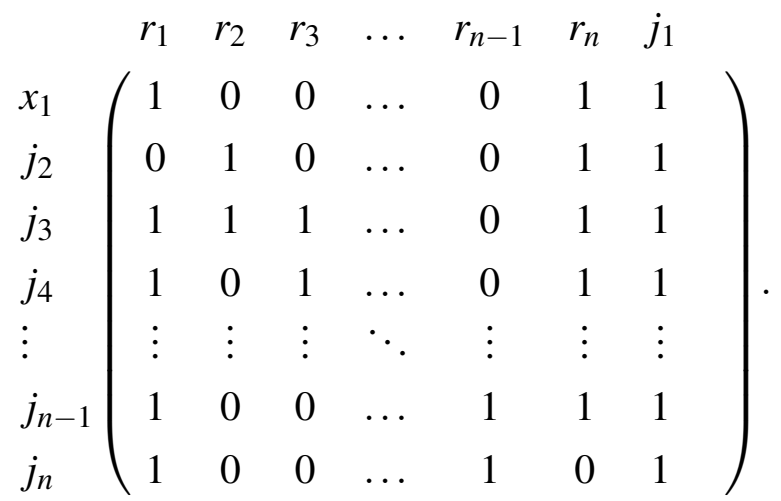

It is then easy to see that $M / x_{1}$ is a rank- $(n-1)$ triangular ladder and therefore $M$ has a rank- $(n-1)$ circular ladder as a minor.

Theorem 9.1.3. There is a function $\sqrt{9.1 .3}$ such that the following holds. Suppose $M$ is a binary matroid with coindependent set $X$ such that $M \backslash X$ has a swirl-like pseudo-flower $F$ and every displayed 3-separation in $M \backslash X$ is blocked by some $x \in X$. If some $x \in X$ blocks at least $k$ displayed 3-separations of $M \backslash X$ by $F$ and

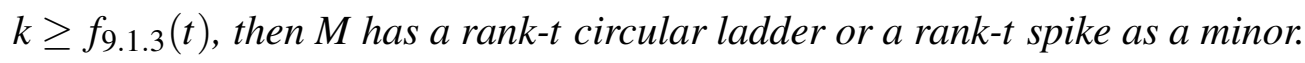

Proof. Let $f$ 9.1.3 $(t)=\int 9.1 .1(t+1)$. By Lemma 9.1.1 $M$ has a minor isomorphic to a rank- $(t+1)$ spike or a rank- $(t+1)$ wheel in which every vertical 3-separation is blocked by a single element. If $M$ has a rank- $(t+1)$ spike as a minor then the theorem follows. Suppose that $M$ has a rank $(t+1)$-wheel in which every vertical 3 -separation by a single element, as a minor. Then the theorem follow by Lemma 9.1.2.

\subsection{Stars}

In this section we prove the following theorem.

Theorem 9.2.1. There is a function $\sqrt{9.2 .1}$ such that for all $t \geq 5$ the following holds. If $M$ is a binary matroid with a coindependent set $X$ such that

I) $M \backslash X$ has a maximal swirl-like pseudo-flower of order $n$ where $n \geq \int_{9.0 .1}(t)$,

II) every 3-separation of $M$ displayed by $F$ is blocked by an element of $X$, 
III) the crossing graph of $X$ with respect to $F$ in $M$ is a star,

$I V)$ there is no $x \in X$ that contains a representative in $k$ or more petals,

then $M$ has minor isomorphic to one of the following:

i) a rank-t spike,

ii) a rank-t double wheel,

iii) a rank-t non graphic double wheel,

iv) $M^{*}\left(K_{3, t}\right)$.

Throughout this section we work under the hypotheses of Theorem 9.2.1. That is we add to our original hypotheses the following hypotheses.

- The crossing graph of $X$ with respect to $F$ in $M$ is a star, and

- no element of $X$ contains representatives in $k$ or more petals (where $k$ is large)

Clearly $|X|=n^{\prime} \geq \frac{n}{k}$.

We may, without loss of generality, restrict our attention to the set of blocking elements of $X$ that are distinguishable from the joint colouring of $F$ in $M \backslash X$ with respect to $X$. That is we may assume that if $x_{1}, x_{2} \in X$ then $J\left(x_{1}\right) \nsubseteq J\left(x_{2}\right)$ and $J\left(x_{2}\right) \nsubseteq J\left(x_{1}\right)$. Therefore we add the following hypothesis.

- For any pair $x_{1}, x_{2} \in X, J\left(x_{1}\right) \nsubseteq J\left(x_{2}\right)$ and $J\left(x_{2}\right) \nsubseteq J\left(x_{1}\right)$.

Lemma 9.2.2. There is a function $\sqrt{9.2 .2}$ such that the following holds. If $n \geq$ f9.2.2 $(t)$, then there is a minor $M^{\prime}$ of $M$ such that the following hold:

i) $M^{\prime} \backslash\left(E\left(M^{\prime}\right) \cap X\right)$ has a swirl-like pseudo-flower $F^{\prime} \subseteq F$,

ii) $E\left(M^{\prime}\right) \cap X=X^{\prime}$ is a coindependent set that is a minimal blocking set of $F^{\prime}$,

iii) $F^{\prime}$ has order at least $t$,

iv) $F^{\prime}$ is such that no proper petal contains a representative of more than one element of $X^{\prime}$, and 
v) the crossing graph of $X^{\prime}$ in $F^{\prime}$ with respect to $M$ is a star.

Proof. Consider $n \geq t+k^{2}$. Let $x \in X$ be the element that crosses all other elements of $X$. This is the only element of $X$ that contains representatives in proper petals that contain representatives of an element of $X-x$. Therefore at most $k$ colours are in a petal containing representatives of more than one element. Delete any element $x^{\prime} \in X$ except $\gamma(x)$ that contains representatives in a petal containing a representative of $x$. Let these elements be $\left\{x_{1}, \ldots, x_{i}\right\}$ for $i \leq k$. We may have unblocked displayed 3-separations in $M \backslash\left\{x_{1}, \ldots, x_{i}\right\}$. However by removing petals that contained a representative of $x_{j}$ for $j \in\{1, \ldots, i\}$ and not $x$, we get the required minor.

It follows from Lemma 9.2.2 that we may now add the following hypothesis.

- No proper petal of $F$ contains a representative of more than one element of $X$.

We are now almost in a position to reduce this case to the case of blocking a wheel.

Recall $J\left(P_{i}\right)$ denotes the set of joints of $P_{i}$, and $\widetilde{M}$ denotes the matroid $M$ extended by the joints of $F$.

Lemma 9.2.3. If $P_{i}$ is a petal of $F$, then for any $e \in P_{i}$, there is a minor $\widetilde{M}^{\prime}$ of $\widetilde{M}$ on $\cup\left(F-P_{i}\right) \cup e \cup J(F)$ such that the following holds:

I) if $P_{i}$ is joint-based then $\left\{J\left(P_{i}\right) \cup e\right\}$ is a circuit in $\widetilde{M}^{\prime}$ and $M^{\prime} \mid\left(\cup\left(F-P_{i}\right)\right)=$ $M \mid\left(\cup\left(F-P_{i}\right)\right)$.

II) If $P_{i}$ is rim-based then $\left\{J\left(P_{i}\right), e\right\}$ is a triangle in $\widetilde{M}^{\prime}$ and $M^{\prime} \mid\left(\cup\left(F-P_{i}\right)\right)=$ $M \mid\left(\cup\left(F-P_{i}\right)\right)$.

III) If $P_{i}$ is a 3-petal of $F$, there exist $a, b \in P_{i}$ such that

i) $\left\{a, b, J\left(P_{i}\right)\right\} \in E\left(\tilde{M}^{\prime}\right)$,

ii) one of $\{a, b\}$ parallel to the rim basepoint of $P_{i}$,

iii) one of $\{a, b\}$ is parallel to $j_{i}$ or $j_{i+1}$,

iv) $e \in\{a, b\}$. 
Proof. The case where $P_{i}$ is a 2-petal is trivial since $M \mid P_{i}$ is connected. The case where $P_{i}$ is a 3-petal is an easy corollary of Lemma 2.1 .16 .

If we reduce 3-petals in this way this may result in up to half the displayed 3separations not being blocked. However, by contracting rim elements we see that $M$ has a wheel minor with at least half as many petals as the original flower and this wheel is blocked in a star-like way.

This leads to the following lemma.

Lemma 9.2.4. There is a function $\sqrt{9.2 .4}$ such that the following holds. If $m \geq$ f9.2.4 $(t)$ then $M$ has a minor $M^{\prime}$ in which the following hold.

i) $M^{\prime} \backslash\left(E\left(M^{\prime}\right) \cap X\right)$ is a rank-t wheel,

ii) $X^{\prime}=E\left(M^{\prime}\right) \cap X$ blocks all 3-separations displayed by the canonical flower of $M^{\prime} \backslash X^{\prime}$,

iii) $X^{\prime}$ is minimal with respect to this property, and

iv) the crossing graph of $X^{\prime}$ with respect to $M^{\prime}$ is a star with at least $\frac{t}{k}$ vertices.

Proof. By Lemma 9.2.2 and Lemma 9.2.3 this holds when $f_{9.2 .4}(t)=2 t+k^{2}$

Recall that since the crossing graph of $X$ in $M$ is a star this means that there are $n^{\prime}-1$ members of $X$ that do not cross each other and one element, $x$, of $X$ that crosses all others. For the remainder of this section we add to our original hypotheses the following hypotheses:

- $M \backslash X$ is a wheel,

- Every vertical 3-separation of $M$ is blocked by some element of $X$,

- the crossing graph of $X$ in $M$ with respect to the canonical flower of $F$ is a star with at least $n^{\prime}$ elements, and

- $x \in X$ crosses all elements of $X-x$ in $M$.

Lemma 9.2.5. There is a minor of $M^{\prime}$ of $M$ such that $\left.M^{\prime} \backslash\{E(M) \cap X)\right\}$ is a wheel containing a consecutive set of joints $J_{1}$ such that:

i) no joint in $J_{1}$ is assigned colour $\gamma(x)$, 
ii) $\left|J_{1}\right|=m \geq \int 2.4 .4(n)$,

iii) if $j_{i}$ and $j_{k}$ are elements of $J_{1}$ coloured by colour $c$ and $\left[j_{i}, \ldots, j_{k}\right]_{j_{1}}$ then every element $j_{l}$ such that $\left[j_{i}, \ldots, j_{l}, \ldots, j_{k}\right]_{j_{1}}$ is assigned colour $c$,

iv) every joint in $J_{1}$ is assigned exactly one colour, and

v) $\left|\mu\left(J_{1}\right)\right| \geq \frac{m}{k}$.

Proof. This follows by Lemma 2.4.4 and noting that no colour is assigned to more than $k$ joints.

Lemma 9.2.6. Suppose $n^{\prime} \geq \sqrt{22.4 .4}(t)$. There is a minor $M^{\prime}$ of $M$ containing a consecutive set of joints $J_{1}$ such that the following hold:

i) $M^{\prime} \backslash\left(X \cap E\left(M^{\prime}\right)\right)$ is a wheel with at least $t+3$ joints,

ii) $X^{\prime}=E\left(M^{\prime}\right) \cap X$ is a minimal blocking set of $M^{\prime} \backslash X^{\prime}$,

iii) $\left|X^{\prime}\right| \geq t$,

iv) no joint in $J_{1}$ is assigned colour $\gamma(x)$,

v) $\left|J_{1}\right|=m \geq t$,

vi) if $j_{i}$ and $j_{k}$ are elements of $J_{1}$ coloured by colour $c$ then $j_{i}$ and $j_{k}$ are adjacent,

vii) every joint in $J_{1}$ is assigned exactly one colour,

viii) $\left|\mu\left(J_{1}\right)\right| \geq \frac{m}{k}$, and

ix) all colours in $\gamma(X-x)$ appear in $\mu(J)$.

Proof. It is clear that we can remove petals and blocking elements to find this minor.

Many of the following proofs are omitted. In general when this happens the lemmas are routine and often immediate corollaries of the previous lemmas and I believe it is easier to convince yourself that the lemma is true than it is to understand a proof of it. While these lemmas are essentially immediate, I believe it helps to separate them into lemmas for easy reference later on.

The following lemma follows from removing petals. The proof is elementary and is left to the reader. 
Lemma 9.2.7. There is a function 9.2 .7 such that the following holds. If $n^{\prime} \geq$ f9.2.7 $(t)$, there is a minor of $M^{\prime}$ of $M$ such that $M^{\prime} \backslash\left(X \cap E\left(M^{\prime}\right)\right)$ is a wheel with at least $t+3$ joints, $X \cap E\left(M^{\prime}\right)$ is a minimal set of blocking elements for $X$, and there is a set $J_{1}$ of at least $t$ joints of $M^{\prime}$ with a joint colouring of $J_{1}$ of one of the following forms:
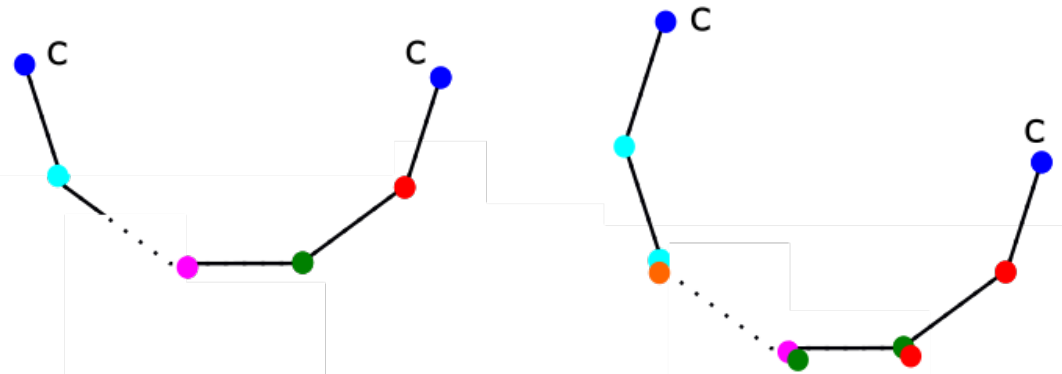

where $c=\gamma(x)$

We add to our hypotheses the following.

- the joint colouring of $F$ with respect to $X$ contains a consecutive set of joints $J_{1}$ of one of the two forms from Lemma 9.2.7 and all colours in $X$ appear on some joint of this set.

The proof of the following lemma is routine and is omitted.

Lemma 9.2.8. There is a function $\sqrt{9.2 .8}$ such that the following holds. If $n^{\prime} \geq$ $\int 9.2 .8(s, t)$, then there is either some joint in $J-J_{1}$ is coloured by at least $s$ colours, or there is a subset $X^{\prime}$ of $X$ such that in the joint colouring of $F$ in $M$ with respect to $X^{\prime}$ there is a set $J_{3}$ of $J-J_{1}$ with at least $t$ joints in which every joint is assigned exactly one colour, no joint in $J_{3}$ is assigned colour $\gamma(x)$, and if $j_{i}$ and $j_{k}$ are elements of $J_{3}$ coloured by colour $c_{i}$ then $j_{i}$ and $j_{k}$ are adjacent.

We add to our hypotheses the following. 
- In the joint colouring of $F$ with respect to $X$ a joint is either assigned every colour of $\gamma(x)$ or exactly one colour of $\gamma(X)$.

The proof of the next lemma is trivial and is omitted.

Lemma 9.2.9. If the joint coloring of $F$ in $M$ with respect to $X$ has a consecutive set $J_{2}$ in which all colours but $\gamma(x)$ appear on exactly one joint and $J_{2} \cap J_{1}=\emptyset$, then there is a minor $M^{\prime}$ of $M$ such that $X \subseteq E\left(M^{\prime}\right)$ and $M^{\prime} \backslash X$ has a swirl-like pseudo-flower, $F^{\prime} \subseteq F$, in which there is a point assigned all colours in the joint colouring of $F^{\prime}$ in $M^{\prime}$ with respect to $X$. Moreover, all colours of $(X-x)$ cross $\gamma(x)$ in the joint colouring of $F^{\prime}$ with respect to $X$.

Lemma 9.2.10. There is a function $\sqrt{9.2 .10}$ such the the following holds. If $n^{\prime} \geq$ $\int 9.2 .10(t)$ then there is a minor $M^{\prime}$ of $M$ with coindepedent set $X^{\prime}=X \cap E\left(M^{\prime}\right)$ such that $M^{\prime} \backslash X^{\prime}$ has a maximal flower $F^{\prime}$ with a joint colouring of $F^{\prime}$ of one of the following forms: 


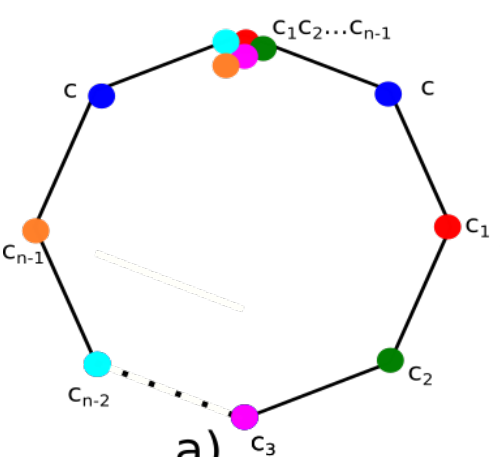

a)

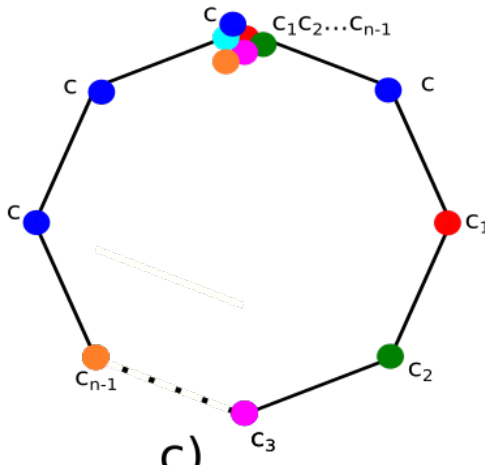

c)

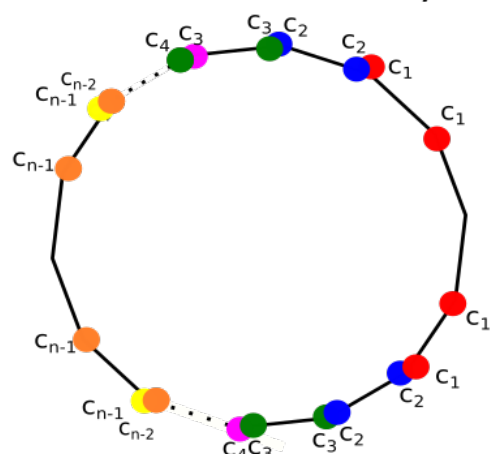

e)

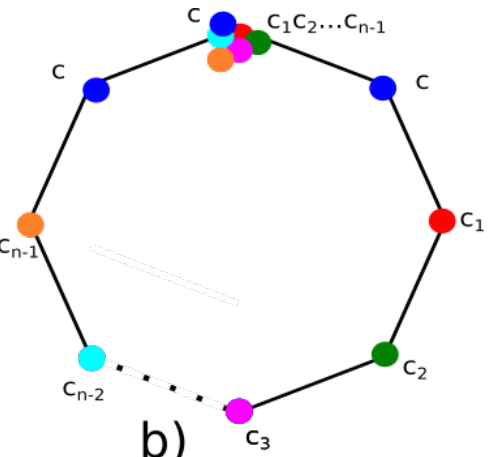

b)

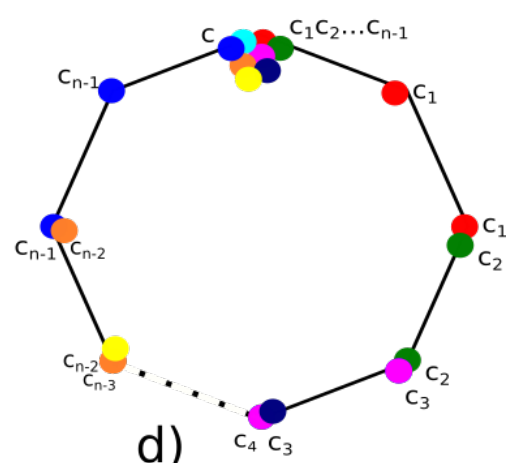

Proof. This follows from easily from Lemma 9.2.7 and Lemma 9.2.9.

We now discard the hypothesis that every joint is assigned exactly one colour or every colour and instead we add the following hypothesis.

- The joint colouring of $F$ is of one of the forms described in Lemma 9.2.10.

Lemma 9.2.11. Suppose the joint colouring of $F$ in $M$ with respect to $X$ is of the form of $a), b), c)$ from Lemma 9.2.10. Then if $n \geq t+3, M$ has a double wheel or non graphic double-wheel of rank $t$ as a minor.

Proof. Suppose the joint colouring of $F$ with respect to $X$ is of form $a$ ). The 
representation of $M$ is given below

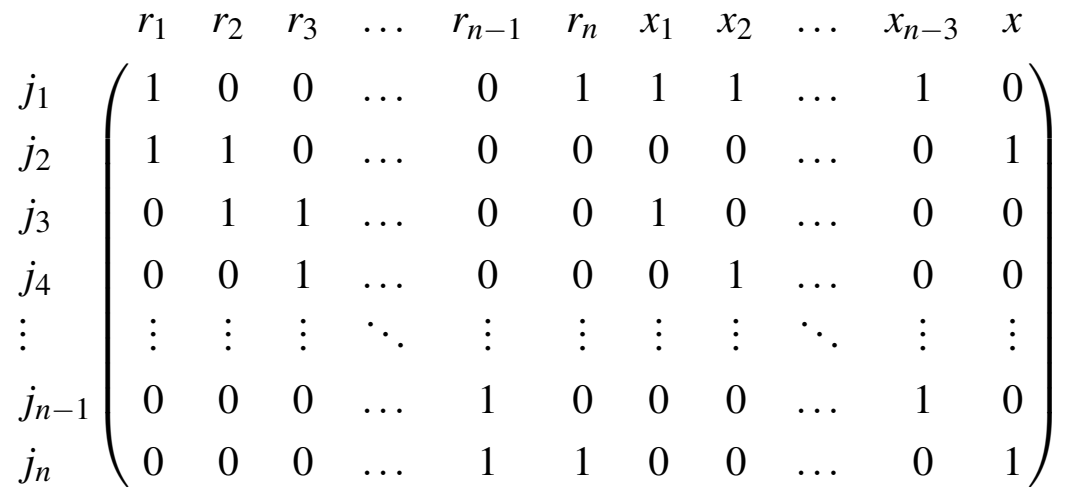

Pivot on $M_{j_{1}, r_{1}}$ and $M_{j_{n}, r_{n-1}}$ to get the following matrix:

$$
\begin{aligned}
& \begin{array}{lllllllllll}
j_{1} & r_{2} & r_{3} & \ldots & j_{n} & r_{n} & x_{1} & x_{2} & \ldots & x_{n-3} & x
\end{array} \\
& \begin{array}{l}
r_{1} \\
j_{2} \\
j_{3} \\
j_{4} \\
\vdots \\
j_{n-1} \\
r_{n-1}
\end{array}\left(\begin{array}{ccccccccccc}
1 & 0 & 0 & \ldots & 0 & 1 & 1 & 1 & \ldots & 1 & 0 \\
1 & 1 & 0 & \ldots & 0 & 1 & 1 & 1 & \ldots & 1 & 1 \\
0 & 1 & 1 & \ldots & 0 & 0 & 1 & 0 & \ldots & 0 & 0 \\
0 & 0 & 1 & \ldots & 0 & 0 & 0 & 1 & \ldots & 0 & 0 \\
0 & \vdots & \vdots & \ddots & \vdots & \vdots & \vdots & \vdots & \ddots & \vdots & \vdots \\
0 & 0 & 0 & \ldots & 1 & 1 & 0 & 0 & \ldots & 0 & 1
\end{array}\right)
\end{aligned}
$$

Deleting $j_{1}, r_{n}$ and $j_{n}$ and contracting $r_{n-1}$ gives:

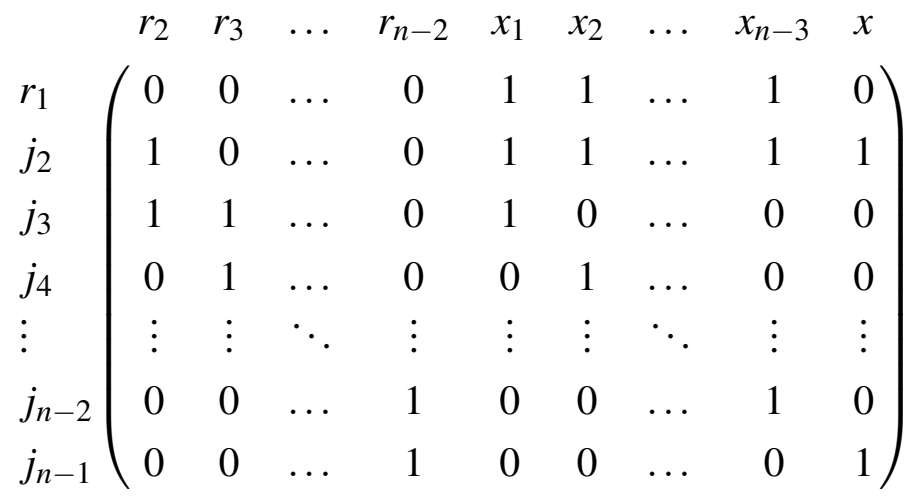

which is a representation of a double wheel as required.

Suppose the joint colouring of $F$ with respect to $X$ is of form $b$ ). Without loss of generality suppose $j_{1}$ is assigned $\gamma(x)$ and so are both $j_{n}$ and $j_{2}$. The matroid $M$ 
is represented by the matrix:

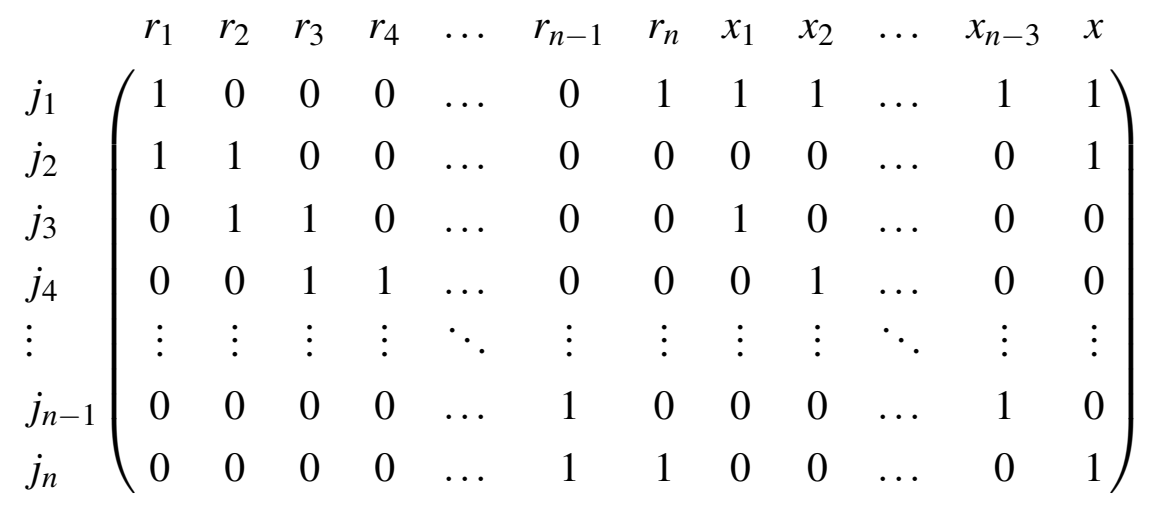

Pivoting on $M_{j_{1}, r_{1}}$ and $M j_{n}, r_{n-1}$ gives

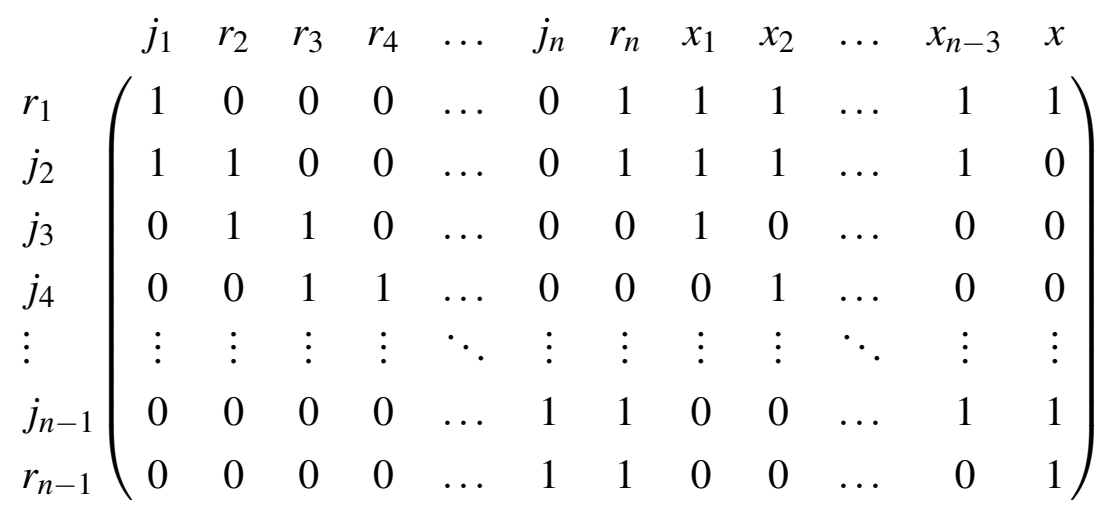

The matroid $M \backslash\left\{j_{1}, j_{n}, r_{n}\right\} / r_{n-1}$ is represented by

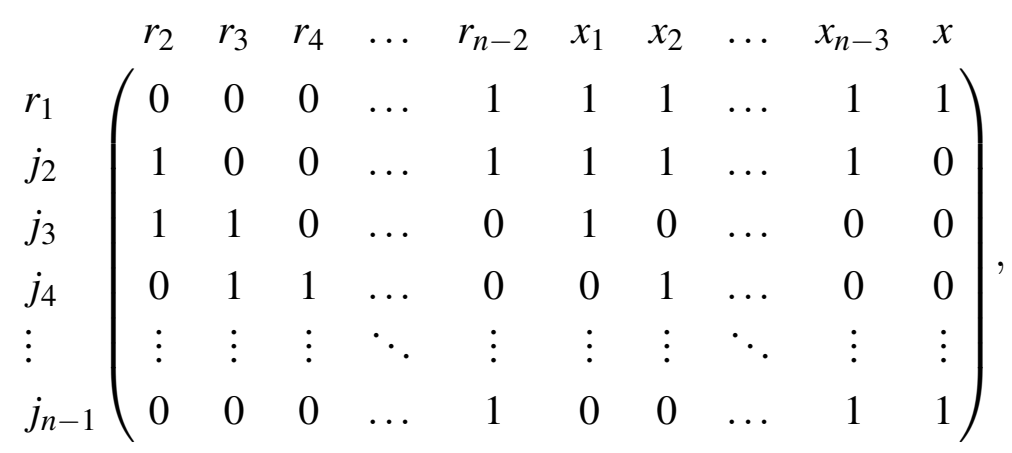

which is a representation of a non graphic double wheel.

Suppose the joint colouring of $F$ with respect to $X$ is of form $c$ ). The matroid $M$ 
is then represented by the matrix below

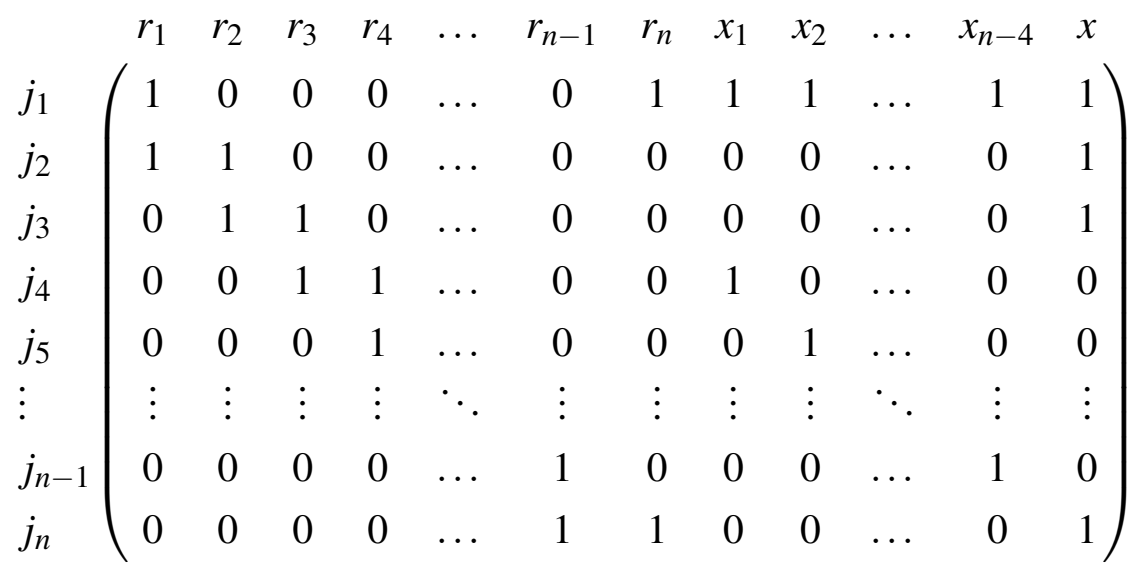

First pivot on $M_{j_{1}, r_{1}}$ to get

$$
\begin{aligned}
& \begin{array}{llllllllllll}
j_{1} & r_{2} & r_{3} & r_{4} & \ldots & r_{n-1} & r_{n} & x_{1} & x_{2} & \ldots & x_{n-4} & x
\end{array}
\end{aligned}
$$

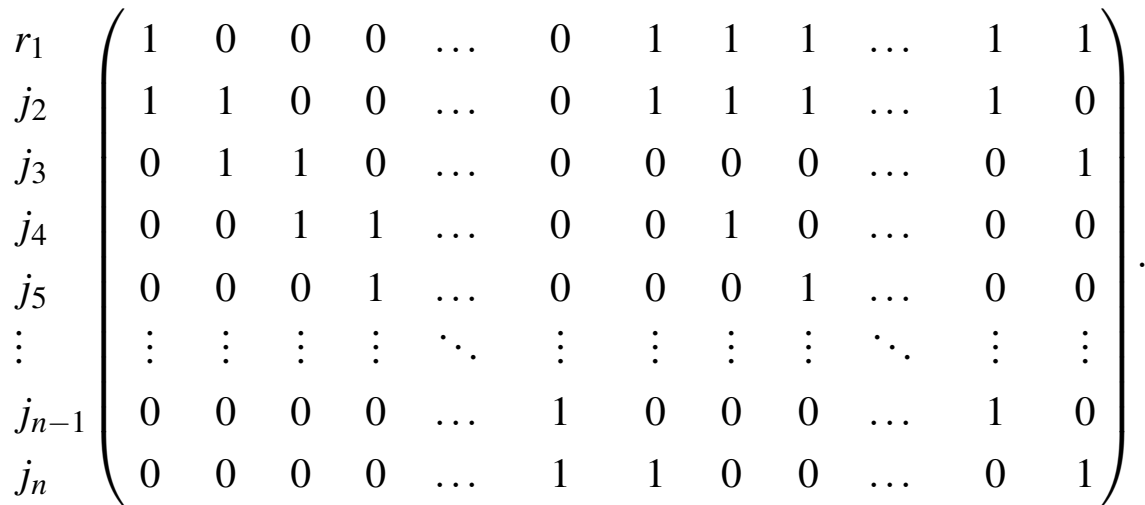

Next, pivot on $M_{j_{2}, r_{2}}$ to get

$$
\begin{aligned}
& \begin{array}{llllllllllll}
j_{1} & j_{2} & r_{3} & r_{4} & \ldots & r_{n-1} & r_{n} & x_{1} & x_{2} & \ldots & x_{n-4} & x
\end{array}
\end{aligned}
$$

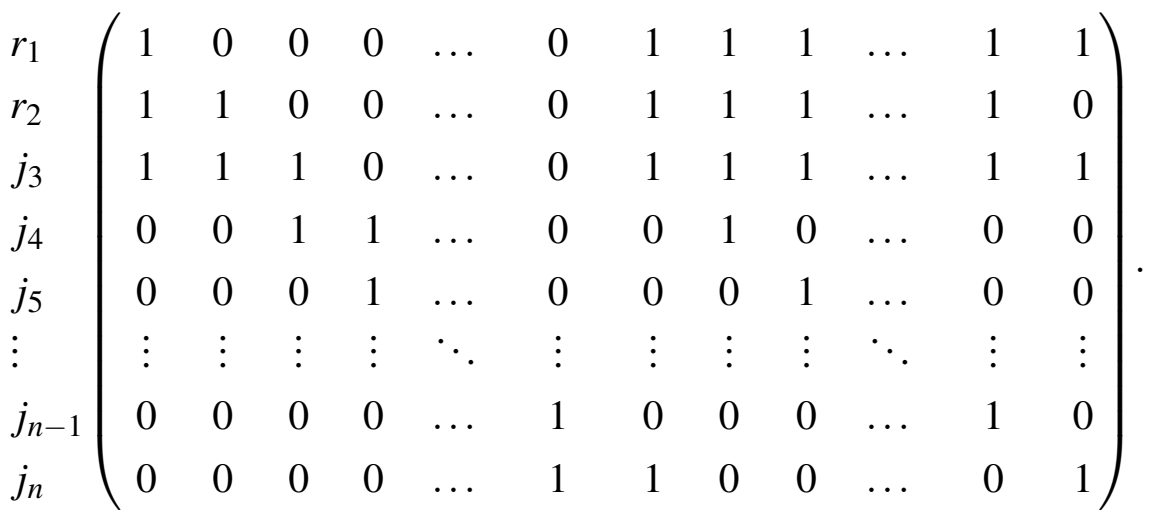


If we contract $r_{1}$ and delete $j_{1}, j_{2}$ and $r_{n}$ we get:

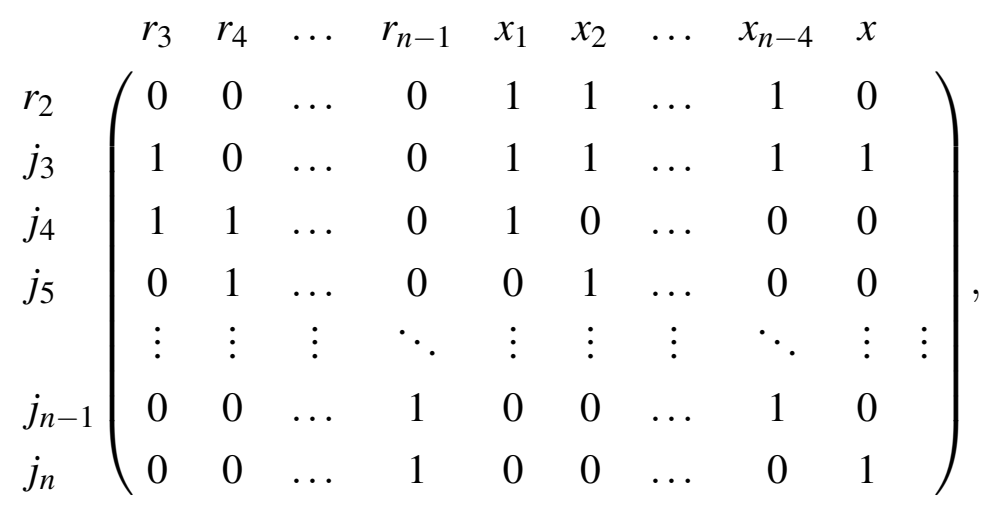

which is a representation of a double wheel.

Lemma 9.2.12. Suppose $n \geq t+4$ and the joint colouring of $F$ with respect to $X$ is of the form of d) from Lemma 9.2.10. Then M has a rank-t spike minor.

Proof. Delete $x$ and let $j_{1}$ be the joint assigned all colours of $X-x$. Take $r_{2}, \ldots, r_{n}$ and $j_{1}$ as a basis. This has the following representation:

$$
\left(\begin{array}{ccccccc}
1 & 1 & 1 & \ldots & 1 & 1 & 0 \\
1 & 0 & 0 & \ldots & 0 & 1 & 1 \\
0 & 1 & 0 & \ldots & 0 & 1 & 1 \\
0 & 0 & 1 & \ldots & 0 & 1 & 1 \\
\vdots & \vdots & \vdots & \ddots & \vdots & \vdots & \\
0 & 0 & 0 & \ldots & 1 & 1 & 1
\end{array}\right)
$$

which is a reduced standard representation of a spike.

The following well-known fact about fans has an easy, and omitted, proof.

Lemma 9.2.13. If $\left(f_{1}, \ldots f_{n}\right)$ is a fan where $\left\{f_{1}, f_{2}, f_{3}\right\}$ and $\left\{f_{n}, f_{n-1}, f_{n-2}\right\}$ are triangles, then $\left\{f_{1}, f_{2}, f_{4}, f_{6}, \ldots, f_{n-1}, f_{n}\right\}$ is an independent set.

Notice that if we contract two non-adjacent rim elements of a wheel $M$ the groundset of $E(M)-\left\{j_{1}, j_{2}\right\}$ is partitioned into two disjoint fans in $M /\left\{j_{1}, j_{2}\right\}$. 
Lemma 9.2.14. Suppose $F$ has an even number of joints, and suppose there is some partition of $E(M)-X$ into two sets $\left\{j_{1}, r_{1}, j_{2}, r_{2}, \ldots, j_{n-1}, r_{n-1}, j_{n}\right\}$ and $\left\{r_{n}, j_{n+1}, r_{n+1}, \ldots, j_{2 n}, r_{2 n}\right\}$ such that the following holds: the element $x_{i}$ is in a triangle with $r_{j}$ and $r_{k}$ for some $j \in\{1, \ldots, n-1\}$ and some $k \in\{n+1, \ldots, 2 n-1\}$, and, if $x_{a} \neq x_{b}$, then the triangles containing $x_{a}$ and $x_{b}$ are distinct. Then $M$ has an $M^{*}\left(K_{3, n-1}\right)$-minor.

Proof. Contract $j_{1}, j_{n}, j_{i+1}, j_{2 n}$. Then $j_{2}, r_{2}, r_{n}, \ldots r_{n-1}, r_{n-2}, j_{n-1}$ is a cycle and $j_{n+1}, r_{n+2}, r_{n+3}, \ldots, r_{2 n-3}, r_{2 n-2}, j_{2 n-1}$ is a cycle, and $X$ is a matching between two disjoint cycles. Therefore by Lemma 4.1.2 $M$ has an $M^{*}\left(K_{3, n-1}\right)$-minor.

Lemma 9.2.15. Suppose the joint colouring of $F$ with respect to $X$ is of form $e$ ) of Lemma 9.2.10 Then $M$ has an $M^{*}\left(K_{3, \frac{n}{2}}\right)$-minor.

Proof. This follows from Lemma 9.2.14.

The proof of Theorem 9.2.1 is now routine.

Theorem 9.2.1. There is a function 9.2 .1 such that for all $t \geq 5$ the following holds. If $M$ is a binary matroid with a coindependent set $X$ such that

I) $M \backslash X$ has a maximal swirl-like pseudo-flower of order $n$ where $n \geq \sqrt{9.0 .1}(t)$,

II) every 3-separation of $M$ displayed by $F$ is blocked by an element of $X$,

III) the crossing graph of $X$ with respect to $F$ in $M$ is a star,

$I V)$ there is no $x \in X$ that contains a representative in $k$ or more petals,

then $M$ has minor isomorphic to one of the following:

i) a rank-t spike,

ii) a rank-t double wheel,

iii) a rank-t non graphic double wheel,

iv) $M^{*}\left(K_{3, t}\right)$. 
Proof. Let $f_{9.2 .1}(t)=\int_{9.2 .2}\left(\int_{9.2 .4}(m)\right)$. By Lemma 9.2.2 there is a minor $M_{1}$ of $M$ such that the following hold.

$M_{1} \backslash\left(E\left(M_{1}\right) \cap X\right)$ has a swirl-like pseudo-flower $F_{1} \subseteq F$,

$E\left(M_{1}\right) \cap X=X_{1}$ is a coindependent set that is a minimal blocking set of $F_{1}$,

$F_{1}$ has order at least $\int 9.2 .4(m)$,

$F_{1}$ is such that no proper petal contains a representative of more than one element of $X_{1}$, and

the crossing graph of $X_{1}$ in $F_{1}$ with respect to $M_{1}$ is a star. By Lemma 9.2.4 $M_{1}$ has a minor $M_{2}$ in which the following hold.

i) $M_{2} \backslash\left(E\left(M_{2}\right) \cap X\right)$ is a rank- $m$ wheel,

ii) $X_{2}=E\left(M_{2}\right) \cap X$ blocks all 3-separations displayed by the canonical flower of $M_{2} \backslash X_{2}$,

iii) $X_{2}$ is minimal with respect to this property, and

iv) the crossing graph of $X_{2}$ with respect to $M_{2}$ is a star with at least $\frac{m}{k}$ vertices.

Since $m=\int 9.2 .7\left(f_{? ?}\left(s_{1}, s_{2}\right)\right)$ it follows from Lemma 9.2.7 that there is a minor of $M_{3}$ of $M_{2}$ such that $M_{3} \backslash\left(X \cap E\left(M_{3}\right)\right)$ is a wheel with at least $\int \frac{0.2 .8}{6}\left(s_{1}, s_{2}\right)+3$ joints, $X \cap E\left(M^{\prime}\right)$ is a minimal set of blocking elements for $X$, and there is a set

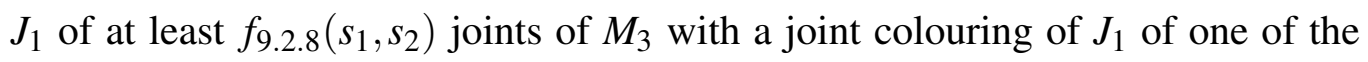
following forms:
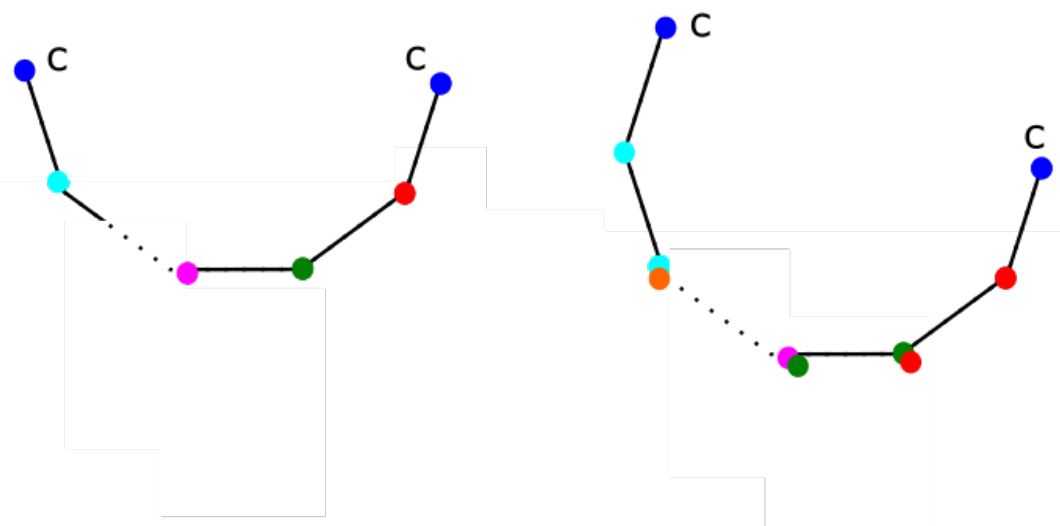
where $c=\gamma(x)$ when $x$ is the element crossing all others. By Lemma 9.2.8 there is either some joint in $J-J_{1}$ is coloured by at least $s_{1}$ colours, or there is a subset $X_{4}$ of $X_{3}$ such that in the joint colouring of $F_{3}$ in $M_{3}$ with respect to $X_{4}$ there is a set $J_{3}$ of $J-J_{1}$ with at least $s_{2}$ joints in which every joint is assigned exactly one colour, no joint in $J_{3}$ is assigned colour $\gamma(x)$, and if $j_{i}$ and $j_{k}$ are elements of $J_{3}$ coloured by colour $c_{i}$ then $j_{i}$ and $j_{k}$ are adjacent.

Since $s_{1}, s_{2} \geq \sqrt{9.2 .10}(2 t)$ it follows from Lemma 9.2 .10 there is a minor $M_{5}$ of $M_{3}$ with coindepedent set $X_{5}=X \cap E\left(M_{5}\right)$ such that $M_{5} \backslash X_{5}$ has a maximal flower $F_{5}$ with a joint colouring of $F_{5}$ of one of the following forms:

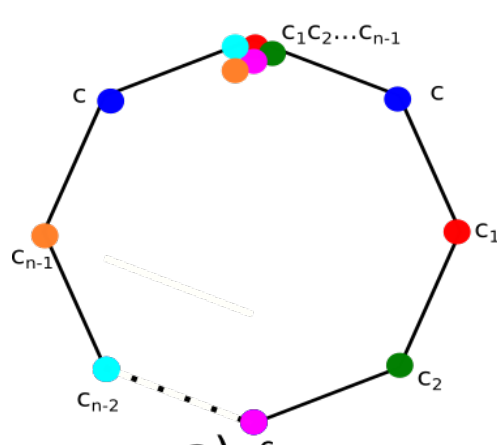

a)

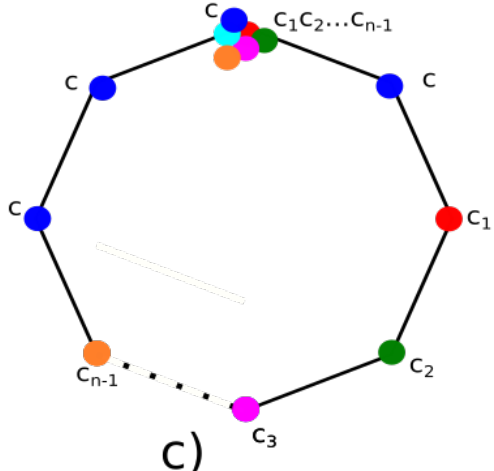

C)

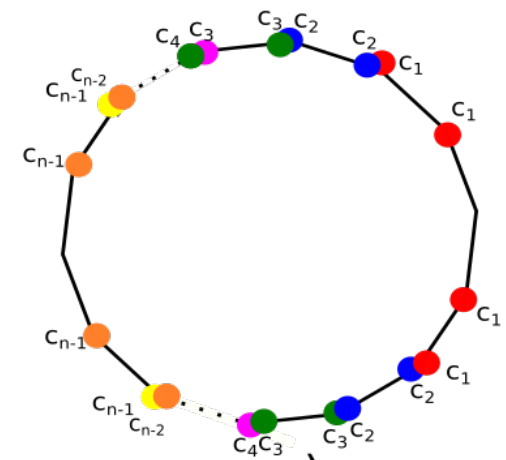

e)
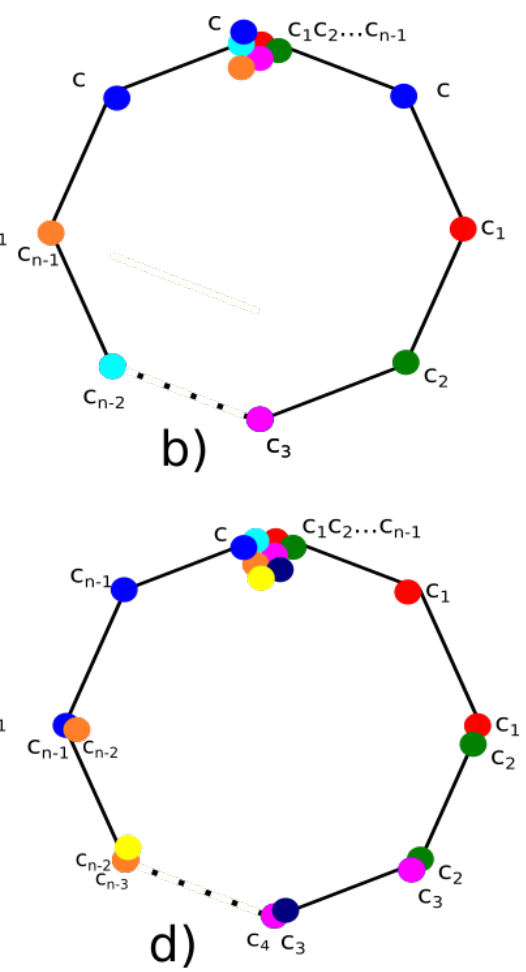

d)

(1)




\subsection{Complete Graphs}

In this section we prove the following theorem.

Theorem 9.3.1. There is a function $\sqrt{9.3 .1}$ such that for all $t \geq 5$ the following holds. If $M$ is a binary matroid with a coindependent set $X$ such that

I) $M \backslash X$ has a maximal swirl-like pseudo-flower of order $n$ where $n \geq \sqrt{9.3 .1}(t)$,

II) every 3-separation of $M$ displayed by $F$ is blocked by an element of $X$,

III) the crossing graph of $X$ with respect to $F$ in $M$ is a complete graph,

$I V)$ there is no $x \in X$ that contains a representative in $k$ or more petals for some $k \in \mathbb{Z}_{>0}$.

then $M$ has a minor isomorphic to one of the following:

i) a rank-t spike,

ii) a rank-t double wheel,

iii) a rank-t non graphic double wheel,

iv) $M^{*}\left(K_{3, t}\right)$.

In this section we work under the hypotheses of Theorem 9.3.1, that is we add the following hypothesis to our previous hypotheses.

- The crossing graph of $X$ with respect to $F$ in $M \backslash X$ is a complete graph, and

- no element $x \in X$ contains a representative in $k$ or more petals.

We can also without loss of generality assume that all crossing elements are distinguishable from the joint colouring of $F$ in $M$ with respect to $X$. Therefore we add the following hypothesis.

- Every element of $X$ is distinguishable from every other element of $X$ by the joint colouring of $F$ in $M$ with respect to $X$.

Recall that the elements of $X$ can either cross in the colouring of the basepoints of $F$ or they can cross by having representatives in the same petal. 
Definition 9.3.2. Let $F$ be a swirl-like pseudo-flower and $X$ a set of blocking elements of $F$. We say that an element $x \in X$ is strongly represented in a petal $P$ of $F$ if $F(x)$ contains an element of $P$ that is not parallel to a joint of $P$, or $F(x)$ contains elements parallel to two joints of $P$.

For some $m \in \mathbb{Z}_{\geq 0}$ we say that $F$ contains a $m$-big petal if $F$ has a minimal petal in which at least $m$ elements of $X$ are strongly represented.

We can view a colouring of a swirl-like pseudo-flower as a hypergraph, with the joints the vertices and the colours the edges. That is, if there are $k$ joints are coloured by some $c$ then let the set of these joints be $S$. The set $S$ is then an edge in the hypergraph. This means that we can now use the language of matchings.

The next lemma involves an infinite family of functions. This could be rewritten (as my supervisor would prefer) in terms of a single function with an extra variable.

Lemma 9.3.3. For $i \in \mathbb{Z}_{\geq 1}$ let $\sqrt{9.3 .3} i$ be the function such that $\sqrt{9.3 .3, i}(t, l)=$ $\int_{2.4 .4}(t, \sqrt{9.3 .3}[-1(t, l))$ and $\sqrt{9.3 .3}, 1=\sqrt{2.4 .4}$. Suppose the joint colouring of $F$ with respect to $X$ uses at least $\int 9.3 .3 k(t, l)$ colours and no colour is assigned to more than $k$ points. Then there is a minor, $M^{\prime}$, of $M$ with a swirl-like pseudo-flower $F^{\prime} \subseteq F$ that is blocked by a set $X^{\prime}=X \cap E\left(M^{\prime}\right)$ with the following properties:

i) $\left|X^{\prime}\right| \geq \min \{t, l\}$

ii) the joint colouring of $F^{\prime}$ with respect to $X^{\prime}$ is such that every joint is assigned either all colours in $\gamma\left(X^{\prime}\right)$ or exactly one colour of $\gamma\left(X^{\prime}\right)$, and

iii) the crossing graph of the elements of $X^{\prime}$ is a complete graph.

Proof. If $k-1$ points are assigned $n$ colours in common then, since all colours are distinguishable from the joint colouring, the remaining colours must be contained on distinct points and the result follows. Assume some point is assigned at least f9.3.3 $i(t, l)$ colours. By Lemma 2.4.4 either there is a matching using $t$ colours or a joint assigned at least $\sqrt{9.3 .3}, i-1(t, l)$ colours. If there is a matching using $t$ colours then there is a minor $M^{\prime}$ of $M$ with a coindependent set $X^{\prime} \subseteq X \cap E\left(M^{\prime}\right)$ such that the following hold:

i) $M^{\prime} \backslash X^{\prime}$ has a swirl-like pseudo-flower $F^{\prime}$ in which every displayed 3separation is blocked by an element of $X^{\prime}$, 
ii) the crossing graph of $X^{\prime}$ with respect to $F^{\prime}$ in $M^{\prime}$ is a complete graph, and

iii) every joint in $F^{\prime}$ is assigned either all colours or exactly one colour.

As at most $k-1$ points are coloured by the same colour and all blocking elements are distinguishable from the joint colouring we must at some point see such a matching. The result then follows.

The following lemma is a routine corollary of Lemma 9.3.3 and is left to the reader.

Lemma 9.3.4. There is a function $\sqrt{9.3 .4}$ such that the following holds. If $n \geq$ 9.3.4 $(t)$ then $M$ has a minor $M^{\prime}$ such that if $X^{\prime}=X \cap E\left(M^{\prime}\right)$ the following hold:

i) $M^{\prime} \backslash X^{\prime}$ has a swirl-like pseudo-flower $F^{\prime} \subseteq F$ of order $t$,

ii) any displayed 3-separation of $F^{\prime}$ in $M^{\prime}$ is blocked by some $x \in X^{\prime}$,

iii) the crossing graph of $X^{\prime}$ with respect to $F^{\prime}$ in $M^{\prime}$ is a complete graph,

iv) the joint colouring of $F^{\prime}$ in $M^{\prime}$ with respect to $X^{\prime}$ is such that every joint is coloured by either all colours in $\gamma\left(X^{\prime}\right)$ or exactly one colour in $\gamma\left(X^{\prime}\right)$, and

v) every proper petal in $F^{\prime}$ is either $\left|X^{\prime}\right|$-big or contains a representative of exactly one element of $X^{\prime}$.

For the remainder of this section we work under the following additional hypotheses.

- the joint colouring of $F$ in $M$ with respect to $X$ is such that every joint is coloured by either all colours in $\gamma(X)$ or exactly one colour in $\gamma(X)$, and

- every proper petal in $F$ is either $\left|X^{\prime}\right|$-big or contains a representative of exactly one element of $X$.

We split into two cases, one where every petal contains a representative of exactly one element of $X$ and one where $F$ contains an $n^{\prime}$-big petal. 


\subsubsection{No Big Petal}

Throughout this subsection we work under the following additional hypothesis

- Every petal of $F$ contains a representative of exactly one element of $X$.

Lemma 9.3.5. There is a function 90.3 .5 such that the following holds. If $n \geq$ $9.3 .5(t)$, then $M$ has a minor $M^{\prime}$ with a coindependent set $X^{\prime}$ such that $M^{\prime} \backslash X^{\prime}$ is a wheel, every vertical 3-separation of $M^{\prime} \backslash X^{\prime}$ is blocked by an element of $X^{\prime}$, and the crossing graph of $X^{\prime}$ with respect to $M^{\prime} \backslash X^{\prime}$ is a complete graph.

Proof. The case where $P_{i}$ is a 2-petal is trivial since $M \mid P_{i}$ is connected. The case where $P_{i}$ is a 3-petal is an easy corollary of Lemma 2.1.16.

Throughout the remainder of this subsection we work under the following additional hypothesis

- $M \backslash X$ is a wheel.

- $F$ is the canonical flower of $M \backslash X$.

Lemma 9.3.6. There is a function $\sqrt{9.3 .6}$ such that if $n \geq \sqrt{9.3 .6}(t)$ the following holds. There is a minor $M^{\prime}$ of $M$ such that $M^{\prime} \backslash\left(X \cap E\left(M^{\prime}\right)\right)$ is a rank-m wheel, $\left|X \cap E\left(M^{\prime}\right)\right|=t$, and the joint colouring of the canonical flower of $M^{\prime} \backslash(X \cap$ $\left.E\left(M^{\prime}\right)\right)$ has a set $J_{1}$ of one of the following forms: 


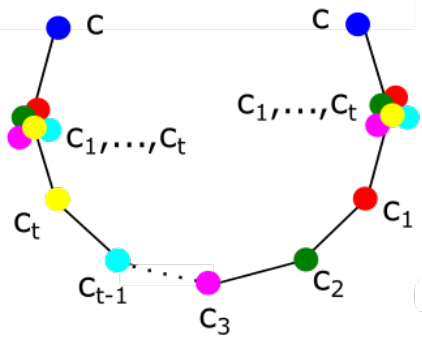

i)

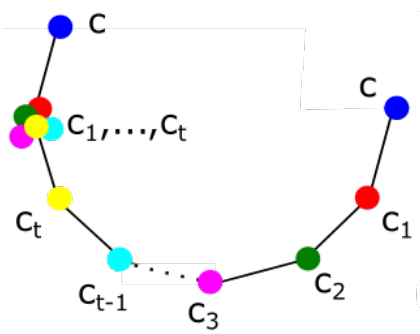

iii)

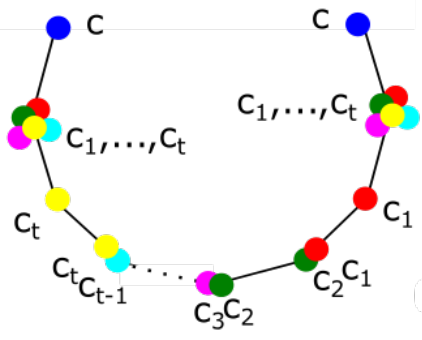

v)

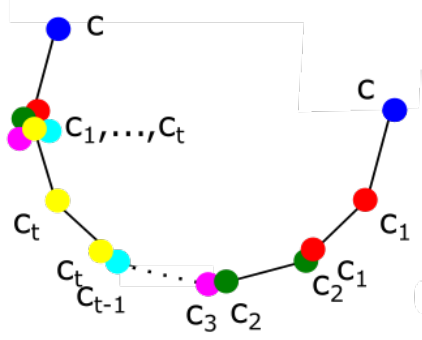

vii)

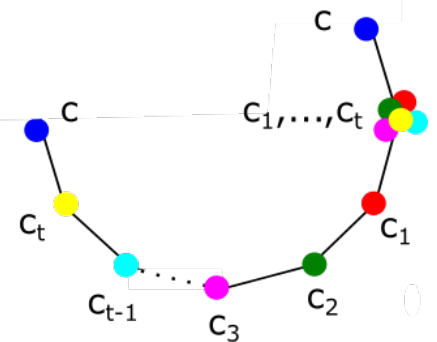

ii)

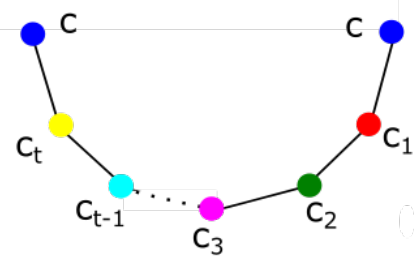

iv)

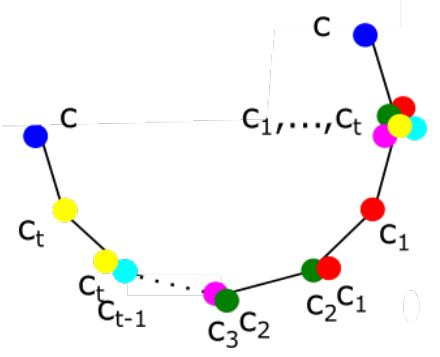

vi)

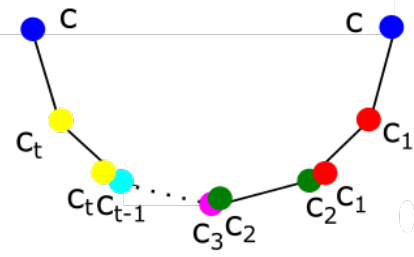

viii)

and every element of $\left(X \cap E\left(M^{\prime}\right)\right)-\left\{\gamma^{-1}(c)\right\}$ crosses $\gamma^{-1}(c)$.

Proof. Since every element of $X$ is distinguishable from the joint colouring of $F$, this follows from Lemma 2.4.7. 
We say that a joint that is coloured by all colours is a rainbow joint.

Lemma 9.3.7. There is a function $\sqrt{9.3 .7}$ such that if $n \geq \sqrt{9.3 .7}(t, l)$ then the following holds.

i) There is a minor $M^{\prime}$, of $M$ with a swirl-like pseudo-flower $F^{\prime} \subseteq F$ of order $t$ blocked by a set $X^{\prime}=X \cap E\left(M^{\prime}\right)$ such that $F^{\prime}$ has at least 2 rainbow joints in the joint colouring of $F^{\prime}$ in $M^{\prime}$ with respect to $X^{\prime}$ and a set of the following form:
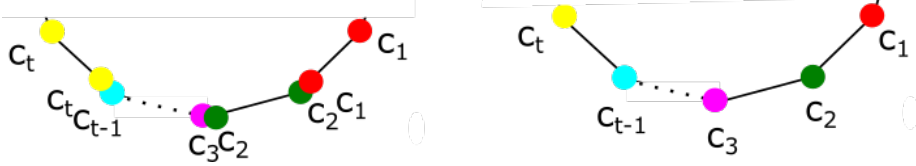

or

ii) There is a minor $M^{\prime}$, of $M$ with a swirl-like pseudo-flower $F^{\prime} \subseteq F$ of order $l$ blocked by a set $X^{\prime}=X \cap E\left(M^{\prime}\right)$ such that the joint colouring of $F^{\prime}$ with respect to $X^{\prime}$ is of one of the following forms: 

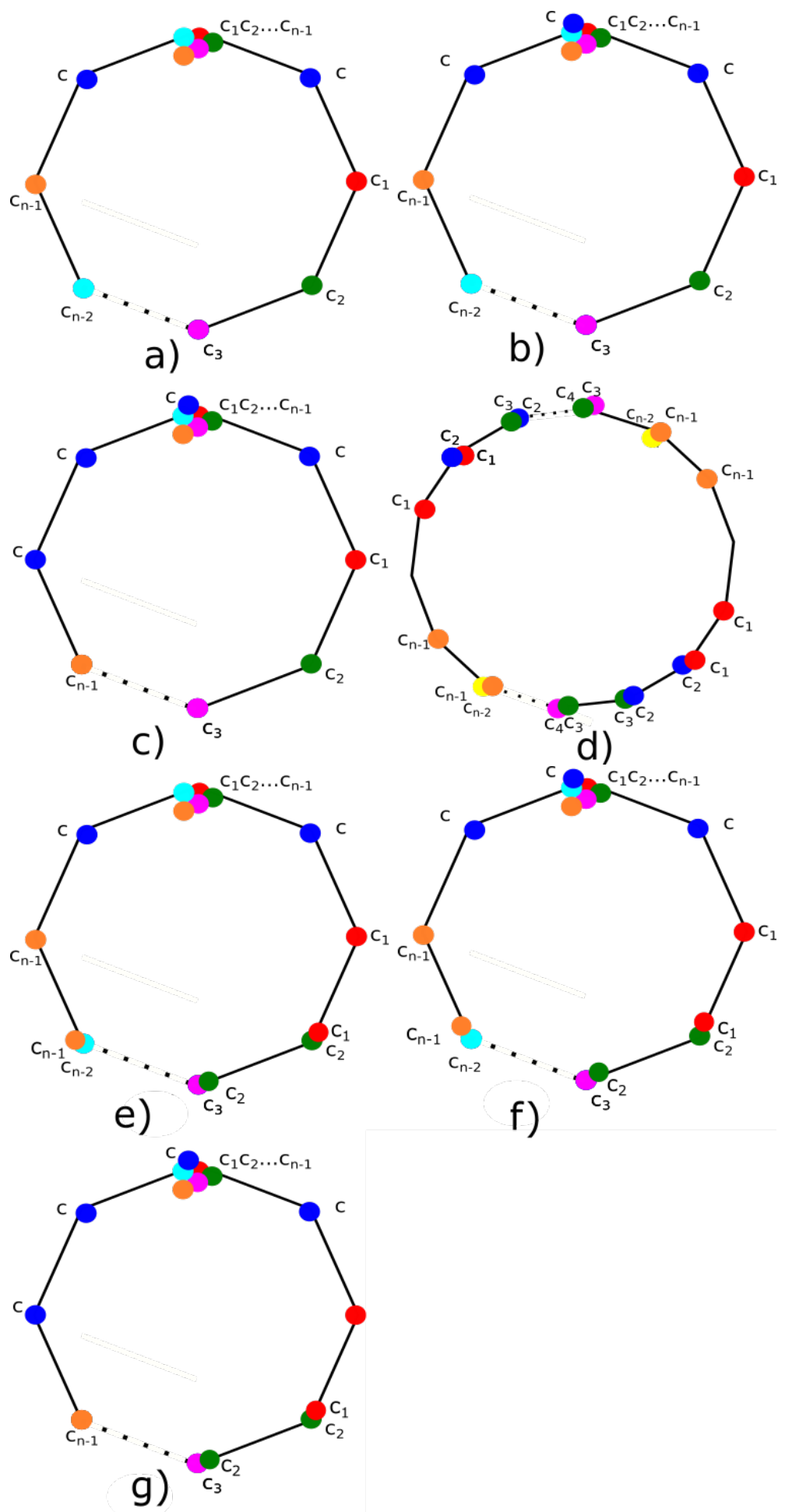

Proof. Suppose $n \geq \sqrt{9.3 .6}(m)$ where $\left.m \geq f_{\text {ref } z x q}(t)\right\}$, and $\gamma^{-1}(c)=x$. By Lemma 9.3.6 there is a minor $M_{1}$ of $M$ such that $M_{1} \backslash\left(X \cap E\left(M_{1}\right)\right)$ has a swirl-like pseudo-flower $F_{1}$, and the joint colouring of $F_{1}$ with respect to $X_{1}$ contains a con- 
secutive set $J_{1}$ of joints using $m$ colours of one of forms i)-viii) from Lemma 9.3.6 where $\gamma\left(X_{1}\right)=\left\{c_{1}, \ldots, c_{m}, c\right\}$. If the joint colouring is of form i) or v) then the lemma follows. Assume that the joint colouring is of form ii) iii) or iv) vi) vii) viii).

Let $J^{\prime}=J\left(F_{1}\right)-J_{1}$. Since every element of $X-x$ crosses $x$, one of the following holds:

1. $J^{\prime}$ contains exactly one rainbow joint,

2. $J^{\prime}$ contains more than one rainbow joint, or

3. there is a minor $M_{2}$ of $M$ such that $M_{2} \backslash\left(X \cap E\left(M_{2}\right)\right)$ has a swirl-like pseudoflower $F_{2}$ of order $m^{\prime}$, and the following holds:

- the joint colouring of $F_{2}$ with respect to $X_{2}$ contains a consecutive set $J_{2}$ of joints using $t$ colours $\left\{c_{1}, \ldots, c_{t}\right\}$ such that $J_{3}$ is of one of the forms of Lemma 9.3.6, and $J_{1} \cap J_{2}=\emptyset$.

If $J^{\prime}$ contains a rainbow joint then the result follows.

It follows from a routine case analysis that we may remove petals with basepoints in $J_{1}$ or $J_{2}$ to obtain a minor $M^{\prime}$ of $M_{2}$ such that $M^{\prime} \backslash\left(X \cap E\left(M^{\prime}\right)\right)$ has a swirl-like pseudo-flower $F^{\prime}$ with at least two rainbow joints and a set of the following form:
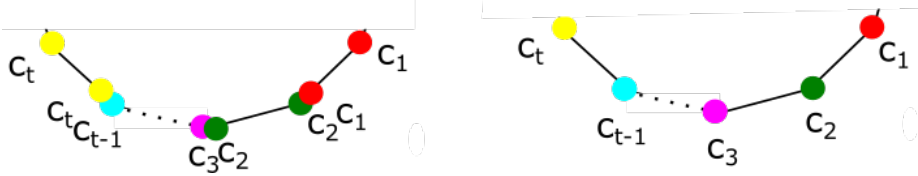

or the joint colouring of $F^{\prime}$ with respect to $X \cap E\left(M^{\prime}\right)$ is of one of the forms a),b),c),d),e),f) above.

Lemma 9.3.8. There is a function $\sqrt{9.3 .8}$ such that the following holds. If $n \geq$ $\int 9.3 .8(t)$ and the joint colouring of $F$ with respect to $X$ is as in $(d)$ of Lemma 9.3.7 then $M$ has an $M^{*}\left(K_{3, t}\right)$-minor.

Proof. This follows from Lemma 9.2.14 
Lemma 9.3.9. There is a function 9.3 .9 such that the following holds. If $n \geq$ f9.3.9 $(t)$ and the joint colouring of $F$ with respect to $X$ is as in $(a),(b),(c),(e)$, $(f)$, or $(g)$ of Lemma 9.3.7, then $M$ has a minor isomorphic to a rank-t spike, a rank-t double wheel, a rank-t non graphic double wheel or $M^{*}\left(K_{3, t}\right)$.

Proof. This follows from Theorem 9.2.1.

Lemma 9.3.10. There is a function $\sqrt{9.3 .10}$ such that if $n \geq \sqrt{9.3 .10}(t)$, the following holds. Suppose that the joint colouring of $F$ in $M$ with respect to $X$ has at least two rainbow joints. Then $M$ has a minor $M^{\prime}$ such that $M^{\prime} \backslash\left(E\left(M^{\prime}\right) \cap X\right)$ has a swirl-like pseudo-flower $F^{\prime}, F^{\prime}$ is blocked by $E\left(M^{\prime}\right) \cap X$ and $F^{\prime}$ has a $t^{\prime}$-big petal $P$ where $t^{\prime} \geq \frac{t}{k}$ and all colours of $\gamma\left(X^{\prime}\right)$ appear on at least one joint that is not a joint of $P$.

Proof. Let $j_{1}$ and $j_{i}$ be rainbow joints. If $j_{1}$ and $j_{i}$ are adjacent then the result follows easily by noting that the rim-based 2 petal with basepoint $r \in \operatorname{cl}\left\{j_{1}, j_{i}\right\}$ is an $n^{\prime}$-big petal. Suppose $j_{1}$ and $j_{i}$ are not adjacent then either $\left[j_{1}, \ldots, j_{i}\right]_{j_{1}}$ or $\left[j_{i}, \ldots, j_{1}\right]_{j_{1}}$ is a set of joints containing at least $\frac{|X|}{2}$ colours, and at least half of the colours contained in this set aredistinguishable by this set. Without loss of generality suppose $\left[j_{1}, \ldots, j_{i}\right]_{j_{1}}$ is such a section. Remove all petals in $\left[j_{i}, \ldots, j_{1}\right]_{j_{1}}$ that do not have $j_{1}$ or $j_{i}$ as a joint. There is now exactly one joint $j_{n}$ such that $\left[j_{i}, j_{n}, j_{1}\right]_{j_{1}}$. If this joint is not a rainbow joint then remove any petal with $j_{n}$ as a joint. The resulting flower then has a joint colouring with either two or three consecutive rainbow joints. Both of these cases give rise to a minor $M^{\prime}$ of $M$ with swirl-like pseudo-flower $F^{\prime}$ that has an $\frac{|X|}{8}$-big petal $P$ and every colour appears on at least one joint that is not a joint of $P$.

The proof of the next theorem is now routine and left to the reader.

Lemma 9.3.11. There is a function 9.9 .11 such that the following holds. If $n \geq$ f9.3.11 $(t)$ then either

- M has a minor $M^{\prime}$ with coindepedent set $X^{\prime}$ such that the following hold:

i) $M^{\prime} \backslash X^{\prime}$ is a wheel,

ii) $X^{\prime}$ blocks all vertical 3-separations of $M^{\prime} \backslash X^{\prime}$,

iii) $\left|X^{\prime}\right| \geq t$ 
iv) the joint colouring of $M^{\prime} \backslash X^{\prime}$ has at least two rainbow joints and all colours distinguishable from the joint colouring,

or

- M has a minor isomorphic to one of the following.

i) a rank-t double wheel,

ii) a rank-t non graphic double wheel,

iii) $M^{*}\left(K_{3, t}\right)$,

iv) a rank-t spike.

\subsubsection{Big Petal}

In this subsection we work under the following additional hypothesis,

- $F$ has an $n$-big petal.

There are at most $k n$-big petals in $F$ so there must be some set $J_{1}$ of $F$ in which all joints in $J_{1}$ are assigned exactly one colour and $J_{1}$ contains at least $\frac{n}{k}$ colours. Remove all colours not appearing in this set. Let the set $J_{1}$ be $\left[j_{1}, \ldots, j_{i}\right]_{j_{1}}$. Let $j_{a}$ and $j_{b}$ be two rainbow joints with minimal distance between them. Contract all rim elements between joints in $\left[j_{i}, \ldots, j_{n}\right]_{j_{1}}$ that are not adjacent to $j_{a}$ and $j_{b}$. Am (omitted) case analysis shows that we can either reduce this to a case where we have one big petal or where we have a wheel with three joints in the closure of all fundamental circuits of the blocking elements. Combining this with Lemma 2.4.4 we get the following two lemmas.

Lemma 9.3.12. There is a function $\sqrt{9.3 .12}$ such that the following holds. Suppose that $n \geq \sqrt{9.3 .12}(t)$ and $F$ has an n-big joint-based 2-petal. There is a minor $M^{\prime}$ of $M$ such that $M^{\prime} \backslash\left(X \cap E\left(M^{\prime}\right)\right)$ has a swirl-like pseudo-flower $F^{\prime}$ of order $t$, every 3 separation of $M^{\prime} \backslash\left(X \cap E\left(M^{\prime}\right)\right)$ is blocked by an element of $X^{\prime}$ and the joint colouring of $F^{\prime}$ with respect to $X^{\prime}$ is of one of the following forms: 

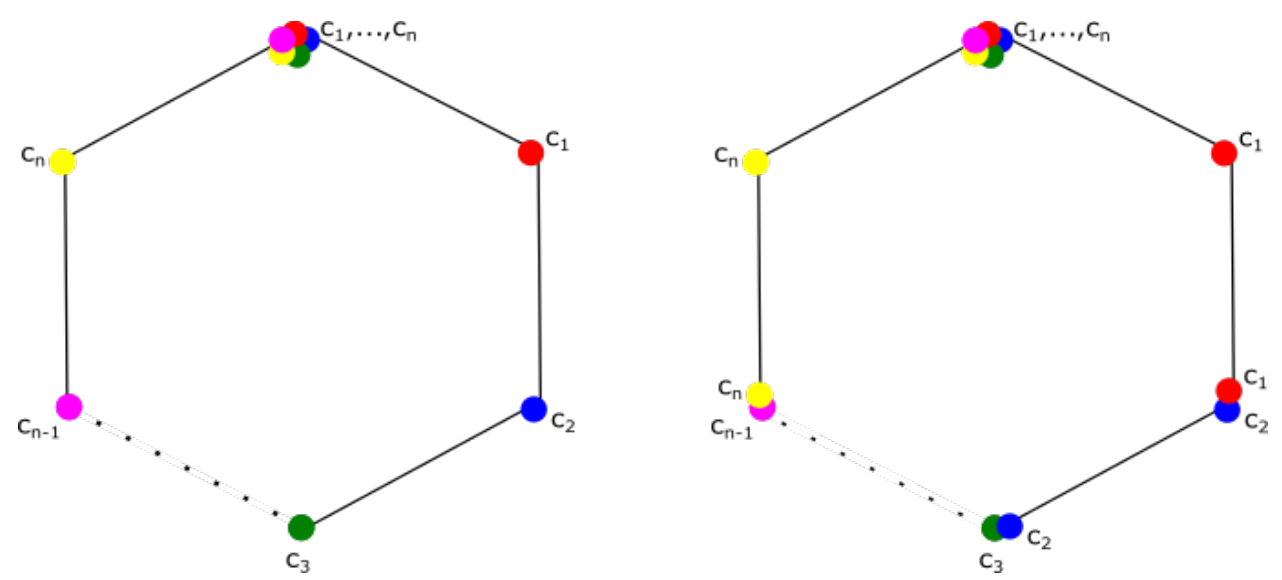

Lemma 9.3.13. There is a function 9.3 .13 such that the following holds. Suppose that $n \geq \sqrt{9.3 .13}(t)$ and $F$ has an n-big rim-based 2-petal or 3-petal. There is a minor $M^{\prime}$ of $M$ with such that $M^{\prime} \backslash\left(X \cap E\left(M^{\prime}\right)\right)$ has a swirl-like pseudo-flower $F^{\prime}$ of order $t$, every 3 separation of $M^{\prime} \backslash\left(X \cap E\left(M^{\prime}\right)\right)$ is blocked by an element of $X^{\prime}$ and the joint colouring of $F^{\prime}$ with respect to $x^{\prime}$ is of one of the following forms:
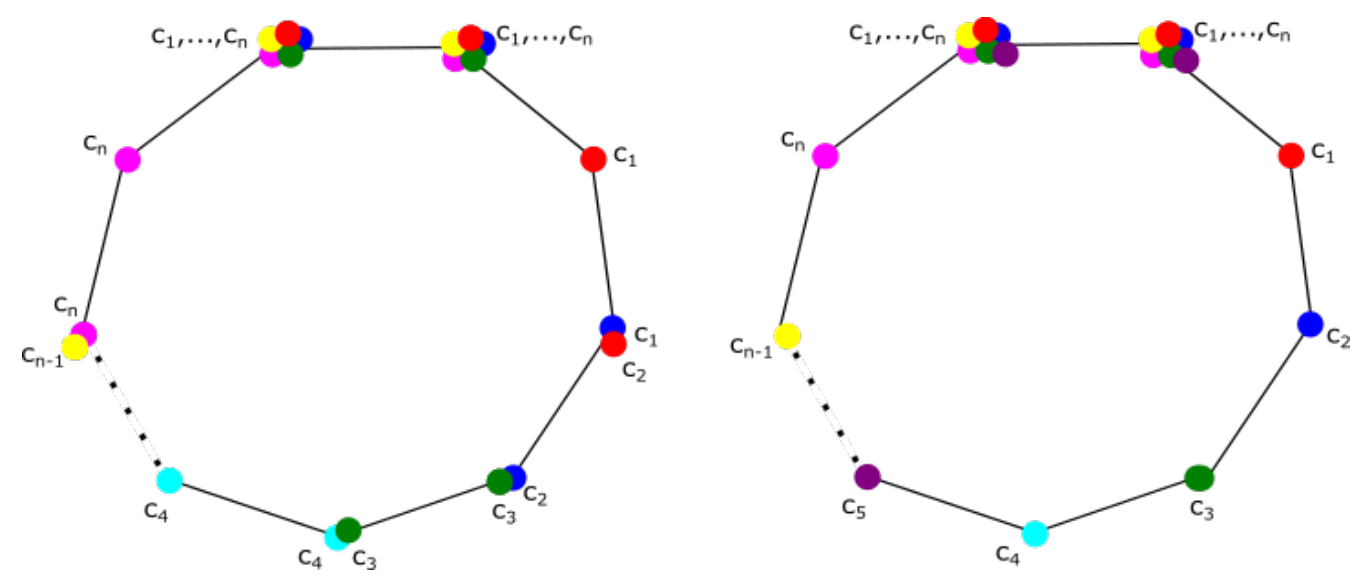

For the remainder of this subsection we work under the following additional hypotheses.

- $F$ has exactly one big petal, and

- the joint colouring of $F$ is of one of the forms described in Lemma 9.3.12 or Lemma 9.3.13

Lemma 9.3.14. If $F$ has an n-big joint-based 2-petal $P$ where $n \geq \sqrt{9.3 .14}(t)$ where the elements of this petal form a circuit with the basepoint $j$ of $P$, and for every $x \in X$ the shadow of $x$ on $P$ is parallel to an element of $P$ and no two shadows of elements of $X$ are parallel in $P$, then $M$ has a rank-t spike or an $M^{*}\left(K_{3, t}\right)$-minor. 
Proof. If $F$ has joint colouring of the first form in Lemma 9.3.12, when we contract all rim basepoints of $F$ we obtain a minor of $M$ that is a circuit with the property that there is an element of the circuit that is contained in a triangle with every other point of the circuit. This is a spike. Suppose $F^{\prime}$ has joint colouring of the second form from Lemma 9.3.12. Let $j$ be the rainbow joint, in other words $j$ is the basepoint of the $n$-big petal. Contracting $e$ gives a matching between two disjoint circuits and therefore $M^{\prime}$ has an $M^{*}\left(K_{3, n}\right)$-minor.

Lemma 9.3.15. If $F$ has an n-big joint-based 2-petal $P$ where the elements of $P$ are a series pair in $M$ with basepoint the basepoint of $P$, then $M$ has either a rank- $\frac{n}{2}$ spike or a double wheel with at least $\frac{n}{2}-1$ joints as a minor.

Proof. If $F$ has joint colouring of the second form from Lemma 9.3.12 then it is easy to see that $M$ has a rank- $n$ spike minor. Suppose $F$ has joint colouring of the first from of Lemma 9.3.12. Let $j$ be the joint of $P$ and let $P=\{a, b\}$. By a possible change of basis we can assume that $a$ is in $F(x)$ and $j$ is not for at least $\frac{|X|}{2}$ elements. We can delete all colours that do not have $a \in F(x)$ and $j \notin F(x)$, and find a minor $M^{\prime}$ of $M$ such that if $X^{\prime}=E\left(M^{\prime}\right) \cap X$ then the following holds.

1. $\left|X^{\prime}\right| \geq \frac{|X|}{2}$

2. the joint colouring of the canonical flower, $F^{\prime}$, of $F$ with respect to $X^{\prime}$ is of first form given in Lemma 9.3.12.

3. $F^{\prime}$ has a $\frac{|X|}{2}$-big petal $P$,

4. $P \cup B(P)$ is a triangle $a, b, j$ where $j$ is the basepoint of $P$, and

5. There is a $c \in\{a, b\}$ such that for any $x \in X^{\prime}$, the shadow of $x$ on $P$ is parallel to $c$

We may contract a rim element of $F^{\prime}$ in $M^{\prime}$ so that there is a point parallel to $j$ and delete any other resulting parallel elements. This can be seen to be a double wheel.

The proof of the following lemma is similar to that of the previous lemma and is omitted.

Lemma 9.3.16. If $F$ has an n-big rim-based 2-petal where the elements of this petal are a circuit, then $M$ has a rank-n spike or an $M^{*}\left(K_{3, n}\right)$-minor. 
Lemma 9.3.17. If $F$ has an $n$-big rim-based 2-petal $P$ where the elements are all parallel to the basepoint of $P$, then $M$ has a non graphic double wheel with rank $n$ or a rank-n spike as a minor.

Proof. Suppose the joint colouring of $F$ is of the second form given in Lemma 9.3.13. In this case $M$ can be represented by a matrix of the following form:

\begin{tabular}{|c|c|c|c|c|c|c|c|c|c|c|c|c|c|c|c|}
\hline & $r_{1}$ & $r_{2}$ & $r_{3}$ & $r_{4}$ & $r_{5}$ & $\ldots$ & $r_{n-1}$ & $r_{n}$ & $x_{1}$ & $x_{2}$ & $x_{3}$ & $x_{4}$ & $\ldots$ & $x_{n-3}$ & $x_{n-2}$ \\
\hline$j_{1}$ & 1 & 0 & 0 & 0 & 0 & & 0 & 1 & 1 & 1 & 1 & 1 & 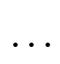 & 1 & 1 \\
\hline$j_{2}$ & 1 & 1 & 0 & 0 & 0 & $\cdots$ & 0 & 0 & 1 & 1 & 1 & 1 & .. & 1 & 1 \\
\hline$j_{3}$ & 0 & 1 & 1 & 0 & 0 & $\ldots$ & 0 & 0 & 1 & 0 & 0 & 0 & $\ldots$ & 0 & 0 \\
\hline$j_{4}$ & 0 & 0 & 1 & 1 & 0 & 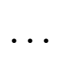 & 0 & 0 & 0 & 1 & 0 & 0 & .. & 0 & 0 \\
\hline$j_{5}$ & 0 & 0 & 0 & 1 & 1 & $\ldots$ & 0 & 0 & 0 & 0 & 1 & 0 & . & 0 & 0 \\
\hline$j_{6}$ & 0 & 0 & 0 & 0 & 1 & $\ldots$ & 0 & 0 & 0 & 0 & 0 & 1 & $\ldots$ & 0 & 0 \\
\hline 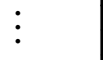 & $\vdots$ & $\vdots$ & $\vdots$ & $\vdots$ & $\cdot$ & $\vdots$ & $\vdots$ & $\vdots$ & $\vdots$ & $\vdots$ & $\vdots$ & & & & \\
\hline$j_{n-1}$ & 0 & 0 & 0 & 0 & 0 & $\ldots$ & 1 & 0 & 0 & 0 & 0 & 0 & $\ldots$ & 1 & 0 \\
\hline$j_{n}$ & 0 & 0 & 0 & 0 & 0 & $\cdots$ & 1 & 1 & 0 & 0 & 0 & 0 & $\ldots$ & 0 & 1 \\
\hline
\end{tabular}

It is then trivial to see that $M \backslash r_{1}$ is a non graphic double wheel. When the joint colouring of $F$ is of the first form from Lemma 9.3 .13 it is easy to see that $M$ has a spike minor.

Finally we need to consider the 3-petal case. The next lemma is essentially the same as Lemma 9.3.17.

Lemma 9.3.18. If $P$ is a 3-petal of $F$ in $M$ and the joints of $P$ are in $F(x)$ for every $x \in X$, then $M$ has a rank-n non graphic double wheel or a rank-n spike as a minor.

Lemma 9.3.19. There is a function $\sqrt{9.3 .19}$ such that if $n \geq \sqrt{9.3 .19}(t)$ the following holds. Suppose F has an n-big 3-petal P and is such that the following holds.

1. $M \mid(P \cup J(P))$ is a wheel,

2. the joints of $P$ are joints of this wheel

3. for every $x \in X$, the shadow of $x$ on $P$ is parallel to an element of $P$, and

4. no two shadows of elements of $X$ on $P$ are parallel. 
Then $M$ has a rank-t spike, a rank-t double wheel, a rank-t non graphic double wheel or $M^{*}\left(K_{3, t}\right)$ as a minor.

Proof. By reducing petals containing representatives of only one blocking element this case can be reduced to the case covered in Theorem 9.2.1.

Combining this with the results for blocking petals containing representatives of a large number of blocking elements we get the following theorem.

Theorem 9.3.20. There is a function $\sqrt{9.3 .20}$ such that the following holds. If $n \geq$ f9.3.20 $(t)$ and $F$ has an n-big petal then $M$ has a minor isomorphic to one of
i) $M^{*}\left(K_{3, t}\right)$
ii) a rank-t spike,
iii) a rank-t double wheel,
iv) a rank-t non graphic double wheel.

\section{Proof of Theorem 9.3 .1}

We now have all the tools we need to prove Theorem 9.3.1 which, for convenience, is restated below.

Theorem 9.3.1, There is a function $\int_{[9.3 .1}$ such that for all $t \geq 5$ the following holds. If $M$ is a binary matroid with a coindependent set $X$ such that

I) $M \backslash X$ has a maximal swirl-like pseudo-flower of order $n$ where $n \geq \sqrt{9.0 .1}(t)$,

II) every 3-separation of $M$ displayed by $F$ is blocked by an element of $X$,

III) the crossing graph of $X$ with respect to $F$ in $M$ is a complete graph,

$I V)$ there is no $x \in X$ that contains a representative in more than $k$ petals,

then $M$ has a minor isomorphic to one of the following:
i) a rank-t spike,
ii) a rank-t double wheel, 
iii) a rank-t non graphic double wheel,

iv) $M^{*}\left(K_{3, t}\right)$.

Proof. Let $n \geq \sqrt{9.3 .4}\left(\max \left\{\int_{9.3 .11}\left(\int_{9.3 .20}(t)\right), \int_{9.3 .20}(t)\right\}\right)$.

Since $n \geq \int \frac{9.3 .4}{(m)}$ where $\left.m=\max \left\{\int_{9.3 .11}\left(\int_{9.3 .20}(t)\right)\right), \int_{9.3 .20}(t)\right\}$, there is a minor $M_{1}$ of $M$ such that if $X_{1}=X \cap E\left(M_{1}\right)$ the following hold:

i) $M_{1} \backslash X_{1}$ has a swirl-like pseudo-flower $F_{1} \subseteq F$ of order $t$,

ii) any displayed 3-separation of $F_{1}$ in $M_{1}$ is blocked by some $x \in X_{1}$,

iii) the crossing graph of $X_{1}$ with respect to $F_{1}$ in $M_{1}$ is a complete graph,

iv) the joint colouring of $F_{1}$ in $M_{1}$ with respect to $X_{1}$ is such that every joint is coloured by either all colours in $\gamma\left(X_{1}\right)$ or exactly one colour in $\gamma\left(X_{1}\right)$, and

v) every proper petal in $F_{1}$ is either $\left|X_{1}\right|$-big or contains a representative of exactly one element of $X_{1}$.

Since $F_{1}$ has order at least $\int 9.3 .11\left(m^{\prime}\right)$ where $m^{\prime}=(\sqrt{9.3 .20}(t))$, either

- $M_{1}$ has a minor $M_{2}$ with coindepedent set $X_{2}$ such that the following holds:

i) $M_{2} \backslash X_{2}$ is a wheel,

ii) $X_{2}$ blocks all vertical 3-separations of $M_{2} \backslash X_{2}$,

iii) $\left|X_{2}\right| \geq m^{\prime}$, and

iv) the joint colouring of $M_{2} \backslash X_{2}$ has at least two rainbow joints and all colours distinguishable from the joint colouring

or

- $M$ has a minor isomorphic to one of the following.

i) a rank- $m^{\prime}$ double wheel,

ii) a rank- $m^{\prime}$ non graphic double wheel,

iii) $M^{*}\left(K_{3, m^{\prime}}\right)$,

iv) a rank- $m^{\prime}$ spike. 
Since $m, m^{\prime} \geq \sqrt{9.3 .20}(t)$ by Lemma 9.3.20 we now see that $M$ has a minor isomorphic to one of

i) $M^{*}\left(K_{3, t}\right)$,

ii) a rank- $t$ spike,

iii) a rank- $t$ double wheel,

iv) a rank- $t$ non graphic double wheel.

\subsection{Paths}

In this section we prove the following theorem.

Theorem 9.4.1. There is a function $\sqrt{9.4 .1}$ such that for all $t \geq 5$ the following holds. If $M$ is a binary matroid with a coindependent set $X$ such that

I) $M \backslash X$ has a maximal swirl-like pseudo-flower of order $n$ where $n \geq \sqrt{9.4 .1}(t)$,

II) every 3-separation of $M$ displayed by $F$ is blocked by an element of $X$,

III) the crossing graph of $X$ with respect to $F$ in $M$ is a path,

$I V)$ there is no $x \in X$ that contains a representative in more than $k$ petals,

then $M$ has a minor isomorphic to one of the following:

i) a rank-t spike,

ii) a rank-t double wheel,

iii) a rank-t circular ladder,

iv) a rank-t Möbius ladder,

v) $M\left(K_{3, t}\right)$,

vi) $M^{*}\left(K_{3, t}\right)$. 
In this section we work under the hypotheses of Theorem 9.4.1. That is we work under the following additional hypotheses:

- the crossing graph of $X$ with respect to $F$ in $M$ is a path,

- there is no $x \in X$ that contains a representative in more than $k$ petals.

Let $\gamma(X)=\left\{c_{1}, \ldots, c_{n^{\prime}}\right\}$ where $n^{\prime} \geq \frac{n}{k}$ and suppose the following holds:

1. $c_{i}$ crosses exactly $c_{i-1}$ and $c_{i+1}$, for $i \in\{2, \ldots, a-1\}$,

2. $c_{1}$ crosses exactly $c_{2}$, and

3. $c_{a}$ crosses exactly $c_{a-1}$.

We can relabel joints so that $c_{1}$ is assigned to $j_{1}$. To every colour $c_{i}$ assign a pair $\rho\left(c_{i}\right)=\left(j_{a}, j_{b}\right)$ where $j_{a}$ and $j_{b}$ are joints assigned $c_{i}$ and $j_{a}$ and $j_{b}$ have the property that no joint $j_{a^{\prime}}$ with $\left[j_{a^{\prime}}, j_{a}\right]_{j_{1}}$ is assigned colour $c_{i}$ and no $j b^{\prime}$ with $\left[j_{b}, j_{b^{\prime}}\right]_{j_{1}}$ is assigned colour $c_{i}$.

Definition 9.4.2. Let $C$ be a collection of colours with the property that the crossing graph of $C$ is a path. We say $\left\{c_{1}, \ldots, c_{d}\right\} \subseteq C$ is nested for $F$ in $M$ if, when the minimum element of $\rho\left(c_{i}\right)$ is less than the minimum element of $\rho\left(c_{j}\right)$, the maximum element of $\rho\left(c_{i}\right)$ is greater than the maximum element of $\rho\left(c_{j}\right)$ for $i, j \in\{1, \ldots, d\}$. We say that $F$ in $M \backslash X$ is partially blocked by a set $X$ in a nested way if $X$ is nested for $F$ in $M \backslash X$.

We say that a collection of colours $\left\{c_{1}, \ldots, c_{a}\right\} \subseteq C$ is shell-like for $F$ in $M$ if, when the minimum element of $\rho\left(c_{i}\right)$ is less then the minimum element of $\rho\left(c_{j}\right)$, the maximum element of $\rho\left(c_{i}\right)$ is at most the maximum element for $\rho\left(c_{j}\right)$. We say that $F$ in $M \backslash X$ is blocked by a set $X$ in a shell-like way if $X$ blocks all 3separations of $M \backslash X$ displayed by $F$ and $\gamma(X)$ is shell-like in $F$.

The definition above is a little indigestible so we give an example of a nested collection of colours and a shell-like collection of colours below. 


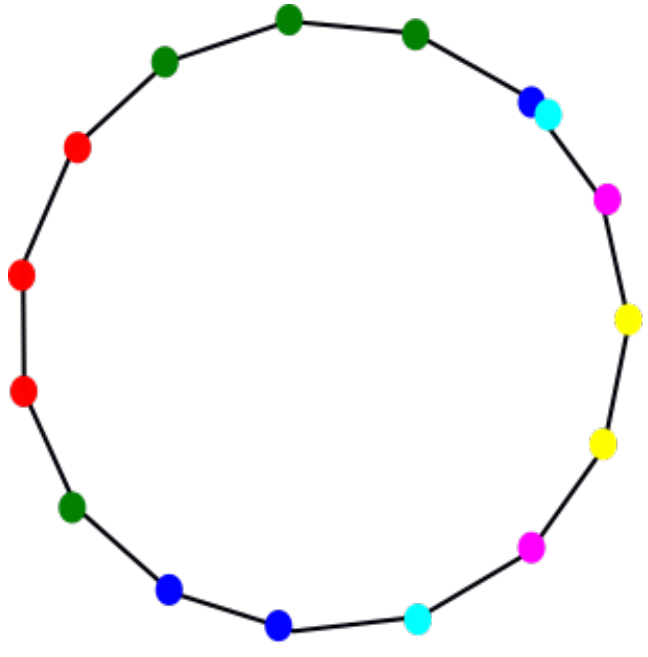

nested collection of colours

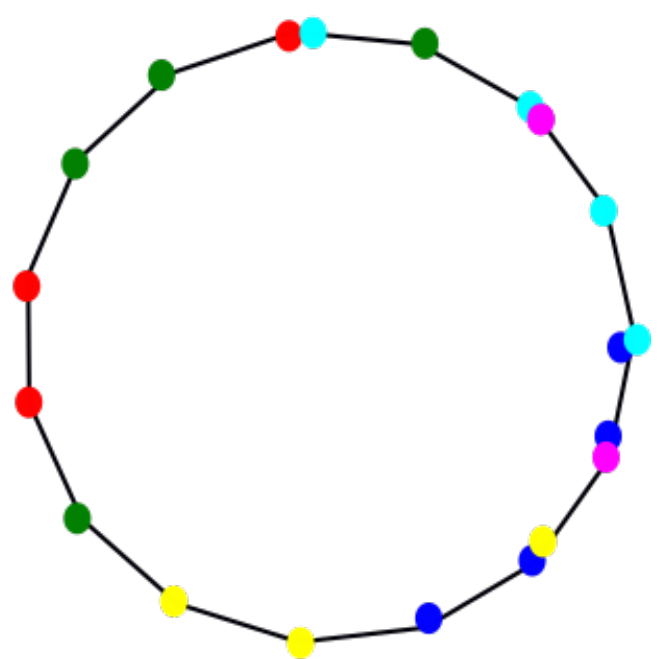

shell-like collection of colours

The following lemma is clear and thus the proof is omitted.

Lemma 9.4.3. There is a function $\sqrt{9.4 .3}$ such that if $n \geq \sqrt{9.4 .3}(t)$, then $F$ either contains a nested sequence of blocking elements of size at least $t$, or a set of $F$ of size at least $t$ that is blocked in a shell-like way.

We therefore add the following hypothesis.

- Either $X$ is shell-like for $F$ in $M$ or $X$ is nested for $F$ in $M$.

\subsubsection{Nested Blocking Elements}

Until stated otherwise we work under the following hypothesis.

- $X$ is nested for $F$ in $M$.

By removing petals we may assume that every element $x \in X$ contains a representative in exactly two petals. We therefore get the following lemma.

Lemma 9.4.4. There is a function $\sqrt{9.4 .4}$ such that if $n \geq \sqrt{9.4 .4}(t)$, then the following holds. Let $X^{\prime}=E\left(M^{\prime}\right) \cap X$. There is a minor $M^{\prime}$ of $M$ such that $\left|X^{\prime}\right| \geq t$, and $M^{\prime} \backslash X^{\prime}$ has a swirl-like pseudo-flower $F^{\prime}$, and that the joint colouring of $F^{\prime}$ with respect to $X^{\prime}$ is of one of the following forms. 


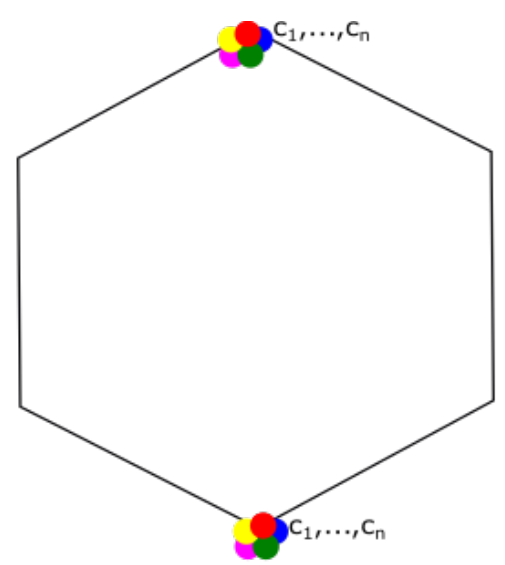

a)

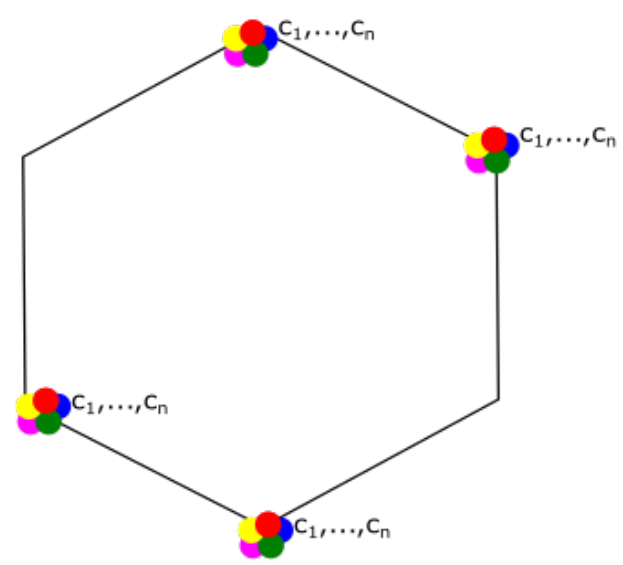

c)

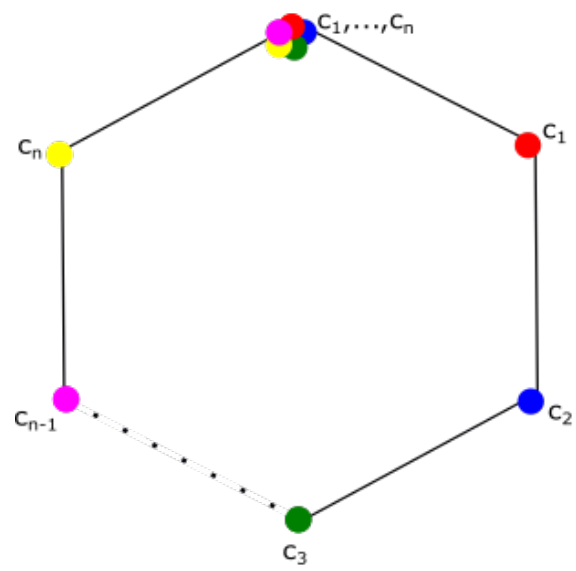

e)

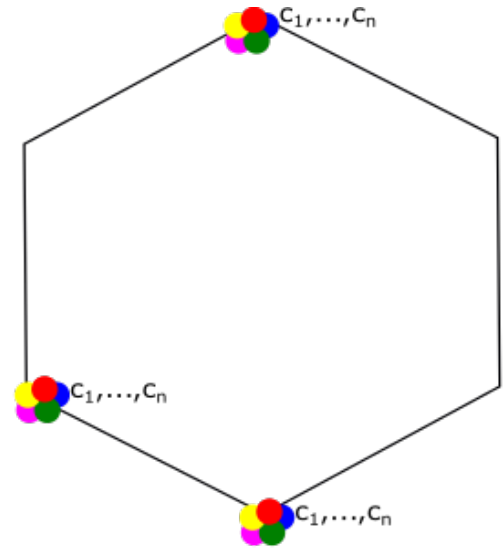

b)

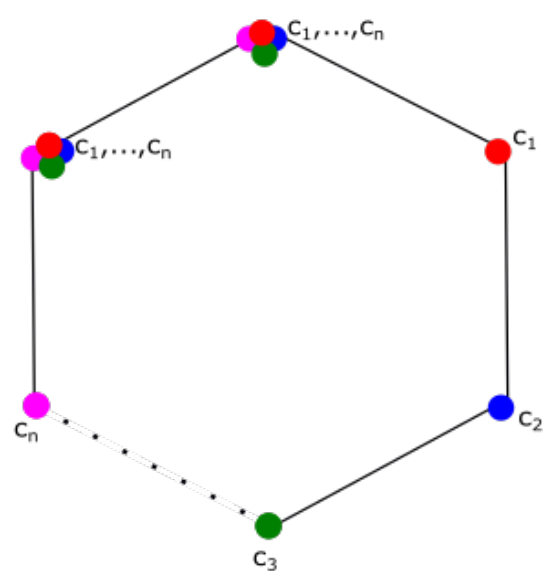

d)

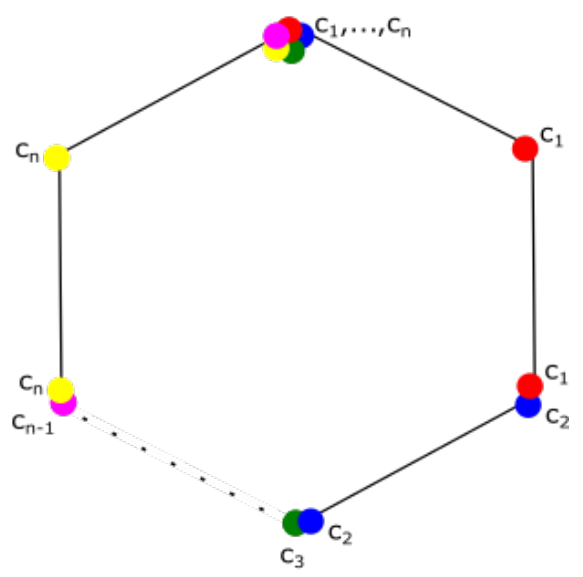

f) 


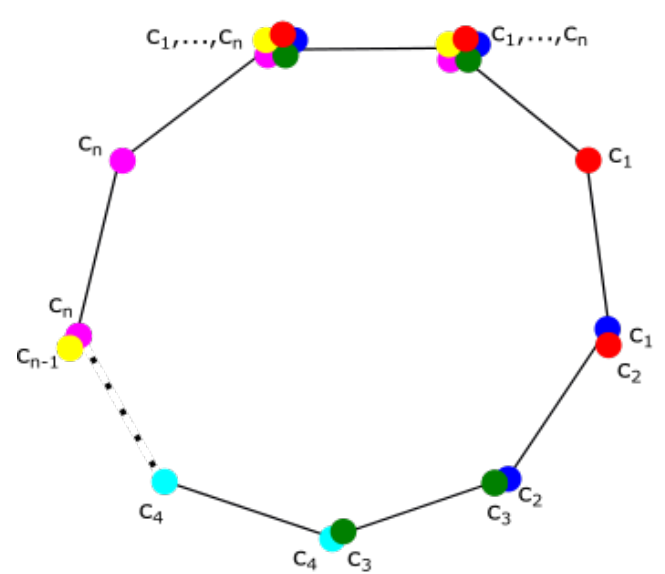

g)

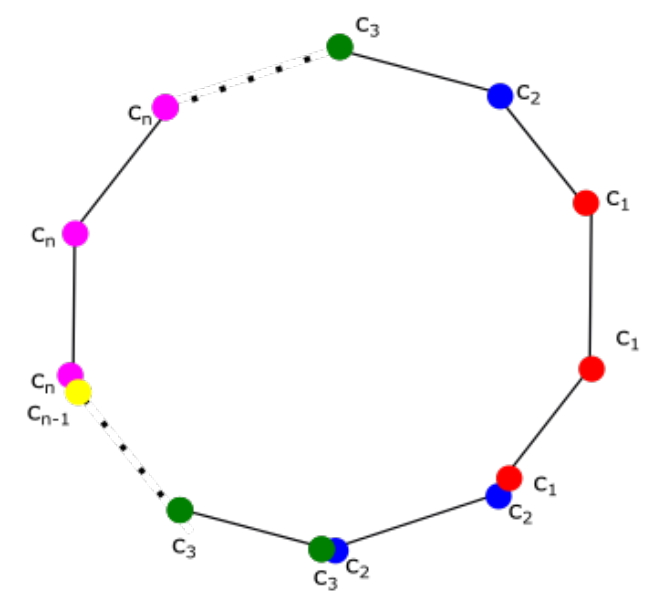

i)

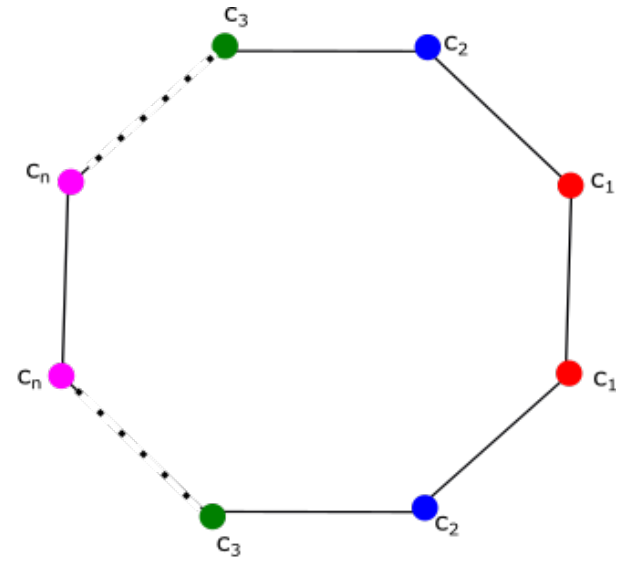

h)

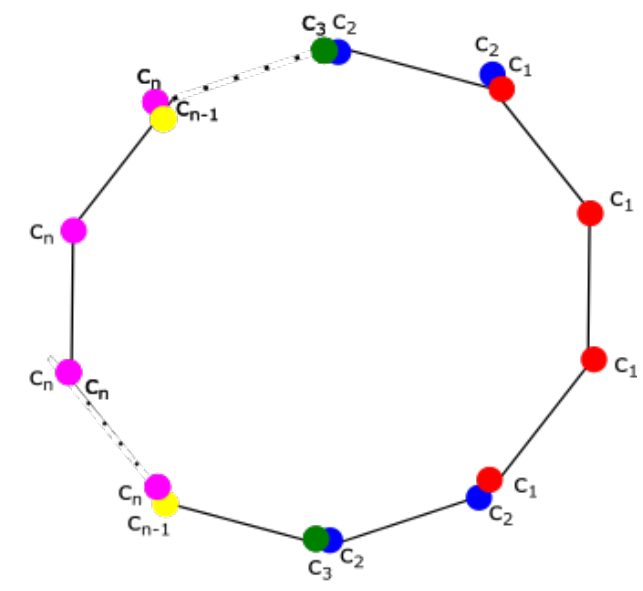

j)

Figure 9.1: Figure 9.4 .4

In the lemmas below we shall be referring to case $a$ ) in Figure 9.4.4 when we refer to case $a$ ) etc.

Lemma 9.4.5. There is a function $\sqrt{9.4 .5}$ such that if $n \geq \sqrt{9.4 .5}(t)$, then the following holds. If $F$ has joint colouring as in case a) then $M$ has an $M\left(K_{3, n}\right)$-minor.

Proof. If $F$ has joint colouring as in $a$ ) then either $j_{a}$ or $j_{b}$ (without loss of generality say $j_{a}$ ) is the basepoint of $t 2$-petals that each contain a representative of exactly one blocking element with colour in $c_{1}, \ldots, c_{t}$, and this blocking element is not parallel to the basepoint of any of these petals.

Consider the set $P_{1}, \ldots, P_{t}$ of petals with basepoint $j_{a}$. There is a minor $M_{1}$ of $M$ obtained by deleting $A \subseteq P_{i}$ and contracting $B \subseteq P_{i}$ such that $M_{1} \backslash X$ has a flower 
$F_{1}=\left(P_{1}, . ., P_{i-1}, P_{i}^{\prime}, P_{i+1}, \ldots, P_{t}\right)$, where $M_{1} \mid\left(P_{i}^{\prime} \cup j_{a}\right)$ is a triangle and $F\left(\gamma^{-1}\left(c_{i}\right)\right)$ contains an element of $P_{i}^{\prime}$ (note that $j_{a} \notin P_{i}^{\prime}$ ). For every $P_{i}$ with $i \in\{1, \ldots, t\}, M_{1} \mid P_{i}$ can be reduced in such a way. Let $M^{\prime}$ be a minor of $M$ that is such that $M^{\prime} \backslash X$ has swirl-like pseudo-flower $F^{\prime}=\left(P_{1}^{\prime}, \ldots, P_{t}^{\prime}, P_{t+1}, \ldots, P_{n}\right)$ in which every petal, $P_{i}^{\prime}$ has basepoint $j_{a}$ and is such that $M^{\prime} \mid\left(P_{i}^{\prime} \cup\left\{j_{a}\right\}\right)$ is a triangle and no shadow of $x$ on a petal is parallel to $j_{a}$ for $x \in \gamma^{-1}\left(\left\{c_{1}, \ldots, c_{t}\right\}\right)$.

Let $P_{1}^{\prime \prime}, \ldots, P_{t}^{\prime \prime}$ be the set of petals of $F$ with basepoint $j_{b}$ containing a representative of one of $\gamma^{-1}\left(\left\{c_{1}, \ldots, c_{t}\right\}\right)$. Suppose without loss of generality that $P_{i}^{\prime \prime}$ contains a representative of $\gamma^{-1}\left(c_{i}\right)=x_{i}$ for $i \in\{1, \ldots, t\}$. Let $M_{2}$ be a minor of $M^{\prime}$ that contains exactly one element, $a_{i}$ of $P_{i}^{\prime \prime}$ for $i \in\{1, \ldots, t\}$ and is such that the shadow of $x_{i}$ on $P_{i}^{\prime \prime}$ is parallel to $a_{i}$ and $a_{i}$ is parallel to $j_{b}$ in $M_{2}$. The minor $M_{2}$ of $M$ has a reduced representation given by the following matrix:

$$
\left(\begin{array}{ccccccccc}
1 & 1 & 0 & 0 & 0 & 0 & \ldots & 0 & 0 \\
0 & 0 & 1 & 1 & 0 & 0 & \ldots & 0 & 0 \\
0 & 0 & 0 & 0 & 1 & 1 & \ldots & 0 & 0 \\
\vdots & \vdots & \vdots & \vdots & \vdots & \vdots & \ddots & \vdots & \vdots \\
0 & 0 & 0 & 0 & 0 & 0 & \ldots & 1 & 1 \\
1 & 0 & 1 & 0 & 1 & 0 & \ldots & 1 & 0 \\
0 & 1 & 0 & 1 & 0 & 1 & \ldots & 0 & 1
\end{array}\right)
$$

Therefore $M$ has an $M\left(K_{3, t}\right)$-minor.

Lemma 9.4.6. There is a function $\sqrt{9.4 .6}$ such that if $n \geq \sqrt{9.4 .6}(t)$ then the following holds. If $F$ has joint colouring as in case $b$ ), then $M$ has a spike minor with rank $t$.

Proof. Let $P_{1}, \ldots, P_{n}$ be the rim-based 2-petals with joints $j_{b}$ and $j_{b+1}$. Suppose $P_{i}$ contains a representative of $\gamma^{-1}\left(c_{i}\right)$ and let this point be $p_{i}$. There is a minor of $M$ in which $p_{1}, \ldots, p_{i}$ form a circuit with $r_{b}$. When we contract $r_{b}$ it is easy to see a rank- $t$ spike minor of $M$.

Lemma 9.4.7. There is a function $\sqrt{[9.4 .7}$ such that if $n \geq \sqrt{9.4 .7}(t)$ then the following holds. If $F$ has a joint colouring as in case $c)$, then $M$ has an $M^{*}\left(K_{3, t}\right)$-minor.

Proof. This follows by Lemma 9.2.15 
Lemma 9.4.8. There is a function $\sqrt{9.4 .8}(t)$ such that if $n \geq \sqrt{9.4 .8}$ then the following holds. If $F$ has joint colouring as in d) or e) then $M$ has a spike minor with rank $t$, or an $M\left(K_{3, t}\right)$-minor.

Proof. These cases can be reduced to case $b$ ) or $a$ ) respectively by contracting rim elements.

Lemma 9.4.9. There is a function $\sqrt{9.4 .9}$ such that if $n \geq \sqrt{9.4 .9}(t)$ then the following holds. If $F$ has a joint colouring as in $f$ ) or $g$ ), then $M$ has a rank-t spike minor,

Proof. This follows from noting the that rim elements of $F$ form a circuit.

Lemma 9.4.10. There is a function $\sqrt{9.4 .10}$ such that if $n \geq \sqrt{9.4 .10}$ then the following holds. If $F$ has a joint colouring as in $h$ ), then $M$ has a rank-t clam as a minor.

Proof. This is immediate from the definition of a clam.

Lemma 9.4.11. There is a function $\sqrt{9.4 .11}$ such that if $n \geq \sqrt{9.4 .11}(t)$ then the following holds. If $F$ has a joint colouring as in $i)$, then $M$ has an $M^{*}\left(K_{3, t}\right)$-minor.

Proof. This is the same as the proof of Lemma 9.2.15.

Lemma 9.4.12. There is a function $\sqrt{9.4 .12}$ such that if $\geq \sqrt{9.4 .12}(t)$ then the following holds. If $F$ has a joint colouring as in $j$ ) then $M$ has a rank-t spike as a minor.

Proof. This can easily be reduced to case $f$ ).

The proof of the following lemma is now routine and left to the reader.

Lemma 9.4.13. There is a function $\sqrt{9.4 .13}$ such that the following holds. If $n \geq$ $\int 9.4 .13(t)$ and $X$ is nested, then $M$ has a minor isomorphic to one of the following matroids.

1. $M\left(K_{3, t}\right)$,

2. a rank-t spike,

3. $M^{*}\left(K_{3, t}\right)$,

4. a rank-t clam. 


\section{Shells}

We now discard the hypothesis that $F$ is blocked in a nested way and instead work under the following hypothesis.

- $F$ is blocked by $X$ in a shell-like way.

In what follows it is handy to use pictures a lot. In general in this subsection pictures of matroids will be pictures of petals in matroids. The points in the matroids come in several different types in the pictures. A square blue point represents a joint. If the petal we are drawing is a rim-based 2-petal or a 3-petal, $P_{i}$, then $j_{i}$ is the blue square on the left and $j_{i+1}$ is the blue square on the right. Black points are in $M$. When we block in a shell-like way, for any $P_{i}$ there is some $x_{i-1}, x_{i} \in X$ with $x_{i-1} \in \operatorname{cl}\left(P_{i-1} \cup P_{i}\right)$ and $x_{i} \in \operatorname{cl}\left(P_{i} \cup P_{i+1}\right)$. Consider the shadow $x_{i-1}^{\prime}$ of $x_{i-1}$ on $P_{i}$. If this is parallel to an element of $M$, then this is denoted by a green circle, otherwise it is denoted by a green triangle and this point is not in $M$. Consider the shadow $x_{i}^{\prime}$ of $x_{i}$ on $P_{i}$. If this is parallel to an element of $M$ then this is denoted by a red circle, otherwise it is denoted by a red triangle and this point is not in $M$. Notice that if we consider adjacent petals $P_{i}$ and $P_{i+1}$, there is a triangle containing the point of $P_{i}$ coloured red, and the point of $P_{i+1}$ coloured green, and blocking element $x_{i}$.

The following lemma follows by concatenating petals.

Lemma 9.4.14. There is a function $\sqrt{9.4 .14}$ such that the following holds. If $n \geq$ $9.4 .14(t)$, then there is a minor $M^{\prime}$ of $M$ with coindependent set $X^{\prime}$ such that $M^{\prime} \backslash X^{\prime}$ has a swirl-like pseudo-flower $F^{\prime}$ of order at least $t$ and the following hold.

i) $F^{\prime}$ is blocked by $X^{\prime}$ in a shell-like way,

ii) every petal if $F^{\prime}$ is a 3-petal,

iii) every $x \in X^{\prime}$ has a representative in exactly two petals and these petals are adjacent,

iv) if $x, y \in X$ cross then there is a petal, $P_{i}$, containing a representative of $x$ and a representative of $y$. Moreover, if $x$ contains a representative in $P_{i-1}$ and $y$ contains a representative in $P_{i+1}$ then there is no 2-separation $(A, B)$ in $M \mid\left(P_{i} \cup J\left(P_{i}\right)\right)$ such that $\left\{x, j_{i}\right\} \in A$ and $\left\{y, j_{i+1}\right\} \in B$. 
Until otherwise stated we work under the following hypotheses.

- $F^{\prime}$ is blocked by $X^{\prime}$ in a shell-like way,

- every petal if $F^{\prime}$ is a 3-petal,

- every $x \in X^{\prime}$ has a representative in exactly two petals and these petals are adjacent,

- if $x, y \in X$ cross then there is a petal, $P_{i}$, containing a representative of $x$ and a representative of $y$ and if $x$ contains a representative in $P_{i-1}$ and $y$ contains a representative in $P_{i+1}$ then there is no 2-separation $(A, B)$ in $M \mid\left(P_{i} \cup J\left(P_{i}\right)\right.$ such that $\left\{x, j_{i}\right\} \in A$ and $\left\{y, j_{i+1}\right\} \in B$.

Lemma 9.4.15. Let $P_{i}$ be a 3-petal of $F$ containing representatives of blocking elements $x_{1}$ and $x_{2}$. Suppose that $x_{1}$ contains a representative in $P_{i-1}$ and $x_{2}$ contains a representative in $P_{i+1}$. Then $M$ has a minor $M^{\prime}$ such that $X \subseteq M^{\prime}$ and $M^{\prime} \backslash X$ has a flower $F=\left(P_{1}, \ldots, P_{i-1}, P_{i}^{\prime}, P_{i+1}, \ldots, P_{n}\right)$ where $M^{\prime} \mid P_{i}^{\prime}$ is of one of the following forms:

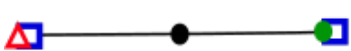

a)

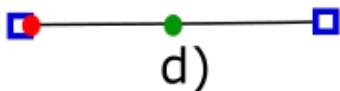

d)

g)

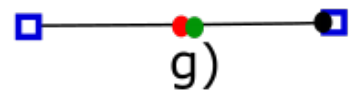

$\Delta$

b)

b)

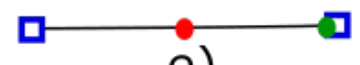

e)

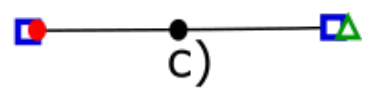

[-

f)

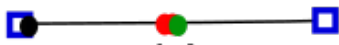

h) 


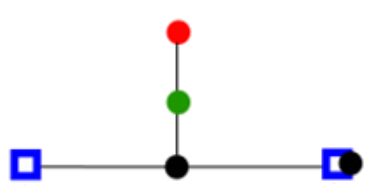

i)

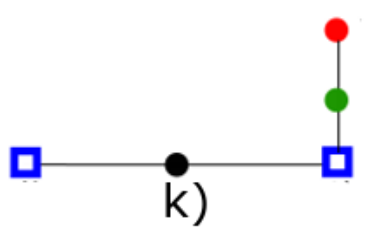

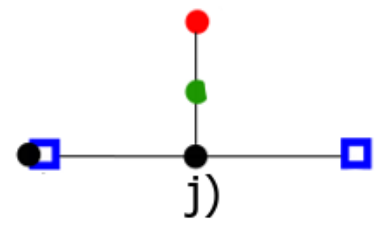

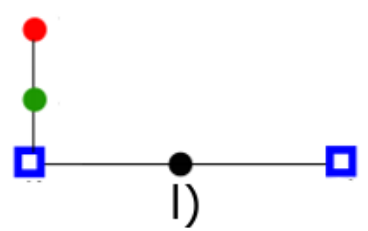

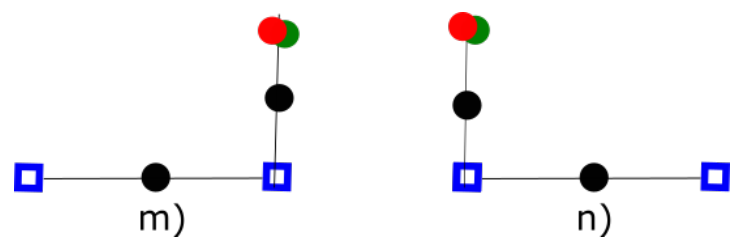

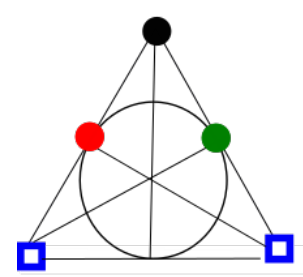

o)

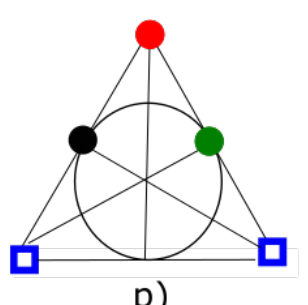

p)

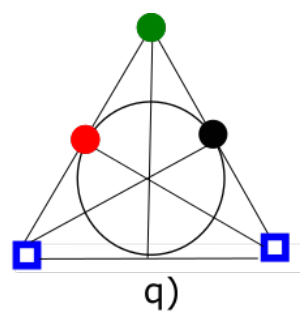

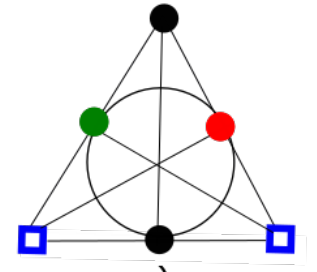

r)

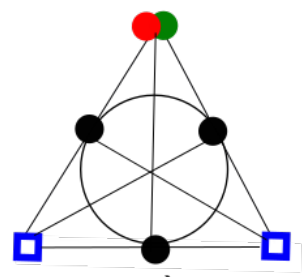

s) 


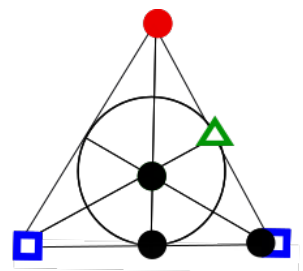

t)

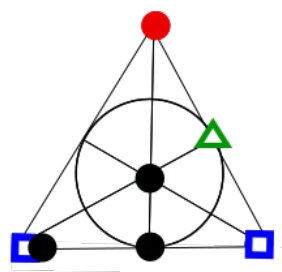

w)

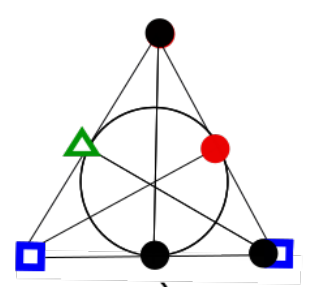

z)

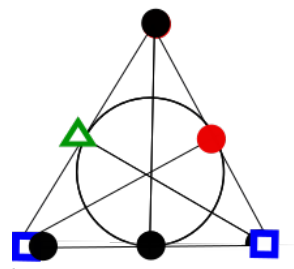

C)

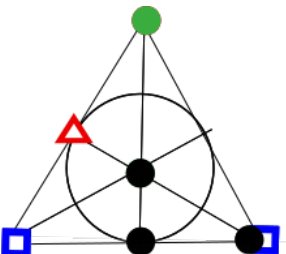

u)

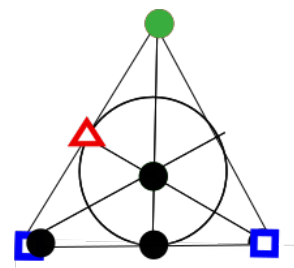

$\mathrm{x})$

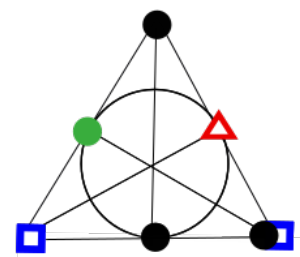

A)

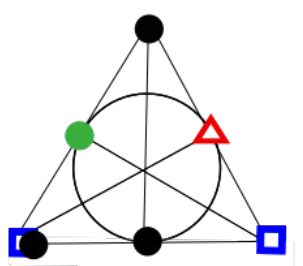

D)
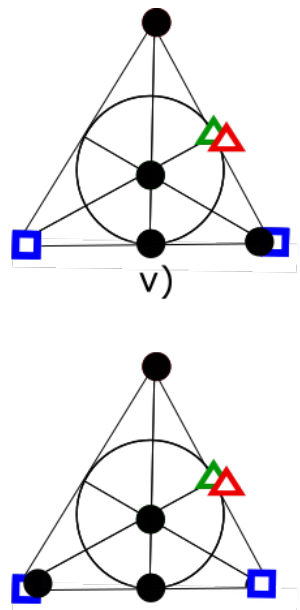

y)

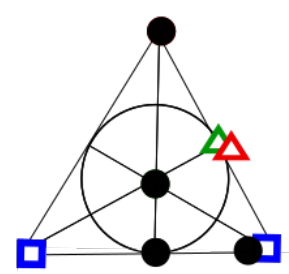

B)

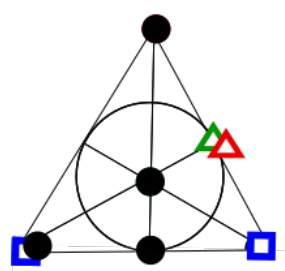

E)

Proof. Let $x_{i}^{\prime}$ be the shadow of $x_{i}$ on $P$. Consider the elements of $F\left(x_{1}^{\prime}\right)$ and $F\left(x_{2}^{\prime}\right)$. Suppose $j_{i} \in F\left(x_{1}^{\prime}\right)$. If $j_{i-1} \in F\left(x_{2}^{\prime}\right)$ there is a minor $M^{\prime}$ of $M$ obtained by deleting $A \subseteq P_{i}$ and contracting $B \subseteq P_{i}$ such that $M^{\prime} \backslash X$ has a flower $F=\left(P_{1}, . ., P_{i-1}, P_{i}^{\prime}, P_{i+1}, \ldots, P_{n}\right)$ and $M \mid P_{i}^{\prime}$ is of the one of the following forms: $a), b), c), d), e), f), g)$ or $h)$. If $j_{i-1} \notin F\left(x_{2}^{\prime}\right)$ and $j_{i} \in F\left(x_{1}^{\prime}\right)$ or $j_{i-1} \in F\left(x_{2}^{\prime}\right)$ and $j_{i} \notin F\left(x_{1}^{\prime}\right)$, then by Lemma 2.1 .16 there is a minor $M^{\prime}$ of $M$ obtained by deleting $A \subseteq P_{i}$ and contracting $B \subseteq P_{i}$ such that $M^{\prime} \backslash X$ has a flower $F=$ $\left(P_{1}, . ., P_{i-1}, P_{i}^{\prime}, P_{i+1}, \ldots, P_{n}\right)$ and $M \mid P_{i}^{\prime}$ is of the one of the following forms: 

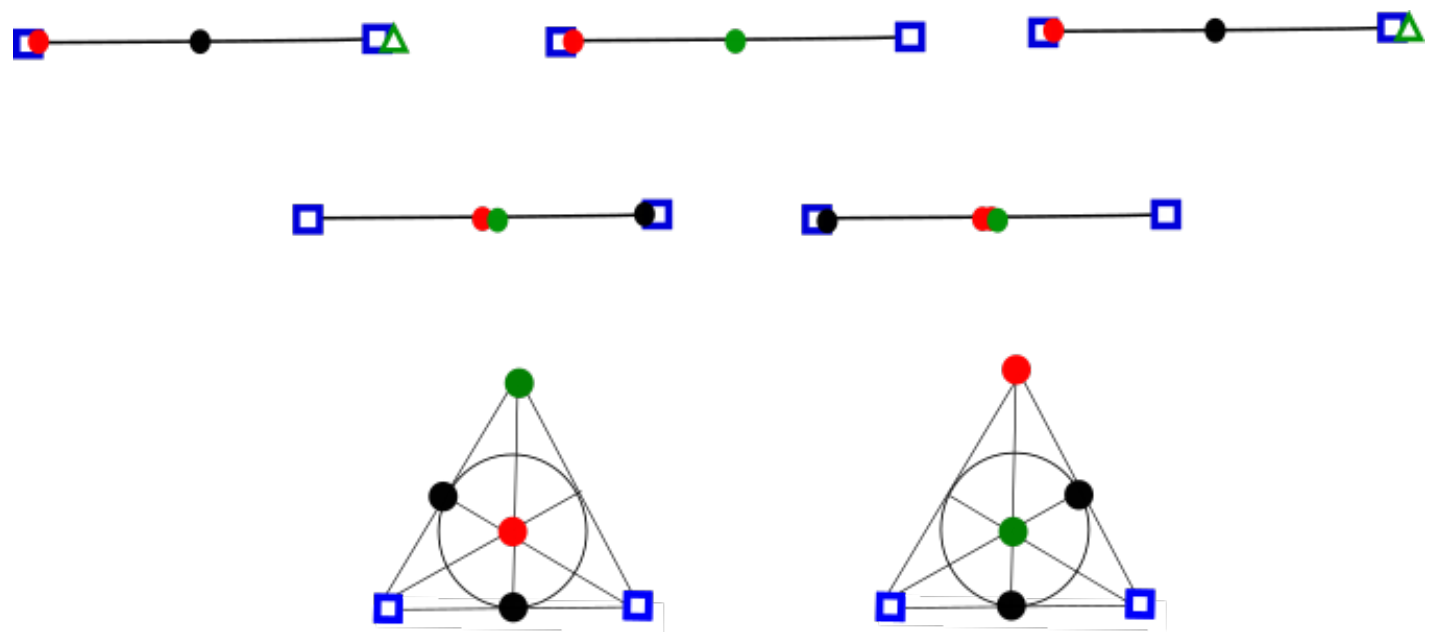

So suppose that $F\left(x_{1}^{\prime}\right) \cap J\left(P_{i}\right) \in\left\{j_{i-1}, \emptyset\right\}$ and $F\left(x_{2}^{\prime}\right) \cap J\left(P_{i}\right) \in\left\{j_{i}, \emptyset\right\}$. Consider the non-joint elements of $F\left(x_{1}^{\prime}\right)$ and $F\left(x_{2}^{\prime}\right)$. These must exist as otherwise $x_{1}$ and $x_{2}$ would not cross. There must be some pair of elements, $r$ and $g$, with $g \in F\left(x_{1}^{\prime}\right)$ and $r \in F\left(x_{2}^{\prime}\right)$ that are such that there is no 2-separation of $P_{i}$ separating $r$ and $g$. We may then apply Lemma 8.3 .6 to elements $r, g, j_{i-1}, j_{i}$. The shadow of $x_{1}$ on $P$ is then either parallel to $g$ or in the closure of $g$ and $j_{i-1}$, and the shadow of $x_{2}$ on $P$ is then either parallel to $r$ or in the closure of $r$ and $j_{i}$. A case analysis of this then gives one of the situations described in the statement of the lemma.

We can consider a minor $M^{\prime}$ of $M$ obtained by "composing adjacent petals", where composition is described as follows. Let $P_{i}$ and $P_{i+1}$ be two adjacent petals of $F$. Suppose $P_{i}$ is a 3-petal of $F$ containing representatives of blocking elements $x_{1}$ and $x_{2}$. Suppose that $x_{1}$ contains a representative in some petal $P_{a}$, and $x_{2}$ containsa representative in $P_{i+1}$, with none of $P_{a}, P_{i}, P_{i+1}$ equal. Further suppose that $\left[P_{a}, P_{i}, P_{i+1}\right]_{P_{1}}$. Suppose $P_{i+1}$ is a 3 -petal of $F$ containing representatives of blocking elements $x_{2}$ and $x_{3}$. Suppose that $x_{3}$ contains a representative in some petal $P_{b}$ and we know $x_{2}$ contains a representative in $P_{i}$. We compose $P_{i}$ and $P_{i+1}$ by considering a minor of $M^{\prime}$ of $M$ with $M^{\prime} \backslash X$ having swirl-like pseudo-flower $F^{\prime}=\left(P_{1}, \ldots, P_{i-1}, P_{i}^{\prime}, P_{i+2}, P_{n}\right)$ where $M^{\prime} \mid P_{i}^{\prime}$ is a minor of $M \mid\left(P_{i} \cup P_{i+1} \cup\left\{x_{2}\right\}\right)$ and is of one of the forms described in Lemma 9.4.15 (We know this will be possible as there is no 2-separation with $x_{1}^{\prime}$ on one side and $x_{3}^{\prime}$ on the other). Therefore, for every pair of arrangements from Lemma 9.4.15, we have an arrangement from Lemma 9.4 .15 to send this pair to in the composition. There may be sev- 
eral possible choices for composition of two petals but we fix one of these to be the composition. This gives a multiplication table whose entries come from a,b,c,d,e,f,g,h,i,j,k,l,m,n,o,p,q,r,s,t,u,v,w,x,y,z,A,B,C. We use $P_{i} \circ P_{i+1}$ to denote concatenation of $P_{i}$ and $P_{i+1}$, and if we see $P_{i} \circ P_{i+1} \circ P_{i+2}$ we concatenate left to right, in other words $\left(P_{i} \circ P_{i+1}\right) \circ P_{i+2}$

The proof of the following lemma is courtesy of Jim Geelen.

Lemma 9.4.16. Let $S$ be a string taking entries from a finite alphabet $A$, let $\mathscr{S}$ be the set of all substrings of $S$, and let $\phi$ be a function taking elements of $\mathscr{S}$ to elements of $A$. There is some function $\sqrt{9.4 .16}$ such that if $S$ is a sequence of elements from $A$ of length at least $\sqrt{9.4 .16}(t)$, then there is an $a \in A$ such that the following holds. There is a substring $a_{i_{1}}, a_{i_{2}}, \ldots, a_{i_{n+1}}$ of $S$ such that $\phi\left(a_{i_{k}}, a_{i_{k}+1}, \ldots, a_{i_{k+1}-1}\right)=$ a for $i \in\{1, \ldots, n\}$.

Proof. Suppose $S=\left(a_{1}, \ldots, a_{n}\right)$ and for each $i \in\{1, \ldots, n\}$ construct a vector $b_{i} \in$ $\mathbb{Z}^{A}$ where, for each $a \in A$, we let $b_{i}(a)$ denote the longest sequence of consecutive substrings of $S$ starting at $x_{i}$ that each have value $a$. Note that no two of the vectors $b_{1}, \ldots, b_{n}$ are the same, so if $n>t^{|A|}$ then there is some $b_{i}(a)$ that is at least $t+1$.

From this we immediately get the following lemma.

Lemma 9.4.17. There is a function 9.4 .17 such that the following holds. Suppose $n \geq \sqrt{9.4 .17}(t)$. Then there is some $\alpha \in$ $\{a, b, c, d, e, f, g, h, i, j, k, l, m, n, o, p, q, r, s, t, u, v, w, x, y, z, A, B, C, D, E\}$ such that there is some subset $P_{i_{1}}, \ldots, P_{i_{t+1}}$ of petals of $F$ such that $P_{i_{k}} \circ P_{i_{k}+1} \circ \ldots \circ P_{i_{k+1}-1}=a$ for $k \in\{1, \ldots, n\}$.

We therefore add the following hypothesis.

- All petals of $F-\left\{P_{1}, P_{n}\right\}$ have the same form and that form is one of $\{a, b, c, d, e, f, g, h, i, j, k, l, m, n, o, p, q, r, s, t, u, v, w, x, y, z, A, B, C, D, E\}$.

Therefore we are only interested in composing a petal with another of the same type, so fortunately we can restrict our attention to the diagonal entries of the multiplication table for composition of petals. 
The diagonal entries of the multiplication table are as follows where $\circ$ is the symbol for composition:

$$
\begin{array}{cccc}
a \circ a=a & b \circ b=e & c \circ c=c & d \circ d=e \\
e \circ e=d & f \circ f=d & g \circ g=r & h \circ h=r \\
i \circ i=m & j \circ j=m & k \circ k=r & l \circ l=r \\
m \circ m=s & n \circ n=s & o \circ o=j & p \circ p=e \\
q \circ q=d & r \circ r=r & s \circ s=s & t \circ t=v \\
u \circ u=y & v \circ v=v & w \circ w=v & x \circ x=y \\
y \circ y=y & z \circ z=z & A \circ A=A & B \circ B=v \\
C \circ C=C & D \circ D=D & E \circ E=y &
\end{array}
$$

Lemma 9.4.18. There is a function $\sqrt{9.4 .18}$ such that the following holds. If $n \geq \sqrt{9.4 .18}(t)$ and all petals of $F$ are of the same form and this is one of forms $a, b, c, d, e, f, g, h, i, j, k, l, m, n, o, p, q, r, s, t, u, v, w, x, y, z, A, B, C, D, E$, then $M$ has a minor $M^{\prime}$ such that the following hold.

1. $M^{\prime}$ has a coindependent set $X^{\prime}$ such that $M \backslash X^{\prime}$ has a maximal swirl-like pseudo-flower $F^{\prime}$ of order at least $t$,

2. Every 3-separation of $M^{\prime}$ displayed by $F^{\prime}$ is blocked by an element of $X^{\prime}$,

3. $X^{\prime}$ blocks $F$ in a shell-like way,

4. every petal of $F$ is of form a, or every petal of $F$ is of form $c$, or every petal of $F$ is ofform $r$ or every petal of $F$ is of form $s$.

Proof. Many of the cases can be easily seen from the composition rules above. We give details on the cases that cannot.

If $F$ in $M \backslash X$ has 6 consecutive petals $P_{1}, \ldots, P_{6}$ of form $v$ ), then there is a minor $M^{\prime}$ of $M$ such that $M^{\prime} \backslash\left(X-\left\{x_{1}, x_{2}, x_{4}, x_{5}\right\}\right)$ has a flower $F^{\prime}=\left(P, P^{\prime}, P_{7}, \ldots, P_{n}\right)$ such that $M^{\prime}\left|\left(P_{7} \cup \cdots \cup P_{n}\right)=M\right|\left(P_{7} \cup \cdots \cup P_{n}\right)$ and $P, P^{\prime}$ both have form $\left.l\right)$. These two petals can then be composed to give a minor $M^{\prime \prime}$ of $M$ such that $M^{\prime \prime} \backslash(X-$ $\left.\left\{x_{1}, \ldots, x_{5}\right\}\right)$ has a flower $F^{\prime \prime}=P^{\prime \prime}, P_{7}, \ldots, P_{n}$ such that $M^{\prime}\left|\left(P_{7} \cup \cdots \cup P_{n}\right)=M\right|\left(P_{7} \cup\right.$ $\left.\cdots \cup P_{n}\right)$ and $P^{\prime \prime}$ has form $r$ ). We can do a similar thing with petals of form $y$ ).

If $F$ in $M \backslash X$ has 3 consecutive petals $P_{1}, P_{2}, P_{3}$ of form $z$ ), then there is a minor $M^{\prime}$ of $M$ such that $M^{\prime} \backslash\left(X-\left\{x_{1}, x_{2}\right\}\right)$ has a flower $F^{\prime}=P^{\prime}, P_{4}, \ldots, P_{n}$ such that 
$M^{\prime}\left|\left(P_{4} \cup \cdots \cup P_{n}\right)=M\right|\left(P_{4} \cup \cdots \cup P_{n}\right)$ and $P^{\prime}$ has form $\left.r\right)$. We can do a similar thing for petals of form $A), a$ ) and $D$ ).

If $F$ in $M \backslash X$ has 3 consecutive petals $P_{1}, P_{2}, P_{3}$ of form $d$ ) we can compose $P_{1}$ and $P_{2}$ to get a petal of form $e$ ) followed by a petal of form $d$ ). The same things applies if $F$ has 3 consecutive petals of form $e$ ).

If $F$ in $M \backslash X$ has 2 consecutive petals $P_{1}, P_{2}$ where $P_{1}$ has form $d$ ) and $P_{2}$ has form $d)$, then there is a minor $M^{\prime}$ of $M$ such that $M^{\prime} \backslash\left(X-\left\{x_{1}\right\}\right)$ has a flower $F^{\prime}=P^{\prime}, P_{3}, \ldots, P_{n}$ such that $M^{\prime}\left|\left(P_{3} \cup \cdots \cup P_{n}\right)=M\right|\left(P_{3} \cup \cdots \cup P_{n}\right)$ and $P^{\prime}$ has form $s)$. We can do the same thing when $P_{1}$ has form $e$ ) and $P_{2}$ form $d$ ).

The following lemma is clear.

Lemma 9.4.19. If the petals of $F$ are all of type a) or all of type $c$ ) then $M$ is graphic.

Lemma 9.4.20. There is a function $\sqrt{9.4 .20}$ such that if $n \geq \sqrt{9.4 .20}(t)$, then the following holds. If the petals of $F$ are all of type $p$ ) or q) then M has a rank-t circular ladder as a minor.

Proof. If all petals are of type $p$ we have the matroid below where $g_{1}, r_{1}, g_{2}, r_{2}, \ldots, g_{n}, r_{n}$ form a circuit.

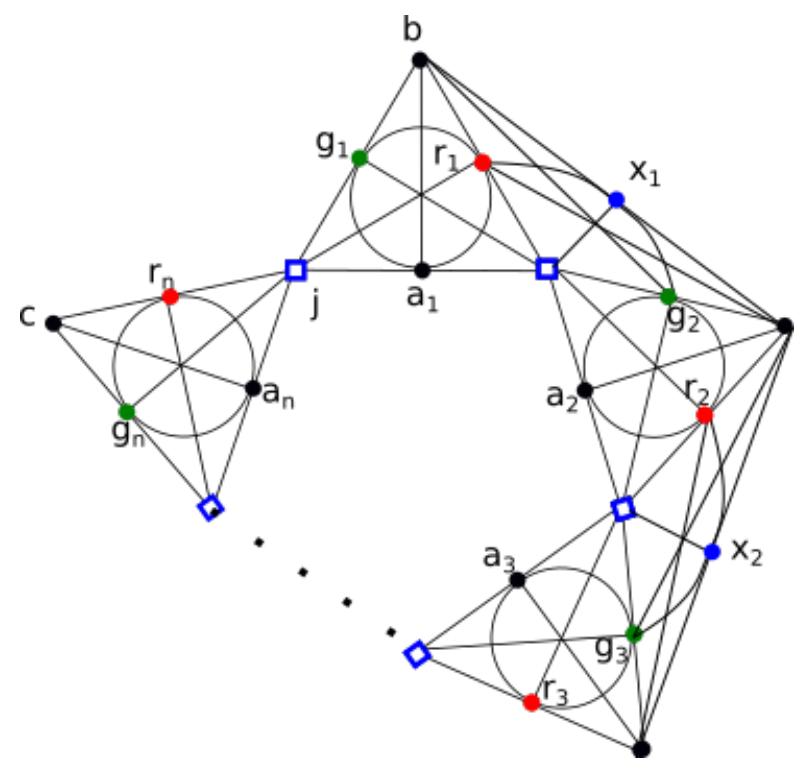


We can then see that $M \mid\left\{g_{1}, r_{1}, g_{2}, r_{2}, \ldots, g_{n}, r_{n}, x_{1}, x_{2}, \ldots, x_{n-1}, b, c, j\right\}$ is a circular ladder. The same proof holds when the petals are of type $q$.

This means that the proof of the following lemma is routine. In this lemma we have discarded all hypotheses.

Lemma 9.4.21. There is a function $f_{9.4 .21}$ such that if $M$ is a binary matroid with coindependent set $X$ such that the following hold:

i) $M \backslash X$ has a maximal swirl-like pseudo-flower $F$,

ii) $F$ is blocked by $X$ and the crossing graph of $X$ is a path,

iii) there is a consecutive set of petals of $F$ of size at least $[9.4 .21(n)$ where the elements in this section block $F$ in a shell-like way,

then there is a minor of $M$ that is either an n-rung circular ladder, an n-rung Möbius ladder, a double wheel or $M\left(K_{4, n}\right)$.

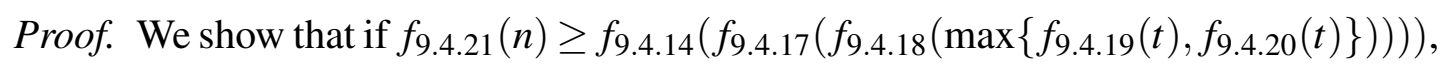
then the result follows. By Lemma 9.4.14 there is a minor $M_{1}$ of $M$ with coindependent set $X_{1}$ such that $M_{1} \backslash X_{1}$ has a swirl-like pseudo-flower $F_{1}$ of order at least $\left(\int_{9.4 .17}\left(\int \frac{9.4 .18}{}\left(\max \left\{\int_{9.4 .19}(t), \int_{9.4 .20}(t)\right\}\right)\right)\right.$ and the following hold.

i) $F_{1}$ is blocked by $X_{1}$ in a shell-like way,

ii) every petal of $F_{1}$ is a 3-petal,

iii) every $x \in X_{1}$ has a representative in exactly two petals and these petals are adjacent,

iv) if $x, y \in X_{1}$ cross then there is a petal, $P_{i}$, of $F_{1}$ containing a representative of $x$ and a representative of $y$, and if $x$ contains a representative in $P_{i-1}$ and $y$ contains a representative in $P_{i+1}$, then there is no 2-separation $(A, B)$ in $M \mid\left(P_{i} \cup J\left(P_{i}\right)\right)$ such that $\left\{x, j_{i}\right\} \in A$ and $\left\{y, j_{i+1}\right\} \in B$.

By Lemma 9.4.17 there is some consecutive subset of petals of $F_{1}$ that can be partitioned into $f_{9.4 .18}\left(\max \left\{\int_{9.4 .19}(t), f_{9.4 .20}(t)\right\}\right)$ parts such that if $P_{i}$ and $P_{j}$ are petals of $F^{\prime}$ contained in the same part then so is $P_{k}$ for all petals $P_{k}$ such that $\left[P_{i}, P_{k}, P_{j}\right]_{P_{1}}$, and all parts concatenate to give the same thing. 
By the multiplication table for concatenation there is a minor $M_{2}$ of $M_{1}$ with coindependent set $X_{2}$ such that $M_{2} \backslash X_{2}$ has a swirl-like pseudo-flower $F_{2}$ of order at least $\left.\max \left\{f \sqrt{9.4 .19}(t), f_{9.4 .20}(t)\right\}\right)$ and the following hold.

i) $F_{2}$ is blocked by $X_{2}$ in a shell-like way,

ii) every petal if $F_{2}$ is a 3-petal,

iii) every $x \in X_{2}$ has a representative in exactly two petals and these petals are adjacent,

iv) if $x, y \in X_{2}$ cross then there is a petal, $P_{i}$ of $F_{2}$ containing a representative of $x$ and a representative of $y$, and if $x$ contains a representative in $P_{i-1}$ and $y$ contains a representative in $P_{i+1}$, then there is no 2-separation $(A, B)$ in $M \mid\left(P_{i} \cup J\left(P_{i}\right)\right)$ such that $\left\{x, j_{i}\right\} \in A$ and $\left\{y, j_{i+1}\right\} \in B$.

v) all petals in $F_{2}$ are of the same form and that form is one of $\left.\left.\left.a\right), c\right), r\right), s$ ).

Since the order of $F_{3}$ is at least $\left.\max \{\sqrt{9.4 .19}(t), \sqrt{9.4 .20}(t)\}\right)$, the result now follows from Lemma 9.4.19 and Lemma 9.4.20.

The proof of Theorem 9.4.1 is now routine and is omitted.

\subsection{Proof of Theorem 9.0.1}

We now have all the pieces of the jigsaw that is the proof of Theorem 9.0.1 and all that remains is to put them together.

Theorem 9.0.1. There is a function $\sqrt{9.0 .1}$ such that for all $t \geq 5$ the following hold. If $M$ is a binary matroid with a coindependent set $X$ such that $M \backslash X$ has a maximal swirl-like pseudo-flower of order $n$ where $n \geq \sqrt{9.0 .1}(t)$ and every 3-separation of $M$ displayed by $F$ is blocked by an element of $X$, then $M$ has minor isomorphic to one of the following:
i) a rank-t circular ladder,
ii) a rank-t Möbius ladder,
iii) a rank-t spike, 
iv) a rank-t double wheel,

v) a rank-t non graphic double wheel,

vi) $N\left(K_{3, t}\right)$,

vii) $M\left(K_{4, t}\right)$,

viii) a rank-t clam,

ix) $M^{*}\left(K_{3, t}\right)$ blocked in a path-like way

Proof. Suppose $n \geq \sqrt{8.2 .6}(\max \{\sqrt{9.1 .3}(t), \sqrt{9.2 .1}(t), \sqrt{9.3 .1}(t), \sqrt{9.4 .1}(t)\}, \sqrt{9.1 .3}(t))$. By Theorem 8.2.6 either there is some $x \in X$ that blocks at least $\sqrt{9.1 .3}(t)$ displayed separations of $M$ or there is a minor $M^{\prime}$ of $M$ with coindependent set $X^{\prime}=X \cap E\left(M^{\prime}\right)$ such that the following hold.

1. $M^{\prime} \backslash X^{\prime}$ has a swirl-like pseudo-flower $F^{\prime}$,

2. every 3-separation of $M^{\prime}$ displayed by $F^{\prime}$ is blocked by some element $x \in X^{\prime}$,

3. the crossing graph of $X^{\prime}$ with respect to $F^{\prime}$ in $M^{\prime}$ is either a star, a path or a complete graph on at least $\max \left\{\int_{9.2 .1}(t), \int_{9.3 .1}(t), \int_{9.4 .1}(t)\right\}$ elements.

If there is some $x \in X^{\prime}$ that blocks at least $\sqrt{9.1 .3}(t)$ displayed 3-separations of $M$. then by Theorem 9.1.3 there is a minor of $M$ that is isomorphic to a rank- $t$ circular ladder. If the crossing graph of $X^{\prime}$ is a star, then, since $n \geq \sqrt{9.2 .1}(t)$, by Theorem 9.2.1 $M$ has a rank- $t$ spike, a rank- $t$ double wheel, a rank- $t$ non graphic double wheel or $M^{*}\left(K_{3, t}\right)$ as a minor. The remainder of the proof is similar and left to the reader. 


\section{Chapter 10}

\section{Summing Up and Future Work}

The two main theorems of this thesis are the following.

Theorem. There is a function $f$ such that if $M$ is a 4-connected matroid of rank $f(n)$ with an $M\left(K_{3, f(n)}\right)$ or $M^{*}\left(K_{3, f(n)}\right)$ minor and no minor that that is $M^{*}\left(K_{3, t}\right)$ blocked in a path-like way, then $M$ must have a minor isomorphic to one of

1. $N\left(K_{3, n}\right)$,

2. $M\left(K_{4, n}\right)$,

3. $\left(N\left(K_{3, n}\right)\right)^{*}$,

4. $M^{*}\left(K_{4, n}\right)$,

5. an n-rung circular ladder,

6. an n-rung Möbius ladder,

7. a rank-n a double wheel,

8. a rank-n non graphic double wheel.

Theorem. There is a function $f$ such that the following holds. If $M$ is a binary matroid with coindependent set $X$ such that $M \backslash X$ has a swirl-like pseudo-flower of order $n$ and every 3-separation of $M \backslash X$ displayed by $F$ is blocked by an element of $X$, then $M$ has a minor isomorphic to one of the following.

1. $N\left(K_{3, n}\right)$, 
2. $M\left(K_{4, n}\right)$,

3. $\left(N\left(K_{3, n}\right)\right)^{*}$,

4. $M^{*}\left(K_{4, n}\right)$,

5. an n-rung circular ladder,

6. an n-rung Möbius ladder,

7. a rank-n a double wheel,

8. a rank-n non graphic double wheel,

9. a rank-n spike,

10. $M^{*}\left(K_{3, t}\right)$ blocked in a path-like way

11. a rank-n clam.

The aim of this thesis was to find the unavoidable minors of binary 4-connected matroids which unfortunately we have not been able to do in the given time. The next step on the way to this result will be to prove the following conjecture.

Conjecture 10.0.1. For every $n$ there is an $m$ such that the following holds. Let $M$ is binary 4-connected matroid with a minor $N$ that has a swirl-like pseudo-flower of order $m$ such that all 3-separations displayed by $F$ are bridged in $M$. Then either $M$ or $M^{*}$ has a minor $M^{\prime}$ such that the following hold.

i) $M^{\prime}$ has a spanning restriction $N^{\prime}$,

ii) $N^{\prime}$ has a swirl-like pseudo-flower $F^{\prime}$, and

iii) all 3-separations of $N^{\prime}$ displayed by $F^{\prime}$ are blocked in $M^{\prime}$.

We believe that we know how to prove this so hopefully this will be an easy job. Once we have proved Conjecture 10.0.1 the problem of finding the unavoidable minors of binary 4-connected matroids with a wheel minor and no large spike minor will have been resolved up to an analysis of clams.

The first step now is to completely resolve the case when we are blocking $M^{*}\left(K_{3, t}\right)$ in a path-like way. 
The next step will be the find the unavoidable minors of binary 4-connected matroids with a large spike minor. We expect this to be no more difficult than the analysis of binary 4-connected matroids with an $M\left(K_{3, n}\right)$-minor. We believe that all the techniques needed for this will be techniques used in this thesis, although judging by previous results we may be a little overoptimistic in this belief.

Once we have found the unavoidable minors of a large binary 4-connected matroid with a rank $n$-spike, $M\left(K_{3, n}\right), M^{*}\left(K_{3, n}\right)$ or rank- $n$ wheel minor we will have a complete set of unavoidable minors for the set of binary 4-connected matroids with no clam minor.

The final step to complete the analysis of unavoidable minors of large binary 4connected matroids is an analysis of the clam outcome. Unfortunately we do not yet have a good idea of how to do this or how long this analysis will take.

We also plan to continue with this project and attempt to find unavoidable minors of representable 4-connected matroids and unavoidable minors of general 4-connected matroids. Of course we really want to be able to be able to dispense with the assumption of 4-connectedness and find unavoidable minors of matroids containing a large highly connected component. This should take approximately until I die and possibly well beyond. 


\section{Bibliography}

[1] J. Aikin and J. Oxley. The structure of crossing separations in matroids. Advances in Applied Mathematics, 41(1):10 - 26, 2008.

[2] Robert E. Bixby. A simple theorem on 3-connectivity. 1982.

[3] N. Brettell, G. Whittle, and A. Williams. N-detachable pairs in 3-connected matroids i: unveiling x. submitted, 042018.

[4] D. Chun and J. Oxley. Capturing two elements in unavoidable minors of 3-connected binary matroids. Advances in Applied Mathematics, 50(1):155 $-175,2013$. Special issue in honor of Geoff Whittle on his 60th birthday.

[5] W. Cunningham and J. Edmonds. A combinatorial decomposition theory. Canadian Journal of Mathematics, 32(3):734765, 1980.

[6] R. Diestel. Graph Theory (Graduate Texts in Mathematics). Springer, August 2005 .

[7] G. Ding, B. Oporowski, J. Oxley, and D. Vertigan. Unavoidable minors of large 3-connected binary matroids. Journal of Combinatorial Theory, Series $B, 66(2): 334-360,1996$.

[8] G. Ding, B. Oporowski, J. Oxley, and D. Vertigan. Unavoidable minors of large 3-connected matroids. Journal of Combinatorial Theory, Series B, 71(2):244293, 1997.

[9] J. Geelen and B. Joeris. A generalization of the grid theorem. submitted, 09 2016.

[10] J. Geelen and G. Whittle. Inequivalent representations of matroids over prime fields. Advances in Applied Mathematics, 51(1):1 - 175, 2013. 
[11] K. Kunen. Set Theory. Studies in logic. College Publications, 2011.

[12] B. Oporowski, J. Oxley, and R. Thomas. Typical subgraphs of 3- and 4connected graphs. Journal of Combinatorial Theory, Series B, 57(2):239 257, 1993.

[13] J. Oxley. Matroid Theory (Oxford Graduate Texts in Mathematics). Oxford University Press, Inc., New York, NY, USA, 2006.

[14] J. Oxley, C. Semple, and G. Whittle. The structure of the 3-separations of 3connected matroids. Journal of Combinatorial Theory, Series B, 92(2):257 - 293, 2004. Special Issue Dedicated to Professor W.T. Tutte.

[15] James G. Oxley. On nonbinary 3-connected matroids. Transactions of the American Mathematical Society, 300(2):663-679, 1987.

[16] A. Shantanam. Unavoidable minors of large 5-connected graphs. Thesis, 2016.

[17] W. T. Tutte. Connectivity in matroids. Canadian Journal of Mathematics, 18:13011324, 1966. 